
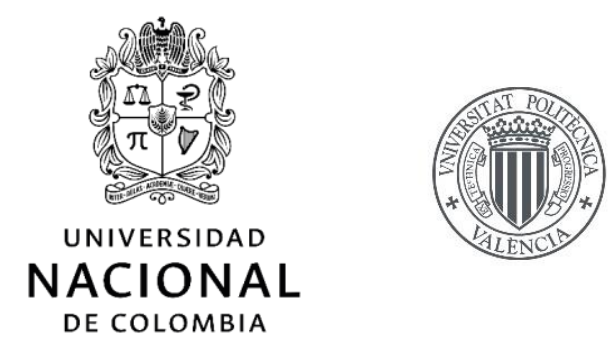

\title{
THE EFFECT OF ELECTRIC FIELDS ON \\ HYALINE CARTILAGE: AN IN VITRO \\ AND IN SILICO STUDY
}

\author{
Ph.D. Thesis by \\ Juan Jairo Vaca González \\ Thesis supervisors: \\ Ph.D., Gloria Gallego Ferrer \\ Ph.D., Diego Alexander Garzón Alvarado \\ Thesis co-advisor: \\ Ph.D., Johana María Guevara Morales
}

\author{
Universidad Nacional de Colombia \\ Department of Mechanical and Mechatronics Engineering \\ Universitat Politècnica de València \\ Centre for Biomaterials and Tissue Engineering \\ February - 2019
}





\title{
THE EFFECT OF ELECTRIC FIELDS ON \\ HYALINE CARTILAGE: AN IN VITRO \\ AND IN SILICO STUDY
}

\author{
Juan Jairo Vaca González
}

Co-tutelle Ph.D. Thesis to obtain the degree of Doctor in

Engineering - Science and Technology of Materials

Universidad Nacional de Colombia

Bogotá (Colombia)

and

Technologies for Health and Well-Being

Universitat Politècnica de València

Valencia (Spain) 

I dedicate this goal to,

My mother, a brave woman who taught me that everything you learn should be used to improve the welfare of humanity, especially, the health sciences.

My siblings, wonderful and brilliant people who taught me to be disciplined to achieve my goals. From you I acquired the sense of responsibility to apply science and enhance the future of our society.

To all my relatives, especially my academic father, Professor Carlos Caicedo, who has guided and accompanied me through my academic training. Thanks to your advice, today the bioengineering is my future.

To Claudia Calderón, for the love she gives me every day. Your pleasant company and words of support made this academic process a great memory for life. 



\section{Acknowledgements}

I want to thank all the people who were by my side supporting and giving me advice during these four years. Thanks to you I was able to make from this doctorate the most incredible and unforgettable experience in my life.

To the financial support from COLCIENCIAS - COLFUTURO through the fellowship No. 647 for national doctorates. To the financial support from COLCIENCIAS through the research grant 712-2015 No. 50457. To the financial support from the Spanish Ministry of Economy and Competitiveness through the MAT2016-76039-C4-1-R project.

To my thesis advisors, Dr. Diego Alexander Garzón Alvarado and Dr. Gloria Gallego Ferrer for teaching, guiding and helping me during my doctoral degree. Your advice strengthened my research skills and made that tissue engineering became my passion.

To my co-advisor, colleague and friend Dr. Johana Guevara. Thank you for giving me your friendship and for helping me to grow as a researcher and person.

To Dr. Yoshie Hata for sharing her time and knowledge to carry out the experimental procedures in the Biomimetics Laboratory at Universidad Nacional of Colombia.

To Dr. Sandra Shefelbine for sharing her knowledge and experience and for inviting me to have a great experience in her Biomechanics Laboratory at Northeastern University.

To my friends and colleagues Sandra Clara, María Guillot and Ximo for sharing their time and knowledge to carry out the experimental procedures in the culture laboratory of the Centre for Biomaterials and Tissue Engineering at Universitat Politècnica of València. 
To Dr. José Luis Gómez Ribelles for his valuable help and brilliant ideas.

To professor Maria Jesús Sanchís Sánchez from Thermodynamics Department at Universitat Politècnica of València for helping me with the dielectric characterization of the hydrogel and culture media.

To my colleague and friend Gustavo Navarro and Valteam Research \& Innovation for helping me to improve the design of the biophysical stimulator.

To my friends from the Group of Modeling and Numerical Methods in Engineering and Group of Mechanobiology in Tissues and Organs at the Biotechnology Institute of the Universidad Nacional of Colombia, who shared their knowledge to make this possible.

To my friends from the Centre for Biomaterials and Tissue Engineering at Universitat Politècnica of València, who gave me their friendship and made my time in Spain one of my best memories. 


\section{Table of contents}

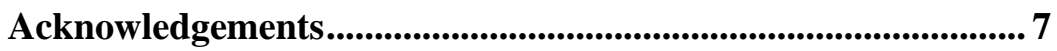

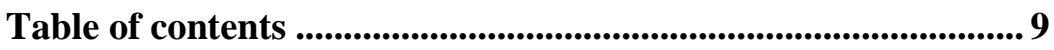

List of figures ..................................................................... 16

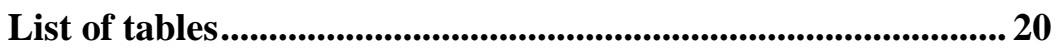

List of appendices...................................................................................... 21

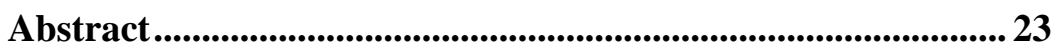

Resumen ........................................................................................................ 24

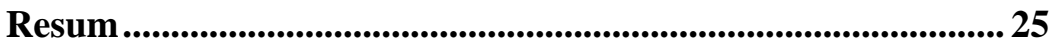

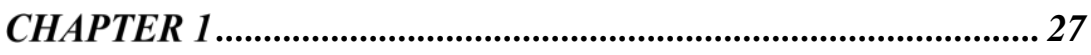

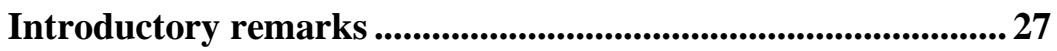

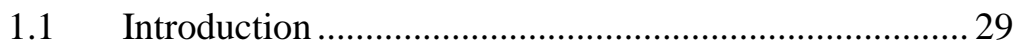

$1.2 \quad$ Hypothesis and objectives ................................................ 31

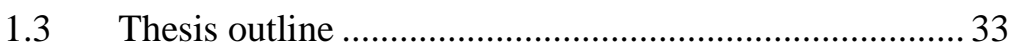

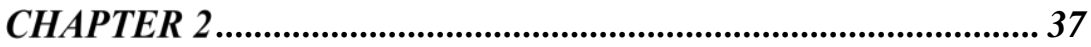

Hyaline cartilage: structure, pathologies and therapeutic

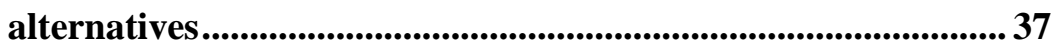

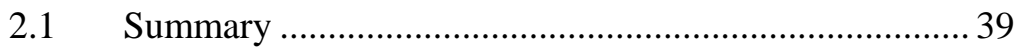

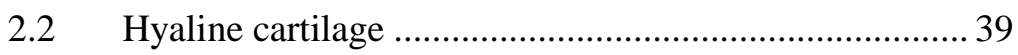

2.2.1 Embryological development of the articular cartilage

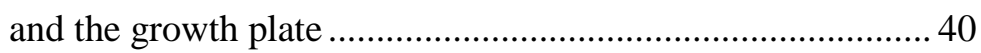

2.2.2 Anatomy of cartilaginous structures in long bones 41

2.2.2.1 Articular cartilage ................................................. 41

2.2.2.2 Growth plate ................................................... 44

2.2.3 Molecular components of the articular cartilage and the growth plate ...................................................................... 45

2.2.3.1 Articular cartilage .................................................. 45 


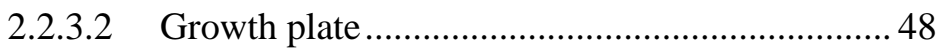

2.2.4 Biomechanical properties of hyaline cartilage ........50

2.3 Pathologies affecting hyaline cartilage............................ 52

2.3.1 Articular cartilage ..................................................... 53

2.3.2 Growth plate ........................................................... 53

2.4 Treatments to restore hyaline cartilage ……................... 54

2.4.1 Noninvasive treatments ........................................... 54

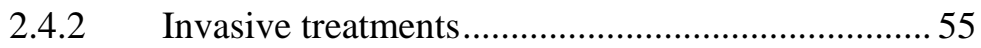

2.5 Hydrogels used for hyaline cartilage regeneration .......... 56

2.5.1 Hyaluronic acid - gelatin hydrogels......................... 58

2.5.1.1 Hyaluronic acid ................................................ 58

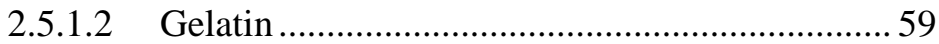

2.5.1.3 Enzymatic cross-linking of hyaluronic acid gelatin 60

2.5.1.4 Advantages of hyaluronic acid - gelatin hydrogels 62

2.5.1.5 Effect of hyaluronic acid - gelatin hydrogels in

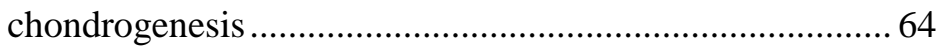

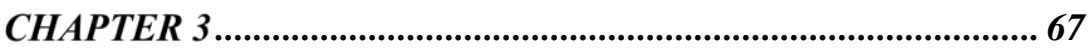

Mechanobiological modeling of endochondral ossification ...... 67

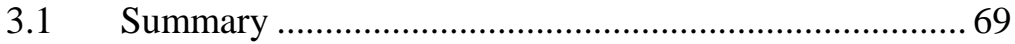

3.2 Introduction .................................................................. 70

3.3 Materials and methods ................................................... 73

3.3.1 Experimental data................................................... 73

3.3.1.1 Tissue samples................................................. 73

3.3.1.2 Measurement of length and thickness ................ 73

3.3.1.3 Alcian blue/Alizarin red staining ........................ 74

3.3.1.4 Histological and image analysis ......................... 74 


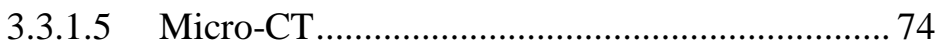

3.3.2 Mathematical model................................................. 75

3.3.2.1 Geometrical model and boundary conditions..... 75

3.3.2.2 Mechanical factors and material properties........ 76

3.3.2.3 Molecular regulatory system ............................... 77

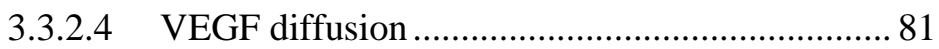

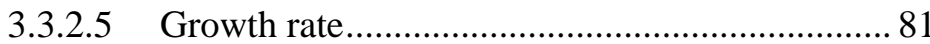

3.3.2.6 Bone collar formation........................................ 83

3.3.2.7 Bone elongation and ossification ........................ 83

3.3.2.8 Model Implementation ......................................... 84

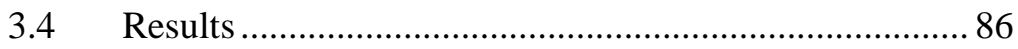

3.4.1 Femur Morphogenesis................................................ 86

3.4.2 Biochemical Regulation ......................................... 88

3.4.3 Hydrostatic Stress and Fluid Velocity Distribution 90

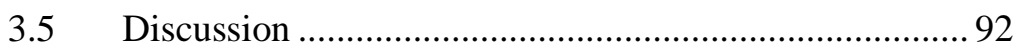

3.6 Concluding Remarks ....................................................... 94

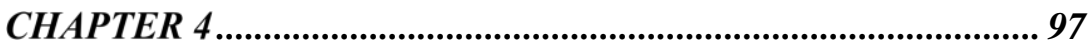

Biophysical stimuli: a review of electrical and mechanical stimulation in hyaline cartilage ........................................................... 97

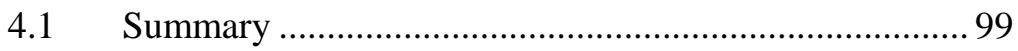

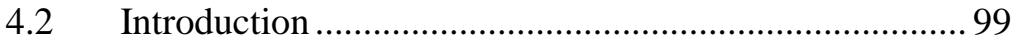

4.3 Electrical stimulation for tissue engineering hyaline

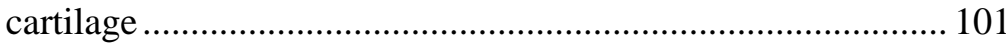

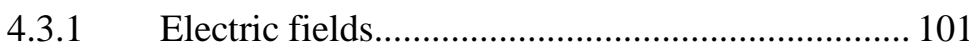

4.3.1.1 In vivo studies ................................................ 101

4.3.1.2 In vitro studies.............................................. 102

4.3.1.3 Studies performed in explants and 3-Dimensional cartilage constructs ............................................................ 105 
4.3.1.4 Effect of EFs over the growth plate.................. 105

4.3.2 Electromagnetic fields ........................................... 105

4.3.2.1 In vivo use of EMFs to heal osteoarthritis........ 107

4.3.2.2 In vitro studies using cell cultures ..................... 107

4.3.2.3 Studies performed in explants 3-Dimensional cartilage constructs ............................................................... 107

4.3.2.4 Effect of the EMFs over the growth plate ........ 108

4.4 Mechanical Stimulation for Tissue Engineering Hyaline

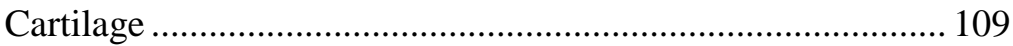

4.4.1 Compressive loads................................................. 109

4.4.1.1 In vivo studies ................................................. 109

4.4.1.2 In vitro studies: tissue cultures ......................... 112

4.4.1.3 In vitro studies: cell cultures ............................ 112

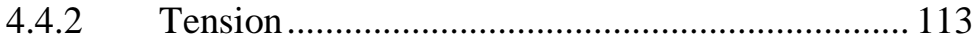

4.4.3 Hydrostatic pressure ............................................ 113

4.4.3.1 In vitro studies: tissue cultures .......................... 113

4.4.3.2 Studies performed in cartilage explants and 3-

Dimensional constructs ..................................................... 114

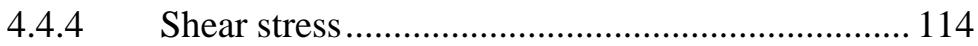

4.5 Cellular mechanisms involved in transduction of

biophysical stimuli ................................................................... 116

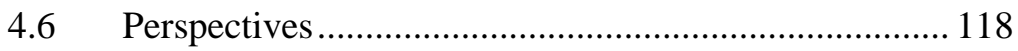

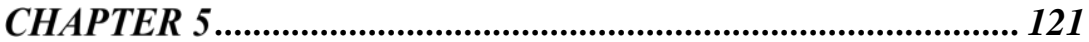

An in vitro chondrocyte electrical stimulation framework ..... 121

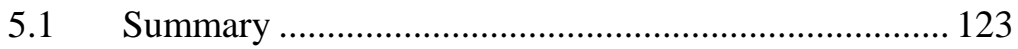

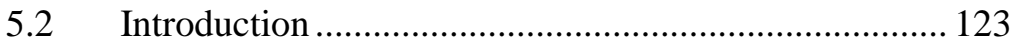

5.3 Materials and methods ................................................ 125

5.3.1 Chondrocyte isolation and cell culture ................. 125 
5.3.2 Chondrocyte culture medium characterization and EF estimation

5.3.2.1 Capacitance and permittivity of the cell culture medium $(\mathrm{Ccm}, \mathrm{\varepsilon cm})$

5.3.2.2 Complex permittivity and conductivity of the cell culture medium $(\varepsilon j c m, \sigma c m)$ 128

5.3.2.3 Estimation of EFs 129

5.3.3 Electrical stimulation device 130

5.3.4 Electrical stimulation assay...... 131

5.3.5 Proliferation and cell death assay 131

5.3.6 Glycosaminoglycan quantification. 131

5.3.7 Statistical analysis 132

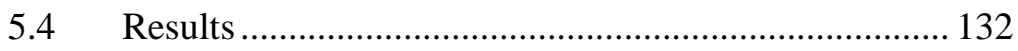

5.4.1 Cell culture medium characterization and EF estimation

5.4.2 Proliferation and cell death..................................... 134

5.4.3 Glycosaminoglycans synthesis............................. 134

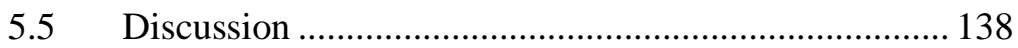

5.6 Concluding Remarks .................................................. 142

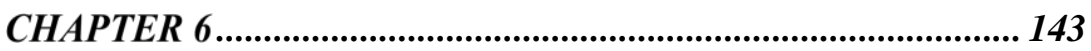

Capacitively coupled electrical stimulation of rat

chondroepiphysis explants............................................................. 143

6.1 Summary .................................................................. 145

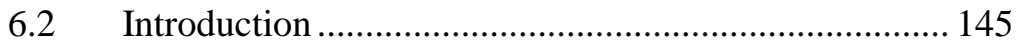

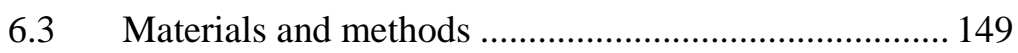

6.3.1 Chondroepiphysis isolation and in vitro culture... 149

6.3.2 Electric field estimation ........................................ 149

6.3.3 Electrical stimulation assay …………………....... 150 
6.3.4 Growth plate histological analysis ....................... 153

6.3.4.1 Masson's trichrome staining ….......................... 153

6.3.4.2 Quantitative analysis of growth plate zones..... 153

6.3.5 Statistical analysis ................................................ 154

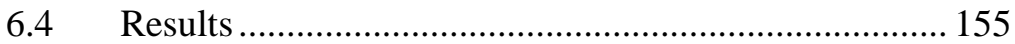

6.4.1 Estimation of EFs ............................................... 155

6.4.2 Histomorphometric of the growth plate ............... 157

6.4.2.1 Thickness and morphology of growth plate zones 157

6.4.2.2 Columnar analysis of growth plate zones......... 160

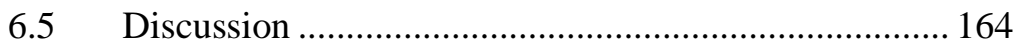

6.6 Concluding Remarks ................................................. 166

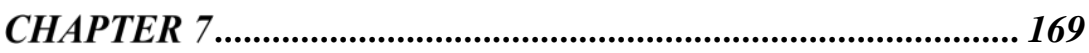

Electrical stimulation enhances chondrogenic differentiation of mesenchymal stem cells cultured in hyaluronic acid - gelatin hydrogels ..................................................................................................... 169

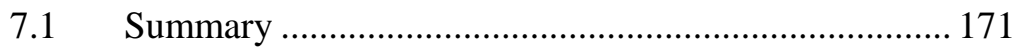

7.2 Introduction ............................................................. 172

7.3 Materials and methods ................................................... 174

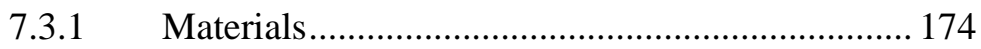

7.3.2 Synthesis of HA-GEL hydrogels.......................... 176

7.3.3 Tyramine substitution degree in HA and GEL..... 177

7.3.4 Hydrogels preparation ............................................ 177

7.3.5 Rheological measurements of HA-GEL hydrogel178

7.3.6 Dielectric constants of HA-GEL hydrogels and

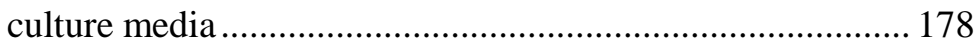

7.3.7 Estimation of EFs ............................................... 179

7.3.8 Mesenchymal stem cell isolation and cell culture 180 
7.3.9 Electrical stimulation assay ..................................... 181

7.3.10 Morphology and cell proliferation assay ............... 181

7.3.11 Assessment of chondrogenic markers .................. 182

7.3.12 Gene expression assay ......................................... 183

7.3.13 Statistical analysis ................................................... 184

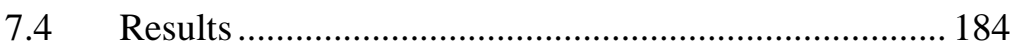

7.4.1 HA molecular weight ........................................... 184

7.4.2 Tyramine substitution degree in HA and GEL..... 185

7.4.3 Mechanical properties of the hydrogels ............... 186

7.4.4 Electrical properties of the hydrogel .................... 188

7.4.5 Distribution of EFs ............................................... 190

7.4.6 Morphology and cell proliferation ........................ 191

7.4.7 Chondrogenic differentiation ................................ 193

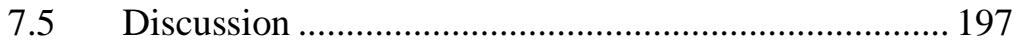

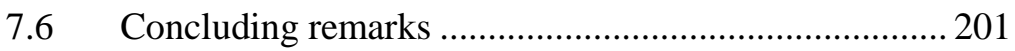

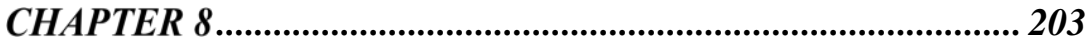

Conclusions and perspectives............................................................ 203

Bibliography ........................................................................................ 209

Appendices .................................................................................................. 241 


\section{List of figures}

Figure 2-1: Endochondral ossification process. ................................. 41

Figure 2-2: Mechanical loads supported by articular cartilage. ......... 42

Figure 2-3: Scheme of the articular cartilage zones........................... 43

Figure 2-4: Scheme of the growth plate zones.................................... 44

Figure 2-5: Representative scheme of PGs, collagen fibers and GAGs.

Figure 2-6: Schematic representation of molecular regulations in the growth plate. 48

Figure 2-7: Chemical structure of hyaluronic acid. It is constituted by a repetition of units of D-glucuronic acid and $\mathrm{N}$-acetyl glucosamine... 58 Figure 2-8: Chemical structure of gelatin. It is constituted by a repetition of units such as glycine, proline and hydroxyproline.

Figure 2-9: Reaction of hyaluronic acid or gelatin chains to form EDC carbocation (1) and O-acylisourea (2) 60 Figure 2-10: Representative scheme of the chemical reaction for obtaining hyaluronic acid or gelatin grafted with tyramine using $\mathrm{O}$ acylisourea. 61

Figure 2-11: Diagram of the cross-link bonds formed in the gelation of hyaluronic acid and gelatin hydrogels using $\mathrm{HRP}$ and $\mathrm{H}_{2} \mathrm{O}_{2}$. 62

Figure 3-1: Generic long bone parts.................................................. 72

Figure 3-2: Representative scheme of the anlage geometry and boundary conditions. 75

Figure 3-3. Representative scheme of bone elongation and ossification on the growth plate zone. 84 
Figure 3-4: Representative scheme of the multiscale computational modeling 85

Figure 3-5. Length and thickness curves of a Balb/c mice femur $(\mathrm{n}=3 /$ time point $)$.

Figure 3-6. Comparison between the computational model and the experimental data. 88

Figure 3-7. Bone morphology with and without mechanical load. .... 89 Figure 3-8. Representative scheme of molecular concentrations at E19.

Figure 3-9. Hydrostatic stress and fluid velocity distributions within the cartilage at E19. 91

Figure 4-1: Schematic diagram of an indirect coupling system to stimulate in vitro cell cultures. 102 Figure 4-2: Schematic diagram of the devices used to apply mechanical loads to chondrocytes cultured in 3 dimensional (3D) structures. Both schemes have in common a loading cell, a cell culture well plate and the $3 \mathrm{D}$ construct. 111

Figure 5-1: Representative scheme of a parallel plate capacitor to determinate the EFs.

Figure 5-2: Schematic diagram for electrode position and electronic circuit for cell cultures stimulations. 130

Figure 5-3: Distribution and estimation of the EFs at different heights ( $\mathrm{Z}$ coordinate) of the capacitive system.

Figure 5-4: Cell population of stimulated and non-stimulated cell cultures. 135

Figure 5-5: Cell death bar graphs for stimulated cell cultures and controls 136 
Figure 5-6: Production of GAGs per cell for stimulated cell cultures and controls

Figure 6-1: Capacitively coupling electrical system. 150

Figure 6-2. Schematic diagram of the in vitro stimulation 152

Figure 6-3. Representative diagram for the quantitative analysis of growth plate zones 154

Figure 6-4. Representative scheme of the EFs in the capacitively coupling system generated by a voltage $100 \mathrm{Vpp}$ at $60 \mathrm{kHz}$ sine waveform. 156

Figure 6-5. EF intensities within the capacitively coupled system applying a voltage $100 \mathrm{Vpp}$ at $60 \mathrm{kHz}$ sine wave-form.

Figure 6-6. Measurement of growth plate lengths and thickness of P-z, $\mathrm{PH}-\mathrm{z}$ and Ht-Z. 158

Figure 6-7. Histologies of growth plates at different times of culture. 159

Figure 6-8. Measurement of $\boldsymbol{C} \boldsymbol{i}$ and $\boldsymbol{C} \boldsymbol{c}$ in growth plate zones of femur explants. 161

Figure 6-9. Measurement of $\boldsymbol{C D}$ and inclination angle $\alpha$ of columns in growth plate zones of femur explants. 162

Figure 6-10. Representative scheme of histologies showing a hypertrophic cell condensation in the center of the chondroepiphysis of femurs and humerus. 163

Figure 7-1: Molecular weight of HA 185

Figure 7-2: Gelation dynamics and strain amplitudes of hydrogels. 187 Figure 7-3: Dynamic frequency sweep test to determine the dependence of the dynamic shear modulus and loss factor on the frequency...... 188 Figure 7-4: Dielectric properties of the hydrogels and culture medium. 
Figure 7-5: Distribution of the EFs within the cell culture. 190 Figure 7-6: DAPI/actin staining to control and stimulated hydrogels at days 14 and 21 of culture. Scale bar $=50 \mu \mathrm{m}$. 191 Figure 7-7: Phase contrast images of control and stimulated hydrogels at days 2, 7, 14 and 21 of culture. Scale bar $=50 \mu \mathrm{m}$. 192 Figure 7-8: Cell proliferation of controls and stimulated hydrogels. 193 Figure 7-9: Immunofluorescence images for SOX-9 and Aggrecan of MSCs cultured in HA-GEL hydrogels for 14 days. Scale bar $=50 \mu \mathrm{m}$.

Figure 7-10: Immunofluorescence images for SOX-9 and Aggrecan of MSCs cultured in HA-GEL hydrogels for 21 days. Scale bar $=50 \mu \mathrm{m}$. 195

Figure 7-11: Quantification of chondrogenic markers of MSCs encapsulated in the HA-GEL hydrogels. 196 


\section{List of tables}

Table 3-1: Material properties used in finite element model. 77

Table 4-1: Summary of EFs application to in vivo and in vitro systems. 103

Table 4-2: Summary of EMFs application to in vivo, vitro, cartilage explants and scaffolds. 106

Table 4-3: Summary of some representative studies using compressive loads on hyaline cartilage. 110

Table 4-4: Summary of some representative studies using hydrostatic pressure on hyaline cartilage.

Table 5-1: The $\boldsymbol{C} \boldsymbol{m} \boldsymbol{c}$ and $\boldsymbol{\varepsilon} \boldsymbol{m} \boldsymbol{c}$ at $60 \mathrm{kHz}$ sine wave-form. 132 Table 5-2: Electrical constants in vacuum (Plexiglas) and with cell culture medium ( $\boldsymbol{\sigma m} \boldsymbol{c}$ and $\boldsymbol{\varepsilon j m c})$

Table 5-3: Summary of biological effects of EFs applied to cell cultures.

Table 6-1. Dielectric properties and measurements used in the simulation. 151

Table 7-1: Dielectric properties and measurements used in the simulation. 179

Table 7-2: Forward (F) and Reverse (R) primers for pig targets. .... 184 Table 7-3: Graft tyramine concentration values and substitution degree in the synthesized products. 186 


\section{List of appendices}

Appendix A: Value of the parameters used in the simulations herein described. 241

Appendix B: Endochondral ossification process for all cases studied in the computational model. 243 Appendix C: Variation of the length and the thickness of the POC and distal and proximal epiphysis when the mechanical load was applied with different frequencies and signals

Appendix D: Molecular diffusion during bone growth. 246 Appendix E: Bone morphology and Ihh, PTHrP and VEGF diffusion for simulations performed with high magnitude mechanical loading (1, 10 and $100 \mathrm{MPa}$ ). 249 Appendix F: Representative scheme of the EFs in the capacitively coupling system generated by a voltage $50 \mathrm{Vpp}$ at $60 \mathrm{kHz}$ sine waveform. 250

Appendix G: EF intensities within the capacitive coupled system applying a voltage $50 \mathrm{Vpp}$ at $60 \mathrm{kHz}$ sine wave-form. 251

Appendix H: Measurement of growth plate lengths and thickness of P$\mathrm{z}, \mathrm{PH}-\mathrm{z}$ and Ht-z. 252 Appendix I: Measurement of $\boldsymbol{C} \boldsymbol{i}$ and $\boldsymbol{C} \boldsymbol{c}$ in growth plate zones of humerus explants. 253 Appendix J: Measurement of $\boldsymbol{C D}$ and inclination angle $\alpha$ of columns in growth plate zones of humerus explants. 254 



\section{Abstract}

Hyaline cartilage is a dense connective tissue with low self-healing capacity when is affected by degenerative pathologies. Therefore, electrical stimulation has been proposed as a possible non-invasive alternative therapy to enhance the restoration of the cartilaginous tissue. Accordingly, this work presents a combined computational and experimental approach to understand better the hyaline cartilage biology and its response to electrical stimulation using different in vitro models. On the one hand, a mechanobiological model was developed to simulate the endochondral ossification process. On the other hand, the electrical stimulation on hyaline cartilage was evaluated in three different scenarios. Initially, cell proliferation and glycosaminoglycans synthesis of chondrocytes, cultured in monolayer and stimulated with electric fields, was analyzed. Then, a histomorphometric analysis was performed to chondroepiphysis explants that were electrically stimulated. Finally, the effects of the electric fields on chondrogenic differentiation of mesenchymal stem cells cultured in hydrogels was assessed. The results indicated that electrical stimulation is a promising biophysical stimulus, due to the fact that this type of stimulation enhances the viability and the proliferation of cells, induces morphological changes in the chondrocytes, and stimulates the synthesis of the main molecules that compose the hyaline cartilage, such as SOX-9, glycosaminoglycans and aggrecan. Moreover, this project is the first step towards the implementation of an alternative biophysical stimulus that modifies the cellular dynamics of growth plate chondrocytes in ex vivo conditions. Additionally, this study highlights the potential effect of electric fields to induce the chondrogenesis process of mesenchymal stem cells cultured in basal conditions. Overall, the assessment of electrical stimulation on chondrocytes, tissues and scaffolds is a useful tool that may contribute to the current knowledge of regenerative therapies focused on hyaline cartilage healing. 


\section{Resumen}

El cartílago hialino es un tejido conectivo denso con poca capacidad de auto regeneración cuando es afectado por patologías degenerativas. Por lo tanto, la estimulación eléctrica se ha propuesto como una terapia alternativa no invasiva para mejorar la reparación del cartílago hialino. De acuerdo con esto, este trabajo presenta un enfoque computacional y experimental combinado para entender mejor la biología del cartílago hialino y su respuesta a la estimulación eléctrica usando diferentes modelos in vitro. En primer lugar, se ha desarrollado un modelo mecanobiológico para simular el proceso de osificación endocondral. Por otro lado, se ha evaluado el efecto de la estimulación eléctrica sobre el cartílago hialino en tres escenarios diferentes. Inicialmente se ha analizado la proliferación celular y la síntesis de glicosaminoglicanos de condrocitos cultivados en monocapa y estimulados con campos eléctricos. Luego, se ha realizado un análisis histomorfométrico a explantes de condroepífisis que fueron estimulados eléctricamente. Por último, se ha evaluado el efecto de los campos eléctricos sobre la diferenciación condrogénica de células madre mesenquimales cultivadas en hidrogeles. Los resultados indican que la estimulación eléctrica es un estímulo biofísico prometedor, ya que este tipo de estimulación mejora la viabilidad y la proliferación celular, induce cambios morfológicos en los condrocitos, y estimula la síntesis de las principales moléculas que componen el cartílago hialino, tales como SOX-9, glicosaminoglicanos y agrecan. Además, este proyecto es el primer paso hacia la implementación de un estímulo biofísico alternativo que modifica la dinámica celular de los condrocitos de la placa de crecimiento en condiciones ex vivo. Adicionalmente, este estudio resalta el efecto potencial de los campos eléctricos para inducir el proceso de condrogénesis de células madre mesenquimales cultivadas en condiciones basales. En general, la evaluación de la estimulación eléctrica sobre condrocitos, tejidos y andamios es una herramienta útil que puede contribuir al conocimiento actual de las terapias regenerativas enfocadas en la regeneración del cartílago hialino. 


\section{Resum}

El cartílag hialí és un teixit connectiu dens amb poca capacitat d'auto regeneració quan es veu afectat per patologies degeneratives. Per tant, l'estimulació elèctrica s'ha proposat com una teràpia alternativa no invasiva per millorar la reparació del cartílag articular. D'acord amb això, aquest treball presenta un enfoc computacional i experimental combinat per entendre millor la biologia del cartílag hialí i la seva resposta a l'estimulació elèctrica usant diferents models in vitro. En primer lloc, s'ha desenvolupat un model mecanobiològic per simular el procés d'ossificació endocondral. D'altra banda, s'ha avaluat l'efecte de l'estimulació elèctrica sobre el cartílag hialí en tres escenaris diferents. Inicialment s'ha analitzat la proliferació cel-lular i la síntesi de glicosaminoglicans de condròcits cultivats en monocapa i estimulats amb camps elèctrics. Després, s'ha realitzat una anàlisi histomorfomètrica a explants de condroepífisis que van ser estimulats elèctricament. Finalment, s'ha avaluat l'efecte dels camps elèctrics sobre la diferenciació condrogénica de cèl-lules mare mesenquimals cultivades en hidrogels. Els resultats indiquen que l'estimulació elèctrica és un estímul biofîsic prometedor, ja que aquest tipus d'estimulació millora la viabilitat i la proliferació cel-lular, indueix canvis morfològics en els condròcits, i estimula la síntesi de les principals molècules que componen el cartílag hialí, com ara SOX-9, glicosaminoglicans i agrecan. A més, aquest projecte és el primer pas cap a la implementació d'un estímul biofísic alternatiu que modifica la dinàmica cel-lular dels condròcits de la placa de creixement en condicions ex vivo. Addicionalment, aquest estudi ressalta l'efecte potencial dels camps elèctrics per induir el procés de condrogènesi de cèl-lules mare mesenquimals cultivades en condicions basals. En general, l'avaluació de l'estimulació elèctrica sobre condròcits, teixits i scaffolds és una eina útil que pot contribuir al coneixement actual de les teràpies regeneratives enfocades a la regeneració del cartílag hialí. 



\section{CHAPTER 1}

\section{Introductory remarks}





\subsection{Introduction}

Hyaline cartilage is an avascular tissue formed by a single cell type, the chondrocyte. This cell is responsible of synthetizing the two major loadbearing macromolecules located in cartilaginous tissue: Collagens and Proteoglycans. The hyaline cartilage, in the skeletal system, is found in two specialized structures of the long bones: the articular cartilage and the growth plate. The former acts as a smooth, lubricated and low friction surface that receives mechanical loads and facilitates movement between the joints, while the latter is responsible for the longitudinal growth and shape of long bones. Considering that hyaline cartilage is exposed to a combination of mechanical loads, its avascularity and low proliferative capacity are counterproductive factors that limit the tissue self-healing process. It has been shown that mechanical loads affect the cell deformation, the fluid flow, the concentration of nutrients and ionic gradients, and the anabolic and catabolic activity of the cartilage extracellular matrix components. Accordingly, mechanical factors induce changes in the tissue altering both its morphology and its molecular behavior. Additionally, mechanical loads are a risk factor for the development of osteoarthritis, one of the main pathologies that affects the cartilaginous tissue. Taking this into account, several strategies have been developed for cartilage treatment such as surgical interventions or non-invasive treatments. Unfortunately, these treatments have short-term effect in cartilage repair; for instance, surgical interventions generate bad quality tissue (fibrocartilage). For this reason, novel therapeutic options are being implemented by tissue engineering in order to improve cartilage repair.

Considering that several biophysical and biochemical stimuli govern the development, maintenance and functionality of hyaline cartilage, it is necessary to understand their effect on cartilage biology in order to use these stimuli to generate tools that improve the production in vitro of the characteristic chondrogenic markers that compose the cartilaginous tissue. In this context, tissue engineering has focused on the development of biomimetic tissues that replace the biological, structural and functional characteristics of cartilage. Thus, this kind of tissues are mainly composed by biomaterials able to resist and adapt to 
the mechanical environment to which the cartilage is subjected. Such biomimetic tissues are generated using a combination of cellular, biochemical and biophysical factors. One of the biophysical stimuli used nowadays are electric fields, since they have proven to influence different cellular functions such as morphology, elongation, gene expression, proliferation and cell migration. Based on this, the use of electrical stimulation has been proposed as a tool for improving the techniques carried out by tissue engineering and regenerative medicine for hyaline cartilage recovery. However, reports in the literature about electrical stimulation in hyaline cartilage and chondrocytes are limited; in addition, the results have been contradictory. Taking this into account, the aim of this study was to assess the effect of electric fields on hyaline cartilage tissue and analyze the potential implications of the electrical stimulation in the biological responses of chondrocytes by using a combined computational and experimental approach. Consequently, the response of the electrical stimulation was evaluated in three different scenarios: monolayer cultures of chondrocytes, hyaline cartilage explants, and hydrogels as 3D constructs.

First, regarding monolayer cultures, the electric fields were applied in primary chondrocytes extracted from rodents. In this scenario, the cell proliferation, cell death and proteoglycans synthesis were analyzed. Second, in relation to hyaline cartilage explants, the electrical stimulation was applied over chondroepiphysis explants of femurs and humerus of rodents. Here, a histomorphometric analysis of the epiphyseal plate and the growth plate zones was performed. Finally, the effect of electric fields in the chondrogenesis process was evaluated on mesenchymal stem cells cultured within hyaluronic acid - gelatin hydrogels. In this third scenario, the cell proliferation and molecular synthesis were analyzed. As a complement of the scenarios carried out in this doctoral thesis, a mechanobiology model was developed in order to observe how stress distribution patterns and molecular gradients regulate the long bone growth in prenatal stages.

Experimental results showed that electric fields generate different effects over hyaline cartilage. It was demonstrated that electric fields induced chondrocyte proliferation and glycosaminoglycan synthesis in 
a monolayer environment. On the other hand, it was proven that electrical stimulation has directly influenced the chondrocytes morphology within the growth plate. Besides these results, it was evidenced that electric fields favored the chondrogenic differentiation of mesenchymal stem cells increasing proteoglycan synthesis and stimulating the production of transcription factors involved in chondrogenesis.

This project constitutes the first attempt of application of electric fields to the three biological scenarios currently used to restore hyaline cartilage: monolayer cultures, ex vivo cultures and scaffolds. The findings derived from this work may help to understand how the electrical stimuli induce morphological changes in chondrocytes and mesenchymal stem cells. Moreover, these conclusions may also allow to verify the influence of electric fields on the molecular events taking place within both the hyaline cartilage and the 3D constructs. Overall, this study not only explains the role of electric fields in the chondrogenesis process, but also provides useful information of new therapeutic approaches for hyaline cartilage treatment.

\subsection{Hypothesis and objectives}

Considering that, hyaline cartilage responds to external biophysical stimuli, it has been demonstrated that the dynamic of this tissue is modified by the application of external electric fields. In vitro assays have evaluated the effect of electric fields in chondrogenesis and cell behavior within hyaline cartilage using different culture systems such as monolayer cultures, explants and 3D constructs. These studies have focused on the assessment of viability, proliferation, synthesis of extracellular matrix components, morphological changes of growth plate chondrocytes and the potential of mesenchymal stem cells to differentiate into a chondrocytic lineage. Nevertheless, there are many discrepancies in the obtained results, because there are limitations in the voltage required to generate the electric fields, the stimulation time, and the amount of days that cell cultures need to be under stimulation. In addition, the methodology to generate homogeneously the electric fields and calculate their intensities is still a largely unexplored field. In 
this context, we hypothesize that the design and construction of a bioreactor, based on Wien bridge oscillator circuit, may generate and distribute homogeneously the electric fields over the complete surface of different in vitro cultures. In addition, the implementation of a combined experimental and computational approach will allow predicting the electric fields intensities that are stimulating the cells and tissues. Therefore, the implementation of a well-stablished protocol will allow the analysis of different electric field intensities and stimulation times in order to better understand the impact of the electrical stimulation on cell dynamics during the process of chondrogenesis, endochondral ossification, and hyaline cartilage maintenance.

Taking into account the aforementioned, we formulate the following aims in order to validate the proposed hypothesis:

\section{- General aim}

To assess the effect of an external electrical stimulation on hyaline cartilage from three different scenarios: chondrocyte monolayer cultures, chondroepiphysis explants and chondrogenic hydrogels.

\section{- Specific aims}

1. To design and built a bioreactor that distributes and applies homogeneously different intensities of external electric fields to monolayer cultures, cartilage explants and 3D constructs.

2. To evaluate in vitro the effect of external electric fields in terms of viability, proliferation and molecular synthesis of chondrocytes cultured in monolayer.

3. To evaluate in vitro the effect of external electric fields in terms of morphophysiology of growth plate chondrocytes.

4. To evaluate in vitro the effect of external electric fields in terms of chondrogenic differentiation of mesenchymal stem cells cultured in an injectable hydrogel. 


\subsection{Thesis outline}

This thesis is presented by a compendium of six publications, where five articles are already published in scientific journals and one is in the process of being published. The articles and Reprints and Permissions obtained from the journals, to include the pre-print articles in this thesis, are attached at the end of the document. The articles are the following:

Article 1: J. J. Vaca-González, M. L. Gutiérrez, and D. A. GarzónAlvarado, "Cartílago articular: estructura, patologías y campos eléctricos como alternativa terapéutica. Revisión de conceptos actuales". Rev. Colomb. Ortop. y Traumatol., vol. 31, no. 4, pp. $202-$ 210, 2017.

Article 2: J. J. Vaca-González, M. Moncayo-Donoso, J. M. Guevara, Y. Hata, S. J. Shefelbine, and D. A. GarzónAlvarado, "Mechanobiological modeling of endochondral ossification: an experimental and computational analysis”. Biomech. Model. Mechanobiol., vol. 17, no. 3, pp. 853-875, 2018.

Article 3: J. J. Vaca-González, J. M. Guevara, M. A. Moncayo, H. Castro-Abril, Y. Hata, and D. A. Garzón-Alvarado, "Biophysical Stimuli: A Review of Electrical and Mechanical Stimulation in Hyaline Cartilage”. Cartilage, Sep. 2017.

Article 4: J.J. Vaca-González, J. Guevara, J. Vega, and D. GarzónAlvarado, "An In Vitro Chondrocyte Electrical Stimulation Framework: A Methodology to Calculate Electric Fields and Modulate Proliferation, Cell Death and Glycosaminoglycan Synthesis”. Cell. Mol. Bioeng., pp. 1-11, 2016.

Article 5: J.J. Vaca-González, J.F. Escobar, J.M. Guevara, Y.A. Hata, G. Gallego Ferrer and D.A. Garzón-Alvarado, "Capacitively Coupled Electrical Stimulation of Rat Chondroepiphysis Explants: A Histomorphometric Analysis". Bioelectrochemistry, 126, 1-11, 2019. 
Article 6: J.J. Vaca-González, S. Clara-Trujillo, M. Guillot-Ferriols, J. Ródenas-Rochina, M.J. Sanchis Sánchez, D.A. Garzón-Alvarado, J.L. Gómez Ribelles and G. Gallego Ferrer, "Coupled Capacitive Electric Fields Over Hyaluronic Acid - Gelatin Hydrogels Enhance Chondrogenic Differentiation". (In preparation).

The thesis conceptual framework, methodological approach, results, discussion and conclusions contained within this document are organized in 8 chapters. CHAPTER 1 corresponds to this introductory section. CHAPTER 2 presents a conceptual framework that summarizes the anatomical, molecular structure and biochemical properties of the hyaline cartilage. Additionally, descriptions regarding the pathological issues in articular cartilage and growth plate are introduced. To close this chapter, a brief description of some therapeutic alternatives is included, making special emphasis in hyaluronic acid - gelatin hydrogels to restore hyaline cartilage.

Considering that hyaline cartilage is presented in long bones, CHAPTER 3 introduces a mechanobiology model to simulate the formation of the cartilage epiphyses and the growth plates. This mathematical model simulated the anlage as a poroelastic material, while the biochemical regulation is simulated as a reaction-diffusion model. Additionally, an experimental approach to observe the bone development in prenatal stages was carried out in order to validate the computational model.

As the main objective of this study is to understand the effect of electrical stimulus over cartilaginous tissue, CHAPTER 4 presents a detailed review of the effect of biophysical stimuli on hyaline cartilage. The review is divided in two sections. On the one hand, the first section describes the application of electric and magnetic fields in in vivo and in vitro environments, focusing on cartilage explants and 3D constructs. On the other hand, the second section introduces the role of mechanical stimulation on hyaline cartilage such as compressive, tension, hydrostatic pressure and shear stress. 
The experimental approach containing the three different scenarios used in this work are presented from CHAPTERS 5 to 7. Thus, in CHAPTER 5, the cellular behavior of chondrocytes cultured in monolayer and stimulated with electric fields is presented. This chapter illustrates the construction of a coaxial capacitor, which allow determining the dielectric constants of the culture system. Moreover, a computational simulation to estimate the electric fields within the culture is performed. The change in cell proliferation and synthesis of glycosaminoglycans is highlighted after the cell culture was electrically stimulated.

Once the in vitro chondrocytes behavior after a stimulation with electric fields was studied, the effect of this biophysical stimulus on chondroepiphysis explants is evaluated in CHAPTER 6. In this chapter, femurs and humerus explants were electrically stimulated, and the response to this stimulation was measured histomorphometrically. In this context, the total length of the epiphyseal plate, and the length of the growth plate zones were analyzed. Moreover, other physiological and cellular parameters were measured such as cell density, columnar arrangement and columnar angles.

Finally, the potential effect of the application of electric fields in the chondrogenic process was assessed. Therefore, CHAPTER 7 shows the chondrogenic differentiation of mesenchymal stem cells cultured in hydrogels and stimulated with electric fields. This chapter describes the mechanical and dielectric characterization of hydrogels. Lastly, this section evaluates the in vitro cultures by analyzing the expression of chondrogenic markers after electrical stimulation.

The main conclusions derived from this work are presented in CHAPTER 8, altogether with some perspectives and recommendations for future works. 



\section{CHAPTER 2}

\section{Hyaline cartilage:}

structure, pathologies and therapeutic alternatives 



\subsection{Summary ${ }^{1}$}

This chapter highlights the morphophysiology, molecular structures, biomechanical properties, pathologies and treatments of the hyaline cartilage. Moreover, this section highlights the use of tridimensional structures to restore the articular cartilage, focusing on the development and application of hyaluronic acid - gelatin hydrogels.

\subsection{Hyaline cartilage}

Cartilage is an connective tissue that, depending on the features of the extracellular matrix (ECM) and the location in the human body, can be classified in three types: elastic, fibrillar and hyaline [1], [2]. For instance, elastic cartilage can be found in organs such as the ear and the epiglottis. This elastic tissue has a low concentration of collagen type II and it is mainly constituted by scattered elastic beams that form a threadlike network that provides elasticity to the cartilage. Similarly, the intervertebral disc and the meniscus are composed by fibrocartilage, which has a fibrous ECM rich in collagen type I. Finally, hyaline cartilage is a tissue rich in collagen type II and proteoglycans that can be found in two different parts of the bone. On the one hand, the articular cartilage is located at the end of the bones (epiphyses) [3]-[5], and its functional properties are reducing the friction coefficient and supporting of mechanical loads between opposing joint surfaces [6]. On the other hand, a cartilaginous layer, known as the growth plate, is located between the epiphysis and the diaphysis, and it is responsible of longitudinal growth of long bones [7], [8].

Since the hyaline cartilage in most of cases fulfills a damping function, their cellular and molecular structures are crucial to support mechanical loading. Accordingly, this type of cartilage is composed by one cellular

\footnotetext{
${ }^{1}$ Part of the review presented in this chapter has been published in: J. J. VacaGonzález, M. L. Gutiérrez, and D. A. Garzón-Alvarado, "Cartílago articular: estructura, patologías y campos eléctricos como alternativa terapéutica. Revisión de conceptos actuales". Rev. Colomb. Ortop. y Traumatol., vol. 31, no. 4, pp. 202-210, 2017.
} 
type: the chondrocyte. This cell is responsible for synthesizing and degrading the main molecules of the ECM such as collagen fibers and proteoglycans (PGs) [3], [9]. These molecules provide dimensionality, elasticity and strength to the cartilage in order to support mechanical loads [9], [10]. Considering that the articular cartilage and the growth plate are soft tissues presented in the skeletal system, the anatomy and the molecular structure of those tissues are summarized in the following sections.

\subsubsection{Embryological development of the articular cartilage and the growth plate}

Bone structure arises from a mesenchymal cell condensation during embryonic development through a process known as endochondral ossification (Figure 2-1) [4], [8]. This process begins when the mesenchymal stem cells migrate into the location of prospective long bone formation. After cell recruitment, the epithelium/mesenchymal interaction induces cellular condensation (Figure 2-1A). Once the condensation occurs, the mesenchymal stem cells proliferate and differentiate into chondrocytes forming a cartilaginous mold rich in collagens and PGs (Figure 2-1B). The cartilaginous mold, also known as anlage, subsequently undergoes in an ossification process where chondrocytes in the center of the anlage stop their proliferation process and differentiate into hypertrophic chondrocytes (Hc) (Figure 2-1C). These hypertrophic cells enable the invasion of blood vessels $(\mathrm{Bv})$ and the formation of the bone collar (Bc) (Figure 2-1D). Angiogenesis in the primary spongiosa promotes the migration of mesenchymal cells to the center of the anlage. These cells differentiate into osteoblasts and form the primary ossification center (POC) (Figure 2-1E). Remaining chondrocytes located between the POC and the proximal and the distal epiphyses arrange into columns forming the growth plates (GP) (Figure 2-1F). Due to cycles of chondrocyte hypertrophy, vascular invasion and osteoblast activity, the secondary ossification center (SOC) is formed in the epiphysis of bones. Chondrocytes that remain at the end of the epiphysis form the articular cartilage (Figure 2-1G) [3], [5]. 
During endochondral ossification, a series of molecular events are carried out, where several extracellular and adhesive molecules are synthesized by chondrocytes, such as aggrecan (Agc1) and collagen type II (Col2 $\alpha 1$-IIa) [11]. In addition to these molecular events, the spatio-temporal regulation of both the ECM and the differentiation process also depend on the action of many growth factors, transmembrane receptors and transcription factors. For instance, it has been shown that the Sonic Hedgehog (Shh) and its respective transmembrane receptor Patched-1 and -2 (Ptch1,2) promote the formation of the cartilaginous mold. The interaction between this morphogen and its receptor allows the recruitment of both the transmembrane protein Smoothened (Smo) and the Gly-type transcription factors. This signaling pathway is relevant since those factors activate SOX-9, one of the master regulators of chondrogenesis, and Nkx3-2 that maintains the expression of SOX-9 in condensing cells [12].

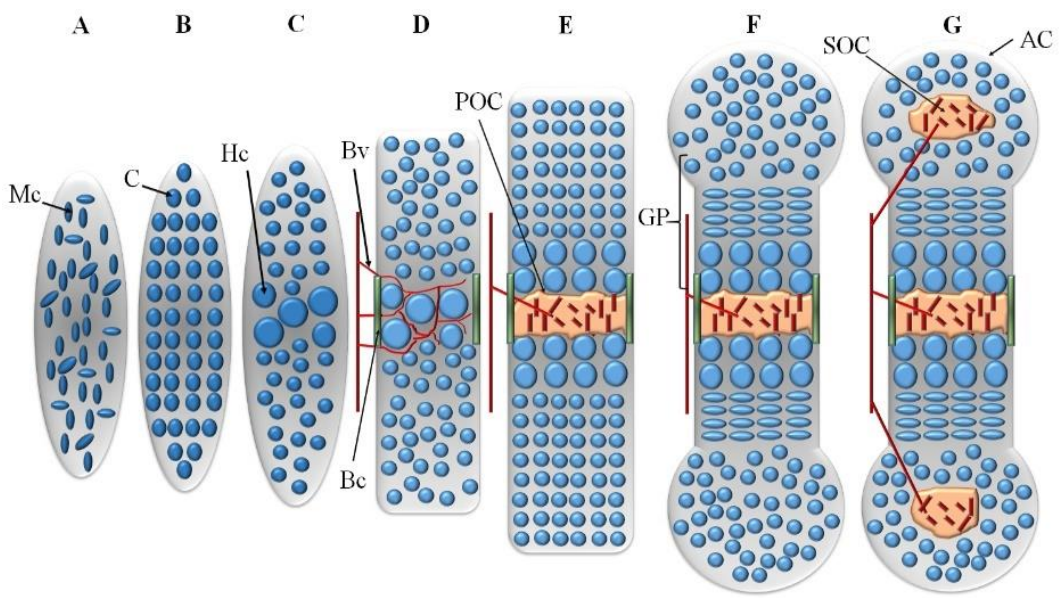

Figure 2-1: Endochondral ossification process.

\subsubsection{Anatomy of cartilaginous structures in long bones}

\subsubsection{Articular cartilage}

Articular cartilage is located in the synovial joints, at the end of the bones that form the joint. The thickness of the articular cartilage varies 
depending on its location. For instance, cartilage in interphalangeal joints have a thickness between 1 and $2 \mathrm{~mm}$, while in the knee or the hip joints, that support a greater weight, the cartilage have a thickness between 3 and $5 \mathrm{~mm}$ [5]. Articular cartilage acts as a shock absorber; therefore, this tissue is exposed to different mechanical loads such as tension, compression, and shear stress (Figure 2-2). On the one hand, tension loading exerts an outward force increasing body elongation. On the other hand, compression loading applies a thrust force that acts over the entire length of the tissue, making it flatter. Finally, shear loading is tangential to the body and deforms the tissue in several directions. Accordingly, hip and ankle joints support loads from 1 to $4 \mathrm{MPa}$, while knee joints support loads that vary from 5 to $10 \mathrm{MPa}$ during walking and $18 \mathrm{MPa}$ during physical activity [3], [4], [9], [13].

Cellular and molecular composition of the ECM in articular cartilage are fundamental to support mechanical loading. Chondrocytes represent approximately $1-5 \%$ of the total volume of the tissue [4], and their morphology varies according to their location inside the tissue (Figure 2-3A). Accordingly, chondrocytes can be round, oval or flattened [3], [4].

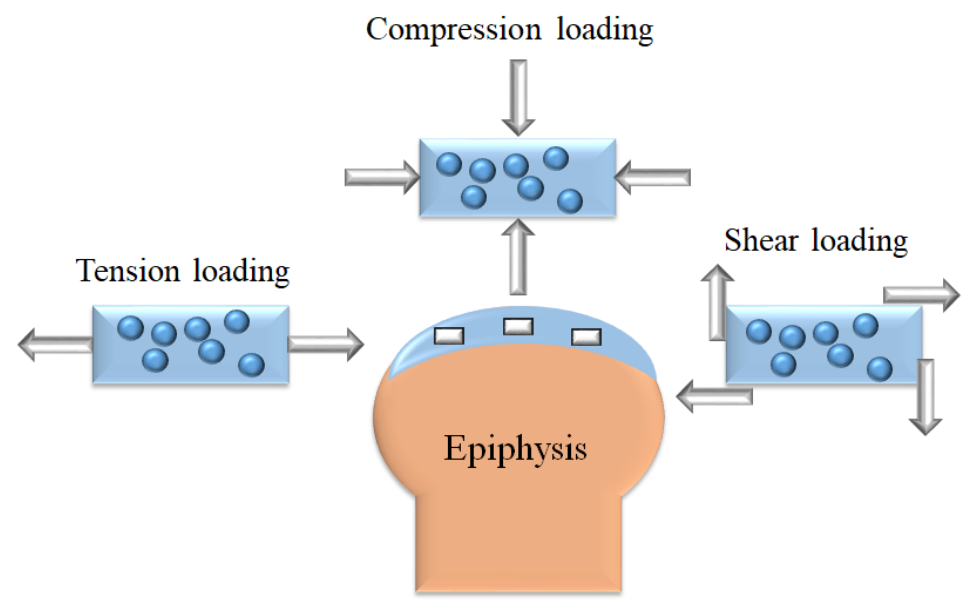

Figure 2-2: Mechanical loads supported by articular cartilage.

The ECM represents more than $95 \%$ of the volume of the articular cartilage, where $60-80 \%$ is water, $15 \%$ is composed of collagens, 
including collagen type II, VI, IX, X, XI, and 9\% is composed by PGs [1], [4]. Nevertheless, collagen and PGs percentage vary depending on their location within the articular cartilage (Figure 2-3B). Thus, articular cartilage is organized in zones according to cell morphology and collagen type II orientation (Figure 2-3A) [1], [10].
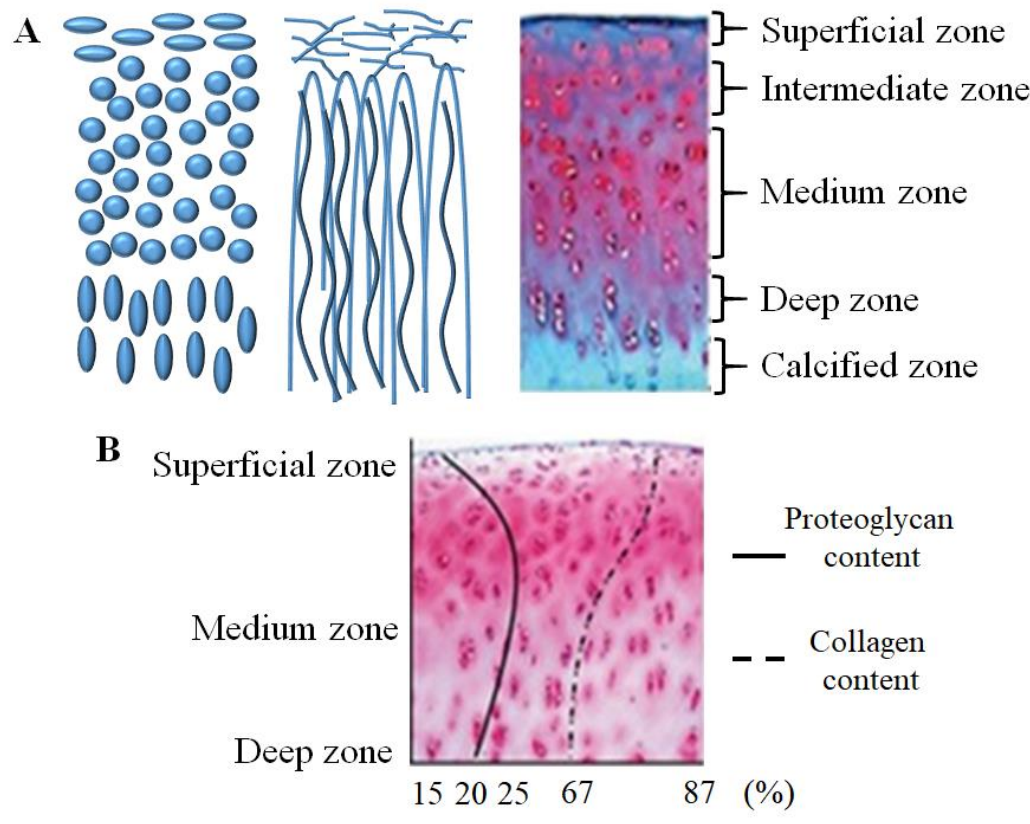

Figure 2-3: Scheme of the articular cartilage zones.

A) Chondrocyte morphology in different articular cartilage zones (left). Collagen fiber organization within the ECM (center). Articular cartilage zones (right). B) Collagen and PGs percentages inside articular cartilage. Modified of [14].

The most distant areas from the bone form the superficial area, constituting the thinner layer of the tissue (approximately 50 to $100 \mu \mathrm{m}$ ). This area has flattened chondrocytes, collagen fibers parallel to the joint surface (Figure 2-3A), and a low concentration of PGs (Figure 2-3B) [10]. Thereafter, a transition region sets out the boundaries of the intermediate zone, which has a lower cellular density of chondrocytes with an oval morphology. Followed by this region, the chondrocytes become rounder and form small clusters in the medium zone. In this 
zone, the chondrocytes actively synthetize aggrecan, the main PG found in hyaline cartilage, and collagen type II, which exhibit an arcade-like structure interspersed. In the deepest area, collagen is perpendicular to the joint and rather flattened chondrocytes are arranged into columns. Finally, it is in the calcified zone where chondrocytes get hypertrophic and synthesize collagen type $\mathrm{X}$ [14].

\subsubsection{Growth plate}

The growth plate, also known as the epiphyseal plate or the physis, is the tissue located in the metaphysis of long bones. It is histologically arranged in four zones: reserve, proliferative, pre-hypertrophic and hypertrophic (Figure 2-4) [15].

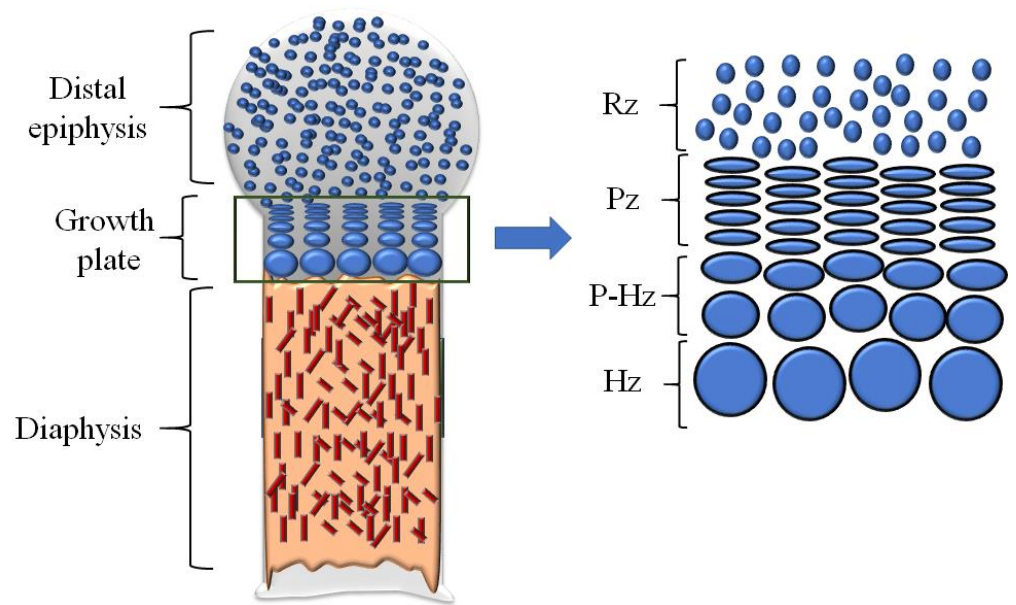

Figure 2-4: Scheme of the growth plate zones.

The reserve zone contains chondrocytes with rounded morphology which are randomly distributed. Moreover, these cells are characterized for having a low proliferative capacity [7], [15], [16]. The proliferative zone has flattened chondrocytes arranged into columns, which are parallel to the axis of bone growth [7], [15]. The pre-hypertrophic zone has aligned columnar chondrocytes that have started the maturation process to get hypertrophy. Finally, the hypertrophic zone contains chondrocytes in terminal differentiation to mineralize the tissue. In this zone, cells stop proliferating and increase their size ten times compared 
with chondrocytes in the reserve zone [7], [8], [16]. A continuous cell dynamic within the growth plate maintain the longitudinal growth of the bone constant. Moreover, the growth plate experiences morphological changes over time. For instance, the physis in humans is concave at the age of four, while it becomes straight at the age of seven. Lastly, the growth plate assumes an arch form at the beginning of the puberty [17]. Besides the width of the growth plate changes through life. At early stages of development the epiphyseal plate is wider, while at the end of adolescence the thickness of the physis decreases progressively until it is completely resorbed connecting the epiphysis with the diaphysis [16].

\subsubsection{Molecular components of the articular cartilage and the growth plate}

\subsubsection{Articular cartilage}

\section{Collagen fibers}

The main component of the ECM in the articular cartilage is collagen type II. This is a fibrillar protein synthetized as a monomer, assembled as a trimmer and extracellularly processed to remove the amino and carboxy terminals [10]. Collagen fibers align to provide stiffness to the cartilage depending on the direction of the supported load by the joint [14]. Accordingly, the articular cartilage has five types of collagen [9], [10]. The most important is collagen type II that constitutes the bulk of total collagen in the articular cartilage (about 80\%). This type of collagen is responsible for providing a high resistance to cyclic compression. Collagen type VI is concentrated around the chondrocytes, helping them to adhere to the ECM. This collagen composes $5 \%$ of total collagen in the articular cartilage. Collagen type IX facilitates the interaction between collagen fibrils and PGs, and it composes $15 \%$ of total collagen in the articular cartilage. Collagen type $\mathrm{X}$ organizes the collagen fibers in a tridimensional hexagonal lattice in the calcified zone of the articular cartilage, and it represents $5 \%$ of total collagen (Figure 2-3A). Last, collagen type XI regulates collagen fiber size, forming a pericellular network around the chondrocytes. 
Moreover, it also allows collagen type II production, representing $15 \%$ of total collagen.

\section{Proteoglycans (PGs)}

The PGs are very high molecular weight molecules, which provide support and dimensionality to the cartilaginous tissue. PGs are constituted by a core protein known as hyaluronic acid with glycosaminoglycans (GAGs) that are perpendicularly attached through covalent bounds with a link protein (Figure 2-5) [10]. These long chains of linear carbohydrate polymers are negatively charged under physiological conditions due to their sulfate and uronic acid groups. Negatively charged sulfates attract positive ions, such as sodium, that in turn attract water. Thus, the articular cartilage is highly hydrated allowing the cells to maintain a distance between them. Moreover, the PGs provide an osmotic resistance capacity and compressibility to the tissue [4]. Additionally, PGs are joined to the chondrocyte cell membrane not only acting as signal modulators in communication processes between cells and their environment [3], but also promoting cell adhesion [4].

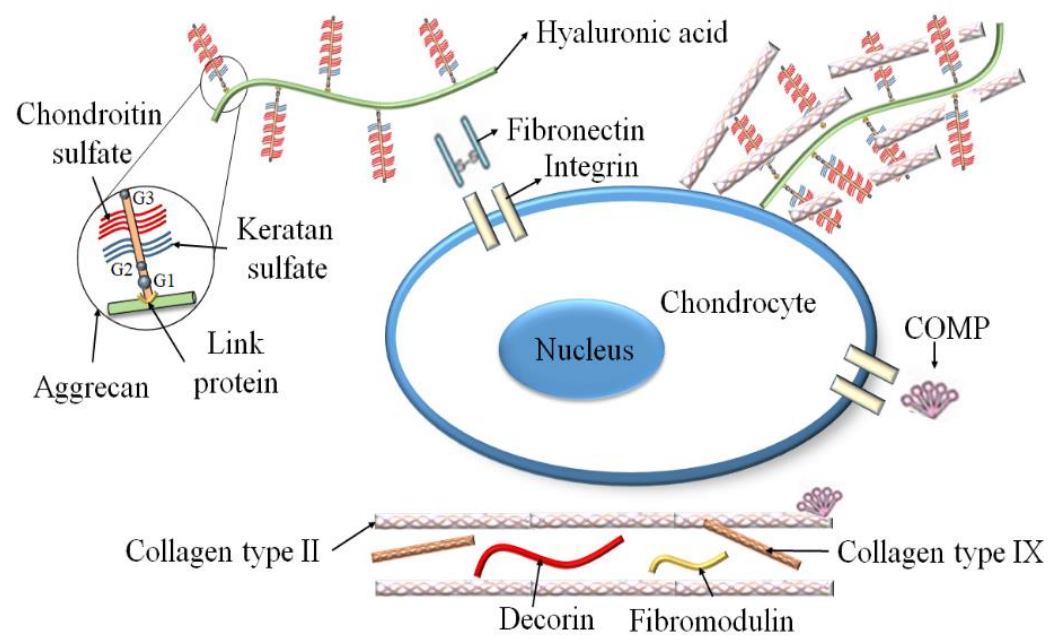

Figure 2-5: Representative scheme of PGs, collagen fibers and GAGs.

Modified of [10]. 
The main PG in the articular cartilage is the aggrecan; it is composed by a central protein of 210 to $250 \mathrm{kDa}$ with three globular domains: two in the amino terminal (G1 and G2) and a third one in the carboxy terminal (G3). This third domain is separated by a region of two GAGs: the keratan sulfate (KS) and the chondroitin sulfate (CS) (Figure 2-5) [4], [10]. The main functions of the globular domains are to provide aggregation, cell adhesion and chondrocyte apoptosis support, and supply hyaluronic acid binding sites [18]. Aggrecan can interact with other proteins such as collagen type II, connecting the ECM with all constituents of the cellular surface [10]. Additionally, decorin and fibromodulin are PGs with lower molecular weight, linked with collagen type II. Other molecules located in cartilage matrix are fibronectin and cartilage oligomeric protein (COMP). The former is a glycoprotein that binds with cartilage membrane receptors, while COMP mediate the interaction of chondrocytes with the cartilage matrix through interaction with cell surface integrin receptors. Moreover, COMP maintain the structural integrity of cartilage interacting with other ECM proteins such as collagen type II (Figure 2-5) [19], [20]. These smaller PGs play important roles determining collagen fiber physical properties [1].

\section{$\underline{\text { Transcription factors }}$}

The transcription factor SOX-9 is a molecule member of the SOX family. This transcription factor is essential for the differentiation of mesenchymal condensations into chondrocytes. Moreover, the SOX-9 is crucial for the synthesis of characteristic molecules of cartilage matrix such as collagen type II, IX, XI and aggrecan [21]. During long bone development SOX-9 is synthetized by proliferative chondrocytes and it is inhibited by hypertrophic chondrocytes [22]. Studies have demonstrated that SOX-9 in presence of SOX-5 and SOX-6 regulates the endochondral ossification process [23]. Another important transcription factor is RUNX2, this molecule also known as Cbfa1 is present in growth plate chondrocytes and it is responsible for the differentiation of proliferative chondrocytes into hypertrophic [24]. 


\subsubsection{Growth plate}

Although the growth plate shares some molecular factors present in the articular cartilage, the function of the epiphyseal plate, in controlling the growth rate of the long bones, is also regulated by several molecular factors. Among the most relevant factors are the systemic hormone levels, local growth factors and signaling molecules (Figure 2-6).

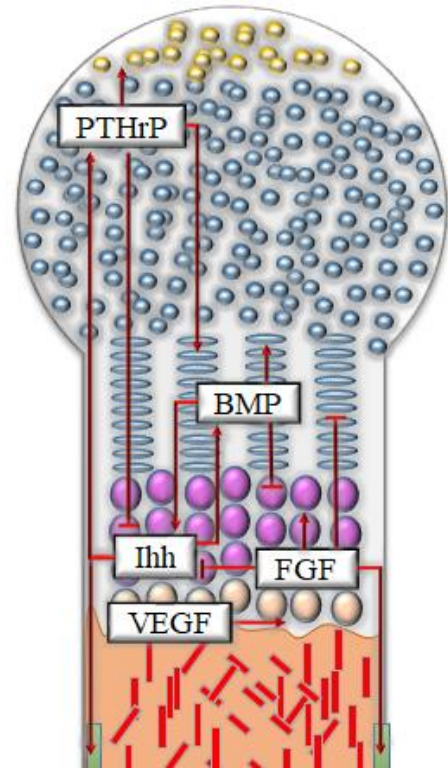

Figure 2-6: Schematic representation of molecular regulations in the growth plate.

\section{Indian hedgehog/Parathyroid Hormone-related Protein}

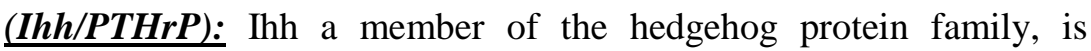
expressed by pre-hypertrophic chondrocytes during long bone development [25]. Besides, PTHrP is a protein synthetized by proliferative chondrocytes during the fetal stage in the periarticular perichondrium at the end of the anlage [26]. Accordingly, PTHrP acts over proliferative chondrocytes to keep them proliferating and simultaneously inhibits the synthesis of Ihh. Thus, if PTHrP activity is sufficiently distant from the proliferative zone, the production of Ihh will start in the pre-hypertrophic zone. Ihh acts on proliferative 
chondrocytes to promote their proliferation through PTHrP synthesis stimulation [21], [27].

Vascular endothelial growth factor (VEGF): VEGF is a signaling protein involved in angiogenesis [28]. VEGF is synthetized in the growth plate by hypertrophic chondrocytes located in the ossification front. Once cells synthetize VEGF, a blood vessel formation appears within the ossification front of the growth plate [27], [29], [30]. These blood vessels have an important role within the growth plate as they not only allow the entry of growth factors towards the physis, but also trigger an oxide and dioxide flow to control chondrocyte apoptosis. Moreover, the angiogenesis promotes the entry of progenitor cells (undifferentiated mesenchymal stem cells) from the primary ossification center to the epiphysis [31].

Bone morphogenic proteins (BMPS): BMPs are glycoproteins of low molecular weight characterized by interacting with membrane receptors (BMPR). BMPs regulate proliferation process, downregulate the maturation process of chondrocytes and promote Ihh synthesis [27]. BMPs trigger different signaling pathways that control several events such as chondrogenesis, chondrocyte proliferation, blood vessel formation and mineralization [32]. Regarding the chondrogenesis process, it has been demonstrated that BMP 2 plays a pivotal role in cartilage regeneration due to the fact that it promotes SOX-9 and aggrecan synthesis [33].

Fibroblast growth factors (FGFs): FGFs repress chondrocyte proliferation, differentiation and Ihh synthesis during the endochondral ossification process [27]. It has been observed that FGF 1 is expressed by pre-hypertrophic chondrocytes and perichondrium, FGF 2 is synthetized in the perichondrium and primary ossification center after mesenchymal condensation, and FGF 3 is expressed by proliferating chondrocytes. Moreover, some studies have shown that FGF 3 knockout increases cell proliferation and allow the expansion of columns within the growth plate [21]. 


\subsubsection{Biomechanical properties of hyaline cartilage}

Hyaline cartilage is a biphasic material which consists of an interstitial fluid phase and a porous-permeable solid phase [10]. On the one hand, the properties of the liquid phase are given by the PGs, since they are negatively charged and allow the retention of water molecules within the tissue. On the other hand, the solid phase is provided by the distribution of collagen fibers, since they are strategically interlaced to give three-dimensionality to the cartilage [34]. The solid and fluid phases are crucial in distributing homogeneously external loads over the entire ECM when the cartilage is subjected to compression, shear and/or tension loads. This distribution is achieved by the permeable porous phase, which allows the flow of fluids that in turn creates a load transfer between both phases together with a pressurization of the interstitial fluid [35]. Accordingly, the cartilage intrinsic characteristics determine the main properties of the tissue such as compression resistance, tensile strength, shear stress resistance, hydrostatic pressure, swelling degree, permeability and viscoelasticity.

Compression: The development and maintenance of hyaline cartilage are closely correlated to the effect of the mechanical load. The compression loading is crucial for preservation of cartilage structure, because this tissue is loaded primarily under compression. This load is supported by the solid phase of the cartilage, the fluid pressure and the nearly impermeable subchondral bone [36]. A volumetric change on cartilage structure occurs when the tissue is compressed. This internal morphologic change results in the flow of interstitial fluid, which generates a significant frictional resistance within the tissue [34].

Tensile strength: The tension load describes the maximum stress supported by a material when is stretched. The cross-linked collagen in cartilage is a network that is responsible for supporting the tensile strength. When a tension loading is applied to the tissue, the collagen fibers tighten and align in order to absorb the tensile force [37].

Shear stress: The load perpendicularly applied to the cartilage surface is known as shear stress. This tangential load internally deforms the 
cartilage in an angular way affecting the inner zones of the cartilaginous tissue. The interaction between collagen fibers and PGs supports and distributes the shear stresses within the tissue. Collagen fibers, having a more elastic behavior than PGs, enable the absorption of shear loads. Consequently, this absorption reduces the fractional dissipation and the phase angle [35].

Hydrostatic pressure: The fluid phase of the articular cartilage is in charge of supporting the hydrostatic pressures generated by compressive loads. These hydrostatic pressures are not uniform within the cartilage because the applied loads are heterogeneous across the tissue; therefore, a gradient in total stresses and pressures are formed, particularly near the joint surface. The hydrostatic pressures vary depending on the cartilage location; for example, the cartilage in the knee joints support hydrostatic pressures between 3 and $10 \mathrm{MPa}$, while the cartilage in the hip joints support hydrostatic stresses of $18 \mathrm{MPa}$ [38].

Swelling: Swelling is the mechanisms by which the residual stresses occur within the cartilage. Residual stresses and strains are physical magnitudes generated inside the cartilage after mechanical loading. These residual loads play a key role within the tissue enhancing cartilage physiological behavior and reducing the stresses when the tissue is loaded under physiological conditions [35]. Swelling is generated by the ionic constituents of the synovial fluid and the negatively charged GAGs (keratan sulfate and chondroitin sulfate) associated with the PGs molecules in the solid matrix of the cartilage [39].

Permeability: Permeability is a property of porous materials, which is measured as the amount of fluid that can flow through a material. Particularly, permeability in hyaline cartilage is given by the PGs because they are the molecules that resist to fluid flow. It has been evidenced that a decrease in PGs concentration in the bone-cartilage interface lead to an increase of the permeability in the cartilage surface [40]. Moreover, it has been demonstrated that the orientation of the collagen fibers also influences the permeability of the tissue, due to the 
fact that permeability decreases in zones where the fibers are parallel to the surface [41]. Permeability in the knee cartilage of humans is between $1.14 \times 10^{-15}$ to $2.17 \times 10^{-15} \mathrm{~m}^{4} / \mathrm{Ns}$ [34].

Viscoelasticity: A material is viscoelastic when a constant load or strain is applied, and its response varies with time. In cartilage, this property is determined by the storage $G^{\prime}$ and the loss $G^{\prime \prime}$ moduli. The $\mathrm{G}^{\prime}$ represents the capacity of the tissue to store energy for elastic recoil, while $\mathrm{G}^{\prime \prime}$ characterizes the ability of a cartilage to dissipate energy [42]. Morphophysiologically, the collagen fibers and the fluid flow are responsible for determining the viscoelasticity of the cartilage. The cross-linking of the collagen fibers increases the viscoelastic shear modulus, while the fluid flow plays an important role as loading support in confined compression [43].

\subsection{Pathologies affecting hyaline cartilage}

When hyaline cartilage advances in age, it is conditioned to internal wear caused by the mechanical loads that are supported by the tissue. These mechanical loads generate morphological and functional alterations [44]. However, there are some injuries and diseases that may prematurely occur by reason of genetic, growth, metabolic, and/or traumatic factors [45].

There are three types of injuries that affect cartilage. First, there are lesions that disrupt and degrade the ECM of the tissue. However, the remaining viable chondrocytes increase their synthetic activity and repair the affected tissue. Second, there are changes in cartilage thickness that generate fissures in the structure of the cartilage. Last, there are injuries affecting the entire thickness of the cartilage that reach the subchondral bone. A possible recovery for this type of injury is carried out by progenitor cells migrating from the bone marrow to the injured tissue, generally replacing the tissue with fibrocartilage. This fibrillar tissue is mostly inefficient for load bearing and has a loss in friction properties [46]. According to the mentioned above and depending on the location of the hyaline cartilage, several pathologies may affect the cartilaginous tissue. 


\subsubsection{Articular cartilage}

Osteoarthritis (OA) is the most frequent degenerative pathology of the articular cartilage. In this pathology both chondrocytes and ECM are affected [11], [47], [48]. Studies have revealed that OA affects both men and women usually over 60 years old. The OMS speculates that this disease will be the fourth cause of disability in the world by 2020 [49]. OA involves several factors that include mechanical damage of the joints, inflammation, and genetic predisposition [50]. It is manifested with high morphological, biochemical, molecular and biomechanical changes that result in an imbalance between synthesis and degradation of ECM components in articular cartilage. Thus, alterations of collagens and PGs decrease the tensile strength and the stiffness. The pathology leads to a decrease in thickness of the articular cartilage, subchondral bone remodeling and chronic inflammation of the synovial membrane [45], [51], [52].

Other pathologies such as neurogenic arthropathy, spondyloarthritis and osteochondritis affect the articular cartilage generating pain, inflammation, movement difficulties, fragmentation and joint fractures [53]. Overall, pathologies modifying morphology, composition and biomechanical properties of the articular cartilage may have an important impact on the quality of life of elder population. Therefore, there is a necessity to either develop appropriate treatments or find physiotherapeutic alternatives to restore cartilaginous tissue from lesions.

\subsubsection{Growth plate}

Pathologies that affect the growth plate tissue in turn affect the shape and the length of several skeletal elements. The main diseases that alter the epiphyseal plate are generated either by genetic disorders (skeletal dysplasias) or induced malformations during gestation [54]. Skeletal dysplasias such as osteodysplasias, osteochondrodysplasias and chondrodysplasias are the most common pathologies that affect cartilage and bone development [55]. Regarding the cartilaginous tissue, chondrodysplasias are pathologies that disturb the ECM of the 
epiphyseal plate. Such disturbances are caused in many cases by genetic alterations that modify the structure and synthesis of proteins, transcription factors, morphogens and enzymes among others [54]. These modifications are reflected in bone alterations that cause dwarfism and joint dysfunctions. One example of a genetic chondrodysplasia is the mucopolysaccharidosis type IV (MPS IV). This disease is generated by the alterations in the catabolism of the GAGs present in the cartilage ECM (keratan sulfate and chondroitin sulfate). MPS IV is caused by mutations in the genes coding for lysosomal enzymes that degrade GAGs. This leads to the accumulation of undegraded polysaccharides, in both cytoplasm and ECM, that results in bone deformities [56].

\subsection{Treatments to restore hyaline cartilage}

Since different pathologies affect and degrade cartilage tissue, some therapeutic treatments have been implemented either to relieve the pain locally in the joint or to replace the affected articular surface with new cartilaginous tissue. For this reason, alternatives have focused on restoring articular cartilage through invasive and noninvasive treatments.

\subsubsection{Noninvasive treatments}

Physiotherapy makes use of noninvasive alternatives to treat injuries in the joints. This type of treatment is based on the application of different physical stimuli over the injured tissue. The most commonly used treatments are: ultrasound (stimulation with frequency waves), magnetotherapy (application of static magnetic fields), electrotherapy (application of external electric fields), neuromuscular electrical stimulation and transcutaneous electrical nerve stimulation (TENS) [47]. According to the Osteoarthritis Research Society International (OARSI), therapies such as ultrasound, electrotherapy, neuromuscular electrical stimulation and TENS are treatments that need to be complemented with biomechanical interventions using knee braces, foot orthoses, strength training, weight management and physical exercise to relief pain in the knees with OA [57]. 
Although the non-invasive mechanisms used to treat cartilage have reduced the symptoms caused by the different diseases, a treatment that prolongs tissue healing has not yet been achieved. In fact, it has been demonstrated that several non-invasive therapies are of uncertain adequacy since they do not generate any effect in reducing local pain [57]. Accordingly, cartilage pathologies evolve to chronic states and in several cases the only solution is a surgical intervention.

\subsubsection{Invasive treatments}

The main purpose of invasive treatments is to replace osteochondral defects with a tissue that has the same characteristics of hyaline cartilage. One of the treatments is bone marrow stimulation, which consists in performing microfractures through the articular cartilage surface until the subchondral bone. These fractures allow the migration of mesenchymal cells to the affected site, and possibly their differentiation into chondrocytes to repair the lesion [1]. However, it is known that as the cells are not properly stimulated, the new formed tissue is generally fibrocartilage that will degenerate with time [46].

Another possible treatment uses autologous tissue transplantation, also known as mosaicoplasty. This treatment consists of taking small cylinders of healthy tissue from the edges of the articular cartilage, and inserting them into small holes previously drilled in the injured tissue [4]. Similar treatments are tissue grafting, which consists in taking small fragments of healthy articular cartilage from other joints of the body and inserting them into the affected cartilage. Osteotomy is another invasive technique, which consist in removing part of the bonejoint surface to reduce loads [3], [9].

Tissue engineering techniques have aimed to repair cartilage by autologous chondrocyte implantation (ACI), resulting either in regenerating or replacing the injured tissue [58]. ACI is a technique that consist in expanding the patient's chondrocytes through in vitro cultures. Once a sufficient number of chondrocytes have been obtained, these cells are re-implanted into the injured articular cartilage [59]. The use of scaffolds may enhance ACI, as these 3D structures play a pivotal 
role in the regulation of the mechanical loads that the cells receive, affecting their proliferation, differentiation, and maintenance of the chondrocytic phenotype. Moreover, scaffolds act as temporal ECM until the cells proliferate and start to synthetize the characteristic molecules of the tissue [60].

The development of scaffolds in cartilage tissue engineering is nowadays a challenge because finding a biomimetic material that has similar molecular properties to the hyaline cartilage has proved difficult. Accordingly, several studies have focused on the development of biocompatible matrices mainly composed by collagen and GAGs, as they represent $95 \%$ of cartilage ECM [1]. Moreover, collagens provide high resistance to cyclic compression, while GAGs such as hyaluronic acid retains water within the tissue ( $60 \%-80 \%$ of total cartilage volume) and provides compressive strength properties to the cartilage [4], [14].

Recently, hydrogels are used to mimic cartilage tissue because these scaffolds are produced with biocompatible natural polymers that resemble natural soft tissues. These matrices are composed by a combination of polymeric networks insoluble in water with a wellstablished cross-linked structure [61]. Hydrogels are constituted by specialized materials with high permeability properties, which facilitate the fluid of oxygen, nutrients and biomolecules through the scaffold. Moreover, these non-cytotoxic 3D structures are suitable for the encapsulation of cells before cross-linking in situ. These features make the hydrogels an attractive structure to be used in cartilage tissue engineering.

\subsection{Hydrogels used for hyaline cartilage regeneration}

Hydrogels are composed by cross-linked hydrophilic polymeric chains. The morphologic organization and the physicochemical composition of hydrogels favor the retention of water molecules within the structure, while the crossing points between the polymeric chains avoid the dissolution of the hydrogel in aqueous solutions [62]. Hydrogels also provide a tridimensional structure that facilitates the biophysical stimulation for the cells to acquire an ideal morphology. 
Depending on the polymeric materials used for the synthesis of hydrogels, different structures with specific properties can be obtained to be used in several applications [63]. For instance, cross-linking grade and biodegradability are factors that, depending on the monomer employed and the synthesis process used, have direct influence in both physicochemical properties such as water retention and mechanical properties [62]. Hydrogels can be fabricated using synthetic or natural polymers. On the one hand, synthetic polymers present advantages regarding mechanical properties; however, they represent disadvantages due to a lack of interaction between cell-scaffold and their grade of cytotoxicity after degradation. On the other hand, natural polymers mimic the ECM of soft tissues and increase adhesion, proliferation and differentiation of cells without toxicity issues. Most hydrogels are made either by proteins such as collagen, gelatin, elastin and fibrin or by polysaccharides such as hyaluronic acid, alginate, agarose, cellulose and chitosan [35].

The aforementioned proteins and polysaccharides are present in the ECM of natural tissues; therefore, cartilage tissue engineering is focusing on the development of 3D structures that contain these types of materials to further mimic and regenerate the cartilaginous tissue. Accordingly, some studies have shown that combined hydrogels of proteins-polysaccharides are suitable to maintain cell morphology and enhance protein synthesis. For instance, collagen scaffolds have been used for cartilage regeneration and the results have shown that collagen type I hydrogels lead the cells to undergo in distinct chondrogenic differentiation pathways [64], [65]. Moreover, in vivo experiments have evidenced that collagen scaffolds may be used for autologous transplantation to recover articular cartilage defects [66]. Another protein used to make hydrogels is gelatin. This protein is derived by breaking the natural triple-helix structure of collagen into single-strand molecules by hydrolysis [61]. Gelatin is considered as a biomimetic material because it presents several advantages such as biodegradability, low immunogenicity and low manufacturing cost. Gelatin is usually combined with polysaccharides to increase its biodegradability and biocompatibility properties. For example, gelatinalginate membranes demonstrated to have high hydrophilicity, 
permeance, permselectivity and a high swelling degree, indicating that higher concentrations of gelatin favor the transport of water molecules [67]. The combination of gelatin and hyaluronic acid has been also studied and the results have shown that this mixture constitutes a biocompatible and biodegradable substrate that increases gene synthesis of collagen type II, aggrecan, SOX-9, TGFbeta1, and TIMP1 [68].

\subsubsection{Hyaluronic acid-gelatin hydrogels}

\subsubsection{Hyaluronic acid}

Hyaluronic acid is a family member of GAGs and is found in several biological tissues. This polysaccharide is formed by long chains of disaccharides such as D-glucuronic acid and $\mathrm{N}$-acetyl glucosamine linked by $\beta$-1,3-glycosidic bonds (Figure 2-7) [69].

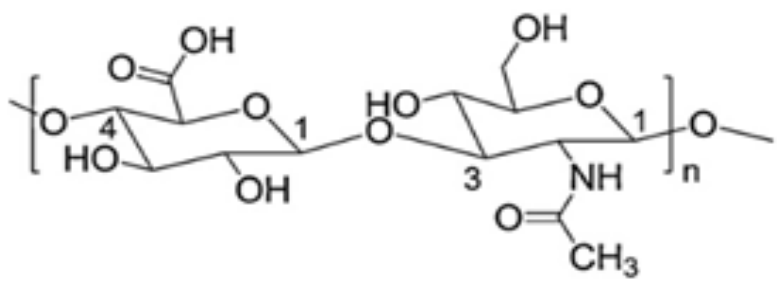

D-glucoronic acid $\mathrm{N}$-acetyl-glucosamine

Figure 2-7: Chemical structure of hyaluronic acid. It is constituted by a repetition of units of $D$-glucuronic acid and $N$-acetyl glucosamine. Modified of [69].

Hyaluronic acid stands out as a water-soluble non-toxic polymer. This polysaccharide is also enzymatically degradable by hyaluronidases, which cleaves in a single glycosidic bond on the hyaluronic acid backbone causing fragmentation [70]. Hyaluronic acid presents two chemical groups that are used for hydrogel formation: the hydroxyl and the carboxyl groups, respectively. These groups can be chemically cross-linked using different cross-linkers such as carbodiimides, e.g. 1ethyl-3-(3-dimethyl aminopropyl) carbodiimide/N hydroxysuccinimide 
(EDC/NHS), hydrazides, aldehydes, divinyl sulfone, photocrosslinking, autocrosslinking and enzymes [71], [72]. The chemical modification used by these stabilizers and activators regulate the fabrication of non-toxic hydrogels, which are suitable for cell culture and its gelation in situ [63].

\subsubsection{Gelatin}

Gelatin is a natural polymer obtained from the denaturation and the partial hydrolysis of collagen, presenting the same peptide sequences as natural collagen [61]. Gelatin is formed by the combination of amino acids such as glycine, proline and hydroxyproline (Figure 2-8) [73]. These amino acids are linked by peptide bonds forming polymer chains of molecular weights between $10000 \mathrm{~g} / \mathrm{mol}$ and $40000 \mathrm{~g} / \mathrm{mol}$ [74]. The physical properties of gelatin vary according to its melting point. For instance, below its melting temperature $\left(23^{\circ} \mathrm{C}\right)$, gelatin has helical chains, which act as cross-linking points. Nevertheless, these helical chains are unstable when gelatin is above melting temperature, which leads to a weakening of the intramolecular bonds and to the dissolution of the polymer in water [75].

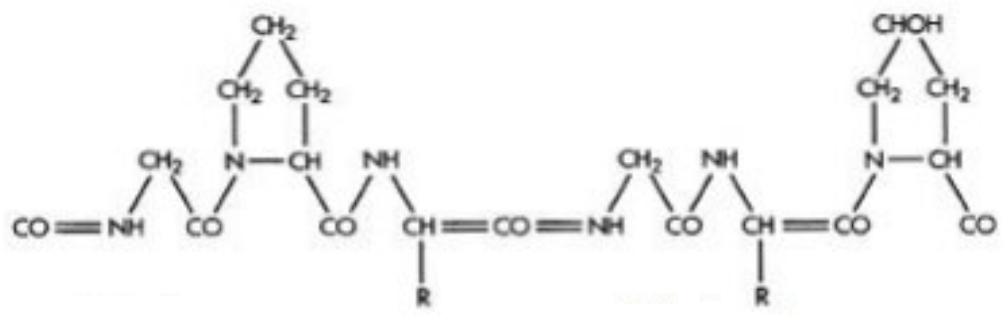

Glycine- Proline-X

Glycine-X Hydroxyproline

Figure 2-8: Chemical structure of gelatin. It is constituted by a repetition of units such as glycine, proline and hydroxyproline. Modified of [73].

Gelatin is a non-toxic biocompatible polymer, which may be enzymatically degraded by collagenase. However, the low mechanical resistance limits its direct application, so it is usual to use gelatin in 
combination with other polymers [76]. The physiological environment and the body temperature represent a problem in the solubility of gelatin; therefore, a chemical cross-linking is required to enhance the physicochemical properties of gelatin [77].

\subsubsection{Enzymatic cross-linking of hyaluronic acid-gelatin}

The cross-linking of hyaluronic acid and gelatin is achieved by chemical modification adding phenol groups to the carboxyl groups $(\mathrm{COOH})$ of the hyaluronic acid and gelatin. Thereafter, a cross-linking agent is added for hydrogel reticulation. The incorporation of phenol groups is carried out by adding tyramine, a monoamine that acts in the human body as vasoactive. Tyramine is bound to the carboxyl groups of hyaluronic acid and gelatin through the activator N-(3Dimetilaminopropil)-N'-etilcarbodiimide (EDC) and the stabilizer NHidroxisuccinimide (NHS). The reaction between EDC-COOH generates an O-acylisourea (Figure 2-9) [78].

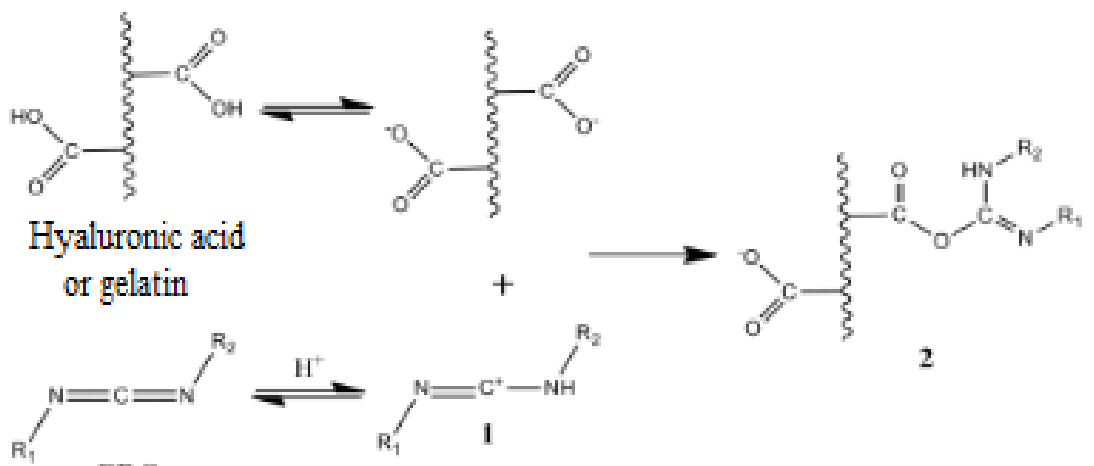

EDC

Figure 2-9: Reaction of hyaluronic acid or gelatin chains to form EDC carbocation (1) and O-acylisourea (2). Modified of [78].

Thereafter, the O-acylisourea reacts with both the NHS and the amine group of the tyramine in order to form an amide bond (Figure 2-10). This process induces the obtainment of hyaluronic acid and gelatin chains modified with phenol groups for subsequent enzymatic gelation [79]. 

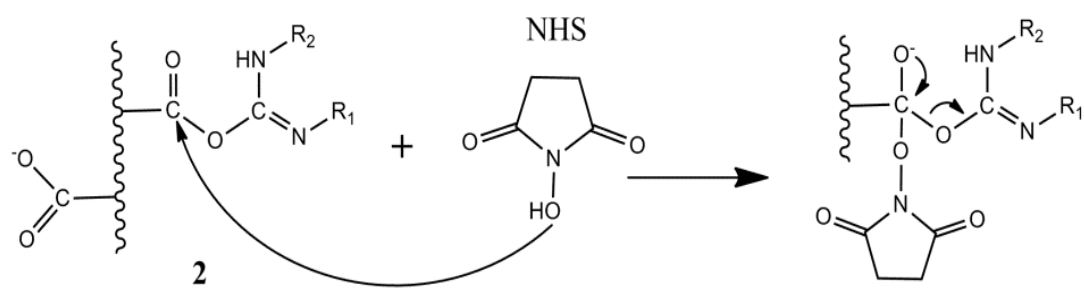

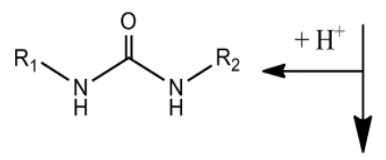
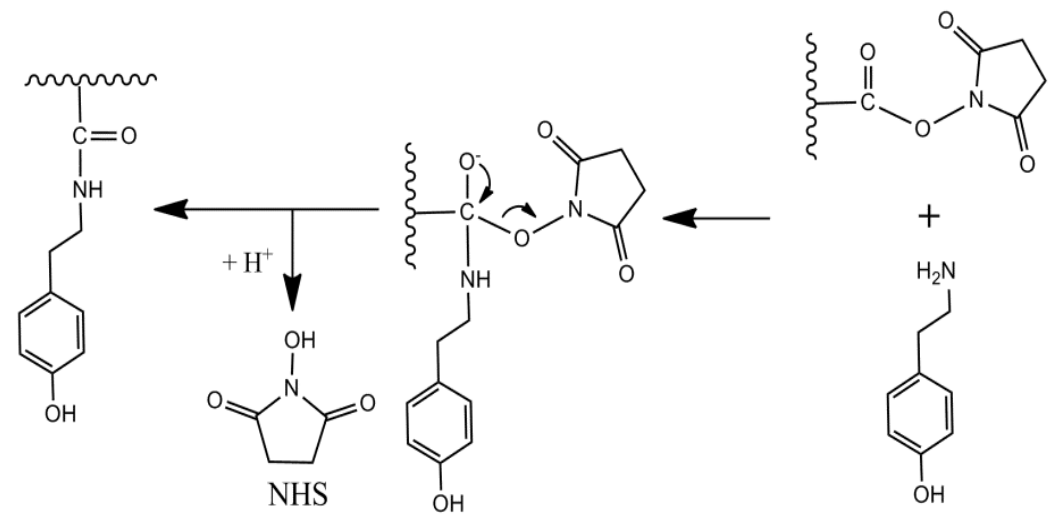

Tyramine

Figure 2-10: Representative scheme of the chemical reaction for obtaining hyaluronic acid or gelatin grafted with tyramine using $O$ acylisourea. Modified of [79].

Once the tyramine is grafted onto the polymeric chains, the crosslinking process is carried out by adding horseradish peroxidase (HRP), that catalyzes the cross-linking reaction and hydrogen peroxide $\left(\mathrm{H}_{2} \mathrm{O}_{2}\right)$, which acts as oxidizing agent of the tyramine molecules (Figure 2-11) [70]. 


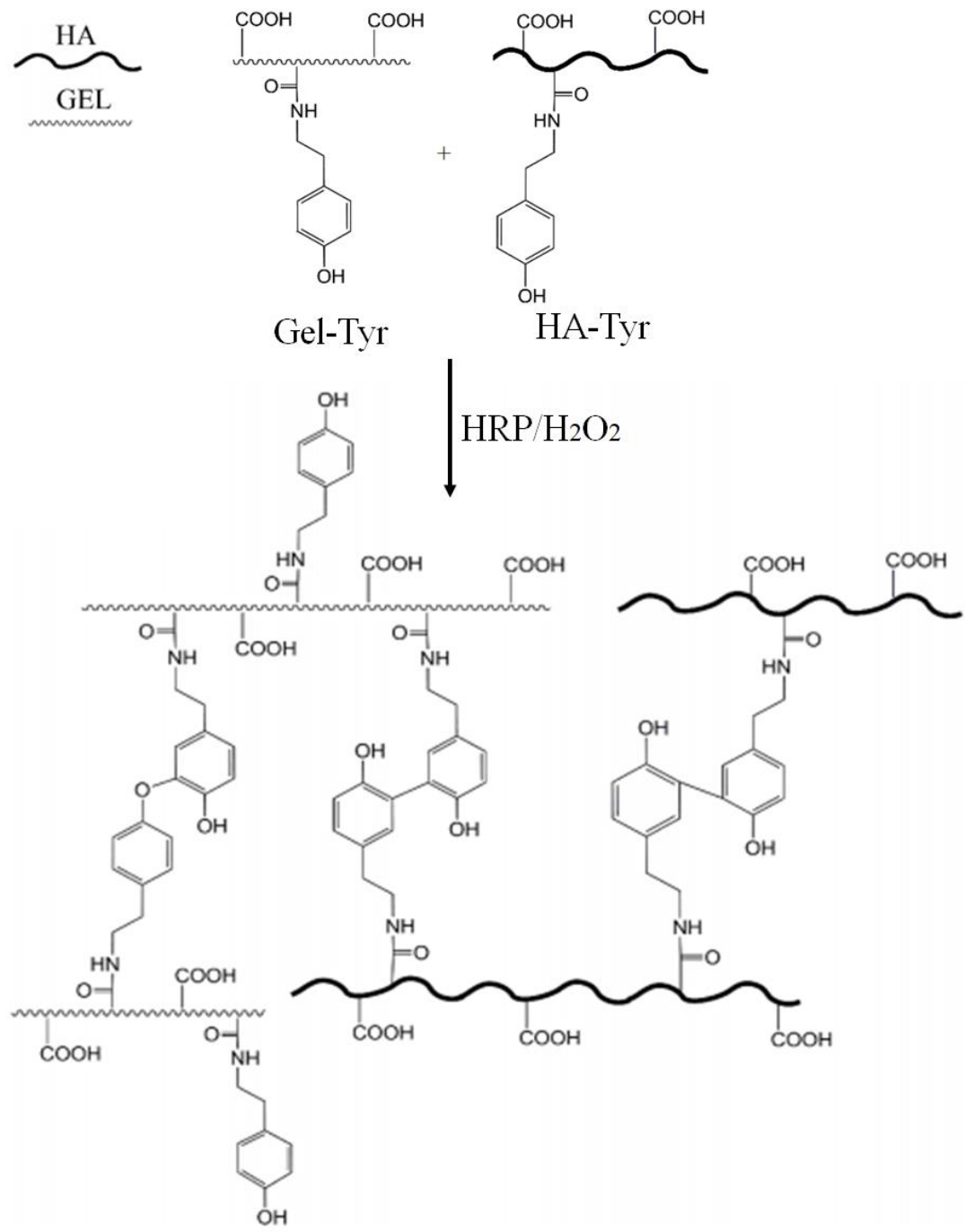

Figure 2-11: Diagram of the cross-link bonds formed in the gelation of hyaluronic acid and gelatin hydrogels using $\mathrm{HRP}$ and $\mathrm{H}_{2} \mathrm{O}_{2}$. Modified of [70].

\subsubsection{Advantages of hyaluronic acid-gelatin hydrogels}

Tyramine conjugates of hyaluronic acid and gelatin are injectable hydrogels formed by enzymatic cross-linking reactions. The main advantage of these hydrogels is that they are not cytotoxic and can be injected directly into the injured tissue. It means that the hydrogel can 
be inserted to fill the damaged zone before gelation and cover the affected area. This procedure can fill irregular shaped defects and the surgical interventions are less invasive compared with the surgeries to transplant preformed hydrogels [80]. Another advantage is that the cells can be seeded within the hydrogel before transplanting it. Once the hydrogel-cells mixture is done, the dissolution is located into the wounded tissue and cross-linked in situ. This procedure allows the inclusion of growth factors, proteins and other substances that will be homogenously distributed throughout the hydrogel once it is formed [61].

Hyaluronic acid has several biological functions; for instance, the receptors located in its chemical composition provide binding points between the cells and the ECM [81]. Additionally, this polysaccharide participates in both wound healing processes and the regulation of cell dynamics such as cell migration during angiogenesis, cell differentiation, proliferation and adhesion [82]. In the case of gelatin, this protein contains arginine-glycine-aspartic acid adhesion sequences, which are recognized by the integrins located in the cell membrane to promote cell adhesion [83]. The formation of this complex adhesions, also known as focal adhesions, trigger the formation of the actin cytoskeleton and the expression of genes involved in the processes of proliferation, differentiation and migration [84].

According to the aforementioned, a 3D structure composed by hyaluronic acid provides greater hydration and a better diffusion of nutrients. Moreover, the content of this polysaccharide not only increases the mechanical properties of the construct such as greater support to compression, tension and shear loads, but also enhances the physicochemical properties such as swelling degree, viscoelasticity and permeability [85]. Additionally, if the 3D structure contains gelatin, it will generate an ideal environment in which the cells can adhere to the ECM. In addition, gelatin promotes proliferation, differentiation, migration and synthesize of the characteristic molecules of a specific tissue. Overall, the combination of hyaluronic acid - gelatin is a 3D microenvironment rich in polysaccharides and proteins that provide water retention of molecules, resistance to compressive strengths and 
support to cyclic compression. This injectable hydrogel is a promising tool that may enhance cartilage tissue engineering approaches focused on cartilage restoration.

\subsubsection{Effect of hyaluronic acid - gelatin hydrogels in chondrogenesis}

Mesenchymal stem cells are promising cells that have been used to induce chondrogenesis, due to the fact that these cells have demonstrated a well-stablished differentiation towards a chondrocytic lineage [86]. The successful expression of different cartilage-specific markers makes mesenchymal stem cells an attractive cell type for treating articular cartilage defects. Nevertheless, the in vitro differentiation of these cells also promotes the expression of fibrocartilage-like molecules such as collagen type $X$ [87]. Taking this into consideration, there is a need to implement a three-dimensional construct that promotes chondrogenic differentiation by stimulating the production of molecules such as aggrecan, collagen type II and SOX-9 [85], [88]-[90]. In this context, hyaluronic acid - gelatin hydrogels have been implemented as a biomedical alternative to stimulate the chondrogenesis process. For instance, Angele et al., cultured human mesenchymal stem cells into three different compositions of hyaluronic acid/gelatin $(100 / 0 \%, 95 / 5 \%$ and $70 / 30 \%)$. The results indicated that hydrogels of $95 / 5 \%$ and $70 / 30 \%$ of polymer concentrations increased the contents of PGs and collagen type II [91]. Similarly, a study developed by Pfeifer et al., in which they used the same concentration of hyaluronic acid and gelatin previously described, showed that cell viability was higher at the beginning of the culture in hydrogels with a composition of 70/30\%, while the mixture of 5/95\% evidenced an increase in cell proliferation after 10 days of culture. Regarding the molecular production, it was evidenced that hydrogels of pure hyaluronic acid content (100/0 \%) yielded the best synthesis of collagen type II, while GAGs content increased over time within every scaffold composition [92]. A similar study was performed by Moulisová et al., in which bone marrow mesenchymal stem cells were cultured into different proportions of hyaluronic acid - gelatin (100/0 \%, 70/30 \%, $50 / 50 \%, 30 / 70 \%$, and $0 / 100 \%)$. The results varied in this study 
according to the culture media used for cell culture. For example, hybrids cultured in basal media experienced an increase in cell proliferation, in comparison with the scaffolds that were cultured in presence of growth factors such as TFG- $\beta 3$. On the other hand, the molecular expression of aggrecan and SOX-9 were higher in all mixtures using both basal media and chondrogenic media. It was also possible to observe that GAGs were synthetized in all hybrids, except in hydrogels that contained just gelatin. Finally, the expression of collagen type II was higher in hydrogels that have a composition of $70 / 30 \%$, and that were cultured using basal media [85]. Chen et al., implemented hyaluronic acid - gelatin hydrogels as a natural matrix for nucleus pulpous cells culturing. Results showed that hydrogels increased the genetic expression of chondrogenic markers such as SOX9, aggrecan and collagen type II [93]. 



\section{CHAPTER 3}

\section{Mechanobiological modeling of endochondral ossification}





\subsection{Summary ${ }^{2}$}

Long bone formation starts early during embryonic development through a process known as endochondral ossification. This is a highly regulated mechanism that involves several mechanical and biochemical factors. Because long bone development is an extremely complex process, it is unclear how biochemical regulation is affected when dynamic loads are applied, and also how the combination of mechanical and biochemical factors affect the shape acquired by the bone during early development. In this study we develop a mechanobiological model combining: 1) a reaction-diffusion system to describe the biochemical process and 2) a poroelastic model to determine the stresses and fluid flow due to loading. We simulate endochondral ossification and the change of long bone shapes during embryonic stages. The mathematical model is based on a multiscale framework, which consisted in computing the evolution of the negative-feedback loop between Ihh/PTHrP and the diffusion of VEGF molecule (on the order of days) and dynamic loading (on the order of seconds). We compare our morphological predictions with the femurs of embryonic mice. The results obtained from the model demonstrate that pattern formation of Ihh, PTHrP and VEGF predict the development of the main structures within long bones such as the primary ossification center, the bone collar, the growth fronts and the cartilaginous epiphysis. Additionally, our results suggest high load pressures and frequencies alter biochemical diffusion and cartilage formation. Our model incorporates the biochemical and mechanical stimuli and their interaction that influence endochondral ossification during embryonic growth. The mechanobiochemical framework allows us to probe the effects of molecular events and mechanical loading on development of bone.

\footnotetext{
${ }^{2}$ The results presented in this chapter has been published in: J. J. Vaca-González, M. Moncayo-Donoso, J. M. Guevara, Y. Hata, S. J. Shefelbine, and D. A. GarzónAlvarado, "Mechanobiological modeling of endochondral ossification: an experimental and computational analysis". Biomech. Model. Mechanobiol., vol. 17, no. 3, pp. 853-875, 2018.
} 


\subsection{Introduction}

Long bone formation starts early during embryonic development with the formation of a cartilaginous mold known as anlage by a process called chondrogenesis [94]-[96]. This cartilage mold is ossified through a process known as endochondral ossification, which is a highly regulated mechanism that involves several mechanical and biochemical factors [28], [97]. Long bones experience a loading environment generated by different sources such as the body weight, the restrictions imposed by nearby structures (periosteum, perichondrium, Lacroix ring and adjacent bones) and the tensile influences exerted by muscles [54], [98], [99]. Mechanical stimuli such as compressive loads, shear stress and hydrostatic pressure have influenced the cell dynamics in terms of proliferation, hypertrophy, apoptosis and expression of growth factors (VEGF, TGF $\beta$, PTHrP) [100]-[102]. Mechanical loading of the growth plate is a regulator of bone growth and ossification [54], [103]-[106], and can influence the morphology of the femur [107], [108]. Regarding the biochemical factors, Indian hedgehog (Ihh), parathyroid hormone-related protein (PTHrP) and vascular endothelial factor (VEGF) are considered the main molecules controlling endochondral bone development [21], [28]. Ihh is a member of the hedgehog family expressed during long bone growth by pre-hypertrophic chondrocytes [25]. It is also a master of bone cells differentiation and acts on perichondrial cells to convert them into osteoblasts and form the bone collar [21], [109]. PTHrP is a protein expressed during fetal stage in the periarticular perichondrium at the end of cartilage mold by proliferative chondrocytes [26]. The transition of chondrocytes from proliferative to hypertrophic within the growth plate is primarily regulated through a negative feedback loop between Ihh/PTHrP [27]. PTHrP acts on proliferative chondrocytes to maintain proliferation, and at the same time, it inhibits the production of Ihh. Thus, if the production of PTHrP is sufficiently distant from the proliferative zone of the growth plate, the production of Ihh will start in the pre-hypertrophic zone. Ihh acts on proliferative chondrocytes to promote their proliferation through PTHrP synthesis stimulation [21], [27], [97], [110]. In addition to these two molecules, VEGF has been involved in blood vessel invasion into hypertrophic cartilage and its 
ossification [29]. VEGF is an angiogenic stimulator which is synthesized by hypertrophic chondrocytes that also induce migration and differentiation of osteoblast and osteoclast [28]. VEGF is a specific mitogen for vascular endothelial cells to serve as an important mediator during the endochondral ossification process, especially in the formation of the primary ossification center (POC), due to its ability to regulate neovascularization into hypertrophic cartilage [27], [29], [30]. Once blood vessels are created, they allow the entrance of mesenchymal cells into the ossification front near to the hypertrophic zone of the growth plate. These cells differentiate into osteoblast and engage in osteogenesis [31].

In order to study experimentally, the early stages of long bone formation during embryonic development researchers have been able to observe details about bone development in bovines, equines and humans using techniques such as magnetic resonance imaging (MRI) [111]. Cole et al., showed that in a prenatal stage the diaphysis of the murine femur is completely ossified, and the epiphyses are avascular cartilage tissues (Figure 3-1). Moreover, they elucidate in postnatal development the differences between proximal and distal epiphysis ossification. These findings revealed that the murine femur provides an in vivo model for epiphyseal development, mineralization, physeal resorption and the development of the vascular supply of bone [112].

Mathematical models of the endochondral ossification process have been developed. For instance, the mathematical models implemented have simulated the effect of mechanical loading on the endochondral ossification, examining formation of the secondary center [113], [114], growth plate progression [115], and growth plate zone size [103], [104], and development of bone deformities [116]. Our group has previously used computational models based on the reaction-diffusion equations to explain how biochemical factors affect long bone formation, including the Ihh/PTHrP-negative loop and VEGF stimulator within the growth plate and bone rudiment [28], [105], [117], [118], the spongiosa primary development [104], [119], the growth pattern of the secondary ossification center [104], [117], [120], and the formation of cartilaginous canals in the epiphysis [121]. 


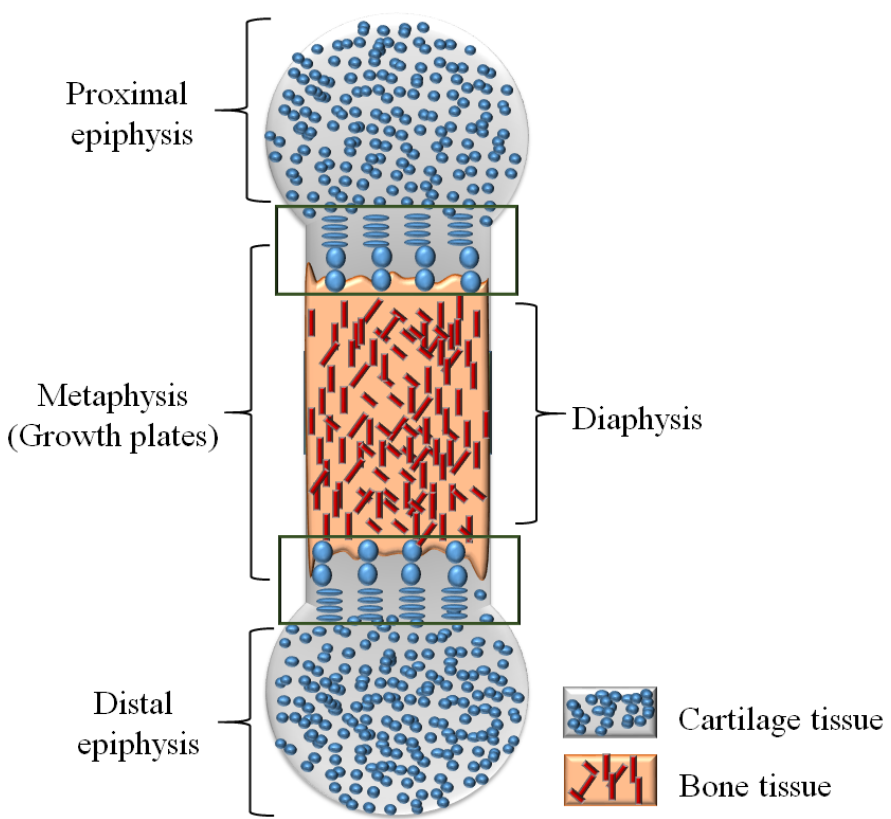

Figure 3-1: Generic long bone parts.

Previous mathematical models of endochondral ossification have studied the mechanical and biochemical factors involved in bone development separately. Moreover, those models have evaluated long bone formation assuming a preexisting bone shape and ossification of the primary center [28], [103], [113], [120], [121]. Here we present a mathematical model that simulates the endochondral ossification process from a pure cartilage anlage to formation (but no ossification) of the epiphyses taking into account biochemical reactions and mechanical loading. The aim of our research was to study experimentally the morphological development of a mice femur in prenatal stage and the implementation of a mechanobiological model to assess computationally the effect of mechanical loads on the behavior of Ihh/PTHrP negative loop and VEGF protein during long bone growth and shape. The mechanobiochemical model considers Ihh, PTHrP and VEGF, the effect of the active (convection) and passive (diffusion) transport on these molecules and the mechanical effect on the growth. Thus, the model could reproduce the shape, internal structures as POC, bone collar, growth fronts and the distribution of the concentration of each molecule that could be corroborated by histological previous 
reports [8], [27], [95]. This mechanobiological model may be useful to simulate effects of altered loads or variations in molecular concentration in order to predict skeletal abnormalities.

\subsection{Materials and methods}

An experimental analysis and a mechanobiological model were implemented to observe and simulate the endochondral ossification process of a femur during embryonic development. The experimental assay consisted of analyzing the morphological changes in the femur of a mice embryo through histologies and imaging, while with the mathematical model it was intended to predict the role of mechanical and biochemical factors during long bone formation.

\subsubsection{Experimental data}

\subsubsection{Tissue samples}

Balb/c mice were obtained from the Basic Science-Faculty Pharmacy Department-and killed at the Biomimetics Laboratory of the Biotechnology Institute at Universidad Nacional of Colombia. Femurs from mice in prenatal stages at 15 (E15), 17 (E17) and 19 (E19) days of age were isolated and fixed in $4 \%$ of formaldehyde. Recommendations from the ethics committee were taken into account for this procedure, following the Article 11 of Resolution No. 008430 of 1993 of the Colombian Ministry of Health. The gestational age of the female mice was measured taking the appearance of the vaginal plug which represent the first day of pregnancy.

\subsubsection{Measurement of length and thickness}

Measurements of length and thickness of the epiphysis were taken from femurs isolated at days 15,17 and 19 of pregnancy. Measurements were taken by triplicate through an image analysis from pictures using the software ImageJ version 1.48. 


\subsubsection{Alcian blue/Alizarin red staining}

The histological evolution of the endochondral ossification process was analyzed with Alcian blue and Alizarin red [122], [123]. Embryos were submerged in a solution of Alcian blue at $0.03 \%$ during 3 days at room temperature. Then, samples were washed with ethanol at 95\% during 6 $\mathrm{h}$ in constant agitation. Later, samples were washed with a solution of $\mathrm{KOH}$ at $2 \%$ in a time period of $12-24 \mathrm{~h}$ at room temperature. Once Alcian blue staining was done, embryos were submerged in a solution of Alizarin red at $0.03 \%$ during $24 \mathrm{~h}$ at room temperature. Then, samples were washed 3 times with glycerol/1\% $\mathrm{KOH}$ with a gradual increase in glycerol concentration (20, 50 and $80 \%)$; each wash had an incubation time of $24 \mathrm{~h}$ at room temperature. Finally, all stained embryos were stored in glycerol at $100 \%$ and were submitted to a transparency process with $\mathrm{KOH}$ before staining.

\subsubsection{Histological and image analysis}

For histological analysis of embryonic stage bones, samples were embedded in paraffin and sectioned at $5 \mu \mathrm{m}$ and observed microscopically. Images from epiphyses, POC and growth plates were taken using a microscope with integrated camera (Nikon TE300, Melville, NY USA).

\subsubsection{Micro-CT}

Micro-CT image acquisition was done after phosphotungstic hematoxylin staining that allows to observe the soft tissue. All samples were scanned using an X-ray microtomography Xskyscan 1172 (Micro Photonics, Allenton, PA, USA), and the acquisition parameters were adjusted to $60 \mathrm{kV}, 124 \mu \mathrm{A}, 180^{\circ}$ with 6 pictures on average and a rotation time of 0.6. The 3D reconstruction was performed using NRECON (Micro Photonics, Allenton, PA, USA). A 10-unit factor signal edition and artifact corrections for a $60 \%$ beam hardening were used. The 3D images were generated using CtVox (Micro Photonics, Allentown, PA, USA). 


\subsubsection{Mathematical model}

\subsubsection{Geometrical model and boundary conditions}

A bi-dimensional domain to represent the anlage with an initial condensation of chondrocytes was performed in this study (Figure 3-2).
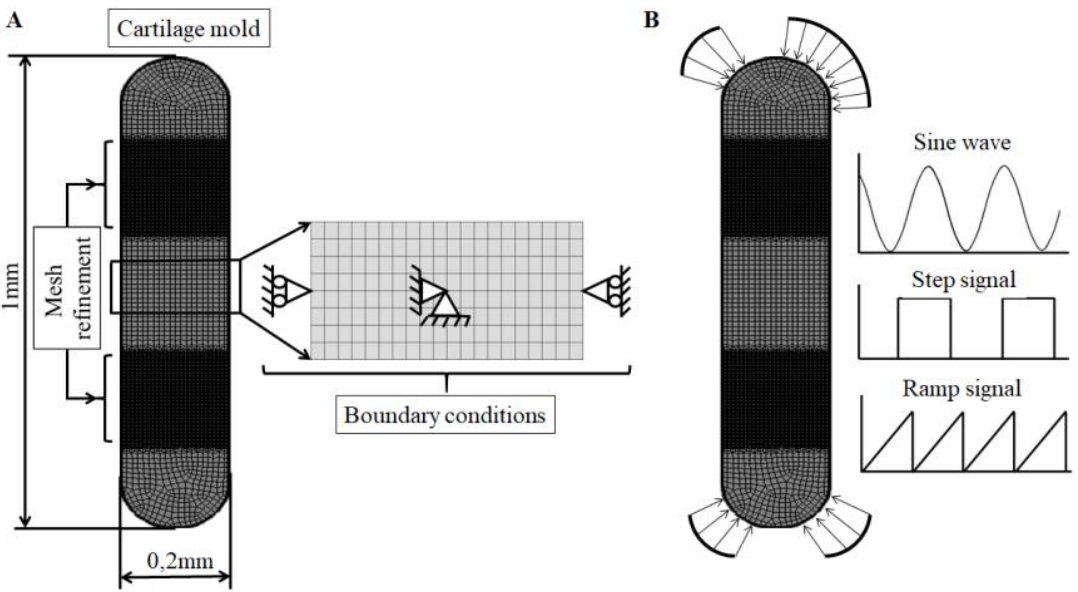

Figure 3-2: Representative scheme of the anlage geometry and boundary conditions.

A) Dimension of the anlage and boundary conditions. B) The loads applied to the mold were distributed in four parts, two loads were applied at the top of the anlage, while two loads were applied at the bottom. Loads were applied as a sine wave, a step and a ramp.

The length $(1 \mathrm{~mm})$ and width $(0.2 \mathrm{~mm})$ of the cartilage mold were obtained from [124]. The 2D domain was meshed using 4-node elements in order to obtain 14,738 nodes and 14,504 elements, respectively. A mesh refinement was done in two specific locations of the anlage in order to better visualize the results when the growth fronts appeared (Figure 3-2A). In this study, the boundary conditions and loads have been assumed in order to simulate the development of a femur. The model was fixed at three central nodes in order to prevent movement in $x$ and $y$ directions. 


\subsubsection{Mechanical factors and material properties}

Cartilage exhibits a biphasic behavior; therefore, it can be analyzed as a poroelastic material [121]. Poroelastic behavior is defined by [125]:

$$
\begin{gathered}
-\nabla \cdot \boldsymbol{\sigma}+\nabla P=0 \\
\frac{\partial}{\partial t}(\nabla \cdot \boldsymbol{u})-\nabla \cdot(k \nabla P)=0
\end{gathered}
$$

Eq. 3-1 is derived from the conservation momentum law. This equation couples a linear elastic term $(-\nabla \cdot \sigma)$ with a term that represents the fluid pressure $(\nabla P)$. Eq. 3-2 is the difference between the dilatation change in a solid matrix $\left(\frac{\partial}{\partial t}(\nabla \cdot u)\right)$ and the mechanical load created by the divergence of the fluid pressure gradient, where $k$ is the permeability of the solid.

Dynamic loads were applied at the top and bottom of the anlage using three different pressure profiles: a sine wave, a step load and release and a ramp load (Figure 3-2B). In order to simulate the endochondral ossification of a femur, two loads were applied at the top of the anlage, representing the force on the greater trochanter and the acetabulum. Two loads were applied at the bottom of the anlage simulating the forces at femoral condyles. In this study, a load of $1 \mathrm{mN} / \mathrm{mm}^{2}$ was assumed based on muscle cross-sectional area and allowable maximum embryonic muscle stress [116], [126]. Frequencies of $0.01,0.1,1$ were selected to apply the dynamic mechanical loading because these are similar to frequencies of muscle contraction in utero [127]. Additionally, a frequency of $10 \mathrm{~Hz}$ was modeled in order to predict the effect of high magnitudes in long bone formation. Simulations were also performed with only the reaction-diffusion equations in order to observe the growth without mechanical loading. The material properties used in this study for each tissue type were poroelastic (Table 3-1). The anlage was meshed using Trelis FEATM (Csimsoft, Utah, USA). 
Table 3-1: Material properties used in finite element model. Cartilage properties are applied to the anlage and the growth fronts, immature bone properties are applied to the POC and mature bone properties are applied to the bone collar [128].

\begin{tabular}{cccc}
\hline & Cartilage & Immature bone & Mature bone \\
\hline $\begin{array}{c}\text { Young's } \\
\text { modulus } \\
(\text { MPa })\end{array}$ & 10 & 1000 & 6000 \\
$\begin{array}{c}\text { Permeability } \\
\left(\mathbf{m}^{\mathbf{4} / N s} \times \mathbf{1 0}^{-14}\right)\end{array}$ & 0.5 & 0.1 & 0.37 \\
Poisson's ratio & 0.167 & 0.3 & 0.3 \\
\hline
\end{tabular}

\subsubsection{Molecular regulatory system}

In this study, it was considered that endochondral ossification process is regulated by three molecules: Ihh, PTHrP and VEGF, the main regulators of bone growth [26], [28], [102], [114], [118], [120], [121]. Other growth factors such as FGF, BMPs and WNTs are involved in bone development, but have not yet been shown to have a clear role in endochondral ossification and were not considered in this study. The time variation of the concentration of the negative feedback loop between Ihh/PTHrP and the concentration of VEGF was modeled by a set of reaction-diffusion equations that can be written in general form as:

$$
\frac{\partial S_{\mathrm{i}}}{\partial t}+\nabla \cdot\left(\boldsymbol{v}_{\boldsymbol{T}} S_{\mathrm{i}}\right)=D_{\mathrm{i}} \nabla^{2} S_{\mathrm{i}}+f\left(S_{\mathrm{i}}, t\right)
$$

where $S_{\mathrm{i}}$ is the concentration of the interacting molecule, $\boldsymbol{v}_{\boldsymbol{T}}$ is the total velocity vector, $D_{\mathrm{i}}$ is the diffusion coefficient of the molecule and $f\left(S_{\mathrm{i}}, t\right)$ is the production rate of the molecule $i$. The reaction term $f$ includes the interactions between molecular factors and their synthesis by the cells. In Eq. 3-3 the total velocity vector $\left(\boldsymbol{v}_{\boldsymbol{T}}\right)$ has two components which are the fluid velocity and growth tissue velocity. The first one is given by the fluid in the poroelastic model $\left(\boldsymbol{v}_{\boldsymbol{f}}=-\boldsymbol{k} \nabla \mathrm{p}\right)$ and 
the second one is given by the growth tensor $\left(\boldsymbol{v}_{\boldsymbol{g}}\right)$. Accordingly, the total velocity vector can be written as follows:

$$
v_{T}=v_{f}+v_{g}
$$

Replacing $\boldsymbol{v}_{\boldsymbol{T}}$ in Eq. 3-4, the term $\nabla \cdot\left(\boldsymbol{v}_{\boldsymbol{T}} S_{\mathrm{i}}\right)$ may be written as:

$$
\begin{gathered}
\nabla \cdot\left(\boldsymbol{v}_{\boldsymbol{T}} S_{\mathrm{i}}\right)=\boldsymbol{v}_{\boldsymbol{f}} \cdot \nabla\left(S_{\mathrm{i}}\right)+\boldsymbol{v}_{\boldsymbol{g}} \cdot \nabla\left(S_{\mathrm{i}}\right)+\left(S_{\mathrm{i}}\right) \nabla \cdot \boldsymbol{v}_{\boldsymbol{f}} \\
+\left(S_{\mathrm{i}}\right) \nabla \cdot \boldsymbol{v}_{\boldsymbol{g}}
\end{gathered}
$$

Simplifying Eq. 3-5 and considering the incompressibility of the fluid in the tissue, the term $\nabla \circ v_{f}=0$. Additionally, since the model is being developed in a Lagrangian framework (material description), it is possible to define the total derivative over time of a concentration $S_{\mathrm{i}}$ as:

$$
\frac{D S_{i}}{D t}=\frac{\partial S_{\mathrm{i}}}{\partial t}+\boldsymbol{v}_{\boldsymbol{g}} \cdot \nabla\left(S_{\mathrm{i}}\right)
$$

Resulting from Eq. 3-6, the left hand side of Eq. 3-3 can be written as:

$$
\frac{\partial S_{\mathrm{i}}}{\partial t}+\nabla \cdot\left(\boldsymbol{v}_{T} S_{\mathrm{i}}\right)=\frac{D S_{i}}{D t}+\boldsymbol{v}_{\boldsymbol{f}} \cdot \nabla S_{\mathrm{i}}+S_{\mathrm{i}} \nabla \cdot \boldsymbol{v}_{\boldsymbol{g}}
$$

Where the term $\frac{D S_{i}}{D t}$ is the total variation in time of the molecule concentration of a material point in the domain, the term $\left(\boldsymbol{v}_{\boldsymbol{f}} \cdot \nabla S_{\mathrm{i}}\right)$ represents the convection or the active transport of the molecule $S_{i}$ due to the fluid velocity $\boldsymbol{v}_{\boldsymbol{f}}$, and the term $\left(S_{\mathrm{i}} \nabla \cdot \boldsymbol{v}_{\boldsymbol{g}}\right)$ represents the concentration change due to dilatation (growth) of the domain.

The model was performed in two different scales of time. The first-time scale was for growth in days, while the second one was for the applied loads in seconds. In order to observe how the velocity of fluid flows in 
the anlage affects the growth and diffusion of the molecules during the endochondral ossification process, the fluid velocity $\boldsymbol{v}_{f}$ was calculated as average velocity (Eq. 3-8) or the maximum velocity (Eq. 3-9) in each node of the domain.

$$
\begin{gathered}
\overline{\boldsymbol{v}_{\boldsymbol{f}}}=\frac{1}{n} \sum_{\substack{i=1 \\
\boldsymbol{m a x}_{\boldsymbol{f}}}}^{n} \boldsymbol{v}_{\boldsymbol{i}} \\
\boldsymbol{v}_{\boldsymbol{i} \mathbf{1 , n} \boldsymbol{n}}\left\{\boldsymbol{v}_{\boldsymbol{i}}\right\} t
\end{gathered}
$$

Where $\overline{\boldsymbol{v}_{\boldsymbol{f}}}$ represents the average velocity in each node $i, \boldsymbol{v}_{\text {fmax }}$ is the maximum velocity in each element, $n$ represents the steps in a time $t$ and $\boldsymbol{v}_{\boldsymbol{i}}$ is the velocity in each node.

The reaction-diffusion system is based on an activator (PTHrP) which diffuses quickly and the inhibitor (Ihh) that diffuses slowly. In the activator-inhibitor system proposed here, PTHrP inhibits Ihh synthesis, while Ihh promotes PTHrP expression [54], [129]. The differential equations that model the reaction-diffusion loop between Ihh/PTHrP are:

$$
\begin{aligned}
& \frac{\partial S_{\mathrm{PTHrP}}}{\partial t}+\nabla \cdot\left(\boldsymbol{v}_{\boldsymbol{T}} S_{\mathrm{PTHrP}}\right) \\
&= D_{\mathrm{PTHrP}} \nabla^{2} S_{\mathrm{PTHrP}}+f\left(S_{\mathrm{PTHrP}}, S_{\mathrm{Ihh}}\right) \\
& \frac{\partial S_{\mathrm{Ihh}}}{\partial t}+\nabla \cdot\left(\boldsymbol{v}_{\boldsymbol{T}} S_{\mathrm{Ihh}}\right) \\
&=D_{\mathrm{Ihh}} \nabla^{2} S_{\mathrm{Ihh}}+g\left(S_{\mathrm{PTHrP}}, S_{\mathrm{Ihh}}\right)
\end{aligned}
$$

Where $S_{\mathrm{PTHrP}}$ and $S_{\text {Ihh }}$ are the concentration of Ihh or PTHrP, $D_{\mathrm{PTHrP}}$ and $D_{\text {Ihh }}$ are the diffusion coefficients of Ihh or PTHrP, and $f\left(S_{\mathrm{PTHrP}}, S_{\mathrm{Ihh}}\right)$ and $g\left(S_{\mathrm{PTHrP}}, S_{\mathrm{Ihh}}\right)$ are the reaction terms between the two molecules. In this model, it was assumed that Ihh is synthetized by proliferative chondrocytes and PTHrP is synthetized by prehypertrophic chondrocytes. Therefore, the production of PTHrP and Ihh depends on chondrocytes concentration of each [117]. The reaction terms for Ihh and PTHrP are written as follows: 


$$
\begin{array}{cc}
f\left(S_{P T H r P}, S_{I h h}\right)=\gamma C_{p}\left(\alpha_{1}-S_{P T H r P}+S_{P T H r P}^{2} S_{I h h}\right) & \text { Eq. 3-12 } \\
g\left(S_{P T H r P}, S_{I h h}\right)=\gamma C_{p}\left(\alpha_{2}-S_{P T H r P}^{2} S_{I h h}\right) & \text { Eq. 3-13 }
\end{array}
$$

Where $\gamma$ is rate value for the reaction term, $C_{p}$ is the concentration of proliferative chondrocytes, $\alpha_{1}$ and $\alpha_{2}$ are constant source terms that quantify the production of each molecular factor by cells, and the term $S_{P T H r P}^{2} S_{\text {Ihh }}$ represents the nonlinear production of PTHrP by the presence of Ihh, and the degradation of Ihh by the presence of PTHrP [130].

Using the definition of equation (Eq. 3-7), the left hand side of Eq. 3-10 and Eq. 3-11 may be written as:

$$
\begin{gathered}
\frac{D S_{\mathrm{PTHrP}}}{D t}+\boldsymbol{v}_{\boldsymbol{f}} \cdot \nabla S_{\mathrm{PTHrP}}+S_{\mathrm{PTHrP}} \nabla \cdot \boldsymbol{v}_{\boldsymbol{g}} \\
=D_{\mathrm{PTHrP}} \nabla^{2} S_{\mathrm{PTHrP}}+f\left(S_{\mathrm{PTHrP}}, S_{\mathrm{Ihh}}\right) \\
\begin{aligned}
\frac{D S_{\mathrm{Ihh}}}{D t}+\boldsymbol{v}_{\boldsymbol{f}} \cdot & \nabla S_{\mathrm{Ihh}}+S_{\mathrm{Ihh}} \nabla \cdot \boldsymbol{v}_{\boldsymbol{g}} \\
= & D_{\mathrm{Ihh}} \nabla^{2} S_{\mathrm{Ihh}}+g\left(S_{\mathrm{PTHrP}}, S_{\mathrm{Ihh}}\right)
\end{aligned}
\end{gathered}
$$

We analyzed the sensitivity of the reaction-diffusion system by examining what happens when Ihh and PTHrP are not expressed or are overexpressed. Eq. 3-14 and Eq. 3-15 establish a nonlinear interaction between the activator PTHrP and an inhibitor Ihh. Patterns, called Turing patterns, can emerge when certain requirements are fulfilled [131]. The reaction-diffusion system must satisfy Eq. 3-16, Eq. 3-17 and Eq. 3-18 in order to guarantee the stability and pattern formation of the system.

$$
\begin{gathered}
\frac{\boldsymbol{\partial f}}{\partial \boldsymbol{S}_{\boldsymbol{u}}}+\frac{\boldsymbol{\partial g}}{\boldsymbol{\partial \boldsymbol { S } _ { \boldsymbol { v } }}}<\mathbf{0} \\
\frac{\partial f}{\partial S_{u}} \cdot \frac{\partial g}{\partial S_{v}}-\frac{\partial f}{\partial S_{v}} \cdot \frac{\partial g}{\partial S_{u}}>0
\end{gathered}
$$




$$
\begin{gathered}
D_{u} \frac{\partial g}{\partial S_{v}}+D_{v} \frac{\partial f}{\partial S_{u}}>2 \sqrt{D_{u} D_{v}} \sqrt{\frac{\partial f}{\partial S_{u}} \cdot \frac{\partial g}{\partial S_{v}}-\frac{\partial f}{\partial S_{v}} \cdot \frac{\partial g}{\partial S_{u}}} \quad \text { Eq. 3-18 } \\
>0
\end{gathered}
$$

Where the functions are evaluated in the steady state solution $\left(S_{u}^{*}, S_{v}^{*}\right)$, which is given by $f\left(S_{u}^{*}, S_{v}^{*}\right)=0$ and $g\left(S_{u}^{*}, S_{v}^{*}\right)=0$. If the parameters of Eq. 3-14 and Eq. 3-15 satisfy the inequalities Eq. 3-16, Eq. 3-17, and Eq. 3-18, then the equations are in the Turing space parameters.

\subsubsection{VEGF diffusion}

VEGF promotes the vascularization of the hypertrophic zone of the growth fronts in order to form the bone [29]. The diffusion of VEGF from hypertrophic chondrocytes can be written similarly to equation (3) as follows:

$$
\begin{aligned}
\frac{D S_{V E G F}}{D t}+\boldsymbol{v}_{\boldsymbol{f}} \cdot \nabla S_{V E G F}+S_{V E G F} \nabla \cdot \boldsymbol{v}_{\boldsymbol{g}} \\
=D_{V E G F} \nabla^{2} S_{V E G F}+\underbrace{r S_{V E G F} C_{H}\left(1-\frac{S_{V E G F}}{P_{V E G F}}\right)}_{\text {Logistic growth }}+\chi_{V E G F} C_{H}
\end{aligned}
$$

Where $S_{\mathrm{VEGF}}$ represents the concentration of VEGF in the tissue and $D_{\mathrm{VEGF}}$ is the diffusion coefficient of the molecule. Eq. 3-19 has a term that represents the logistic growth of a given population. In this case, this population is represented by $P_{V E G F}$ which indicates the VEGF molecule widespread in the domain, $C_{H}$ is the concentration of hypertrophic chondrocytes, $r$ is the exponential growth rate of VEGF and $\chi_{V E G F}$ represents the constant production of VEGF by the hypertrophic chondrocytes.

\subsubsection{Growth rate}

Growth and morphogenesis were controlled by biochemical and mechanical factors. It was assumed that the mechanical loading has little influence early in development (before joints form and muscles contacts), and the growth rate of the anlage is a process purely 
biochemical that only depends on Ihh and PTHrP. The equation that represents early growth is written in a general form as:

$$
\begin{gathered}
\boldsymbol{d}_{\boldsymbol{i}, \boldsymbol{j}}^{\text {growth }}(x, y) \\
=\left\{\begin{array}{c}
\text { for } d_{i}+\alpha_{\text {PTHrP }}^{i} S_{P T H r P}+\alpha_{\nabla P T H r P}^{i}\left(\nabla S_{P T H r P}\right)_{i}+\beta_{i h h}^{i} S_{i h h} \\
+\beta_{\nabla i h h}^{i}\left(\nabla S_{i h h}\right)_{i} \\
\text { else }=0
\end{array}\right.
\end{gathered}
$$

Where $\boldsymbol{d}_{\boldsymbol{i}, \boldsymbol{j}}^{\text {growth }}(\boldsymbol{x}, \boldsymbol{y})$ is the strain tensor of the growth rate, $d_{i}$ is a constant related with tissue elongation, $S_{P T H r P}$ and $S_{i h h}$ are the concentrations of the molecules PTHrP and Ihh and $\alpha_{\text {PTHrP }}^{i}$ and $\beta_{i h h}^{i}$ are constants that indicate the influence of the concentration of each molecule on the isometric growth, $\alpha_{\nabla P T H r P}^{i}$ and $\beta_{\nabla i h h}^{i}$ are constants which determine how much the gradient of the concentration of each molecule influences on directional growth.

At later stages of development, tissue growth depends on the molecule concentrations, and on the dynamic hydrostatic stress. The equation that represents later growth is written in general form as:

$$
\boldsymbol{d}_{i, j}^{\text {growth }}(x, y)=\left\{\begin{array}{cc}
\text { for } & d_{i}+\alpha_{P T H r P}^{i} S_{P T H r P}+\alpha_{\nabla P T H r P}^{i}\left(\nabla S_{P T H r P}\right)_{i} \\
i=j & +\left(\left(\varphi_{H}^{D} \sigma_{H}^{D}\right) * S_{P T H r P}\right) \\
\text { else }=0
\end{array}\right.
$$

Where $\varphi_{H}^{D}$ is the growth constant in the dynamic state, $\sigma_{H}^{D}$ is the dynamic hydrostatic stress obtained from the poroelastic model. The hydrostatic stresses obtained from the simulations were the result of the sum between pore pressure and the trace of the matrix stress. Chondrocytes in resting and proliferative zones of the growth front can proliferate to promote growth of the femoral head. This volumetric expansion of the tissue is due to a constant increase in cell concentration, which is controlled by the molecular synthesis of PTHrP and the compressive hydrostatic stresses, respectively. For this reason, in equation Eq. 3-21 the PTHrP concentration and the hydrostatic stresses are multiplied since to a high concentration of these two factors, 
the growth of the tissue will be higher in comparison with regions where the PTHrP or hydrostatic stresses are lowest.

\subsubsection{Bone collar formation}

The bone collar is a tissue of the perichondrium which is the initial ossification of the cartilage mold [21]. This structure is formed during endochondral ossification by a remaining concentration of Ihh in the perichondrium that differentiates perichondrial cells into osteoblasts [109]. Taking this into account, the Eq. 3-22 is stablished to form the bone collar, where $S_{P T H r P}^{T H r}$ represents a threshold value of the concentration of Ihh in the perichondrium.

$$
S_{I h h}>S_{I h h}^{T h r}
$$

\subsubsection{Bone elongation and ossification}

An algorithm to model cartilage elongation and ossification was developed. First, the algorithm identifies the stratified columns of the proliferative zone of the growth front (Figure 3-3 left side). Then, the chondrocytes begin to hypertrophy in order to start the vascularization at the ossification front (Figure 3-3 center). Finally, the hypertrophic chondrocytes direct the formation of mineralized matrix, attract blood vessels, and undergo apoptosis (Figure 3-3 right side). Here, it is crucial to take into account the time that a chondrocyte takes in forming the different growth plate zones and mineralize the tissue. According to [16], this time is $24 \mathrm{~h}$; therefore, in the present model the time that a cell takes to form the growth fronts, then mineralize the tissue and dye by apoptosis is approximately $20 \mathrm{~h}$.

The magnitude and direction of ossification front (cartilage turning into bone) was determined by the following equation:

$$
\boldsymbol{d}_{i, j}^{\text {growth }}(x, y)=\frac{\left(\mu_{x, y} S_{P T H r P}^{T h r}\right)^{n}}{\left(S_{P T H r P}^{T h r}\right)^{n}+\left(S_{P T H r P}\right)^{n}} * \frac{\left(\operatorname{grad} S_{V E G F}\right)}{\left\|\operatorname{grad} S_{V E G F}\right\|}
$$


Where $S_{P T H r P}^{T h r}$ is represents a threshold value of the concentration of PTHrP in the hypertrophic zone of the growth plate, $\mu_{x, y}$ is a growth constant and $\operatorname{grad} S_{V E G F}$ is the gradient of the concentration of VEGF in the ossification front. The parameters of the equations described above are listed in Appendix A.
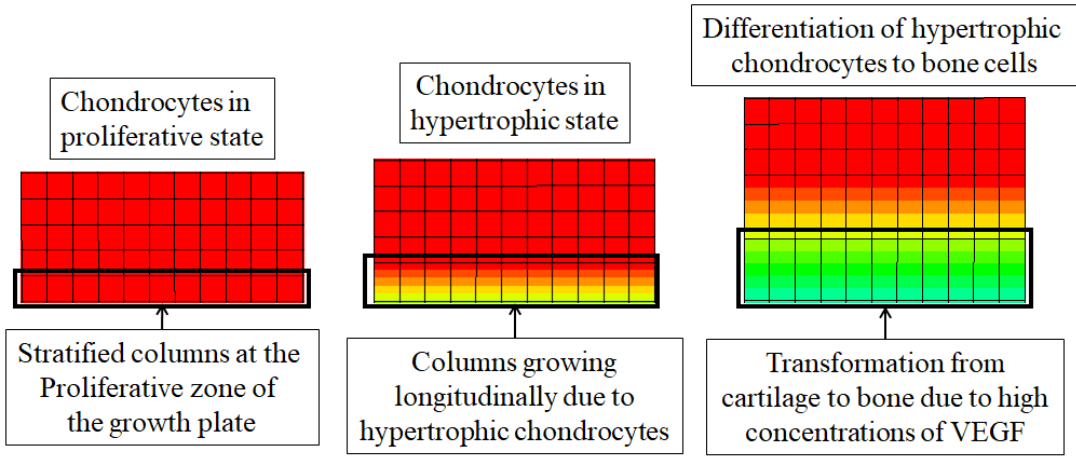

Figure 3-3. Representative scheme of bone elongation and ossification on the growth plate zone.

In this model each column in the proliferative zone of the growth plate has the possibility to grow independently.

\subsubsection{Model Implementation}

A multiscale approach in time was performed to solve the mathematical model (Figure 3-4) because diffusion of Ihh, PTHrP and VEGF occurs over days, while the cyclic loading is applied in seconds. Ihh and PTHrP are first randomly distributed as initial condition around a steady state by the chondrocytes within the anlage. When the loads are applied, the fluid velocities and dynamic hydrostatic stresses are stored for each node and included into the equations that govern cartilage elongation and mineralization. This process is repeated until the levels of Ihh achieve a threshold value in the center of the cartilage mold $\left(S_{I h h}^{T h r}=\right.$ 0.85 ). Once the Ihh levels are high in the diaphysis (between 0.93 and 0.975), the chondrocytes hypertrophy allowing blood vessels invasion to form the POC. The remainder Ihh concentrations at the edge of the diaphysis induce differentiation of perichondrial cells into osteoblasts to form the bone collar. 


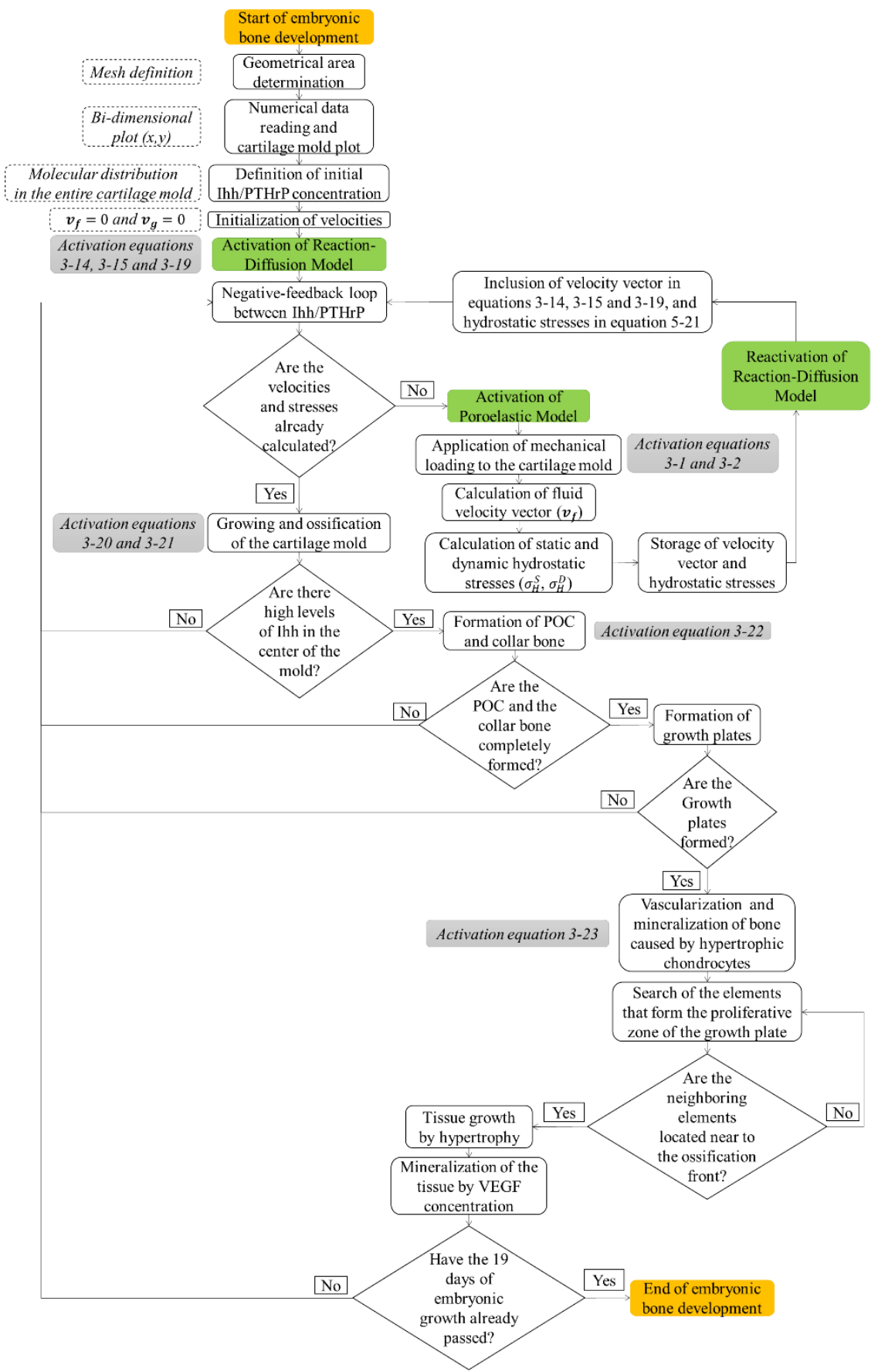

Figure 3-4: Representative scheme of the multiscale computational modeling. 
When the POC is completely ossified, the chondrocytes located in the metaphysis are arranged in three different zones (resting, proliferative and hypertrophic) forming the growth fronts. This process is repeated until the epiphyseal plates are formed. When the growth fronts are created, the hypertrophic chondrocytes located at the ossification front begin the mineralization of the tissue. To mineralize the tissue, the algorithm seeks the columns that form the proliferative zone of the growth fronts and make that the chondrocytes enter in hypertrophic state, causing elongation, angiogenesis and ossification of the bone. This final process is repeated until it has reached the 19 days of embryonic gestation (Figure 3-3).

The equations previously described were implemented and numerically solved in a finite element framework to simulate the endochondral ossification process. The computational simulation was performed in an incremental-iterative scheme which allows to compute the evolution of the negative-feedback loop between Ihh/PTHrP and the diffusion of VEGF molecule, while the mechanical loads are been applied. The mechanobiological problem was solved in ABAQUS 6.5.1 by means of a UEL subroutine developed in FORTRAN 90 (Formula Translating System, New York, USA), and the results were visualized in TECPLOT 360 (Tecplot Inc. Bellevue).

\subsection{Results}

\subsubsection{Femur Morphogenesis}

Experimental results indicate that femur increased in length by $2.24 \mathrm{~mm}$ and in thickness by $0.3 \mathrm{~mm}$ from E15 to E19 (Figure 3-5). Morphologically, the bony rudiment at E15 presents the beginning of a femoral head, the greater trochanter and the condyles (Figure 3-6A). At this stage, the micro-CT allows to observe the presence of the POC and the growth fronts. At E17 the femur increased in size, especially proximally (Figure 3-6B). In addition, the femoral head starts to develop allowing the formation of the femoral neck. There is histologic evidence of a slightly reddish staining in the diaphysis that indicates the calcium deposition due to the presence of the POC. 
A

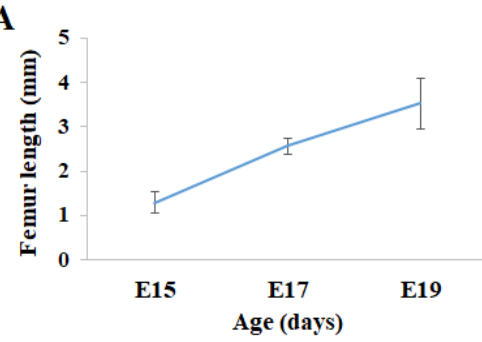

B

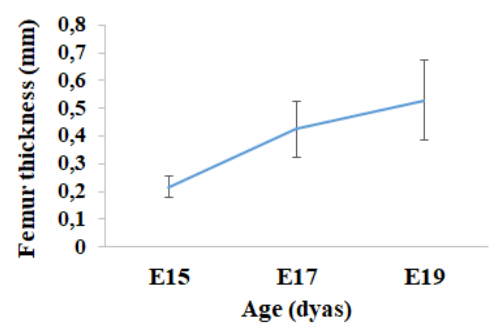

Figure 3-5. Length and thickness curves of a Balb/c mice femur ( $n=3 /$ time point).

A) Longitudinal growth of femur in embryonic stage. B) Growth in thickness of the bone in embryonic stage.

Additionally, the histologies showed chondrocytes forming the different zones of the growth front, where round chondrocytes randomly distributed form the reserve zone, oval chondrocytes arranged into columns form the proliferative zone and big round chondrocytes form the hypertrophic zone. Close to birth, the embryo at E19 presents a femur in which the diaphysis is completely ossified, and the femoral head and greater trochanter are more defined (Figure 3-6C). In addition, at this stage (E19) it is evidenced the transition zone from proliferative to hypertrophic chondrocytes.

Considering that experimental analysis was performed from $15 \mathrm{E}$ to $19 \mathrm{E}$, in this study there is no evidence about the anlage formation and its evolution before 15E. However, the computational model simulated formation from (E1) to the final embryonic stage (E19) (Appendix B). The results obtained from the simulations were comparable to the experimental data, with the appearance of the POC, bone collar and the growth fronts and the shape of the femoral head and greater trochanter (Figure 3-7). The model that best predicted the experimental shape, was for frequency of $0.01 \mathrm{~Hz}$ and a step signal (Figure 3-7A). Bone length and thickness varied slightly between simulations and with experimental measures (Appendix C). Bone morphology changed when the mechanical load was not applied, with no formation of the femoral head or trochanters (Figure 3-7B). 


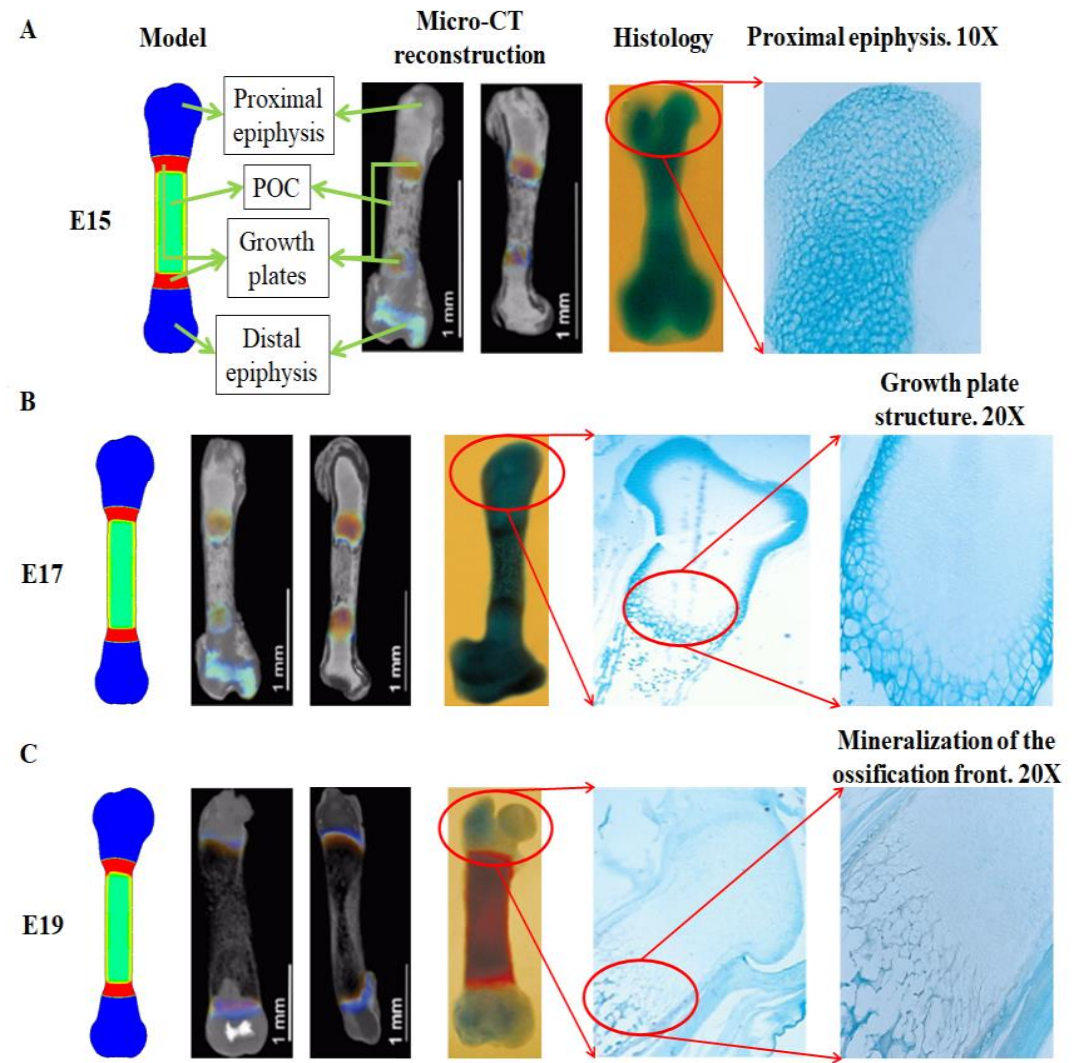

Figure 3-6. Comparison between the computational model and the experimental data.

The model shown in the left side of the figure correspond to the simulation performed with a frequency of $0.01 \mathrm{~Hz}$ and a step signal. A)

Beginning of bone development and the apparition of the internal structures at E15. B) A POC and growth plates completely formed at E17. C) Formation of the femoral head, the trochanter and he condyles at E19.

\subsubsection{Biochemical Regulation}

The biochemical synthesis of Ihh, PTHrP and VEGF for E19 is shown in Figure 3-8. 
A 15 days of embryonic stage $0,01 \mathrm{~Hz} \quad 0,1 \mathrm{~Hz} \quad 1 \mathrm{~Hz} \quad 10 \mathrm{~Hz}$
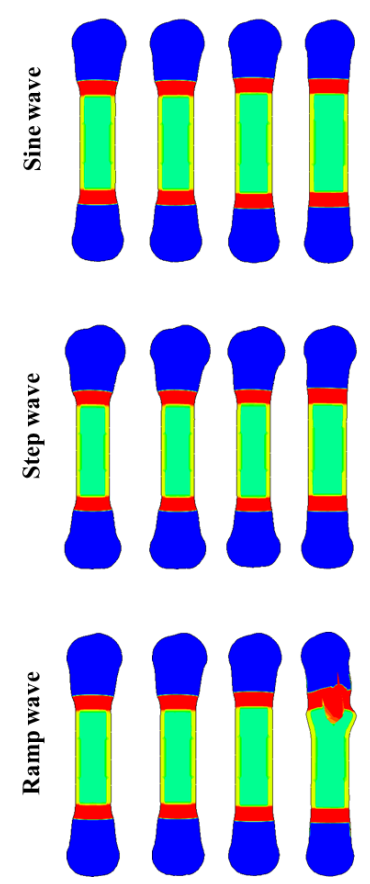

B

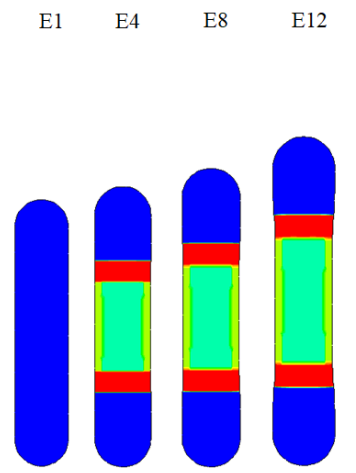

$\mathbf{1 7}$ days of embryonic stage $0,01 \mathrm{~Hz} \quad 0,1 \mathrm{~Hz} \quad 1 \mathrm{~Hz} \quad 10 \mathrm{~Hz}$
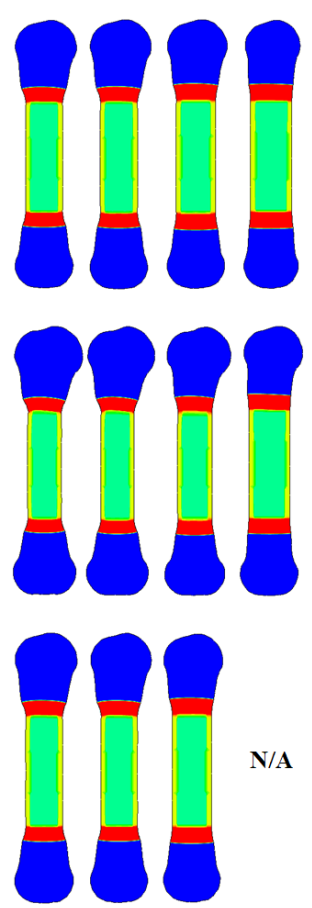

N/A

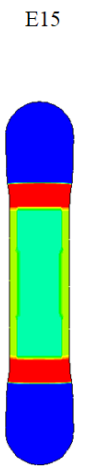

19 days of embryonic stage
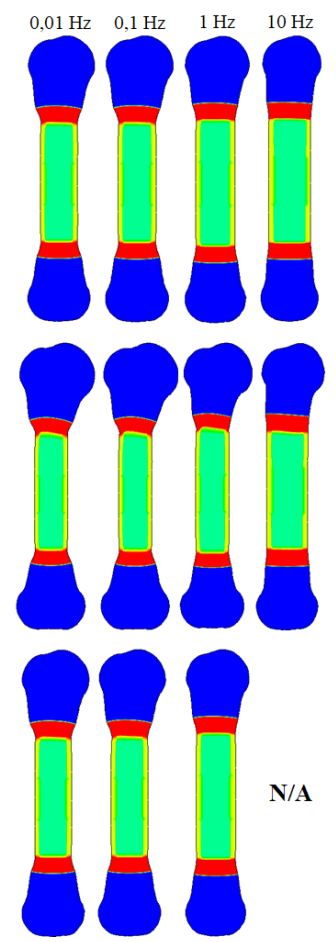

$\mathbf{N} / \mathbf{A}$

Figure 3-7. Bone morphology with and without mechanical load.

A) Changes of the growth and thickness of the bone during embryonic development (E15, E17 and E19) with different frequencies and signals. The acronym N/A means that there is no information about

these simulations since the modelling at these stages was not completed due to computational calculations errors. B) Simulated morphogenesis with no mechanical loading. 
High concentrations of Ihh developed in the growth fronts (Figure 3-8A), while high PTHrP concentrations were found in the epiphysis (Figure 3-8B). VEGF was produced in the POC and extended to the ossification front (Figure 3-8C). The production and diffusion of Ihh, PTHrP and VEGF for the cases studied in this research are showed in Appendix D. Inhibition or overexpression of PTHrP and Ihh resulted in no growth (Data not shown).
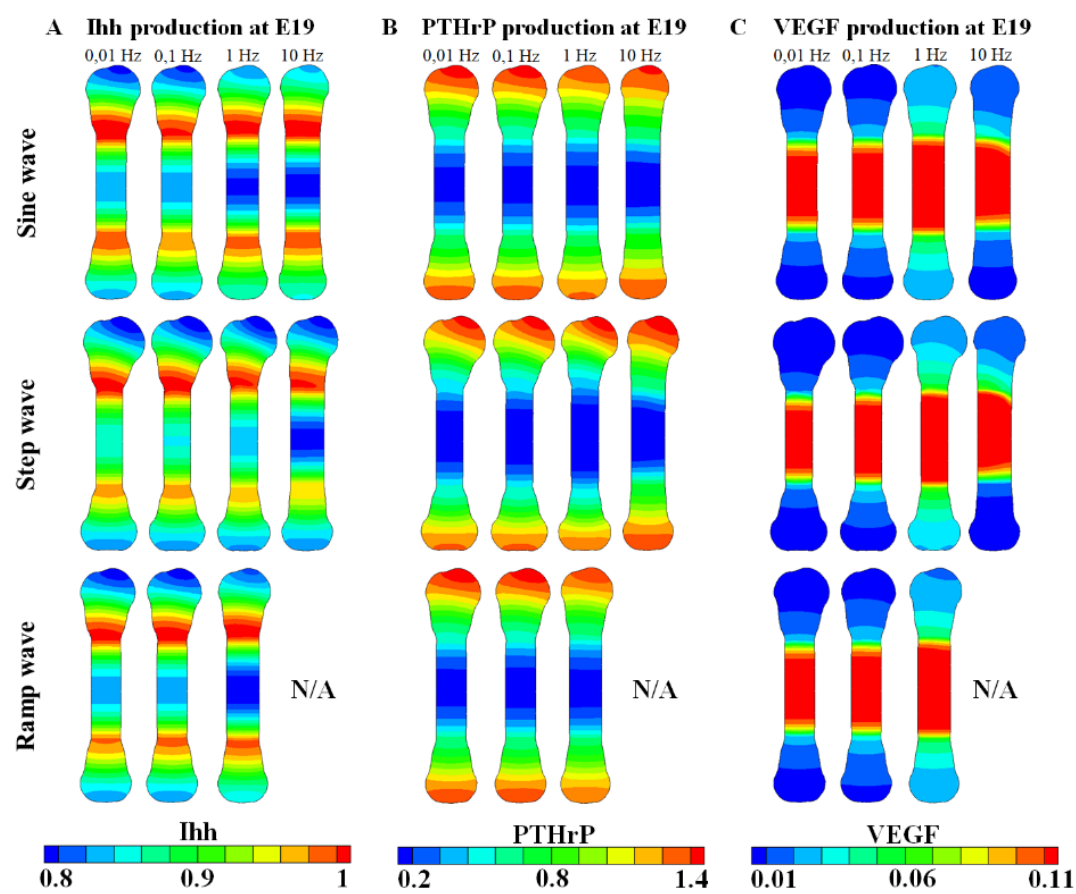

Figure 3-8. Representative scheme of molecular concentrations at E19.

A) Ihh concentrations. B) PTHrP concentrations. C) VEGF concentrations. The acronym N/A means that there is no information about these simulations due to computational modelling at these stages was not completed.

\subsubsection{Hydrostatic Stress and Fluid Velocity Distribution}

Hydrostatic stress and fluid velocity distribution changed according to the loading frequencies applied (Figure 3-9). 

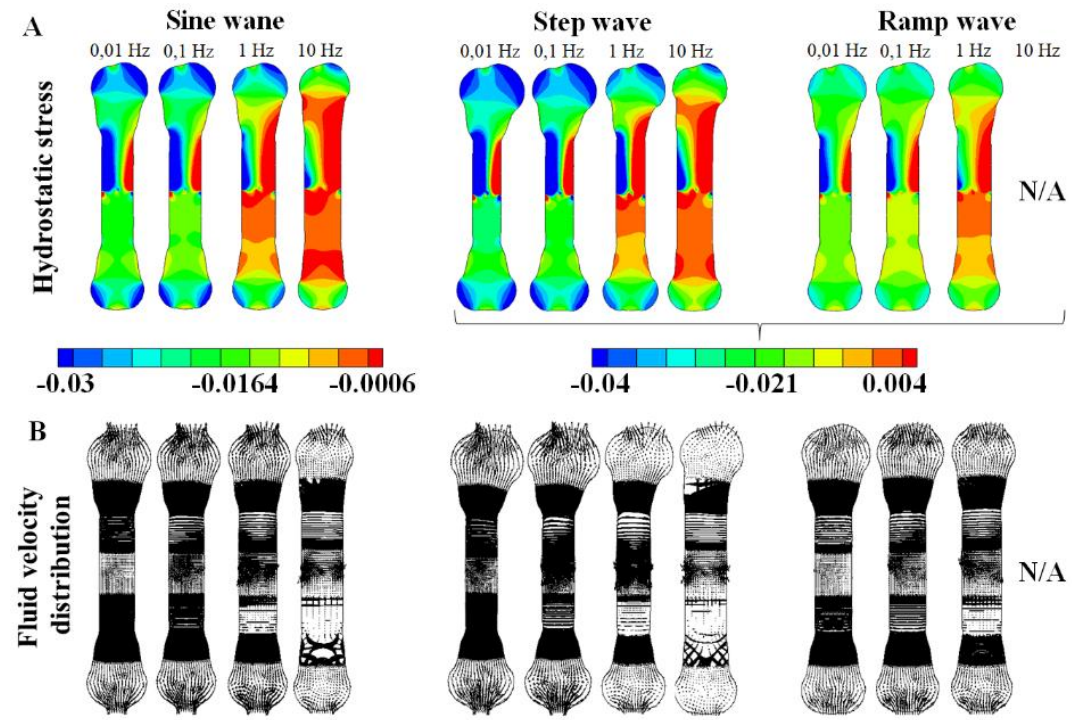

C

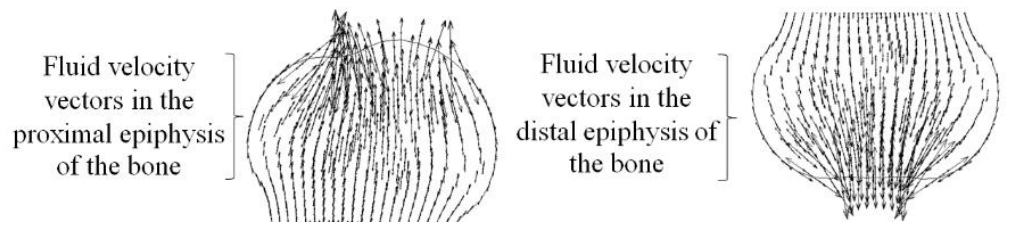

Figure 3-9. Hydrostatic stress and fluid velocity distributions within the cartilage at E19.

A) Hydrostatic stress distribution. B) Fluid velocity distribution. C) Velocity vector in the distal and proximal epiphysis of the bone when a mechanical load was applied using a frequency of $0.01 \mathrm{~Hz}$ and a step signal.

High hydrostatic compressive stresses are located at the epiphysis of the bone near the loads; low hydrostatic stresses are found in the diaphysis and at the growth front. Hydrostatic stress decreased with frequencies (Figure 3-9A). Figure 3-9B shows how the fluid velocities $\left(\boldsymbol{v}_{\boldsymbol{f}}\right)$ are distributed within the bone when a mechanical load is applied, while in Figure 3-9C it is possible to observe in detail the direction in which the fluid comes out from the proximal and distal epiphysis when a mechanical load is applied. 


\subsection{Discussion}

Using the computational model, it was possible to observe minor bone morphology changes due to the frequencies and the type of signals used to apply the load. For instance, the epiphysis was less prominent when the load was applied using a sine and a ramp wave than with step function. This behavior is because hydrostatic stress is higher under step load, because the fluid has less time to flow out compared to a gradual load. The frequency moderately affects bone formation. For instance, with low frequencies $(0.01,0.1$ and $1 \mathrm{~Hz})$ the bone acquired a shape similar to a real femur and the length and size of the epiphysis and diaphysis tend to be equal for the stages assessed experimentally (Figure 3-7). This result may be compared with the research carried out by [116], in which a load applied at $1 \mathrm{~Hz}$ result in prenatal joint shape development. These results suggest that mechanical loading applied with low-frequencies induce mechanotransduction in early stages of development; therefore, mechanoreceptors in cell membrane activate the adequate signaling pathway to express the characteristic molecules involved in osteogenesis. With high frequencies $(10 \mathrm{~Hz})$ the bone loses its ideal morphology and in the majority of cases the endochondral ossification process is interrupted, and the bone does not continue growing (Appendix B). The shape acquired by the bone was highly dependent on the location of the load. In this study the anlage was mechanically loaded during endochondral ossification process, but this behavior does not occur in vivo as the bone, during its embryonic development, may experiment changes in the supported load. Therefore, future work will improve the model so that it can apply the mechanical loads at different intervals of time during the day.

The model developed simulates correctly the reaction-diffusion between the molecules, since the results are in accordance with the observed by other authors such as Provot et al., Ballock et al., and Kronenberg, in which they demonstrate that Ihh induces PTHrP expression at the articular region of the bone and PTHrP in turn represses Ihh expression generating a negative feedback loop. They also state that PTHrP and Ihh are positive modulators of proliferation and negatively affect maturation. It means that the effect of Ihh on 
maturation is exclusively PTHrP dependent, while Ihh induces proliferation independently of PTHrP [16], [21], [27]. The correct expression of Ihh and PTHrP are related with a normal growth of long bones; nevertheless, a disruption in the secretion of one of these molecules can result in pathologies such as acrocapitofemoral dysplasia, which is associated with premature closure of the growth plates, Blomstrand dysplasia, which may cause death in the uterus due to an advanced endochondral maturation, and short stature due to a delay in hypertrophic chondrocyte differentiation [132]. Several studies have demonstrated that a mutation of PTHrP or Ihh leads to a decrease in trabecular bone formation in the POC and a delay in angiogenesis of the early cartilage model [133], alterations in the temporal and spatial sequence of epiphyseal cartilage development [134], reduction in the size of the growth plates of long bones [135], and shortness of absent middle phalanges in digits [136]. In our model, VEGF diffusion did not occur when a mutation of Ihh was done, as this molecule depends on the number of early or late hypertrophic cells [28]-[30].

In addition, the poroelastic formulation implemented in this study demonstrated how the velocity of fluid flow through the tissue affects the molecular diffusion, and therefore the normal bone growth. The convective flow due to mechanical loading applied with low frequencies $\left(0.01,0.1\right.$ and $1 \mathrm{~Hz}$ ) and $1 \mathrm{~N} / \mathrm{mm}^{2}$ of mechanical load do not affect the molecular concentrations of Ihh/PTHrP and VEGF because the velocities at which the fluid passes through the tissues are very small (See Appendix D). Nevertheless, the molecular gradients are altered and the bone present abnormalities during longitudinal growth when high magnitude loads $\left(1,10\right.$ or $\left.100 \mathrm{~N} / \mathrm{mm}^{2}\right)$ are applied (See Appendix E). This finding indicates that high mechanical loads influence the fluid flow and disrupt the biochemical patterning. This behavior generates a downregulation of the biochemical regulation, which leads to an overexpression of the molecules causing anomalies during bone development.

Endochondral ossification is a complex process that is regulated by specific molecules and mechanical stimuli such as tension, compression, shear and hydrostatic pressures [99]-[102], [105], [106]. 
The model presented here, to our knowledge, is the first computational approach that combines biochemical and mechanical factors to describe in detail the endochondral ossification process in embryonic stages. This study provides information about the influence of dynamic loading and molecular concentration in the endochondral ossification of long bones during embryonic stages, and the results are in accordance with other computational models which conclude that PTHrP, Ihh and VEGF are sufficient to capture effects of temporal variations in growth factor expressions on bone development [28], [117], [137]. Accordingly, this mechanobiological model may predict efficiently the morphogenesis of any bone rudiment based on initial cartilage configuration, applied load, frequency, signal and main molecules that govern the ossification process. However, experimental data such as molecular concentrations, molecule rate diffusions, mechanical loading magnitudes from both mice genres and other animal species are required to validate the computational model and make a much more predictive scenario. This work has a novel implementation, which uses a multiscale model to simulate in different scales of time the reaction-diffusion equations and the poroelastic formulation. This multiscale model is useful since it allows the observation of how the fluid velocity affects the growth and shape of a bone during the embryonic development when a mechanical load is being applied. Additionally, this novel formulation may be dynamic because it allows to change or to inhibit molecules and loads in order to simulate abnormalities in early stages of bone development.

\subsection{Concluding Remarks}

Bone morphogenesis obtained from the computational model developed were comparable to the morphological characteristics observed in normal bone formation during mice embryological development. In fact, the model roughly predicts the morphology of a femur highlighting its main features such as the projection of a femoral head, the apparition of the greater trochanter that form the external part of the bone and the presence of two oblong prominences, the condyles. Apart from predicting the bone morphology, the model also recreates the formation of the internal structures of the bone such as the POC, bonce collar and growth fronts. The correct formation of these 
structures was reached because of the interaction of the negativefeedback loop between PTHrP and Ihh and the diffusion of VEGF within the anlage. 



\section{CHAPTER 4}

\section{Biophysical stimuli: a review of electrical and mechanical stimulation in hyaline cartilage}





\subsection{Summary ${ }^{3}$}

Hyaline cartilage degenerative pathologies induce morphologic and biomechanical changes resulting in cartilage tissue damage. In pursuit of therapeutic options, electrical and mechanical stimulation have been proposed for improving tissue engineering approaches for cartilage repair. The purpose of this review was to highlight the effect of electrical stimulation and mechanical stimuli in chondrocyte behavior. Design. Different information sources and the MEDLINE database were systematically revised to summarize the different contributions for the past 40 years. Results. It has been shown that electrical stimulation may increase cell proliferation and stimulate the synthesis of molecules associated with the extracellular matrix of the articular cartilage, such as collagen type II, aggrecan and glycosaminoglycans, while mechanical loads trigger anabolic and catabolic responses in chondrocytes. Conclusion. The biophysical stimuli can increase cell proliferation and stimulate molecules associated with hyaline cartilage extracellular matrix maintenance.

\subsection{Introduction}

Hyaline cartilage is an avascular tissue characterized by having abundant extracellular matrix (ECM) that is mainly composed of collagen type II and proteoglycans (PGs) [5], [9], [44]. This tissue is formed by a unique cellular type, the chondrocyte, which is responsible for maintaining the integrity of the ECM [3], [4]. Within the skeletal system, hyaline cartilage is found in two specialized structures of the long bones: the articular cartilage and the growth plate [4], [5]. The first acts as a smooth, lubricated, low-friction surface that receives mechanical loads and facilitates motion between opposing joints [1], [138]; while the second, which is located between the epiphysis and

\footnotetext{
${ }^{3}$ The review presented in this chapter has been published in: J. J. Vaca-González, J. M. Guevara, M. A. Moncayo, H. Castro-Abril, Y. Hata, and D. A. Garzón-Alvarado, "Biophysical Stimuli: A Review of Electrical and Mechanical Stimulation in Hyaline Cartilage”. Cartilage, Sep. 2017.
} 
diaphysis, is responsible for the longitudinal growth and shape of long bones [7], [8].

Histologically, both articular and growth plate cartilages are tissues stratified in different zones according to chondrocytes phenotype and spatial arrangement of the ECM. Accordingly, articular cartilage is organized in 5 zones: superficial, intermediate, medium, deep, and calcified [10], whereas the growth plate is arranged into 3 zones: reserve, proliferative, and hypertrophic [15]. In both tissues, the molecular structure of the ECM within the hyaline cartilage provides dimensionality, elasticity and strength to the tissue [9], [10].

Given its location, hyaline cartilage is exposed to mechanical loads that generate different signals implicated in the physiological regulation of chondrocytes behavior and several pathological processes, considering that chondrocytes sense and respond to different mechanical loads [44], [45], [47], [139]. For example, mechanical loading has been shown to affect cell deformation, fluid flow, nutrient and ion concentration gradients, $\mathrm{pH}$ changes, and anabolic and catabolic activity of ECM components and stimulation of growth factor synthesis [140]-[145]. In turn, overweight or strong impact loading have been proven to cause damage in the ECM and chondrocyte apoptosis, resulting in dysfunction of the tissue and subsequent damage [1], [45], [52], [146]. Several studies have shown that hyaline cartilage behavior is also affected by electrical and electromagnetic stimuli, resulting in changes in cell dynamics, such as migration [147], differentiation [148]-[150], morphology [151]-[154], proliferation [155]-[160], and gene expression [161]-[174]. This implies that mechanical, electrical, and electromagnetic stimulation may have an important role in the regulation of hyaline cartilage in both normal and pathological conditions.

Based on the above, understanding the influence of biophysical stimuli in the biology of hyaline cartilage not only provides useful information regarding their role in cartilage physiology in vivo but also supplies new tools for tissue engineering and regenerative medicine focused on the development of new therapeutic approaches for the treatment of injuries 
in articular cartilage and growth plate. In this context, we performed a review of literature of the past 40 years of in vivo and in vitro studies, highlighting their results, advances, main limitations, and perspectives. The information presented summarizes the current understanding about the role of both the electrical stimulation over chondrocytes intended for articular cartilage recovery and the mechanical stimuli over growth plate and its influence on biological events during human bone development.

\subsection{Electrical stimulation for tissue engineering hyaline cartilage}

\subsubsection{Electric fields}

Electrical stimulation has been used to improve chondrocyte proliferation rate and the synthesis of characteristic ECM molecules. Electric fields (EFs) for cartilage regeneration have been used in in vivo and in vitro systems (Table 4-1) [155], [159], [162], [174]-[177].

\subsubsection{In vivo studies}

The first reported use of electrical stimulation to influence cartilage was in 1974 by Baker et al., who assessed the influence of a bimetallic platinum electrochemical device in the recovery of full thickness injuries of articular cartilage. The electrochemical device was implanted in the lateral femoral condyle at the location of the injury and voltages from 15 to $500 \mathrm{mV}$ were applied. Results showed a $71 \%$ of increase in the production of ECM in treated animals [176]. This finding set the basis for the application of electrical therapy in patients presenting joint pathologies. The experience reported up to now in the literature has evidenced that the application of the EFs lead to pain relief in patients with knee osteoarthritis. For instance, Farr et al., used a transcutaneous electric stimulator in 288 patients with knee osteoarthritis. Patients who received electrical stimulation for more than $750 \mathrm{~h}$ (73\% of total population) reported both pain relief and a 
decrease in anti-inflammatory drug use (45.3\%). Moreover, the therapy delays the need of a surgical intervention in chronic patients [177].

\subsubsection{In vitro studies}

The technique of delivering electrical stimulation to cell culture plates consists of an indirect coupling system that uses external parallel electrodes connected to a power supply (Figure 4-1A) [155], [162], [167], [178]. The custom designed devices are noninvasive capacitive coupling systems that can be used to stimulate any cellular type, and they are based on the same principle used in medical stimulation devices [175], [177], [178].

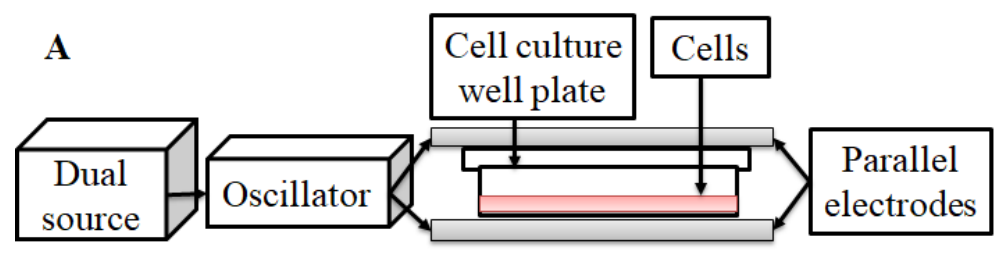

B

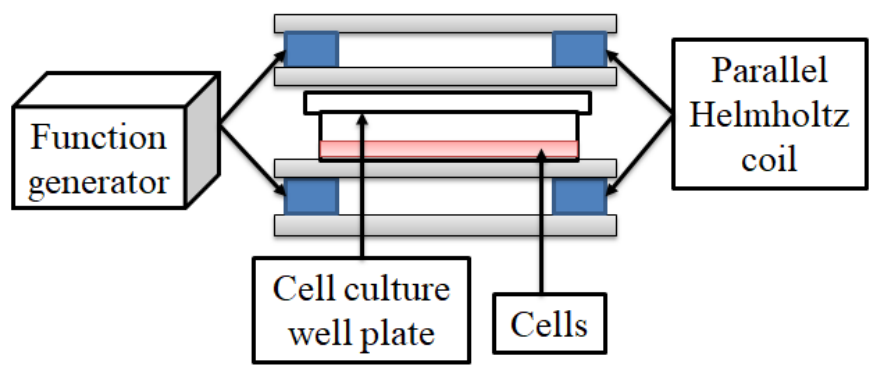

Figure 4-1: Schematic diagram of an indirect coupling system to stimulate in vitro cell cultures.

A) Configuration to apply EFs to the culture medium between the parallel electrodes. B) Configuration to apply electromagnetic fields to the culture medium between the parallel electrodes.

In vitro, the application of EFs to monolayer cultures has shown to increase cell population and to stimulate synthesis of main molecules that compound cartilage such as collagen type II, GAGs, and aggrecan. 


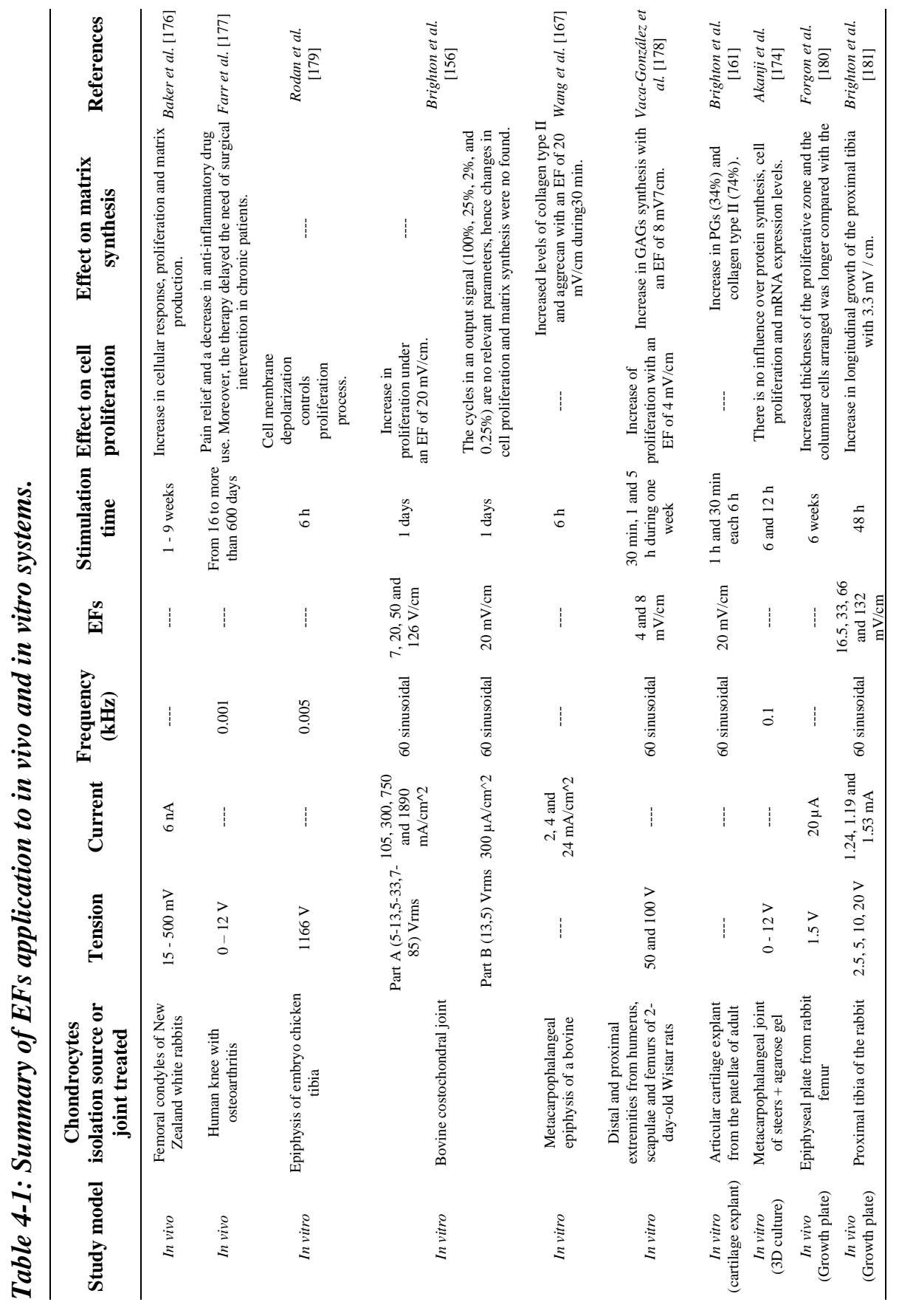


For instance, Rodan et al., demonstrated that increased chondrocyte proliferation observed after EFs stimulation was triggered by a depolarization in the cell membrane, it means that the $\mathrm{Na}+$ and $\mathrm{Ca} 2+$ fluxes generated by the EFs trigger DNA synthesis of chondrocytes [179]. More descriptive studies were carried out by Brighton et al., in which they stimulated chondrocytes with different voltage values (10, 100,250 , and $1000 \mathrm{~V}$ ) to a frequency of $60 \mathrm{kHz}$ (sinusoidal-wave form). Their results indicated that voltages of 10 and $1000 \mathrm{~V}$ decreased cell proliferation; while a voltage of $250 \mathrm{~V}$ increased the GAGs synthesis [158]. In these studies, no data about the magnitude of the EFs were reported.

Similar studies were performed by Armstrong and Brighton where chondrocytes were stimulated with several EFs (15, 22.5, 30, and 45 $\mathrm{mV} / \mathrm{cm}$ ). Their results demonstrated an increase in cell population when EFs of 15, 22.5, and $30 \mathrm{mV} / \mathrm{cm}$ were applied. An increase in GAGs synthesis (66\%) was obtained after stimulation with EFs of $45 \mathrm{mV} / \mathrm{cm}$ [155], [157]. From these studies, it was possible to conclude that an EF close to $20 \mathrm{mV} / \mathrm{cm}$ stimulates chondrocyte dynamics. Brighton et al., in later studies reported that an EF of $20 \mathrm{mV} / \mathrm{cm}$ increases the cell proliferation by $50 \%$ [156]; while Wang et al., in a more detailed study elucidated that this EF of $20 \mathrm{mV} / \mathrm{cm}$ increases aggrecan and collagen type II synthesis [167]. On the contrary, researchers such as Nakasuji et al., have applied EFs of greater magnitude (50, 100, 250, and 500 $\mathrm{mV} / \mathrm{cm}$ ) to chondrocytes cultured in vitro. The results showed that EFs of greater magnitude also affect cell proliferation and ECM synthesis [159]. Because of the discrepancy regarding the magnitude of the EFs required to stimulate the in vitro cultures, Vaca-González et al., implemented a new methodology to calculate EFs and modulate proliferation and GAGs synthesis. Results allowed to establish the dielectric constant of the cell culture medium (complex permittivity and conductivity), and also showed that EFs of $4 \mathrm{mV} / \mathrm{cm}$ applied during 30 min increment cell population; while EFs of $8 \mathrm{mV} / \mathrm{cm}$ applied during 5 $\mathrm{h}$ maintain a stable GAGs synthesis [178]. 


\subsubsection{Studies performed in explants and 3-Dimensional cartilage constructs}

The EFs have also been applied to cartilage explants and tridimensional constructs [60]. Brighton et al., stimulated cartilage explants with EFs of $20 \mathrm{mV} / \mathrm{cm}$. Results showed an increase in PGs (34\%) and collagen type II (71\%) after 14 days of stimulation [161]. Because EFs of 20 $\mathrm{mV} / \mathrm{cm}$ have been shown to influence the cellular dynamics, the research group of Brighton et al., studied the effect of the electrostimulation in osteoarthritic human cartilage explants. In this study, osteoarthritic explants stimulated for 14 days using $20 \mathrm{mV} / \mathrm{cm}$ EFs showed 1.4 and 1.5-fold increase in PGs and collagen type II, respectively [162]. EFs have been also applied to 3-dimensional (3D) cartilage constructs used for cartilage repair; however, to our knowledge, the reports in literature are limited and the results have been contradictory. For instance, Szasz et al., stimulated 3D chondrocyteseeded agarose gels, obtaining increases in cell density and GAGs synthesis [173]. In contrast, Akanji et al., investigated the effects of direct current on cell proliferation and matrix synthesis, using a 3D chondrocyte-agarose model system. Their results demonstrated that EFs have no influence over protein synthesis, cell proliferation, and mRNA expression levels [174].

\subsubsection{Effect of EFs over the growth plate}

Applying electrical stimulation across growth plate cartilage has little effect on composition [180], but can alter the thickness of zones within the cartilage [180], [182], and ultimately the length of the bone [180], [183]. However, high voltage reduces bone growth and maintains cartilage in a quiescent state [183].

\subsubsection{Electromagnetic fields}

Similar to EFs, electromagnetic fields (EMFs) have been applied in in vivo and in vitro studies using cartilage and growth plate explants, and tridimensional structures (Table 4-2) [151], [152], [154], [165], [170], [172], [184]. 


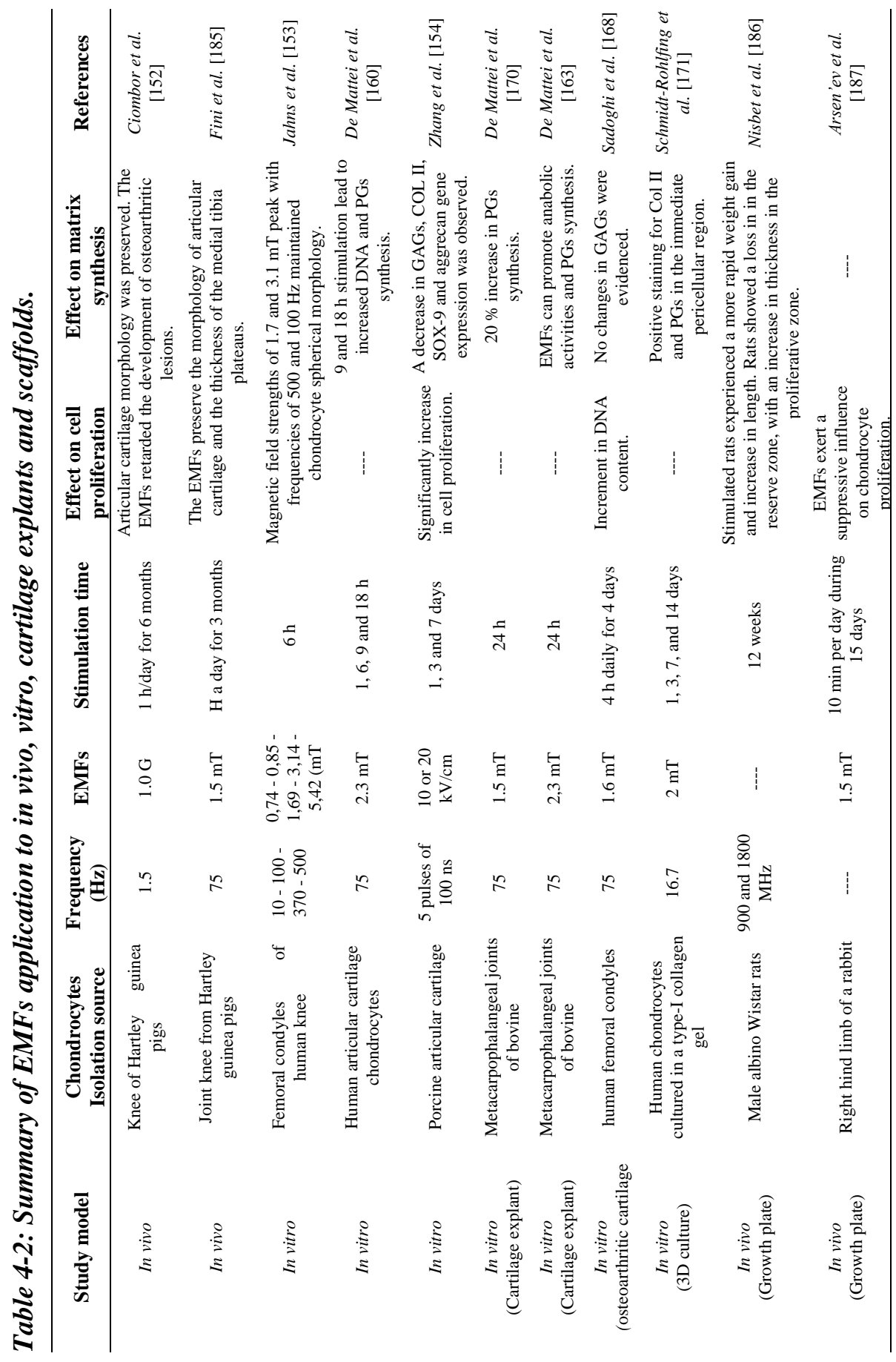


Similar to EFs, the technique of deliver EMFs consists of an indirect coupling system that uses external parallel coils connected to a function generator (Figure 4-1B) [152], [153], [163], [184]. This kind of noninvasive configuration has the advantage of generating small currents and potentials in the proximity of the targeted cells, and also it generates a homogeneous EMF across the cell culture [175].

\subsubsection{In vivo use of EMFs to heal osteoarthritis}

In vivo animal models have been used to study the influence of EMFs in the recovery of knee osteoarthritis. Ciombor et al., showed that articular cartilage morphology was preserved, and the development of osteoarthritic lesions were retarded when EMFs were applied [152]. EMFs have also been implemented in clinical studies to treat knee osteoarthritis. Results have evidenced that electromagnetic stimulation contributes to preserve articular cartilage morphology [184], [185], improvement in joint motion, tenderness, and reduction in pain [188][191].

\subsubsection{In vitro studies using cell cultures}

The application of EMFs in monolayer cultures have shown preservation of chondrocytes morphology [153], increased DNA synthesis [160], and enhancement of proliferation and GAGs synthesis in human chondrocytes [192]. It has also been demonstrated that EMFs significantly increase cell population but decrease the synthesis of characteristic molecules of hyaline cartilage such as collagen type II, aggrecan, SOX-9, and GAGs [154].

\subsubsection{Studies performed in explants 3-Dimensional cartilage constructs}

Healthy and pathological tissue explants have also been treated with EMFs [165], [168], [170]. The use of EMFs over cartilage explants have shown increase in PGs synthesis and stimulation of anabolic activities [163], [170], while the effect of EMFs in osteoarthritic explants have evidenced an improvement in osteoarthritis of grade I and 
III, increasing PGs synthesis and counteracting catabolic activities [165]. However, it was also shown that EMFs have no effect on DNA and PGs synthesis [168]. The application of EMFs has also been investigated in osteoarthritic chondrocytes cultured in 3D structures. Results showed that the PGs concentration of osteoarthritic chondrocytes cultured in alginate scaffolds was stored in the cell culture medium after stimulation was finished [151]. Normal articular chondrocytes cultured in 3D constructs were also stimulated with EMFs. In studies performed by Schmidt-Rohlfing et al., and Nicolin et $a l$, the chondrocytes that were cultured in a collagen membrane showed that this scaffold is a useful bioengineering construct since allowed increases in cell proliferation, collagen type II synthesis, and PGs in the immediate pericellular region [171], [172].

\subsubsection{Effect of the EMFs over the growth plate}

Studies have been carried out to study the effects of EMFs over growth plate development. A recent study showed that male albino rats stimulated with EMFs experienced a more rapid weight gain and increase in length. Moreover, the stimulated rats showed a loss in cartilage matrix density in the reserve zone, with an increase in thickness of the reserve and proliferative zones. In addition, measurements of the growth plate thickness showed that the trabecular zone was thinnest, and the reserve and proliferative zones were thickest. There were no significant differences between the groups with respect to the thickness of the hypertrophic zone [186]. A similar study evidenced that EMFs exert a suppressive influence on chondrocyte proliferation in the growth plates and promote expansion of the differentiate fraction [187]. The epiphyseal plate has been also stimulated through in vitro cultures; for instance, the costochondral junction growth plate of a rat was exposed to different EMFs and changes of temperature. Results indicated that longitudinal growth of the costochondral junction was only observed when EMFs were applied in an environment where the temperature increased [193]. 


\subsection{Mechanical Stimulation for Tissue Engineering Hyaline Cartilage}

\subsubsection{Compressive loads}

Because of the fact that hyaline cartilage, especially the one found in joints and growth plate, is mainly subjected to compressive mechanical stimuli [146], [194], [195], several in vivo and in vitro studies have been performed in order to identify the effects of mechanical compression in cell dynamics (proliferation, hypertrophy, and apoptosis) [5], [106], [196]-[202], cell height [106], [197], [203], and the biosynthetic behavior of chondrocytes, assessed mainly in terms of expression of molecules of the ECM (collagen types II and X and aggrecan) and some enzymes (MMP 13 and ADAMTS-4/5) [204], [205]. In addition, changes in the expression of metalloproteinase inhibitors (TIMP-1 and TIMP-2), growth factors (VEGF), and other molecules are influenced by load (Table 4-3) [141], [202], [206]-[208]. Up to now the specific mechanisms involved in mechanotransduction are not well understood; however, two kind of proteins have been involved in these processes: integral membrane proteins associated in ECM interactions (e.g., integrins) and mechanosensitive ion channels [138], [209]-[211]. Furthermore, signaling mediated by the primary cilium and MAPK pathway have also been implicated [212]-[217]. Regarding the devices used to apply compressive loads, the technique of deliver mechanical loads consist of a system that uses loading actuators that exert an axial force (Figure 4-2A) [138], [214], [216]. These devices present the advantage that maintain the cell cultures in atmospheric conditions of $37^{\circ} \mathrm{C}$ and $5 \% \mathrm{CO}_{2}$ while mechanical loads are applied [218].

\subsubsection{In vivo studies}

To understand the effect of mechanical loading on chondrocytes behavior within the tissue, some in vivo studies have been performed in both articular cartilage and growth plate. 


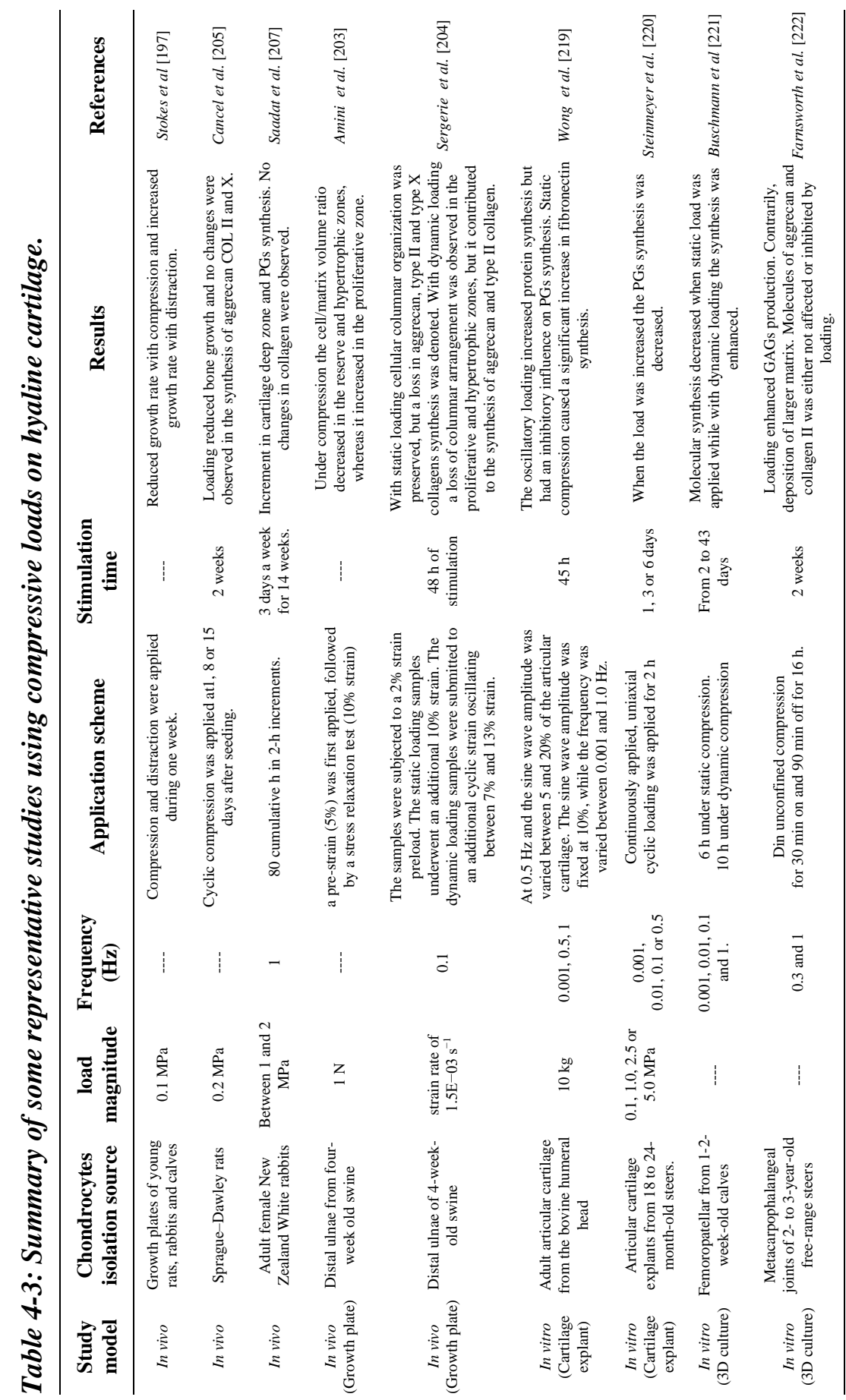


A

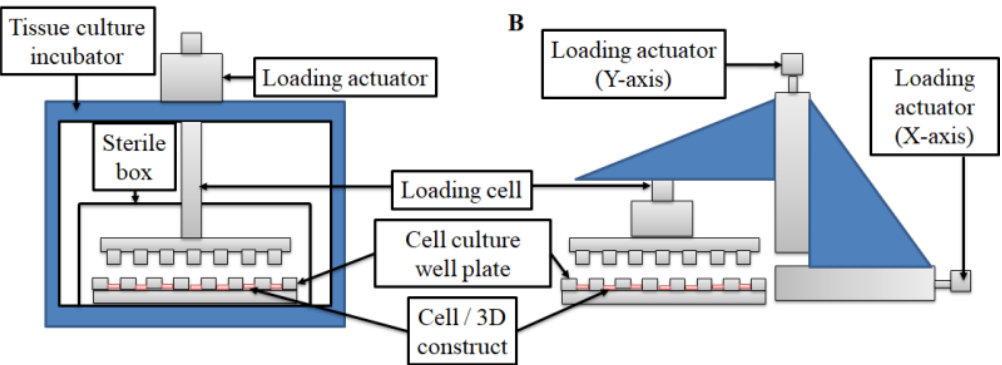

Figure 4-2: Schematic diagram of the devices used to apply mechanical loads to chondrocytes cultured in 3 dimensional (3D) structures. Both schemes have in common a loading cell, a cell culture well plate and the $3 D$ construct.

A) Configuration to apply compressive loads to $3 D$ cell cultures. This device uses a tissue culture incubator and a sterile box to maintain cell cultures in humidified atmosphere. B) Configuration to apply compressive and shear stress to the chondrocyte culture. This device uses two loading actuators to apply loads in the $X$ and $Y$ axis.

The studies performed in articular cartilage have mainly focused in analyzing the synthesis of ECM molecules as PGs, where a direct correlation between loading in the joint and PGs levels within the tissue was observed [138], [141], [207], [217]. Several studies performed in the growth plate have assessed the effect of mechanical loading as a regulator of bone growth and ossification. Moreover, alterations of mechanical modulation have been associated to several clinical conditions, including the angular progression deformities in tibia (Blount disease), and the development of scoliosis [106], [196]. Studies using chicks and rodents have demonstrated that mechanical stimulation of bone rudiments during embryological development plays an important role in normal growth and bone morphogenesis [223].

At cellular level, studies using rodents have shown that mechanical loading induces histological changes within the growth plate, specifically in the width of the proliferative and hypertrophic zones [197]-[201]. Additionally, it has been observed a decrease in collagen type II and aggrecan concentrations in growth plates subjected to long term static compressions [205]. 


\subsubsection{In vitro studies: tissue cultures}

As an alternative model to study the influence of mechanical loading over chondrocytes behavior, in vitro protocols applying compressive loads to articular cartilage and growth plate explants have been performed. For articular cartilage, the reported results of ECM molecules synthesis under dynamic loading are contradictory with some studies showing an increase while others indicate either a decrease or no changes after loading [106], [195], [202], [207], [219], [220], [224], [225]. For growth plate, studies using tissue explants have shown differential morphological changes of chondrocytes in the different zones under compression [203]. Additionally, under shortterm compressive loads, a decrease in aggrecan, collagen type II and X has been reported. In contrast, under dynamic loading no changes in ECM composition although changes in cell organization were observed [204].

\subsubsection{In vitro studies: cell cultures}

Given the difficulties for analyzing the individual effects of mechanical stimuli to which cartilage is subjected in vivo, the response of chondrocytes has been evaluated in monolayer and 3D cultures [141], [146], [194], [195], [215], [219], [220]. These analyses not only provide information about the cellular responses and the regulatory mechanisms associated, but also they allow to evaluate the use of mechanical stimuli in tissue engineering approaches [141]. The effect of compressive loads in in vitro cultures of chondrocytes depends up on load's intensity and duration [138]. In this context, it has been observed that the application of static loads increased the production of several types of MMPs, and also inhibited the synthesis of collagen type II and PGs [138], [217]. In turn, the effects of dynamic loading in cell proliferation and synthesis of PGs, GAGs, and collagen type II are contradictory [138], [194], [222], [224]-[232], [195], [233]-[238], [202], [207], [217]-[221]. This behavior may be related to the wide variability of the methodology used for each group, which can be related to different sources of chondrocytes, age of the animal model, characteristics of 3D matrix used, chondrocyte seeding density, frequency of the stimulus, load 
cycles, and initiation and duration of the stimulation [138], [207], [235], [218], [219], [221], [222], [226]-[229].

\subsubsection{Tension}

The chondrocytes response to tensile loading have also been addressed using in vitro monolayer cultures. Such cultures have been performed by seeding cells in membranes that are submitted to uniaxial or biaxial dynamic stretching. Most of the studies have evaluated chondrocyte dynamics in terms of cell proliferation and molecular synthesis of collagens and PGs. Similar to that observed for compressive loading, the evidence available suggests that the response of chondrocytes to tensile loading also depends on the duration and intensity of the stimulation [101], [142], [239], [240]. In fact, anabolic effects in chondrocytes are induced after short-term simulation (less than $12 \mathrm{~h}$ ); while a decrease in the ECM molecules is only observed after a prolonged stimulation [142]. In addition, some studies have reported an increase of other molecules such as proteases and their inhibitors (e.g., TIMPs, MMPs), soluble factors (e.g., TGF $\beta$, VEGF, PTHrP), and pro inflammatory factors (e.g., nitric oxide, prostaglandin E, cyclooxygenase 2) after tensile loading stimulation [100]-[102], [142], [215], [240].

However, it is difficult to extrapolate definitive conclusions regarding the stimulus characteristics and chondrocytes response considering that, similarly to compressive loading, there is a high variability in the stimulation protocol used among different studies in terms of strain magnitude, loading duration and frequency (reviewed in Bleuel et al., [142]).

\subsubsection{Hydrostatic pressure}

\subsubsection{In vitro studies: tissue cultures}

Hydrostatic pressure (HP) has been extensively used as a stimulus to induce changes over hyaline cartilage such as protein expression and matrix production [241]. However, the mechanism by which HP 
produce those changes is not well understood. HP provides a robust method of chondrocyte stimulation since it can be applied over cells cultured in monolayer, 3D engineered constructs or cartilage explants (Table 4-4) [242], [243]. Several studies have shown the relation between HP and biological response of hyaline cartilage, such as investigations performed by Parkkinnen et al., have shown a microtubule-dependent compaction of the Golgi apparatus and decrease in GAGs synthesis [244]. In a similar study, Lammi et al., found that static HP resulted in a 37\% decrease in GAGs synthesis, reduction of aggrecan mRNA levels and synthesis of atypically large aggrecan molecules. The alteration in aggrecan size demonstrates that HP may affect the production of ECM molecules at post-translation levels [245].

\subsubsection{Studies performed in cartilage explants and 3- Dimensional constructs}

HP also has been applied over 3D scaffolds and explants. For instance, Carver and Heath [246], [247], showed that chondrocytes cultured in poly glycolic acid (PGA) meshes and stimulated with $6.87 \mathrm{MPa}$ experienced an increase in GAGs and collagen production in adult cells, whereas the same magnitude of HP had an increase of only collagen synthesis in juvenile cells. There are no differences of applying HP either in cartilage explants or in chondrocytes cultured in vitro; however, the magnitude of the pressure and the frequency are relevant factors to stimulate PGs synthesis [248].

\subsubsection{Shear stress}

Within the joint and growth plate chondrocytes are exposed either to compressive HP or deviatory stresses such as shear stress.135 Previous studies have indicated that chondrocytes are exposed to different levels of fluid flow within the tissue [249], [250], suggesting that mechanical shear stress has a pathophysiologic relevance in cartilage biology [251]. Within this context, several studies have analyzed the influence of such stimuli on chondrocyte behavior. 


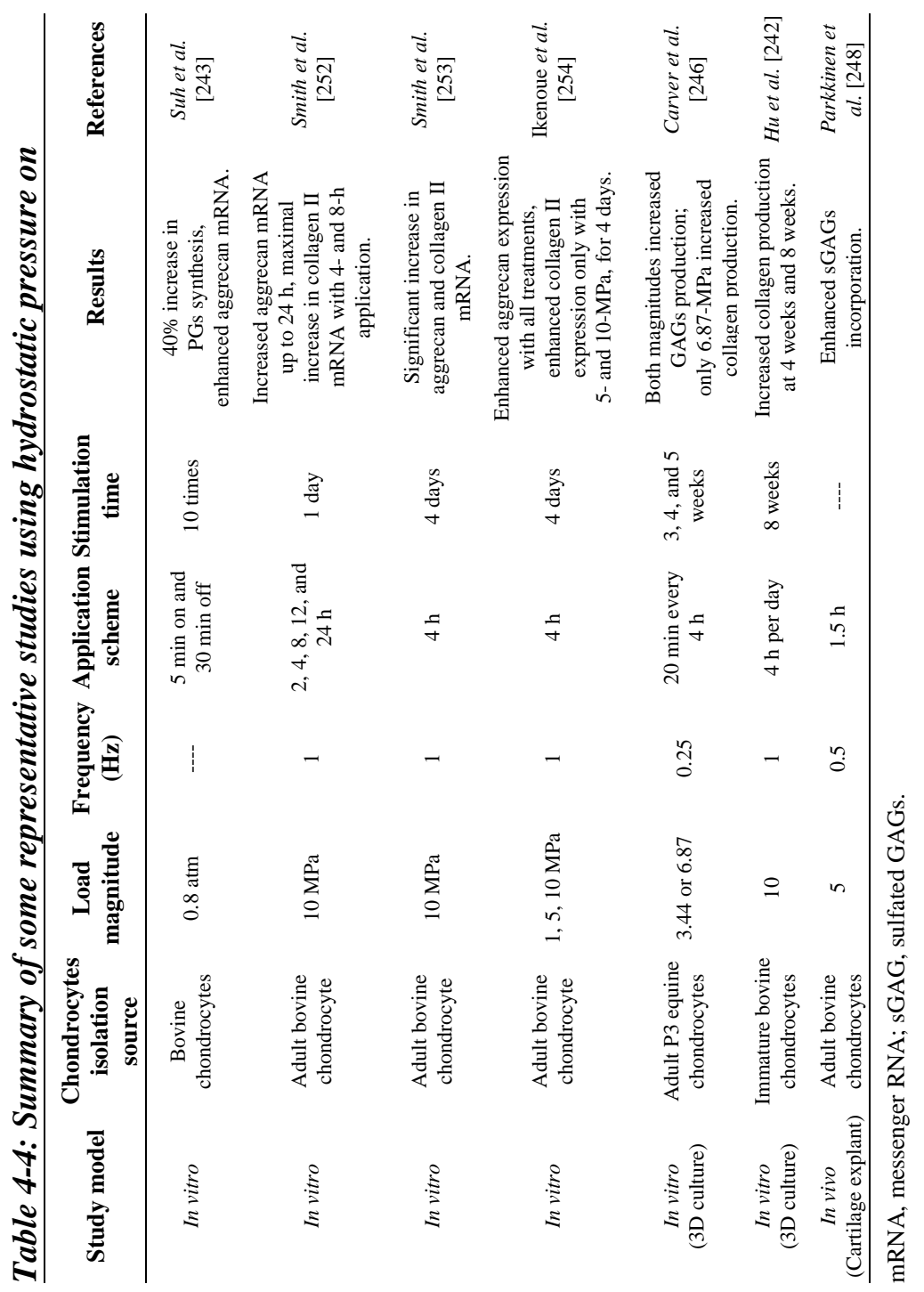

For instance, Mohtai et al., applied shear stress over human articular chondrocytes seeded in a high monolayer culture. Cell cultures were stimulated with loads of $0.16,0.41,0.82$, and $1.64 \mathrm{~Pa}$ at rotating velocities of 20, 50, 100, and $200 \mathrm{rpm}$. Results indicated that this stimulation scheme increased the release of pro-inflammatory mediators and nitric oxide, decreased the expression of aggrecan and collagen type II, and induced molecular changes associated with 
apoptosis [255]. Additionally, it is known that hyaline cartilage tissue engineering constructs, are also affected by shear stress, revealing that this stimulus may alter the intercellular signaling pathways in chondrocytes [246], [254]. Some reports suggest that fluid shear stress reduces expression of aggrecan and collagen type II [256].

Other studies, like those developed by Waldman et al., used a stimulation scheme based on $2 \%$ of shear stress amplitude at $1 \mathrm{~Hz}$ for 400 cycles every second day, and demonstrate that an intermittent application of dynamic shearing forces during 4-week periods improved the quality of the cartilaginous tissue formed in vitro. These data indicate that the nature and magnitude of shear stress may play a significant role in the homeostasis of the structure and function of hyaline cartilage [257]. For this reason, the cellular mechanisms underlying the responses of articular chondrocytes to mechanical stresses are important for understanding the pathogenesis of several hyaline cartilage diseases [251]. Similar to compressive loads, the technique of deliver the shear stress consists of a loading actuator that generate forces along the $X$ and $Y$ axis at the same time (Figure 4-2B) [255], [257]. In this context, this configuration allows one to apply precise strains on multiple axis similar to the mechanical environment supported by the joint [258].

\subsection{Cellular mechanisms involved in transduction of biophysical stimuli}

In chondrocytes, biophysical stimuli are sensed mainly by membrane proteins involved in ECM-cell interactions and ionic channels [138], [259], [260]. In addition, some stimuli may induce changes in the cytoskeleton [147], [261].

ECM-cell interactions are associated mainly with the transduction of mechanical stimuli, since they allow cells to sense conformational changes of the ECM that trigger, intracellularly, the activation of different cell signaling cascades leading to specific cell responses [138], [259]. The main proteins associated with ECM-cell interaction are 
integrins, integral membrane proteins that bind to several ECM molecules with different affinities [259], [262], [263]. The main integrins expressed by chondrocytes are $\alpha 1 \beta 1, \alpha 3 \beta 1, \alpha 5 \beta 1, \alpha 10 \beta 1$, $\alpha v \beta 3$, and $\alpha v \beta 5$, which act as receptors for collagen types VI and II, matrilin-1, fibronectin, osteopontin, COMP, and vitronectin, respectively [210], [211], [259], [262], [264]. Cytoplasmic domains of integrins are coupled to kinases that have been implicated in signal transduction through Ras, Rho, and Rac pathways [215], [217]. In addition to integrins, other membrane proteins like CD44 and annexin $\mathrm{V}$ (also known as anchorin CII) have also been associated in ECM-cell interactions in chondrocytes. CD44 is a transmembrane hyaluronanbinding glycoprotein that is involved not only in anchoring but also in sensing and signaling functions [264]-[266]. Annexin V is a member of a family of calcium and phospholipid binding proteins that binds mainly with collagens present in the PCM and ECM, mainly type II and with lesser affinity to types V, IX, X, and XI [265]-[267].

On the other hand, ion channels have been associated to both mechanical and electrical signal transduction. Although a plethora of ion channels have been identified in chondrocytes membranes, calcium channels are the main type of ion channels associated to transduction of biophysical stimulation [138], [209], [217]. The calcium channels most associated to these processes include voltage-gated sodium channels, voltage-gated calcium channels, and stretch activated ion channels. In addition, various members of the TRPV (transient receptor potential vanilloid) nonselective cationic channels family, especially TRPV4, have been associated to osmotic stress responses [138], [209], [217]. In fact, it has been described that in chondrocytes, cell deformations caused either by changes in cell volume or due to mechanical loading, lead to activation of different kinds of ionic channels, and some studies have evidenced changes in intracellular calcium levels in chondrocytes after mechanical loading [138], [213], [214], [216], [217]. Electrical signals applied either in direct or indirect contact with the cells, exert their effect on the cell membrane by activating the voltage-gated calcium channels leading to increase in the intracellular $\mathrm{Ca}^{2+}$ levels [167], [176], [268]. 
Finally, as previously mentioned, some stimuli are associated to conformational changes of the cytoskeleton. That is the case of electrical stimulation, which has proven to induce changes in actin filaments causing cytoplasm elongation followed by a perpendicular alignment with regard to the applied EFs [261]. Furthermore, electrical stimulation has shown to promote cell movement, process known as galvanotaxis [147].

\subsection{Perspectives}

This review focuses on the effect of biophysical stimuli in chondrocyte behavior, showing that electrical and mechanical stimulation have important roles in physiology of hyaline cartilage. In addition, biophysical stimuli display interesting potential as additional tools for both tissue engineering and regenerative medicine, toward the development of new therapeutic alternatives for cartilage pathologies. The effects of biophysical stimulation on chondrocytes dynamics have been explored in vivo and using several in vitro approximations. In monolayer cell cultures, stimulation with EFs, EMFs, HP, and tension can promote chondrocyte proliferation and molecular synthesis [142], [175], [184], [252], [254]. Considering this, with the current use of scaffolds combined with the application of biophysical stimuli it is possible to obtain tissue constructs that mimic in vivo characteristics of cartilage.

For both electrical and mechanical stimuli, different frequencies, intensities, and duration of stimulation schemes have been tested; however, the magnitudes of the stimuli that directly stimulate chondrocytes in those cultures have been poorly explored. Regarding electrical stimulation, at present, calculations of cell culture media dielectric constants have been recently reported. These electric constants, such as permittivity and conductivity, are essential parameters to calculate EFs and EMFs [178], [269]. Thus, this leads to a new research area that will allow to implement a better methodology to calculate the EFs and EMFs in a more detailed way. In electrical stimulations, there are many discrepancies in the obtained results because one of the limitations is the required high voltage to generate 
the EFs, the stimulation time to achieve the best rate of proliferation and molecular synthesis, as well as the amount of days that cell cultures need to be under stimulation. For this reason, electrical stimulation for tissue engineering hyaline cartilage still is an open research area. On the other hand, little is known of the mechanical environment and the effects on fluid flow and nutrient availability within cartilage or in vitro 3D cultures of the different types of mechanical stimulation. Recently, there have been some approaches that use computational modeling to approach such issues [270]-[274]. This is a promising field that may improve the understanding of the effects of mechanical stimuli on chondrocyte behavior as well as provide powerful tools for scaffold designs for 3D in vitro culture systems and mechanical stimulation scheme selection. Furthermore, biophysical stimuli could help to enhance cell proliferation, differentiation, and ECM deposition, opening up new possibilities in 3D articular cartilage and growth plate tissues, and allowing to design novel tissue-engineering constructs similar to real life.

Given the good results obtained using some protocols of electrical and mechanical stimulation independently for in vitro hyaline cartilage tissue engineering, the integration of these both methods is currently been studied [166]. This leads to a new field of research that will focus on stimulation of cartilage explants or 3D constructs with 2 biophysical stimuli simultaneously. As a novel and potential tool, it would be possible to create special devices that generate distinct electrical and mechanical intensities according to the necessity of a particular treatment. This contributes to tissue engineering because it is noteworthy that biophysical stimuli are the basis for the next generation of cartilage regeneration technology, since those electrical and mechanical stimuli help the development of biomimetic samples that recreate an environment where the functional, structural, and biological features of hyaline cartilage remain stable at the time of cartilage replacement. 



\section{CHAPTER 5}

An in vitro chondrocyte
electrical stimulation framework 



\subsection{Summary ${ }^{4}$}

The electric fields have been used as external biophysical stimuli to increase cell population and stimulate the molecular synthesis of chondrocytes. However, in the methodology reported by different researchers there is no evidence about the procedure to calculate electric fields. This work presents a new framework to calculate electric fields and modulate proliferation, cell death and glycosaminoglycan synthesis. For this study, we used a computational analysis, by a finite element method for experimental setting, to find the dielectric constants of the cell culture medium and the experimental geometrical configuration. Chondrocytes were stimulated with electric fields of 4 and $8 \mathrm{mV} / \mathrm{cm}$, and for each of these electric fields, three exposure times were tested ( $30 \mathrm{~min}, 1 \mathrm{~h}$ and $5 \mathrm{~h}$ ). We observed an increment in cell population when an electric field of $4 \mathrm{mV} / \mathrm{cm}$ was applied during 30 min. The GAGs synthesis remained stable after an electric field of 8 $\mathrm{mV} / \mathrm{cm}$ was applied during $5 \mathrm{~h}$. No changes in cell population and molecular synthesis were perceived when electric fields were applied during $1 \mathrm{~h}$. This approach is a promising tool that could be used to enhance in vitro procedures in order to obtain well characterized chondrocyte cell cultures either by increasing cell proliferation or stimulating molecular synthesis.

\subsection{Introduction}

Articular cartilage is a connective tissue located at the ends of bones in the articular surfaces. This tissue receives multiaxial loading and the majority of load bearing and mechanical compliance is accomplished by the trabecular bone. Articular cartilage acts as a smooth, lubricated, low-friction surface that facilitates motion between opposing joint surfaces [1], [138]; therefore, given its damping function, articular

\footnotetext{
${ }^{4}$ The results presented in this chapter has been published in: J.J. Vaca-González, J. Guevara, J. Vega, and D. Garzón-Alvarado, "An In Vitro Chondrocyte Electrical Stimulation Framework: A Methodology to Calculate Electric Fields and Modulate Proliferation, Cell Death and Glycosaminoglycan Synthesis”. Cell. Mol. Bioeng., pp. 1-11, 2016.
} 
cartilage requires a specialized molecular structure to resist mechanical loads. Thus, the extracellular matrix (ECM) of the articular cartilage contains as main molecules collagens and PGs [1], [9], [275]. Moreover, this tissue is characterized by low cell density, where the only cellular type is the chondrocyte [1].

Articular cartilage is an avascular tissue that has limited self-healing properties when it is affected by traumatic injuries. In such cases, articular cartilage is replaced by fibrous tissue also known as fibrocartilage; nevertheless, this type of joint surface is mostly inefficient for load bearing and has a loss in friction properties [1], [46], [275]-[277]. Given that articular cartilage is subject to degradation when is affected by injuries, novel therapeutic options have been developed for its repair [1], [9], [46], [278]-[282]. For instance, the autologous chondrocyte implantation expands the patient's chondrocytes through in vitro cultures; once enough chondrocytes have been obtained, cells are re-implanted into the injured articular cartilage [46], [280], [281]. This treatment could be enhanced if an external biophysical stimulus is used to improve the chondrocyte proliferation rate and the synthesis of extracellular molecules. Studies have indicated that chondrocytes cultured in monolayer respond to stimulation with electric fields (EFs) [175]. For example, Rodan and collaborators have worked on articular cartilage chondrocytes stimulation. In their study, cells stimulated with EFs during a period of $6 \mathrm{~h}$ increased the DNA synthesis [179]. Similar researchers have been carried out in articular cartilage chondrocytes stimulated with several EFs at $60 \mathrm{kHz}$ sine wave signal. Results showed an increase in chondrocyte population and synthesis of glycosaminoglycans (GAGs), the molecules responsible for water retention inside articular cartilage [155], [157]-[159]. In addition, when using EFs of lower intensity at $60 \mathrm{kHz}$ sine wave signal, the increase in chondrocyte proliferation was evidenced, as well as the synthesis of molecules such as collagen type II and aggrecan [156], [161], [162], [167].

Even though in vitro assays have evaluated chondrocyte proliferation and articular cartilage molecular synthesis, there are many discrepancies in the obtained results. A key limitation is that the EFs 
used for direct cell stimulation, the stimulation time per day to achieve the best rate of proliferation and GAGs synthesis, as well as the amount of days that cell cultures need to be under stimulation have not been well determined; however, the frequency $(60 \mathrm{kHz})$ and wave type (sine wave-form) have been well established and were therefore considered in this research [156], [159], [161], [162], [167]. The aim of this study was to stimulate chondrocytes cell cultures with two EFs intensities (4, and $8 \mathrm{mV} / \mathrm{cm}$ ) using three different stimulation schemes (30 min, 1 , and $5 \mathrm{~h}$ ). To accomplish this, we calculated the dielectric properties of the cell culture medium, such as the complex permittivity $\left(\varepsilon_{j c m}\right)$ and conductivity $\left(\sigma_{c m}\right)$, through experimental and computational approaches. In addition, we designed a novel device based on a parallel plate capacitor, to generate and distribute the EFs homogeneously over the cell cultures. Results here obtained show that, depending on the EFs and the scheme used to stimulate the cell cultures, chondrocytes can double their initial cell population, inhibit their proliferative capacity or maintain their molecular synthesis stable. This finding can be used to improve therapies such as the autologous chondrocyte implantation, considering that the electrical stimulation of in vitro cultures may be manipulated in order to enhance the chondrocyte cell growth or the synthesis of GAGs. Furthermore, this optimization improves the articular chondrocyte culture conditions intended for future therapeutic strategies in tissue engineering.

\subsection{Materials and methods}

\subsubsection{Chondrocyte isolation and cell culture}

The distal and proximal extremities from humerus, scapulae and femurs of 2-day-old Wistar rats were isolated. The rats were obtained from the Pharmacy Faculty and sacrificed by decapitation at the Biomimetics laboratory of the Biotechnology Institute at Universidad Nacional of Colombia. Recommendations from the ethics committee were followed for this procedure. 
Taking into account the high cell density required for the experimental design, we decided to use the chondroepiphysis as it has been reported in literature [283]. This tissue is appropriate to obtain a high number of pure cells required for our study. Nevertheless, in this sample chondrocytes at different maturation stages are expected. First, the extremities were washed with PBS (Phosphate Buffered Saline) $+2 \%$ of antibiotics (streptomycin and penicillin, LONZA, Walkersville, MD USA). Next, the fibrous tissue and muscle were carefully removed from the epiphysis and metaphysis. After, the epiphysis was segmented in small pieces of approximately $4 \mathrm{~mm}$ and then washed with $\mathrm{PBS}+2 \%$ of antibiotics. The fragments were submitted to a process of double digestion using $0.25 \%$ Trypsin/EDTA (LONZA, Walkersville, MD USA) during $1 \mathrm{~h}$ at $37 \mathrm{C}$, followed by digestion using $0.3 \%$ of collagenase type I in HBSS (Hanks Buffered Saline Solution, LONZA, Walkersville, MD USA) during $4 \mathrm{~h}$ at $37{ }^{\circ} \mathrm{C}$. The reaction was inactivated with $20 \%$ of fetal calf serum (FCS). The solution was homogenized by pipetting, then filtered with a sterile nylon membrane to eliminate remaining tissue, and finally, it was centrifuged. Cells were suspended in $1 \mathrm{~mL}$ of culture medium (DMEM-F12 with $50 \mu \mathrm{g} / \mathrm{mL}$ of ascorbic acid, $100 \mu \mathrm{g} / \mathrm{mL}$ of sodium pyruvate, $1 \%$ of streptomycin/ penicillin and 1\% FCS) [156], [167]. Then, the cell yield and viability were determined by counting in hemocytometer and trypan blue dye exclusion, respectively. Despite the isolation source, chondrocytes cultured in monolayer display a progressive loss of phenotype that increase with passages with the most striking phenotypic alterations becoming evident after passage four [284]-[286]. Therefore, for stimulation assays, 45.000 cells were seeded at day zero into a six culture well plate; moreover, chondrocytes expanded in culture at passage one were used for all experiments. Cell cultures remained in atmospheric conditions of $37{ }^{\circ} \mathrm{C}$ and $5 \% \mathrm{CO}_{2}$ and the cell culture medium was changed every other day. This procedure was implemented for cultures under electrical stimulation and controls. 


\subsubsection{Chondrocyte culture medium characterization and EF estimation}

Experimental and computational approaches to characterize the cell culture medium were implemented. First, the capacitance and permittivity of the cell culture medium were estimated using a coaxial capacitor. Then, the complex permittivity and conductivity of the cell culture medium were determined using an experimental approach in combination with a computational simulation. Finally, the EFs were computationally calculated.

\subsubsection{Capacitance and permittivity of the cell culture medium $\left(C_{c m}, \varepsilon_{c m}\right)$}

A stainless-steel coaxial capacitor to estimate the cell culture medium capacitance $\left(\mathrm{C}_{\mathrm{cm}}\right)$ was designed and assembled (Figure 5-1A).

A
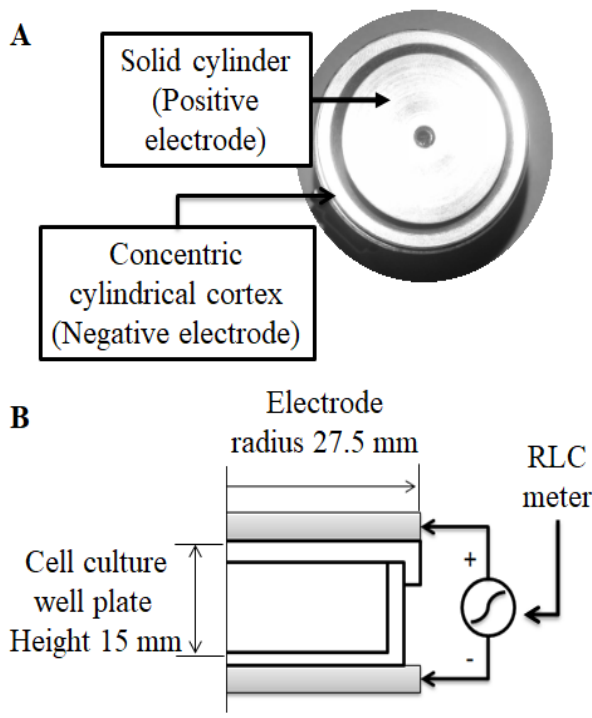
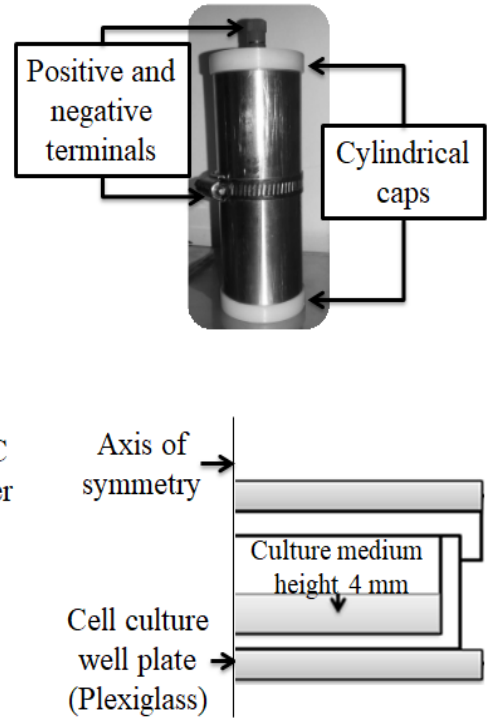

Figure 5-1: Representative scheme of a parallel plate capacitor to determinate the EFs.

A) Coaxial capacitor to estimate the $C_{c m}$. B) Parallel plate capacitor, empty and with cell culture medium. 
The $C_{c m}$ was measured in $40 \mathrm{~mL}$ of culture medium placed between the solid cylinder and the cylindrical cortex. The measurement was performed by connecting the positive and negative terminals to an automatic RLC meter (Fluke 6306 RCL meter, Eindhoven, The Netherlands). Considering that medium change was done every other day for all cultures, changes in the cell culture medium caused by chondrocyte metabolism may be negligible. For this reason, in this study the dielectric constants measurement was made before starting the electrical stimulation and it was taken as representative for the whole culture period.

In addition, the cell culture medium permittivity $\left(\varepsilon_{c m}\right)$ was calculated using the $C_{c m}$ in the Eq. 5-1 [287].

$$
\varepsilon_{m c}=\frac{C_{m c} \ln \left({ }^{r_{o u t}} / r_{i n t}\right)}{2 \pi \varepsilon_{0} L_{c}}
$$

Where $r_{\text {out }}, r_{\text {int }}$ and $L_{c c}$ are the outer radius, the inner radius, and the length of the coaxial capacitor, respectively. Additionally, $\varepsilon_{0}$ refers to the vacuum permittivity.

5.3.2.2 Complex permittivity and conductivity of the cell culture medium $\left(\varepsilon_{j c m}, \sigma_{c m}\right)$

The device used to stimulate cell cultures consists of a parallel capacitive system [155], [156], [162], [167]; however, to our knowledge, the electrical properties of this system either empty or with cell culture medium have not been reported. For this reason, an experimental approach to estimate the resistance $\left(R_{C S}\right)$, capacitance $\left(C_{c S}\right)$, reactance $\left(X_{c S}\right)$ and impedance $\left(Z_{c S}\right)$ of the capacitive system shown in Figure 5-1B was implemented. In this context, the $R_{c S}$ and $C_{c S}$ were found by connecting the electrodes to an automatic RLC meter; the $X_{c s}$ and $Z_{c s}$ were calculated using Eq. 5-2 and Eq. 5-3. In Eq. 5-2 the $f=60 \mathrm{kHz}$ corresponds to the frequency used by different authors to stimulate cell cultures [155]-[158], [161], [162], [167]. 


$$
\begin{gathered}
X_{c s}=\frac{1}{2 \pi f C_{c s}} \\
Z_{c s}=R_{c s}-X_{c s}
\end{gathered}
$$

The $\varepsilon_{j c m}$ and $\sigma_{c m}$ are inherent parameters that characterize a material electrically when it is submitted to EFs. On one hand, the $\varepsilon_{j c m}$ is a constant with both a real and an imaginary part Eq. 5-4, where $\varepsilon_{m c}$ characterizes the capacitive part of the admittance of the capacitor, and $\varepsilon_{j c m}$ characterizes the conductive or loss part of the admittance. On the other hand, the $\sigma_{c m}$ is a property that quantifies the apposition of a material to the current flow. The $\varepsilon_{j c m}$ and $\sigma_{c m}$ were computationally estimated by performing a sensitivity analysis consisting of varying these constants until the $R_{c S}, C_{c S}, X_{c S}$ and $Z_{c S}$ values were satisfied. The electrical simulation of the capacitive system was implemented by a finite element analysis using a software of electromagnetic simulations (COMSOL Multiphysics, Comsol Inc. Los Angeles, USA).

$$
\varepsilon_{j m c}=\varepsilon_{m c}-j \varepsilon_{m c}
$$

Eq. 5-4

\subsubsection{Estimation of EFs}

The EFs were estimated by means of a computational simulation by performing a finite element analysis using COMSOL Multiphysics software. The simulation consisted of modeling the capacitive system shown in Figure 5-1B, by applying a voltage input of 50 and $100 \mathrm{~V}$ at $60 \mathrm{kHz}$ sine wave-form. These two tension values were selected taking into account different literature reports that indicate that those ranges generate EFs capable to stimulate chondrocyte proliferation and molecular synthesis of GAGs [155]-[158], [161], [162], [167]. The dielectric constants of the materials that composed the capacitive system corresponded to those determined in "5.3.2.1" and "5.3.2.2" sections. 


\subsubsection{Electrical stimulation device}

The device designed for the electrical stimulation is composed of two parts (Figure 5-2). The first one consists of stainless-steel electrodes placed in acrylic supports to eliminate any contact with the incubator Surface (Figure 5-2A). In addition, two terminals (positive and negative) were welded to connect the electronic circuit outputs. The second part consists of an electronic circuit that generates the EFs that will stimulate the cell cultures (Figure 5-2B). The electronic circuit is composed of a dual source (Dual source, HM-20, Ceif, National University) that energizes an oscillator to generate tensions of 50 and $100 \mathrm{~V}$ at $60 \mathrm{kHz}$ sine wave-form. Signal verification before and after electrical stimulation was performed using an oscilloscope (Tektronix, TDS 1002, USA).
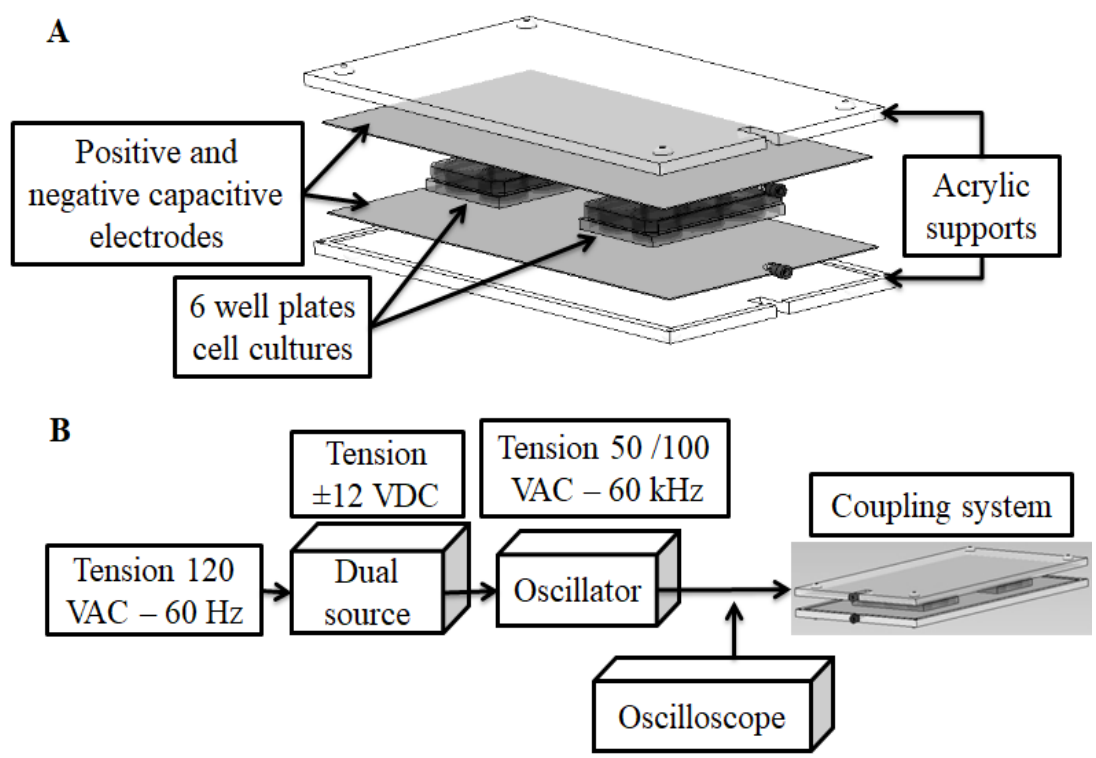

Figure 5-2: Schematic diagram for electrode position and electronic circuit for cell cultures stimulations.

A) Scheme used to homogeneously distribute the EFs in the cell cultures. B) Electronic circuit to generate, distribute and monitor the EFs applied to cell cultures. 


\subsubsection{Electrical stimulation assay}

Stimulated cell cultures were exposed to EFs of 4 and $8 \mathrm{mV} / \mathrm{cm}$ at 60 $\mathrm{kHz}$ sine wave-form. For each of these EFs, three exposure times were tested (30 min, 1 and $5 \mathrm{~h}$ ), and applied daily starting at the second day of culture [156], [161], [162], [167]. Control cell cultures were incubated in the same way as stimulated cell cultures, except that electrodes were not connected to the electronic circuit. Additionally, the top and bottom of the cell culture well plates and electrodes were covered with an electrically conductive gel to eliminate any air gap [156]-[158], [161], [162], [167]. The EFs were applied inside the incubator and the cell cultures remained at $37{ }^{\circ} \mathrm{C}$ and $5 \% \mathrm{CO}_{2}$. In addition, an assay without cells was carried out by applying a continuous $\mathrm{EF}$ of $8 \mathrm{mV} / \mathrm{cm}$ during a period of $5 \mathrm{~h}$ to assess if the EFs affect the $\mathrm{pH}$ within the cell culture medium.

\subsubsection{Proliferation and cell death assay}

Proliferation and cell death were assayed each $48 \mathrm{~h}$. Cells were counted with a hemocytometer and the viability and cell death were determined by Trypan blue stain. Viable cells selectively inhibit Trypan blue to cross the cell membrane; hence, dead cells become blue and can be counted under a light microscope. Final cell density is the number of cells per surface area.

\subsubsection{Glycosaminoglycan quantification}

The quantity of GAGs produced by each cell, in stimulated cell cultures and controls, was estimated in the cell culture medium by a colorimetric assay using 1-9-dimethyl methylene blue, $31 \mu \mathrm{M}$ at $\mathrm{pH}$ 8.0. During the 7 days of stimulation, samples of each well were taken every $48 \mathrm{~h}$, after electrical stimulation, and stored at $20 \mathrm{C}$. All samples were analyzed with a spectrophotometer (BioSpec 1601, Shimadzu) to a $520 \mathrm{~nm}$ wavelength. The quantification was performed using a calibration curve of chondroitin sulfate $\mathrm{B}$, ranging from 0 to $35 \mu \mathrm{g} / \mathrm{mL}$ against reactive blank [288]. 


\subsubsection{Statistical analysis}

All analyses were performed as mean \pm SD $(n=3)$. Cell population growth and GAGs synthesis were analyzed throughout an ANOVA. The model is based on the analysis of individual significance of the data. Before performing the tests, variance homogeneity and normality of data were assessed using the following statistical tests: analysis of variance, test F, test Shapiro-Wilk and Durbin-Watson. The data that did not accomplish these assumptions were analyzed by nonparametric statistics using a test $\mathrm{F}$ and comparing slopes of the proliferation curves. The test $\mathrm{F}$ was also used to analyze cell death. In addition, paired comparisons using the Least Significant Difference test (LSD) were performed. Significant differences were considered at $p<0.05$.

\subsection{Results}

\subsubsection{Cell culture medium characterization and EF estimation}

The $C_{c m}$ and $\varepsilon_{c m}$ listed in Table 5-1 were calculated with a frequency of $60 \mathrm{kHz}$ sine wave-form. The measurement of these constants was performed before and after $48 \mathrm{~h}$ of cell culture post-stimulation in order to ensure that medium capacitance does not vary during stimulation.

Table 5-1: The $C_{m c}$ and $\varepsilon_{m c}$ at $60 \mathrm{kHz}$ sine wave-form.

\begin{tabular}{cccc}
\hline Cell culture medium & $\begin{array}{c}\text { Frequency } \\
(\mathbf{k H z})\end{array}$ & $\mathbf{C}_{\mathbf{m c}}(\mathbf{n f})$ & $\boldsymbol{\varepsilon}_{\mathbf{m c}} *$ \\
\hline DMEM-F12 (before & 60 & $30,66 \pm$ & 402,4 \\
stimulation) & & 0.54 & \\
DMEM-F12 (after 48 h of & 60 & $31.01 \pm$ & 406.8 \\
cell culture post-stimulation) & & 0.66 & \\
\hline
\end{tabular}

The data of $C_{c m}$ were performed as mean $\pm \mathrm{SD}(\mathrm{n}=3)$.

${ }^{*} \varepsilon_{c m}$ was calculated using $C_{c m}$ mean value.

The device used to determine the EF inside the cell culture well plate (Figure 5-2B), allowed to find the resistance and reactance of the empty 
cell culture well plate (Plexiglas) and with cell culture medium. Using these constants, the conductivity and permittivity in empty well plate and with cell culture medium $\left(\sigma_{c m}\right.$ and $\left.\varepsilon_{j c m}\right)$ were computationally determined (Table 5-2). The $\varepsilon_{j c m}$ has a real and complex part; in Table 5-2 the value for each of these variables is presented.

Table 5-2: Electrical constants in vacuum (Plexiglas) and with cell culture medium ( $\sigma_{m c}$ and $\left.\varepsilon_{j m c}\right)$.

\begin{tabular}{cccccc}
\hline & $\begin{array}{c}\text { Resistance } \\
(\mathbf{K} \boldsymbol{\Omega})\end{array}$ & $\begin{array}{c}\text { Reactance } \\
(\mathbf{K} \boldsymbol{\Omega})\end{array}$ & $\begin{array}{c}\text { Impedance } \\
\mathbf{Z}_{\mathbf{0}}-(\mathbf{K} \boldsymbol{\Omega})\end{array}$ & $\begin{array}{c}\text { Conductivity } \\
\boldsymbol{\sigma}-(\mathbf{S} / \mathbf{m})\end{array}$ & $\begin{array}{c}\text { Permittivity } \\
\boldsymbol{\varepsilon}\end{array}$ \\
\hline Plexiglas & 900 & $\mathrm{j} 961,08$ & $900-\mathrm{j} 961,08$ & $6,2 \times 10^{-9}$ & 3,5 \\
DMEM & 751,4 & $\mathrm{j} 796,57$ & $751,4-\mathrm{j} 796,57$ & $200 \times 10^{-3}$ & $402,4-\mathrm{j} 25000$ \\
$\mathbf{F - 1 2}$ & & & &
\end{tabular}

The EF distribution in the whole capacitive system for the empty cell culture well plate and with cell culture medium is shown in Figure 5-3.
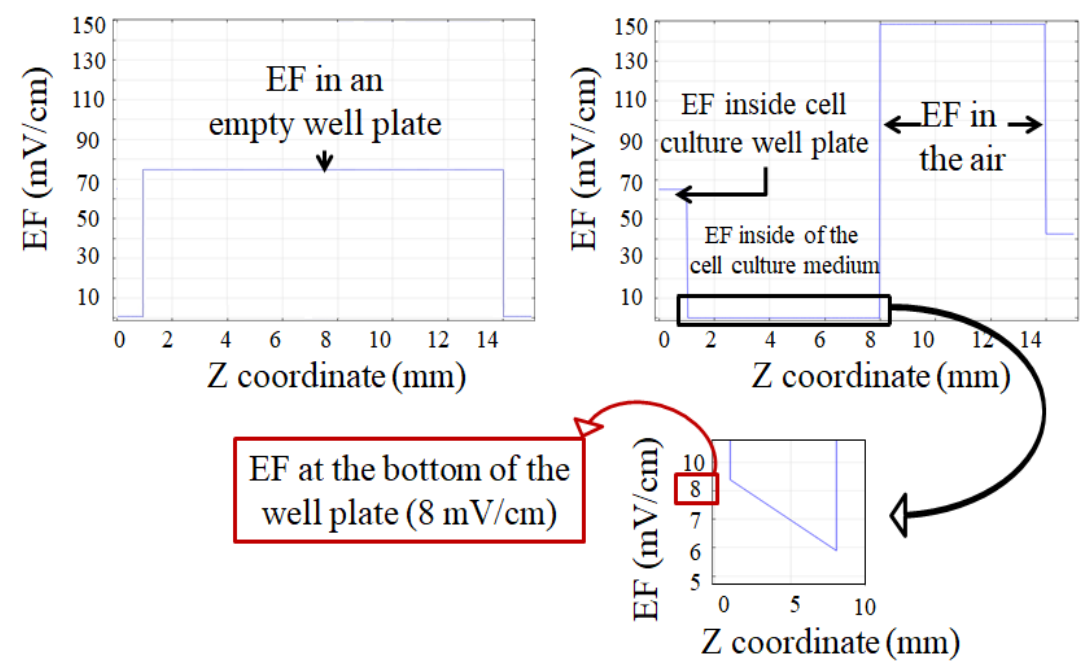

Figure 5-3: Distribution and estimation of the EFs at different heights ( $Z$ coordinate) of the capacitive system.

The voltage applied to the capacitive system to simulate the EF was $100 \mathrm{~V}$ at $60 \mathrm{kHz}$ sine wave form. On the left, the empty capacitive system is illustrated; on the right, the capacitor with cell culture medium is shown. The scheme illustrates the EF decrease due to high dielectric constants $\left(\sigma_{c m}\right.$ and $\left.\varepsilon_{j c m}\right)$. 
Since this simulation integrates the material constants previously obtained, the EF was $4 \mathrm{mV} / \mathrm{cm}$ when a tension of $50 \mathrm{~V}$ was applied; consequently, the EF that stimulates the cell culture with a tension of $100 \mathrm{~V}$ was $8 \mathrm{mV} / \mathrm{cm}$. Note that these EFs values were found within the Z-coordinate of about $1 \mathrm{~mm}$.

\subsubsection{Proliferation and cell death}

Proliferation of stimulated cell cultures and controls showed differences according to applied EF and the scheme of stimulation (Figure 5-4). On one hand, results show an increase in cell population in stimulated cultures, compared with controls, at the fourth and sixth day. This behavior was evidenced in cell cultures that were stimulated with an EF of $4 \mathrm{mV} / \mathrm{cm}$ during $30 \mathrm{~min}$ per day (Figure 5-4A). On the other hand, when EFs of 4 and $8 \mathrm{mV} / \mathrm{cm}$ were applied during $1 \mathrm{~h}$ per day, there were no significant changes in cell population (Figure 5-4B). In contrast, cell culture stimulation using EFs of 4 and $8 \mathrm{mV} / \mathrm{cm}$ during 5 h per day, inhibited cell proliferation completely (Figure 5-4C).

Cell death within the cultures stimulated with EFs of 4 and $8 \mathrm{mV} / \mathrm{cm}$ during $30 \mathrm{~min}, 1 \mathrm{~h}$ and $5 \mathrm{~h}$ did not evidence significant differences compared with controls (Figure 5-5).

\subsubsection{Glycosaminoglycans synthesis}

The amount of GAGs produced by stimulated cell cultures and controls are shown in Figure 5-6. GAGs produced by chondrocytes in monolayer cultures tend to decrease with time. This behavior was observed in cell cultures stimulated with EFs of 4 and $8 \mathrm{mV} / \mathrm{cm}$ during $30 \mathrm{~min}$ and $1 \mathrm{~h}$, as well as in control non-stimulated cultures (Figure 5-6A and Figure 5-6B). However, cell cultures that were stimulated with an $\mathrm{EF}$ of $8 \mathrm{mV} / \mathrm{cm}$ during $5 \mathrm{~h}$ maintained GAGs synthesis through the cell culture period (Figure 5-6C). 

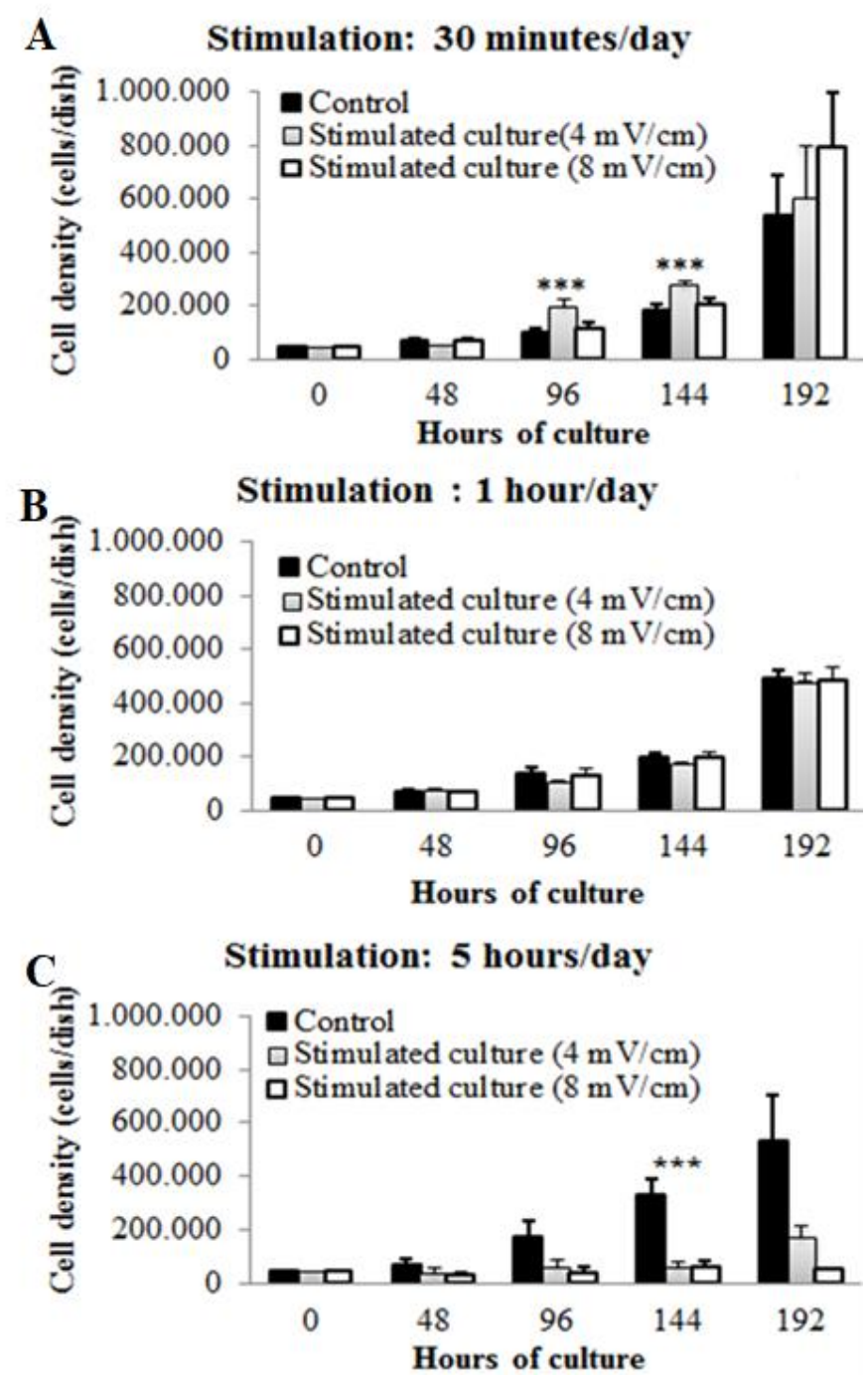

Figure 5-4: Cell population of stimulated and non-stimulated cell cultures.

A) Cell cultures stimulated during 30 min per day vs. controls.

Statistically significant differences were found by applying an EF of 4 $\mathrm{mV} / \mathrm{cm}$ during $30 \mathrm{~min}$ at $96 \mathrm{~h}$ and $144 \mathrm{~h}\left(\mathrm{p}<0.01^{* *}\right)$. B) Cell cultures stimulated during $1 \mathrm{~h}$ per day vs. controls. C) Cell cultures stimulated during 5 h per day vs. controls. Statistically significant differences were found applying an EF of 4 and $8 \mathrm{mV} / \mathrm{cm}$ during $5 \mathrm{~h}$ at $144 \mathrm{~h}$ of culture $(p<0.001 * * *)$. 

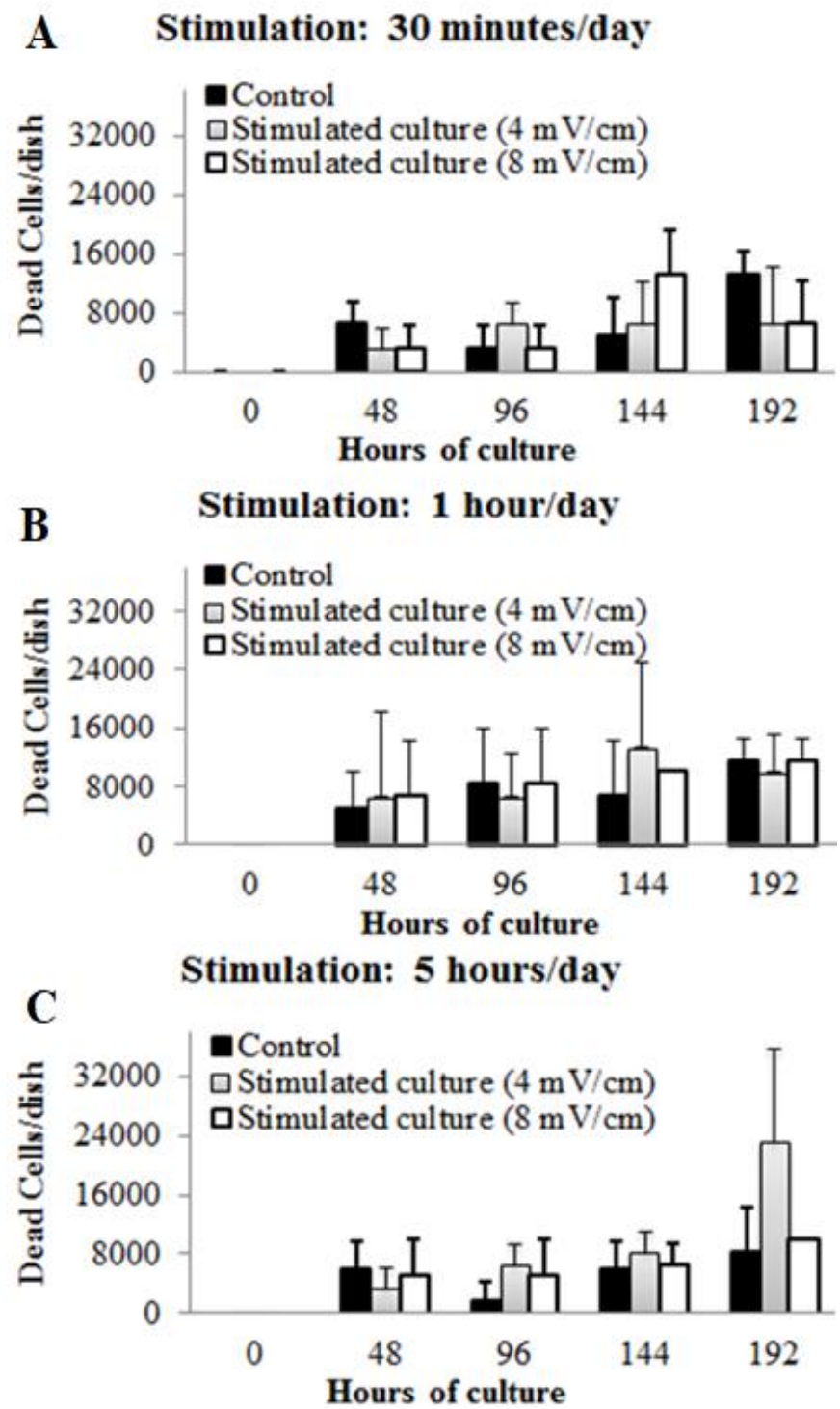

Figure 5-5: Cell death bar graphs for stimulated cell cultures and controls.

A) Cell cultures stimulated during 30 min vs. controls. B) Cell cultures stimulated during $1 \mathrm{~h}$ vs. controls. C) Cell cultures stimulated during $5 \mathrm{~h}$ vs. controls. 

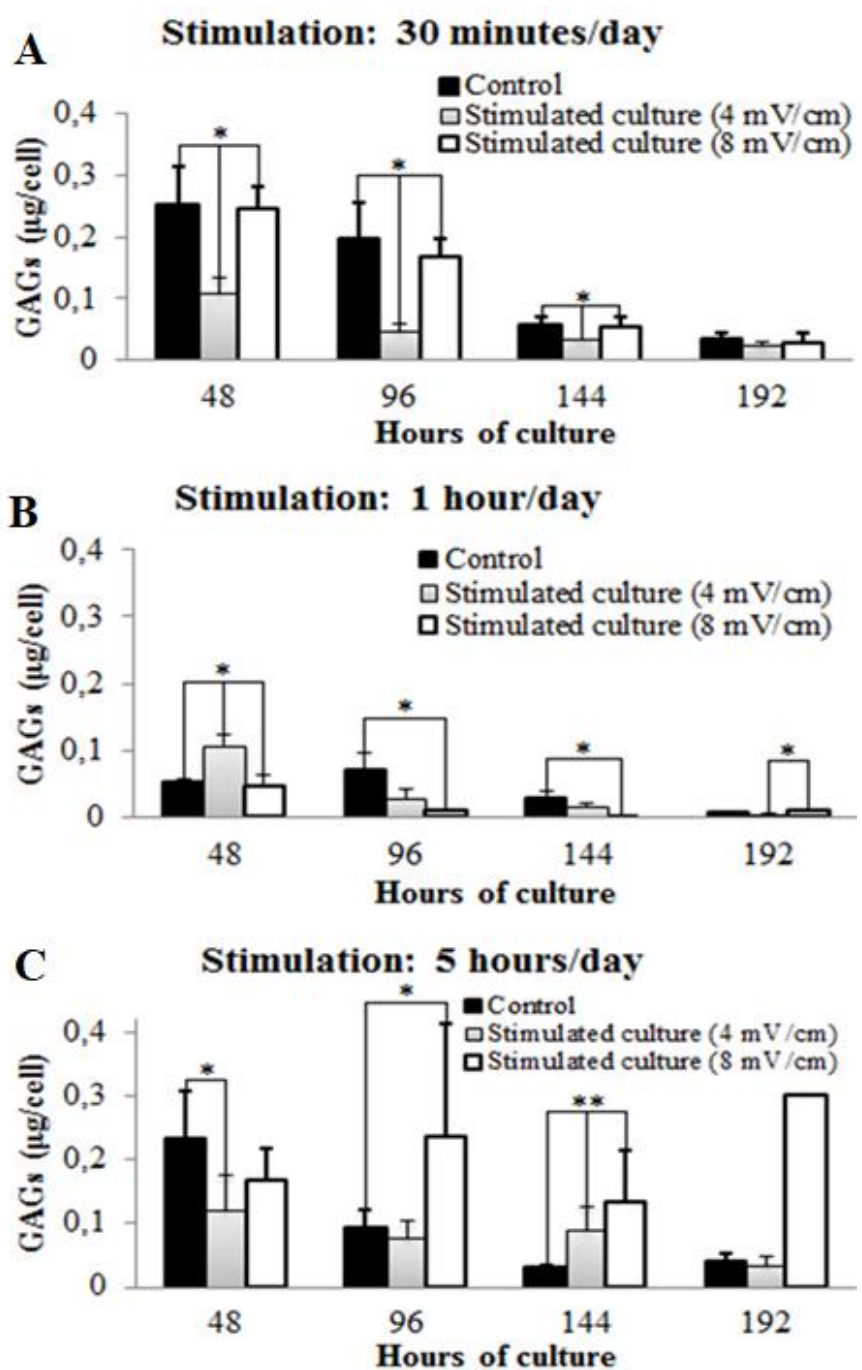

Figure 5-6: Production of GAGs per cell for stimulated cell cultures and controls.

A) Cell cultures stimulated during 30 min vs. controls. Statistically significant differences were found at 48, 96 and $144 h\left(p<0.05^{*}\right)$. B)

Cell cultures stimulated during $1 \mathrm{~h}$ vs. controls. Statistically significant differences were found at $144 h\left(p<0.05^{*}\right)$. C) Cell cultures stimulated during $5 \mathrm{~h} v$ s. controls. Statistically significant differences were found at $48 h\left(p<0.05^{*}\right)$ and $144 h(p<0.01 * *)$. 


\subsection{Discussion}

The aim of this research was to implement a new framework to calculate the EFs that directly stimulate chondrocytes and evaluate the effect over cells cultured in monolayer. Results showed that different EFs applied at different times can increase cell population, inhibit cell proliferative capacity or stimulate GAGs synthesis. On one hand, if the objective of a culture is to obtain a high cell population, an EF of $4 \mathrm{mV} / \mathrm{cm}$ during 30 min may be used as biophysical stimuli to improve cell proliferation. On the other hand, if the aim is to stimulate GAGs synthesis, an EF of $8 \mathrm{mV} / \mathrm{cm}$ during $5 \mathrm{~h}$ ought to be applied. Therefore, an adequate electrical stimulation could be established to optimize the chondrocyte behavior during in vitro cultures. Accordingly, therapies such as the autologous chondrocyte implantation may be enhanced using this methodology.

In order to appropriately establish the electrical stimulation conditions, we characterized the dielectric properties of the cell culture medium. The electrical characterization of cell culture medium included the determination of its conductivity and permittivity. The former is a measure that sets the ability of a material to allow current flow. Based on the conductivity found in this work, it was possible to conclude that the cell culture medium is a conductor material since it showed low electric resistance leading to efficient current flow. In fact, when the cell culture medium is submitted to an EF, a dissociation of positive and negative ions of these salts is generated, allowing a current flow through it. Furthermore, the cell culture permittivity indicates the capacity of a medium to polarize and override the internal EF when an EF is applied to it. Accordingly, the EF applied to the medium was polarized generating a low EF inside cell culture well plate: 4 and $8 \mathrm{mV} / \mathrm{cm}$. In addition, if the permittivity is high, the cell culture medium capacitance increases. In our study, this relation was fulfilled by measuring the cell culture medium capacitance with the coaxial capacitor obtained, indicating that the electric change is restored in an EF with low intensity. 
This research, to our knowledge, is the first attempt to estimate the electrical constants of the cell culture medium, which allow to estimate the EFs inside the cell culture. Thus, the methodology implemented in this study serves as a complement to calculate the EFs in each segment of the stimulation device, such as in the well plate, in the air and in the cell culture medium. In this context, the knowledge generated in this study can be used to calculate the EFs in a more detailed way and compare the works, reported in the literature, that show similarities and discrepancies [156], [157], [161]. One of the limitations of this experimental approach is the difficulty to measure the dielectric constants and subsequently the EFs within the cell culture while cells are being stimulated. Hence, the risk of contamination may increase if an external EF sensor is in contact with the cell culture medium [175]. Since other calculation methodologies or devices to estimate the EF have not been reported in the literature, we propose the computational implementation carried out in this research as a first approach to it.

Regarding the stimulation method, there are two possible schemes to apply the EFs. In the first case, the stimulation may be applied using a direct coupling system in order to assess cell migration and orientation [147], [261], [289]. However, the physicochemical features of the cell culture medium may be altered due to the insufficient biocompatibility of the electrodes that are in contact with the biological material; furthermore, there are potential changes in the $\mathrm{pH}$ and a reduction in the levels of molecular oxygen [175]. Taking such limitation into account, the second method, which consists of an indirect coupling system that uses external parallel electrodes, was implemented in this study. This stimulation method has reported to increase cell population and molecular synthesis in chondrocytes [155]-[159], [161], [162], [167]. The custom-designed device implemented in this research is based on the same principle as used by other authors, which consists of two parallel electrodes connected to a power supply [155], [161], [167]; nevertheless, some improvements to the device were made. For instance, the size of the electrodes was increased in order to assure a homogeneous distribution of the EFs over cultured cells and avoid the edge effect. In addition, an electronic circuit was designed in order to solve the problem of generating high voltages and frequencies between 
the electrodes; therefore, this circuit generates EFs that overcome the limitations caused by the impedance of the well plate and air. According to the changes performed to the device, the results show that it is a useful tool to achieve a biological effect, as evidenced by the changes observed in chondrocyte cell dynamics. In this context, the main advantages of using this device are that it is a noninvasive capacitive coupling system, it is an adaptive system to stimulate any cellular type and it is a device that applies external EFs as it is currently used in medical stimulation. Thus, we proposed a high-performance product that could be used in the industry to modulate chondrocytes behavior.

Experimental analyzes showed that cell behavior is affected by both the EFs intensity and the stimulation time (Table 5-3). An increase in cell proliferation was observed using an EF of $4 \mathrm{mV} / \mathrm{cm}$ during $30 \mathrm{~min}$. This finding is consistent with previous reports [161], [162], [167]. However, if the EF is applied for a longer time $(5 \mathrm{~h})$, cells show inhibition in proliferation since cell population is stable during the whole cell culture period. This finding contrasts with those observed by other authors, who have reported an increase in cell population continuously stimulated for $24 \mathrm{~h}$ [155], [157], [158]. In addition, it was observed that cell death has a similar behavior among the three stimulation schemes; cell death does not increase or decrease in stimulated cultures or controls (Figure 5-5). Based on these findings, it is possible to hypothesize that the applied EFs do not cause cell death.

Table 5-3: Summary of biological effects of EFs applied to cell cultures.

\begin{tabular}{lccc}
\hline & $\begin{array}{c}\text { Stimulation } \\
\text { time/day }\end{array}$ & $\begin{array}{c}\text { EF } \\
(\mathbf{m V} / \mathbf{c m})\end{array}$ & Effect \\
\hline Proliferation & $30 \mathrm{~min}$ & 4 & $\begin{array}{c}\text { Increase at } 96 \text { and } \\
144 \mathrm{~h} \\
\text { GAGs }\end{array}$ \\
& $5 \mathrm{~h}$ & 8 & $\begin{array}{c}\text { Maintenance } \\
\text { during culture }\end{array}$ \\
\hline
\end{tabular}

In terms of molecular synthesis of chondrocytes, in this study, GAGs synthesis to cell culture medium was used as indicator. It was observed that non-stimulated chondrocytes presented a gradual decrease of 
GAGs in medium throughout the culture period. This finding is consistent with a dedifferentiation process that has been reported in chondrocytes cultured in monolayer. This process is characterized by changes in the chondrocytes characteristic round phenotype for an elongated fibroblast-like phenotype, and a decrease in the expression of main characteristic molecules of the articular cartilage [284]. When EFs were applied, chondrocytes GAGs synthesis varied according to the field intensity and exposition time. The stimulation using EFs of 4 $\mathrm{mV} / \mathrm{cm}$ during $30 \mathrm{~min}$ seemed to suppress GAGs synthesis, while cultures exposed to the same field for longer times ( 1 and $5 \mathrm{~h}$ ) showed no significant differences with controls (Figure 5-6C). In contrast, in cultures stimulated using $8 \mathrm{mV} / \mathrm{cm}$ fields we observed that although a scheme of $30 \mathrm{~min}$ per day did not affect GAGs synthesis, later stages of cultures stimulated during 1 h/day or 5 h/day (144 and $192 \mathrm{~h}$ on Figure 5-6C) showed increased GAGs values compared with controls. These results suggest that a long-term exposition of chondrocytes cultures to high EFs favors the preservation of GAGs synthesis. Similar findings have been reported by other authors [155], [157], [158]. Based on the results obtained for the proliferation and GAGs synthesis using the different stimulation schemes, we hypothesize that stimulating a cell culture with high EFs keeps the chondrocytes in a low-proliferative state, maintaining its natural morphology and continuing with the production of molecules of interest. However, further studies using a wider range of EFs from 2 to $10 \mathrm{mV} / \mathrm{cm}$, summed to the analysis of the expression of other molecules are needed to confirm this affirmation, since as observed in our results and some other literature reports, chondrocyte behavior is very sensitive to different electrical stimulation schemes [155], [156]. In addition, there were no changes in the $\mathrm{pH}$ of the cell culture medium; therefore, this variable was not considered when assessing the mechanism of change in cell behavior.

As it was mentioned before, the autologous chondrocyte implantation requires a cellular expansion in a monolayer culture; for this reason, the methodology implemented in this research works under a 2D environment and influences chondrocyte behavior efficiently. In this context, the present study represents an interesting bi-dimensional tool for modulating cell behavior in a normal monolayer culture. The 
methodology used in this work can be extrapolated to perform future models and analyze the effect of EFs over chondrocyte behavior either in scaffolds or within in vivo systems [166], [290]-[293]. It opens a new research area for future works, in which one of the aims would be to calculate the dielectric constants of the 3D-construct and/or tissue and estimate the EFs that directly stimulate either the scaffold or the articular cartilage, since such variables may differ from those found in our in vitro model.

\subsection{Concluding Remarks}

This research evidences changes in cell dynamics when chondrocytes are stimulated with different EF intensities and exposure times. Within this context, depending on the EFs and time of stimulation, in vitro procedures can be focused either on increasing cell population or enhancing synthesis of GAGs. Additionally, in this research a new methodology to calculate and apply EFs to cell cultures was implemented by designing a specialized device and using computational simulation. Furthermore, the procedures carried out in this research are suitable to be extrapolated to study cell behavior in different cell types, representing a new way in tissue engineering and regenerative medicine research. 


\title{
CHAPTER 6
}

\author{
Capacitively coupled \\ electrical stimulation of rat \\ chondroepiphysis explants
}





\subsection{Summary ${ }^{5}$}

The growth plate is a cartilaginous layer present from the gestation period until the end of puberty where it ossifies joining the diaphysis and epiphysis. During this period several endocrine, autocrine, and paracrine processes within the growth plate are carried out by chondrocytes; therefore, a disruption in cellular functions may lead to pathologies affecting bone development. It is known that electric fields impact the growth plate; however, parameters such as stimulation time and electric field intensity are not well documented. Accordingly, this study presents a histomorphometrical framework to assess the effect of electric fields on chondroepiphysis explants. Bones were stimulated with 3.5 and $7 \mathrm{mV} / \mathrm{cm}$, and for each electric field two exposure times were tested for 30 days ( $30 \mathrm{~min}$ and $1 \mathrm{~h}$ ). Results evidenced that electric fields increased the hypertrophic zones compared with controls. In addition, a stimulation of $3.5 \mathrm{mV} / \mathrm{cm}$ applied for $1 \mathrm{~h}$ preserved the columnar cell density and its orientation. Moreover, a pre-hypertrophy differentiation in the center of the chondroepiphysis was observed when explants were stimulated during $1 \mathrm{~h}$, with both electric fields. These findings allow the understanding of the effect of electrical stimulation over growth plate organization and how the stimulation modifies chondrocytes morphophysiology.

\subsection{Introduction}

The growth plate also known as epiphyseal plate is a cartilaginous tissue located between the epiphysis and the diaphysis, and it is responsible for the longitudinal growth and shape of long bones [7], [8]. Given its location, the epiphyseal plate is exposed to external stimuli that generate different signals involved in the physiological regulation of cell behavior and various pathological problems. The response of chondrocytes to biophysical stimuli results in the synthesis of the

\footnotetext{
${ }^{5}$ The results presented in this chapter has been published in: J.J. Vaca-González, J.F. Escobar, J.M. Guevara, Y.A. Hata, G. Gallego Ferrer and D.A. Garzón-Alvarado, "Capacitively Coupled Electrical Stimulation of Rat Chondroepiphysis Explants: A Histomorphometric Analysis”. Bioelectrochemistry, 126, 1-11, 2019.
} 
molecules that compose the extracellular matrix (ECM) of the growth plate, such as proteins (collagen type II and X, Ihh, BMP, WNT), proteoglycans (glycosaminoglycans (GAGs), aggrecan) and growth factors (VEGF, TGF $\beta$, PTHrP) [16], [294]. Histologically, the growth plate is stratified in different zones according to the phenotype of the chondrocytes and the spatial arrangement of the ECM. Consequently, the growth plate is organized into four zones: reserve, proliferative (P$\mathrm{z}$ ), pre-hypertrophic (PH-z) and hypertrophic (Ht-z) [15]. Other morphological characteristics of the growth plate are the shape and width that change according to the species, the type of bone and age. For example, it has been shown that the morphology of the growth plate in the proximal femoral epiphysis of humans is concave at the age of four years, straight at the age of seven and convex at the beginning of the puberty [17]. Regarding the width of the growth plate, the physis is wider in early stages of development; while at the end of the adolescence the thickness of the physis decreases progressively, until it is completely reabsorbed by connecting the epiphysis and the metaphysis in a process known as epiphyseal fusion [16]. On the contrary, the growth plates in mammals vary according to the location within the bone. For example, the epiphyseal plate in the proximal femur is concave in the postnatal stages, while in the distal femur it has an irregular morphology. This architecture tends to be constant during bone growth; nevertheless, some changes can be observed 21 days after birth when the growth plate becomes thicker and the distal physis acquires a wavy morphology. Unlike the growth plate in humans, where complete ossification occurs once sexual maturity is reached, the epiphyseal plate is preserved throughout life in rodents [295].

The growth plate is an avascular and aneural tissue that has limited selfhealing properties due to its poor vascularization, reduced migration and mitogenic characteristics of chondrocytes [5], [139]. Therefore, a damage in the ECM and/or chondrocyte death may result in dysfunction of the epiphyseal plate and subsequent defects in bone development [45], [196]. Considering that growth plate responds to external biophysical stimuli, studies have shown that compressive mechanical loading induces to histological changes at cellular level within the growth plate, specifically in the width of the P-Z and Ht-z, as well as 
disruption of columnar arrangement [197], [200], [204]. The effects of compressive loading within the tissue include chondrocyte deformations, changes in the interactions among collagens and proteoglycans present in the ECM, and flux of water. The latter has been associated to the application of other types of biophysical stimuli such as tension, shear stress and hydrostatic pressure that alter the molecular activity of proteases and their inhibitors (e.g. TIMPs, MMPs), soluble factors, pro-inflammatory factors (e.g. Nitric Oxide, Prostaglandin E, Cyclooxygenase 2) and proteoglycans (e.g. GAGs) [240], [245], [256]. Although mechanical stimuli play a relevant role in growth plate development, it is difficult to extrapolate final conclusions regarding characteristics of the stimuli and cell response, considering that there is a high variability in terms of strain magnitude, loading duration and frequency. In fact, it has been described that cell deformations caused either by changes in cell volume or due to biophysical stimuli, lead to the activation of different types of ionic channels, such as intracellular calcium levels [260], [268], [296]-[298]. Similarly, other type of stimuli such as electric fields (EFs) may stimulate the ion channels of the chondrocyte cell membrane and control, extra and intracellularly, the pathway-signaling [260], [268]. It has been evidenced that EFs have an effect over growth plates zones altering mainly its morphology. For instance, an increase in length and width of distal femurs was observed when EFs stimulated the growth of the proliferative zone making it wider with a well-stratified columnar arrangement [180]. A similar study showed that EFs caused a mild proliferation of the hypertrophic zone towards the marrow cavity in the central area of the growth plate [182]. External devices have been also used to apply EFs and it has been evidenced that this electrical stimulus reduces bone growth, maintains cartilage in a quiescent state and promotes weight loss in animals without changes in bone growth [183], [299].

Even though in vivo electrical assays have evaluated the changes in the morphology of the growth plate and the development of long bones, there are many discrepancies in the results obtained; moreover, there are no studies that explore in detail the underlying effects that the EFs have over growth plate morphology and chondrocyte behavior. The 
main reason of this inconsistency is because factors such as EFs magnitudes, stimulation time per day, and the amount of days that the growth plate need to be under stimulation have not been well documented. Moreover, a morphological study has not been carried out to observe how the EFs alter the morphology of the growth plate zones. According to this, chondroepiphysis explants of femur and humerus were stimulated with 7 and $3.5 \mathrm{mV} / \mathrm{cm}$ at $60 \mathrm{kHz}$ (sine wave-form) using two different stimulation schemes (30 min and $1 \mathrm{~h}$ ). These exposure times were applied four times per day during 30 days of culture. To accomplish this, a stimulation scheme, based on a capacitively coupling system, was designed and implemented to generate and homogeneously distribute the EFs over the explant cultures. Parameters such as frequency, duration and effective stimulation period to stimulate explants are based on previous findings, which have evidenced that EFs of lower intensity at $60 \mathrm{kHz}$ stimulate chondrocyte dynamics, specially cell proliferation and molecular expression [159], [161], [178]. Moreover, it was evidenced that EFs of 16.5 and $33.5 \mathrm{mV} / \mathrm{cm}$ at $60 \mathrm{kHz}$ have little effect on composition of the growth plate [180], but can alter the thickness of zones within the physis [180], [182], and the length of the bone [180], [181]. Results from this study indicated that depending on the EFs and the scheme used to stimulate the explants, the growth plate zones change in terms of width, cell arrangement and cell morphology. In addition, it was observed that EFs induce chondrocytes to enter in a pre-hypertrophy state in the center of the epiphysis. These findings are relevant since non-invasive therapies such as electrostimulation can be improved to restore pathological tissues. In fact, electrical stimulation of in vitro cultures may be used to understand how cell dynamics interact within the tissue and trigger certain molecular events that modulate tissue growth. This optimization brings a better understanding of long bone development and new therapeutic strategies for future cartilage tissue engineering and regenerative medicine. 


\subsection{Materials and methods}

\subsubsection{Chondroepiphysis isolation and in vitro culture}

2-day-old Wistar rats $(\mathrm{n}=20)$ obtained from the Veterinary Medicine Faculty of Universidad Nacional de Colombia were used in this study. The rats were sacrificed by decapitation at the Biomimetics Laboratory (Biotechnology Institute in Universidad Nacional of Colombia). Recommendations from the ethics committee were followed for this experimental procedure. First, femurs and humerus were isolated and washed with PBS containing 2\% of antibiotics (streptomycin and penicillin, LONZA, Walkersville, MD USA). Next, the fibrous tissue and muscles were carefully removed from the epiphyses and the diaphyses. Just one epiphysis per bone was electrically stimulated in this study; therefore, the proximal epiphysis was removed from the femur, while the distal epiphysis was detached from the humerus. A large portion of the diaphysis of the bone was also extracted to preserve the ossification front of the growth plate. The extremities were fixed vertically into a 24 well-plate in order to apply the EFs perpendicularly to the chondroepiphysis (Figure 6-1A). Bone fixation was performed with an agarose disc located at the bottom of the well-plate (agarose at $3 \%$ diluted in distilled water). Once the explants were located vertically, $2 \mathrm{~mL}$ of culture medium were added (DMEM-F12 with $\beta$-Glicerol phosphate $1 \mathrm{mM}$, ascorbic acid $50 \mu \mathrm{g} / \mathrm{mL}$, Fetal Bovine Serum (FBS) $0.2 \%$, and antibiotics $1 \%$ ). The explants were cultured at $37{ }^{\circ} \mathrm{C}$ and $5 \%$ $\mathrm{CO}_{2}$ atmosphere. Culture medium was changed every other day. This procedure was implemented for controls and stimulated cultures

\subsubsection{Electric field estimation}

A computational simulation was implemented to estimate the EFs within the capacitively coupling system. The EFs were generated using voltages of 100 and $200 \mathrm{Vpp}$ at $60 \mathrm{kHz}$ (Figure 6-1A). The capacitively coupling system was modelled as an axisymmetric configuration to calculate the EFs values in each part of the culture. The dielectric properties of the different materials that composed the capacitive system were considered (Table 6-1). The agarose disc was not taken 
into account in the computational simulation due to the fact that the EF in this zone is negligible. A single well-plate from a 24 well-plate was simulated and the electrodes were big enough at the top and bottom of the well to ensure a homogeneous and isotropic distribution of the $\mathrm{EF}$ during the simulation (Figure 6-1B). The computational simulation was implemented by a finite element analysis using an electromagnetic simulations software (COMSOL Multiphysics, Comsol Inc. Los Angeles, CA USA).

A

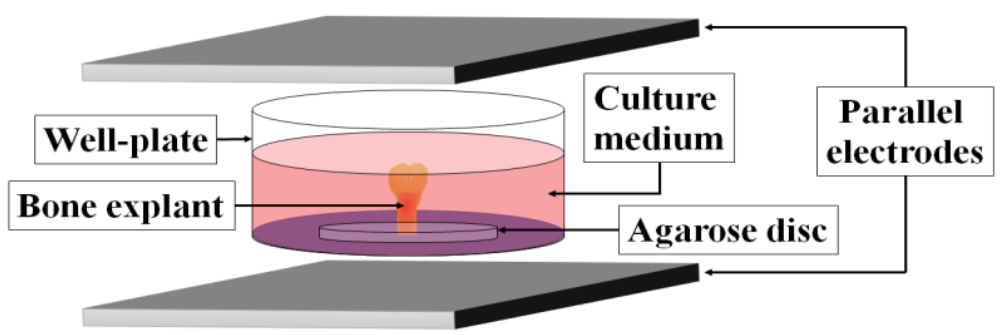

B

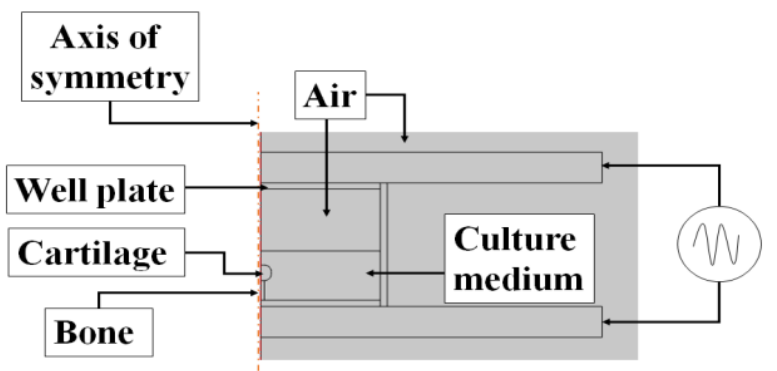

Figure 6-1: Capacitively coupling electrical system.

A) Fixation of the explants using an agarose disc into the bottom of a single well. B) Axisymmetric system used for the computational simulation.

\subsubsection{Electrical stimulation assay}

Electrical stimulation was performed using a coupled system that delivered homogeneous EFs (Figure 6-2) [178]. The electrical stimulation device was composed of two parallel stainless-steel electrodes located at the top and bottom of the culture well-plates 
(Figure 6-2A). The electrodes were placed in Teflon supports to eliminate any contact with the metallic surface of the incubator. The positive and negative terminals of the electrodes were connected to an electronic circuit (oscillator) that generated the voltages and frequencies required to create the EFs. The oscillator was energized with a dual source (Lendher - HY3003D-3, Shenzhen, China) and the signal verification before and after electrical stimulation was monitored using an oscilloscope (Keysight - DSO1052B, Santa Rosa, CA, USA). Control explant cultures were incubated in the same way as stimulated explant cultures, except that electrodes were not connected to the oscillator.

Table 6-1. Dielectric properties and measurements used in the simulation.

\begin{tabular}{|c|c|c|c|}
\hline Component & Parameter & $\begin{array}{l}\text { Value used in } \\
\text { simulation }\end{array}$ & References \\
\hline \multirow{5}{*}{$\begin{array}{l}\text { Stainless- } \\
\text { steel }\end{array}$} & Electrode separation & $20[\mathrm{~mm}]$ & \\
\hline & Electrode radius & $50[\mathrm{~mm}]$ & \\
\hline & Thickness & $5[\mathrm{~mm}]$ & \\
\hline & Relative permittivity & 1 & \\
\hline & Electric conductivity & $1.73913[\mathrm{MS} / \mathrm{m}]$ & \\
\hline \multirow{3}{*}{$\begin{array}{l}\text { Bone } \\
\text { marrow }\end{array}$} & Length and thickness & 3.2 and $0.5[\mathrm{~mm}]$ & \multirow{3}{*}[300]{} \\
\hline & Relative permittivity & 102.8 & \\
\hline & Electric conductivity & $0.002901[\mathrm{~S} / \mathrm{m}]$ & \\
\hline \multirow{3}{*}{ Cartilage } & Length and thickness & 2.5 and $1.5[\mathrm{~mm}]$ & \multirow{3}{*}{$\begin{array}{l}{[300],} \\
{[301]}\end{array}$} \\
\hline & Relative permittivity & 2706.5 & \\
\hline & Electric conductivity & $0.17732[\mathrm{~S} / \mathrm{m}]$ & \\
\hline \multirow{3}{*}{$\begin{array}{l}\text { Culture } \\
\text { medium }\end{array}$} & Height & $8[\mathrm{~mm}]$ & \multirow{3}{*}[302]{} \\
\hline & Relative permittivity & $402.4-\mathrm{j} 25000$ & \\
\hline & Electric conductivity & $0.0834[\mathrm{~S} / \mathrm{m}]$ & \\
\hline \multirow{4}{*}{ Well-plate } & Length and height & 17.5 and $20[\mathrm{~mm}]$ & \multirow{6}{*}{ [302] } \\
\hline & Thickness & $1[\mathrm{~mm}]$ & \\
\hline & Relative permittivity & 3.5 & \\
\hline & Electric conductivity & $6.9[\mathrm{nS} / \mathrm{m}]$ & \\
\hline \multirow{2}{*}{ Air } & Relative permittivity & 1 & \\
\hline & Electric conductivity & $0[\mathrm{~S} / \mathrm{m}]$ & \\
\hline
\end{tabular}


Stimulated cell cultures were exposed to EFs of 3.5 and $7 \mathrm{mV} / \mathrm{cm}$ at 60 $\mathrm{kHz}$ sine wave-form during 15 and 30 days of culture. The EFs were applied the first day of the isolation using two exposure times (30 min and $1 \mathrm{~h}$ ), according to a previous report [178]. Each stimulation time was applied four times per day according to the following scheme: 30 min of stimulation corresponds to $5.5 \mathrm{~h}$ without stimulation, while $1 \mathrm{~h}$ of stimulation corresponds to $5 \mathrm{~h}$ without stimulation. A schematic description of the signal protocol is given in Figure 6-2B. A temperature control assay was performed when explants were submitted to continuous EFs of $7 \mathrm{mV} / \mathrm{cm}$ during a period of $1 \mathrm{~h}$.

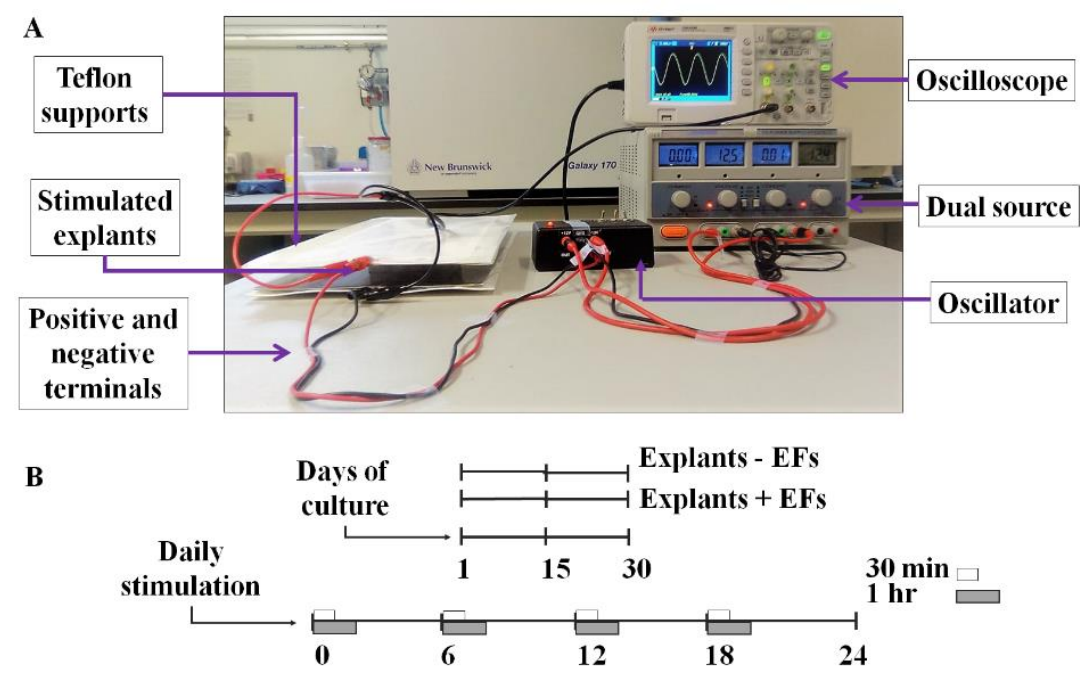

Figure 6-2. Schematic diagram of the in vitro stimulation.

A) Capacitively coupling electrical stimulation in vitro. Connection of the dual source, oscillator and oscilloscope to the parallel electrodes.

B) Diagram of the experimental design. Explants were randomly divided into 10 groups according to culture days, EFs and stimulation period: (1) 15 days $-3.5 \mathrm{mV} / \mathrm{cm}-30 \mathrm{~min}$, (2) 15 days $-7 \mathrm{mV} / \mathrm{cm}-30$ min, (3) 30 days $-3.5 \mathrm{mV} / \mathrm{cm}-30 \mathrm{~min}$, (4) 30 days $-7 \mathrm{mV} / \mathrm{cm}-30$ $\mathrm{min}$, (5) 15 days $-3.5 \mathrm{mV} / \mathrm{cm}-1 \mathrm{~h}$, (6) 15 days $-7 \mathrm{mV} / \mathrm{cm}-1 \mathrm{~h}$, (7) 30 days $-3.5 \mathrm{mV} / \mathrm{cm}-1 \mathrm{~h}$, (8) 30 days $-7 \mathrm{mV} / \mathrm{cm}-1 \mathrm{~h}$, (9) 15 days without stimulation and (10) 30 days - without stimulation. 


\subsubsection{Growth plate histological analysis}

\subsubsection{Masson's trichrome staining}

Control and stimulated explants were stained with Masson's trichrome technique to observe chondrocyte nucleus, cytoplasm and collagen fibers in the chondroepiphysis. All samples were fixed in formaldehyde at $4 \%$ for $24 \mathrm{~h}$ at room temperature. Then, explants were embedded in paraffin and sectioned at $5 \mu \mathrm{m}$ for Masson's trichrome staining. An imaging analysis of growth plates were performed using a microscope with integrated camera (Nikon Eclipse E600W, Melville, NY USA) at two different magnifications (4X and 10X).

\subsubsection{Quantitative analysis of growth plate zones}

Considering the protocols described by [303] to analyze growth plates, some histomorphometric parameters were measured to quantify the size of P-z, PH-z and Ht-z, the total length of the epiphyseal plate, and the columnar organization in each growth plate zone (Figure 6-3A). For measuring zonal size (P-z, PH-z and Ht-z), the different zones were delimited manually in 10X-magnification images according to cell morphology. A minimum of 90 measures were obtained for each growth plate zone through evaluation of at least 3 images per explant (1 image $\mathrm{x} 3$ independent sections). Then, ten parallel lines (green) were drawn in the direction of longitudinal bone growth to have different measures of each zone (Figure 6-3B). The size of the measured zone corresponded to the average length of each parallel line from the top to the bottom of each zone, which was separated by the hand-made lines. The total size of the growth plate was the result of the sum of the P-z, $\mathrm{PH}-\mathrm{z}$ and Ht-z lengths. The quantification of chondrocytes and columns in each zone was performed by drawing three meshes (yellow grids) over 10X-magnification images (Figure 6-3C). The square size varied for each zone corresponding to 36x36 $\mu \mathrm{m}$ squares for P-z and PH-z, and 100x $100 \mu \mathrm{m}$ squares for Ht-z. Using these grids, three parameters were measured: density of isolated cells $\left(C_{i}\right)$, columnar cells $\left(C_{c}\right)$ and column density $\left(C_{D}\right)$. Moreover, a cartesian axis was drawn into the meshes to 
measure the inclination angle $(\alpha)$ of the columns in all zones (Figure 6-3D). Here, a cell column was defined as three or more stacked cells with a distance among them not greater than the average cell height within the field. All images were processed with Image Fiji Software and at least 3 images per individual (1 image $\mathrm{x} 3$ independent sections) were analyzed. (NIH Image software, Bethesda, MD USA).

A

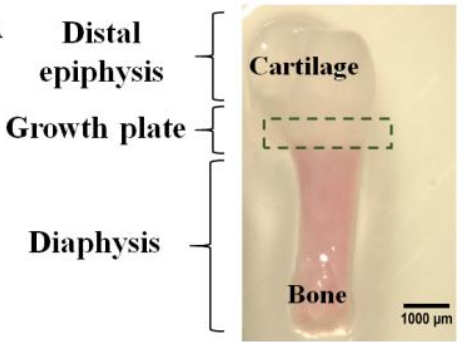

C

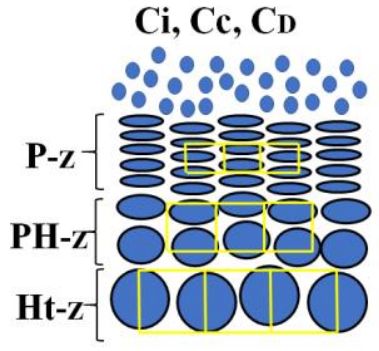

B

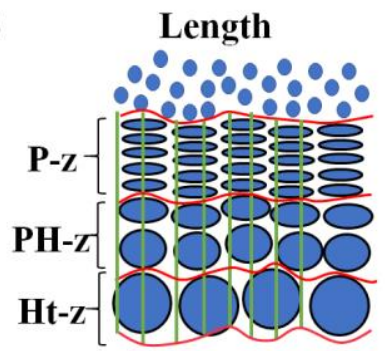

D

\section{Columns angle}
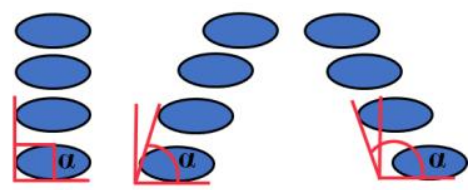

Figure 6-3. Representative diagram for the quantitative analysis of growth plate zones.

A) Representation of explant bone used for cultures and its parts. B) Scheme that shows the treatment applied to the growth plate images to measure the height of the $\mathrm{P}-\mathrm{z}, \mathrm{PH}-\mathrm{z}$ and $\mathrm{Ht}-\mathrm{z}$. C) Scheme that shows the treatment performed to the epiphyseal plate images to quantify the chondrocytes in each zone. D) Columnar arrangement and orientation.

\subsubsection{Statistical analysis}

For the statistical studies, all analyses were performed with mean \pm SD $(n=3)$ according to the protocol described by [304]. Both a one-way ANOVA, and a Least Significant Difference (LSD) - Fisher post-test were carried out to analyze the histomorphometric parameters of the 
growth plate. Moreover, a Kruskal-Wallis nonparametric test or an unpaired two-tailed t-test were performed where applicable. Finally, a multifactorial ANOVA was done to observe which factors such as EFs, stimulation time or time culture have an effect over growth plate morphology and cell behavior. Statgraphics Centurion software was used for the statistical analysis (Statgraphics Technologies, Inc. The Plains, VA USA).

\subsection{Results}

\subsubsection{Estimation of EFs}

The EF estimation in the capacitively coupling system for a single wellplate is shown in Figure 6-4. This simulation was performed applying a voltage of $100 \mathrm{Vpp}$ at $60 \mathrm{kHz}$ sine wave-form between the electrodes. The EF distribution in the capacitive system for the empty well-plate and with culture medium is shown in Figure 6-4A. Measurements in five zones of the capacity system were performed to show the EFs in detail (Figure 6-4B). Results evidenced that in zone $a$ (blue arrow in Figure 6-4B) exists a constant $\mathrm{EF}$ of $1.08 \times 10^{5} \mathrm{mV} / \mathrm{cm}$ within the empty well-plate (Figure 6-4C left side), while in zone $a^{\prime}$ (blue arrow in Figure 6-4B) the EFs within the well-plate with bone explants and culture medium vary depending on where it is measured (Figure 6-4C right side).

The EF values in the capacitive system are shown in Figure 6-5. In zone $b$ (red arrow in Figure 6-4B) there is an EF of $7 \mathrm{mV} / \mathrm{cm}$ in the center of the chondroepiphysis (Figure 6-5A), while in zone $c$ (yellow arrow in Figure 6-4B) there are EFs from $13.5 \mathrm{mV} / \mathrm{cm}$ in the bottom of the well-plate to $7.5 \mathrm{mV} / \mathrm{cm}$ at the surface of the culture media (Figure 6-5B). In zone $d$ (cyan arrow in Figure 6-4B) there is an EF of $1.8 \times 10^{5}$ $\mathrm{mV} / \mathrm{cm}$ in the air gap (Figure 6-5C). Finally, in zone $e$ (green arrow in Figure 6-4B) there is an EF of $1.03 \times 10^{5} \mathrm{mV} / \mathrm{cm}$ outside the well-plate between the electrodes (Figure 6-5D). As two EF intensities were tested in this study, the EFs generated by a voltage of $50 \mathrm{Vpp}$ at $60 \mathrm{kHz}$ sine wave-form are shown in the Appendix F and Appendix G. Due to 
the fact that EFs have a lineal increment, the intensities of EFs when applying $50 \mathrm{Vpp}$ are half of those mentioned above.

A

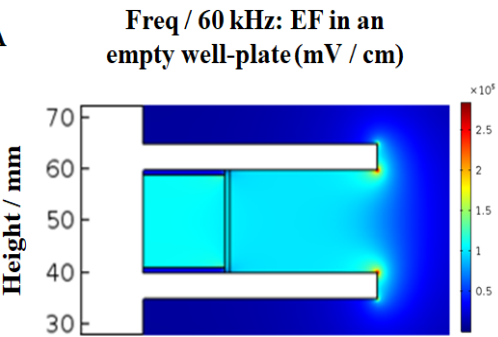

B

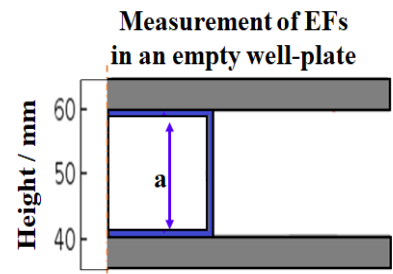

C

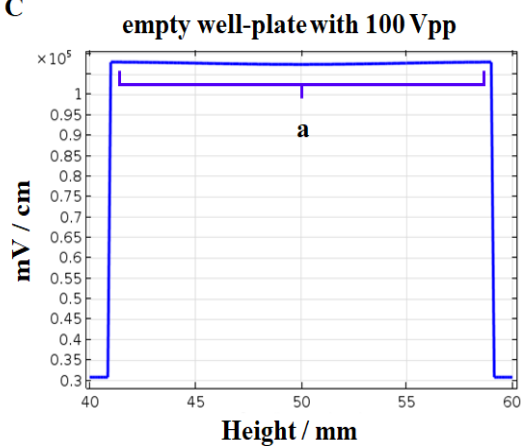

Freq / $60 \mathrm{kHz}$ : EF in a well-plate with bone explant and culture medium $(\mathrm{mV} / \mathrm{cm})$

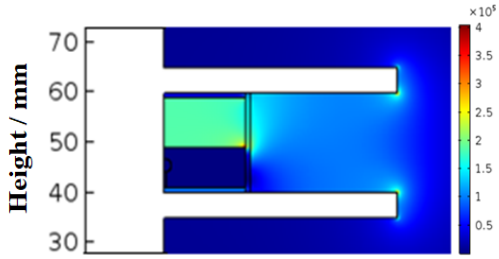

Measurement of EFs in well-plate with explants and culture medium

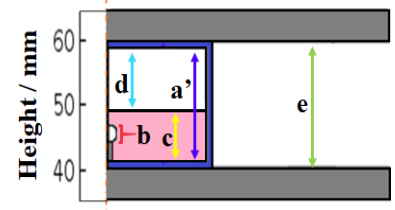

Distribution of the EF inside the well-plate with $100 \mathrm{Vpp}$

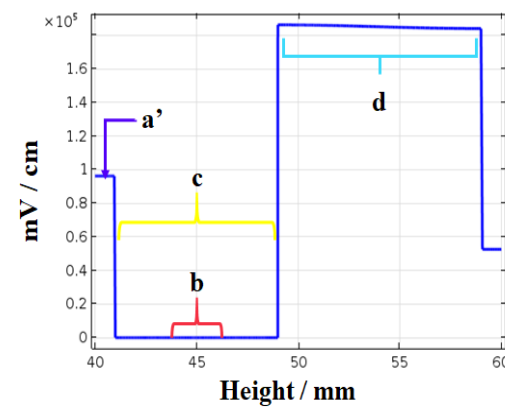

Figure 6-4. Representative scheme of the EFs in the capacitively coupling system generated by a voltage $100 \mathrm{Vpp}$ at $60 \mathrm{kHz}$ sine wave-form.

A) EFs in an empty capacitive system and with bone explants and culture medium. B) Measurement points of the EFs within and outside the well-plate. C) On the left, the EF in the empty capacitive system is illustrated; on the right, the EF distribution in the capacitor with the explant bone and culture medium is shown. 
A

Distribution of the EF inside the chondroepiphysis with $100 \mathrm{Vpp}$
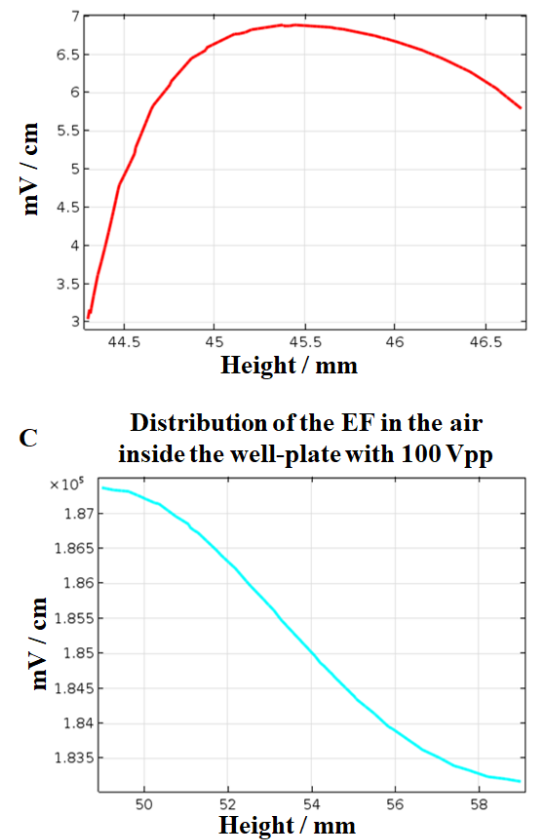

B

Distribution of the EF in the culture medium with $100 \mathrm{Vpp}$
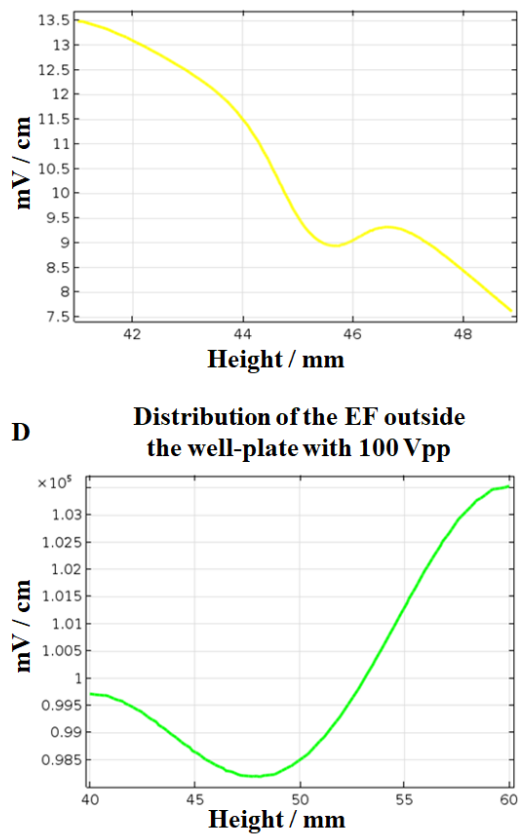

Figure 6-5. EF intensities within the capacitively coupled system applying a voltage $100 \mathrm{Vpp}$ at $60 \mathrm{kHz}$ sine wave-form.

A) EF within the chondroepiphysis. B) The EF in the culture medium.

$C)$ The EF in the air gap between the culture medium and the top of the well-plate. D) The EF outside the well-plate between the electrodes.

\subsubsection{Histomorphometric of the growth plate}

\subsubsection{Thickness and morphology of growth plate zones}

The histomorphometric measurements of the growth plate width and the thickness of each zone in femur explants are shown in Figure 6-6. The epiphyseal plate width of control bones decreased from D15 to D30, while the epiphyseal plate experienced a different behavior depending on the EFs applied and culture time. For instance, the width of femur explants stimulated either with 3.5 or $7 \mathrm{mV} / \mathrm{cm}$ for 15 days did not change, while those stimulated for 30 days tended to be thinner, 
excepting the explants that were stimulated with $3.5 \mathrm{mV} / \mathrm{cm}$ for $30 \mathrm{~min}$ and $7 \mathrm{mV} / \mathrm{cm}$ for $1 \mathrm{~h}$ where the width remained stable (Figure 6-6A).

Histologically, control bones showed highly proliferative flattened chondrocytes arranged into columns in the P-z, followed by prehypertrophic chondrocytes in the PH-z. Finally, chondrocytes that had stopped cell division and increased their size turned into hypertrophic in the Ht-z (Figure 6-7A). Thickness and cell morphology of growth plate zones in stimulated explants vary according to the EFs applied. For example, the P-z tends to disappear during culture in both controls and stimulated cultures (Figure 6-6B).

A

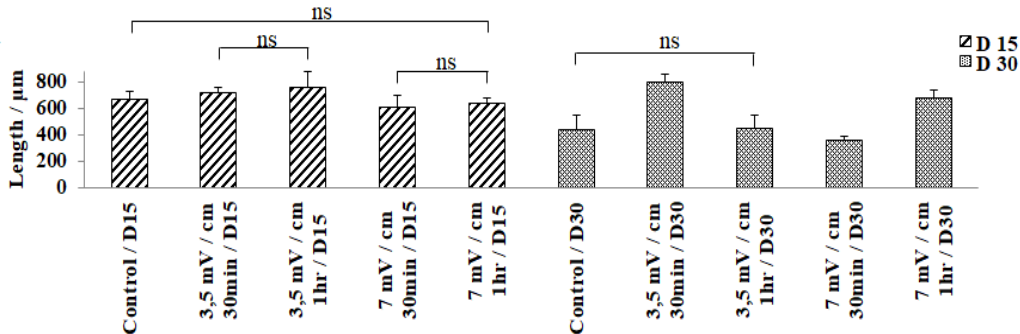

B

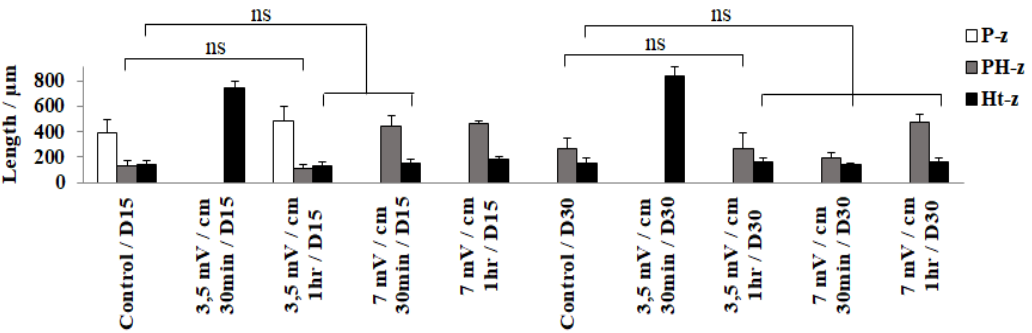

Figure 6-6. Measurement of growth plate lengths and thickness of $\mathrm{P}-\mathrm{z}, \mathrm{PH}-\mathrm{z}$ and $\mathrm{H} t-\mathrm{z}$.

A) Length of distal femur growth plates. B) Thickness of distal femur epiphyseal plate zones. Statistically significant differences were found between controls and stimulated explants in terms of length and thickness after a stimulation with EFs of 3.5 and $7.5 \mathrm{mV} / \mathrm{cm}$ ( $p<$ $0,05)$, excepting those marked with " $n s$ " (not significant). 
Chapter 6. Electrical stimulation of chondroepiphysis
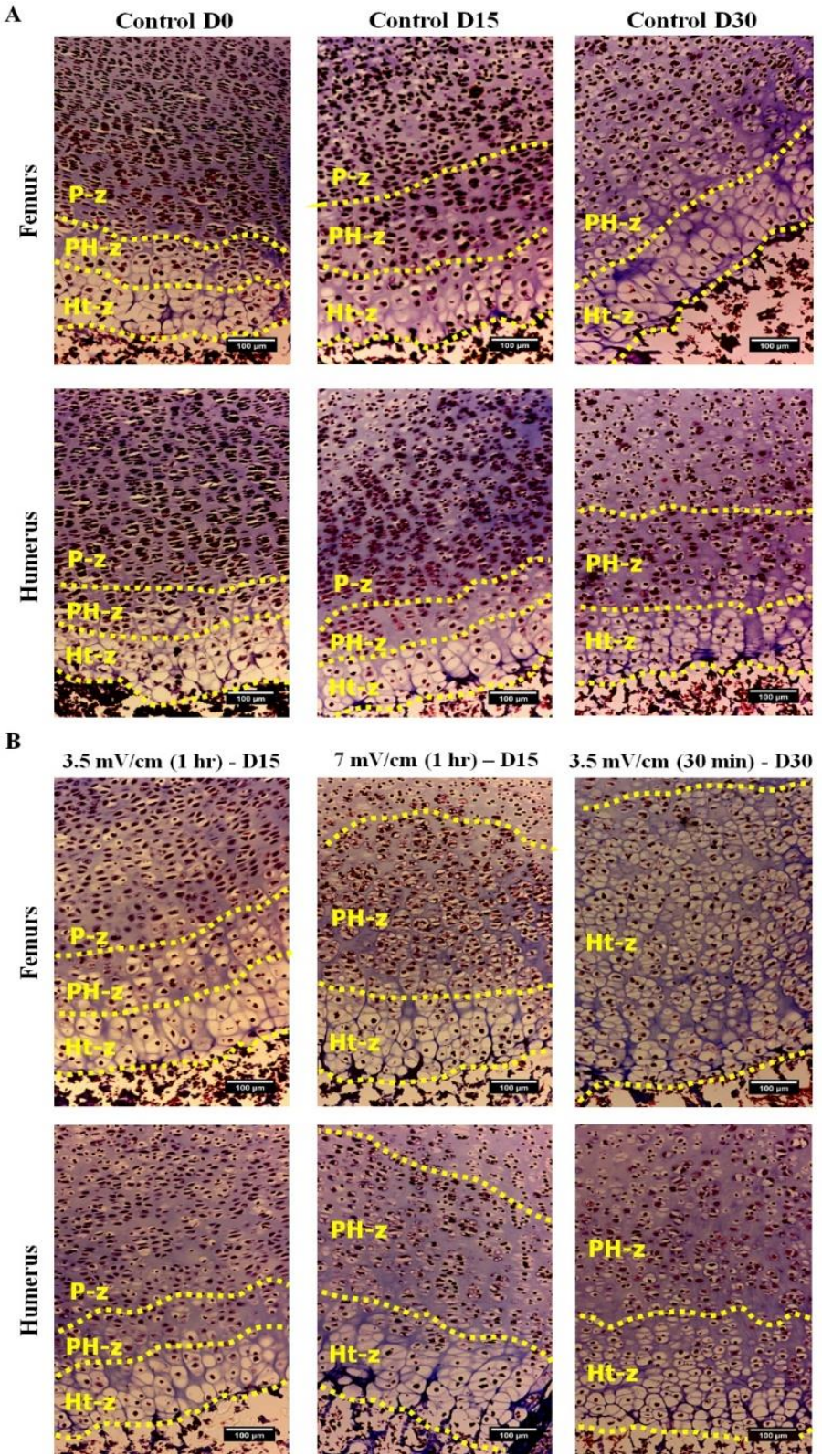

Figure 6-7. Histologies of growth plates at different times of culture.

A) Growth plates of control cultures. B) Growth plates of femurs and humerus after stimulation with EFs. Yellow dotted lines separate the growth plate from calcified bone. Scale bar $=100 \mu \mathrm{m}$. 
This behavior was evidenced in Figure 6-7A, where it is possible to observe that columnar chondrocytes are randomly distributed within the tissue, except in those epiphyseal plates stimulated with $3.5 \mathrm{mV} / \mathrm{cm}$ for 30 min where the P-z remained stable (See Figure 6-7B). The PH-z and $\mathrm{Ht}-\mathrm{Z}$ were visible in the epiphyseal plate in both controls and stimulated cultures; however, each zone had a different behavior. The PH-z increased in thickness as the EFs were higher, while the Ht-z was higher with EFs of $3.5 \mathrm{mV} / \mathrm{cm}$ in comparison with those stimulated with EFs of $7 \mathrm{mV} / \mathrm{cm}$ (See Figure 6-6B and Figure 6-7B). The thickness of PH$\mathrm{z}$ and $\mathrm{Ht}-\mathrm{z}$ also changed according to the period of culture. For instance, the PH-z at D15 was higher in explants stimulated with $7 \mathrm{mV} / \mathrm{cm}$ in comparison with those stimulated with $3.5 \mathrm{mV} / \mathrm{cm}$. However, a reverse behavior was observed at D30 in which the PH-z decreased in presence of EFs of $7 \mathrm{mV} / \mathrm{cm}$, while this zone experienced an increase with EFs of $3.5 \mathrm{mV} / \mathrm{cm}$. Regarding the Ht-z, the thickness of this zone in explants stimulated with $3.5 \mathrm{mV} / \mathrm{cm}$ was higher at D15, in comparison with those stimulated with $7 \mathrm{mv} / \mathrm{cm}$. No significant changes were observed at D30 (Figure 6-6B). The growth plate width and the thickness of each zone in humerus explants are shown in Appendix $\mathbf{H}$.

\subsubsection{Columnar analysis of growth plate zones}

Cells not forming columns $\left(C_{i}\right)$ and the chondrocytes that stratified into columns $\left(C_{c}\right)$ in femur explants are shown in Figure 6-8. Results evidenced that the number of $C_{i}$ vary depending on the zone of the growth plate, due to the fact that chondrocyte density tended to be higher at P-z and lesser in Ht-z at D15. A similar behavior was observed in explants stimulated with $3.5 \mathrm{mV} / \mathrm{cm}$ for $1 \mathrm{~h}$ where there was the same proportion of $C_{i}$ in the growth plate zones. It was possible to observe that chondrocytes which were not forming columns were higher in $\mathrm{P}-\mathrm{z}$ and PH-z, while in Ht-z tended to be lesser (Figure 6-8A). Regarding the number of $C_{c}$, control cultures evidenced that there are more chondrocytes forming columns in $\mathrm{P}-\mathrm{z}$ than in $\mathrm{PH}-\mathrm{z}$ and Ht-z (Figure 6-8B). This behavior was similar in bones stimulated with $3.5 \mathrm{mV} / \mathrm{cm}$ during $1 \mathrm{~h}$. In bones exempt of P-z, the number of $C_{c}$ varied according to the EF applied; for instance, chondrocytes forming columns tended 
to increase in PH-z and Ht-z with EFs of $7 \mathrm{mV} / \mathrm{cm}$ applied either for 30 min or $1 \mathrm{~h}$. The quantification of $C_{i}$ and $C_{c}$ of femurs explants are shown in Appendix I.

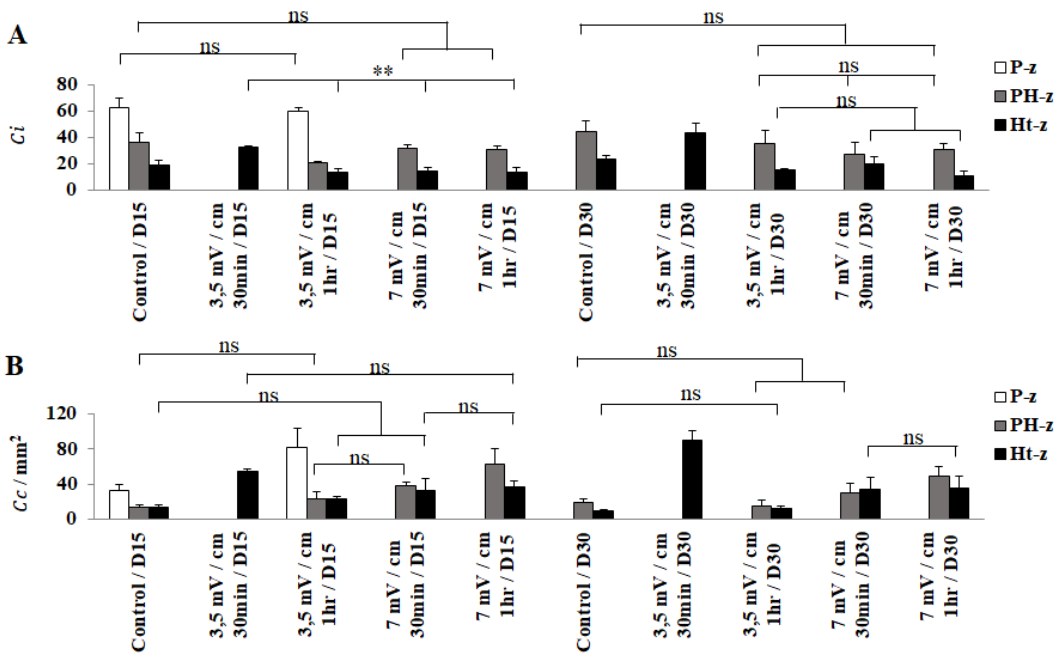

Figure 6-8. Measurement of $C_{i}$ and $C_{c}$ in growth plate zones of femur explants.

A) Graphic bars of $C_{i}$ within growth plate zones of femurs. B) Graphic bars of $C_{c}$ within growth plate zones of femurs. Statistically significant differences were found between controls and stimulated explants after a stimulation with EFs of 3.5 and $7.5 \mathrm{mV} / \mathrm{cm}\left(p<0,05^{* *}\right)$, except those marked with "ns" (not significant).

The parameters that indicate the number of columns $\left(C_{D}\right)$ and their inclination angles $\alpha$ within the growth plate zones of femur explants are shown in Figure 6-9. Some differences can be found between treatments in terms of the number of $C_{D}$ per zones. For example, explants stimulated for 15 and 30 days had more $C_{D}$ in the PH-z and Ht-z compared with the $C_{D}$ in the P-z (Figure 6-9A). The parameter of $C_{D}$ has a directly proportional behavior with $C_{c}$ according to the observed between Figure 6-8B and Figure 6-9A. It means that columns in each zone depend directly on the number of stacked chondrocytes. Even though column of cells were observed in the different zones of the 
growth plate during culture (Figure 6-7), not all columns were perpendicularly aligned to the axis of bone growth. This behavior can be corroborated in Figure 6-9B, where it was possible to observe that at D30 the variation coefficient of the angle decreased for stimulated cultures. It means that columns were formed but they experienced a random growth. In terms of the inclination angle of columns $(\alpha)$, it was observed that variation of the angle tended to be similar between controls and stimulated explants at D15. Nevertheless, variation decreased for stimulated cultures at D30 $\alpha$, which means that columns experienced a random growth (Figure 6-9B). This behavior was much more evident in humerus explants than in femurs (See Appendix J).

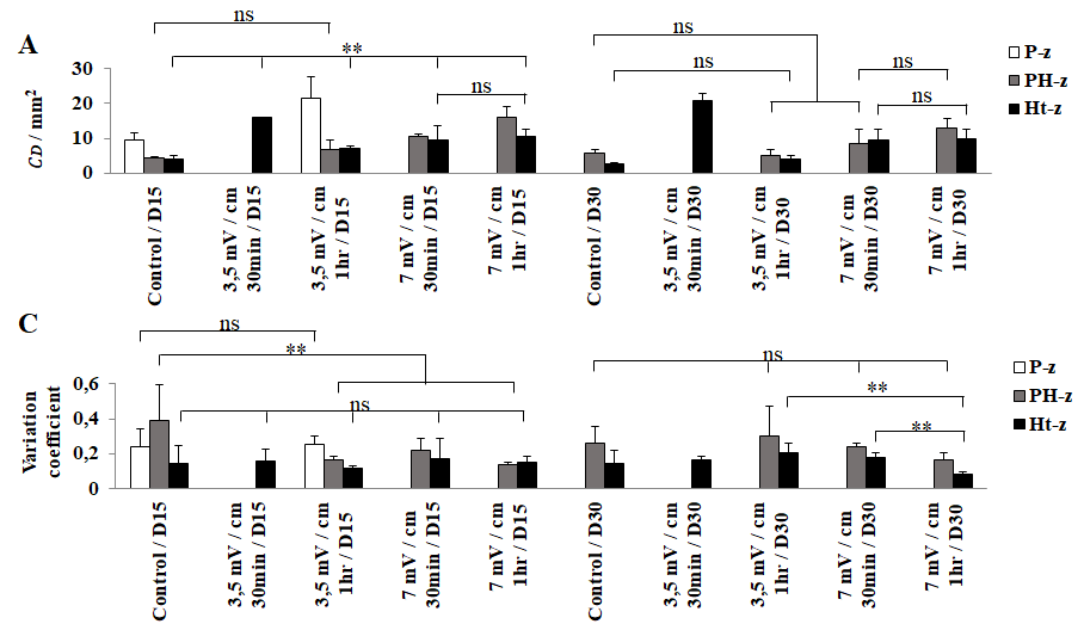

Figure 6-9. Measurement of $C_{D}$ and inclination angle $\alpha$ of columns in growth plate zones of femur explants.

A) Graphic bars of $C_{D}$ within growth plate zones of femurs. $B$ ) Graphic bars of $\alpha$ within growth plate zones of femurs. Statistically significant differences were found between controls and stimulated explants after a stimulation with EFs of 3.5 and $7.5 \mathrm{mV} / \mathrm{cm}(p<$ $0,05^{* *}$ ), except those marked with "ns" (not significant).

The Masson's trichrome staining not only allowed to observe chondrocyte morphology in the growth plate zones, but also allowed to evidence a differentiation process in the chondroepiphysis of stimulated bones at D30 (Figure 6-10). A group of hypertrophic chondrocytes 
forming a circle was stained in the center of the epiphysis of bones stimulated with EFs of 3.5 and $7 \mathrm{mV} / \mathrm{cm}$ during $1 \mathrm{~h}$ (Figure 6-10Figure 6-10B and Figure 6-10C). No evidence of this differentiation process was observed in control cultures (Figure 6-10A).

A

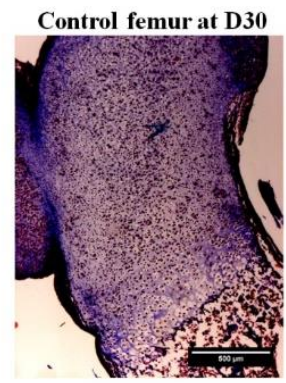

B

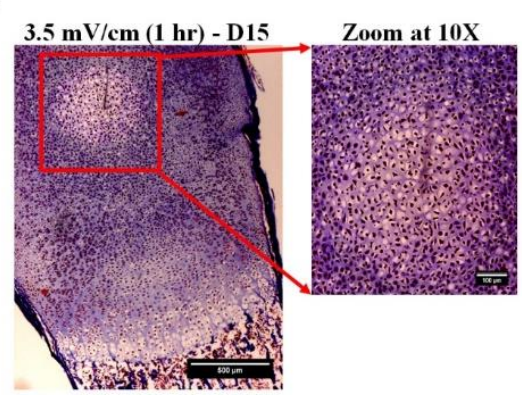

C

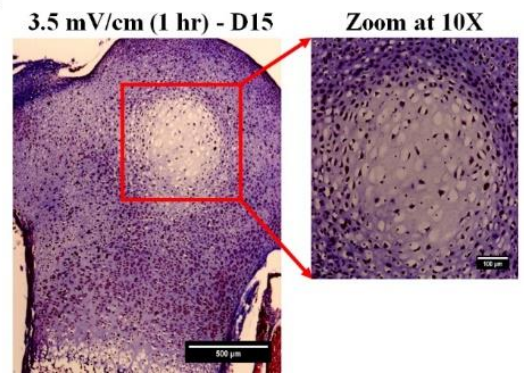

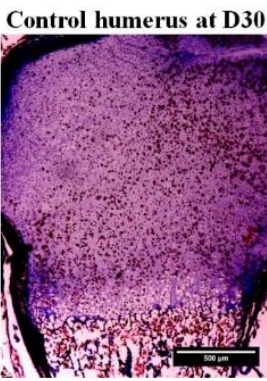
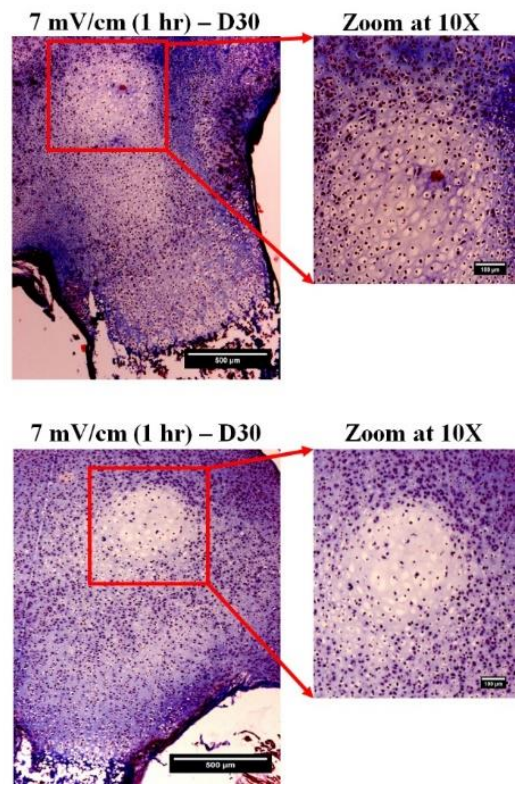

Figure 6-10. Representative scheme of histologies showing a hypertrophic cell condensation in the center of the chondroepiphysis of femurs and humerus.

A) Histologies of femur and humerus controls at D30 of culture. B) Histologies of stimulated femurs at D15 and D30. C) Histologies of stimulated humerus at D15 and D30. Scale bar for bone histologies = $500 \mu \mathrm{m}$. Scale bar for zoom histologies at 10X $=100 \mu \mathrm{m}$. 


\subsection{Discussion}

Biophysical stimuli belong to the wide range of environmental stimuli to which cartilage cells are exposed. In fact, it has been demonstrated that chondrocytes are able to sense and trigger specific responses to different types of stimulation such as compressive loads, tension, hydrostatic pressure, shear stress and electrostimulation. Regarding chondrocytes in the growth plate, the main biophysical stimulus sensed by these cells is mechanical loading. Experimental studies have shown that mechanical compression has effects over chondrocytes in terms of dynamics (proliferation, hypertrophy and apoptosis) [198], [201], [294], cell morphology [203], [305], protein synthesis of chondrocytes (collagen type II, $\mathrm{X}$ and aggrecan) and enzyme expressions (MMP 13 and ADAMTS-4/5) [204], [205]. Accordingly, it is possible to argue that the absence of mechanical loading corresponds to alterations in growth plate function, leading to abnormal shape and length of one or various skeletal elements. Studies in vivo and in vitro have also demonstrated that chondrocytes are also affected by electrical and electromagnetic stimuli, resulting in changes in cell dynamics, such as migration, differentiation, morphology, proliferation and gene expression [147], [151], [154]-[156], [164], [178], [299]. To approach such issue, a histomorphometric approach was used to evaluate the effect of capacitively coupled EFs on bone explants cultured in vitro.

Results showed that the columnar zone on the stimulated bones were usually wider, and that cell columns were longer with many mature cells (hypertrophic). This result corroborates the findings of [180], in which an extra growth and longitudinal arrangement of immature cartilage cells in the Ht-z was observed. A similar work carried out by [182] showed that a direct stimulation on the bone increased the Ht-z of the growth plates; however, an abnormal growth of the epiphyseal plate was observed due to an incorrect application of the EFs according to the author. In this context, the correct intensity of the EFs applied are a relevant factor to consider, because high voltages can reduce bone growth and maintain cartilage in a quiescent state [183]. Taking this into account, the EFs, the stimulation time and the period of culture 
were well-stablished parameters in order to obtain a better understanding about the effect of electrical stimulation over the growth plate.

Considering parameters about the EFs and frequencies established in literature [155], [167], [178], [181], [299], it was possible to obtain relevant results about the morphophysiology of chondrocytes within the epiphyseal plates. Although these results are important to understand how cells react to an external stimulus, the mechanism by which cells sense an external signal and then, translate it into a molecular response remains unknown. It has been evidenced that ion channels might be associated to electrical signal transduction. Even though a plethora of ion channels has been identified in chondrocytes membranes, calcium channels are the main type of ion channels associated to transduction of biophysical stimulations. Electrical signals applied either in indirect or direct contact with the cells, exert their effect on the cell membrane by activating the VGCC leading to increase in the intracellular $\mathrm{Ca}^{2}+$ levels [260], [306], [307]. The elevated influx of $\mathrm{Ca}^{2}+$ activates the cytoskeletal calmodulin, an intracellular protein that regulates the signal transduction of calcium within the cell, allowing the production of certain transcription factors such as the SOX-9 molecule. The activation of SOX-9 triggers an intracellular production of the main molecules of the articular cartilage such as collagen type II and aggrecan [260], [297], [298]. Although this signaling pathway was not implicated in this study, this aspect should be the focus of future works to clarify how EFs act over the chondrocytes from an electro-biological point of view. Another signaling pathway activated by EFs is the Wnt $/ \beta$-catenin. This protein, also recognized as a morphogen, is involved in several developmental processes including body axis patterning, as well as cell differentiation, proliferation and migration [308], [309]. In fact, a study demonstrated an increase of $\beta$-catenin in the chondrocytes nucleus after an stimulation with EFs [154]. These results allow to conclude that electrical stimulation is a promising tool, as this biophysical stimulus may potentially alter biochemical, biophysical and electrochemical properties of chondrocytes [161], [162], [164], [167], [260], [298]. 
The histomorphometric analysis was carried out in bone explants isolated after birth. At this stage the internal structures of bones, such as the primary ossification center and the epiphysis are already formed. Considering that bones have a different behavior in vivo, where the process of bone formation continues, the results obtained cannot be compared with growth plate of bones that have grown in vivo for 30 days. A bone presents a well-formed structure at D30, highlighting the primary and secondary ossification centers and growth plates. Although the aim of this study was not to reproduce the endochondral ossification process, the in vitro experiments allowed to observe the hypertrophic evolution of chondrocytes in response of a biophysical stimuli. Thus, it is noteworthy to mention that EFs have influence in the differentiation process not only over the growth plates, but also over the center of the epiphysis where chondrocytes increased their cellular volume (Figure 6-10). This finding is relevant since EFs demonstrated to have influence over the pre-hypertrophy. This is an important event that plays a pivotal role, as it is responsible of the $70 \%$ of total longitudinal bone growth, in the initiation of the matrix mineralization, and in the promotion of tissue vascularization [303]. Although the mechanisms by which capacitively coupled EFs stimulate chondrocytes differentiation are not yet known, the observed effects in the immunohistochemistry staining evidenced that chondrocytes experience a pre-hypertrophic process at the location of the secondary ossification center (Figure 6-10B and Figure 6-10C).

Based on the above, understanding the influence of electrical stimulus in the biology of the growth plate not only provides useful information regarding their role in epiphyseal plate physiology in vivo, but also supplies new tools for tissue engineering and regenerative medicine focused on the development of new therapeutic approaches for the treatment of injuries in the growth plate.

\subsection{Concluding Remarks}

The histomorphometric approach used to evaluate the effect of capacitive coupled EFs on bone explants revealed that growth plates morphology varies combining three main factors: 1) the EFs intensities, 
2) stimulation time per day and 3) period of culture. Thus, electrical stimulation has the ability to precisely control physical and biological properties of the growth plate by modifying either the thickness of the tissue or altering the cell response. Overall, the combination of biophysical techniques and in vitro culture models will not only allow the creation of novel tools to study the behavior of tissues, but also they can be used to create new therapies focused on the treatment of chronic pathologies of hyaline cartilage. The methodologies used to stimulate the regeneration of cartilage with EFs are increasingly approaching the development of technologies that meet the requirements of successful cartilage healing. In addition to that, electrical stimulation as a biophysical stimulus used in cartilage tissue engineering may improve clinical settings through the development of devices that stimulate in vivo tissues in regenerative therapies. 



\section{CHAPTER 7}

\section{Electrical stimulation enhances chondrogenic differentiation of mesenchymal stem cells cultured in hyaluronic acid - gelatin hydrogels}





\subsection{Summary ${ }^{6}$}

Hyaline cartilage degenerative pathologies induce morphological and biomechanical changes that lead to cartilage tissue damage. In pursuit of a treatment to restore cartilage, injectable hydrogels have been used as a three-dimensional network to promote chondrogenic differentiation in situ in injured zones. However, factors such as culture times, cell viability and cellular retention within the matrix are not wellestablished. In addition, a critical point in the use of hydrogels is that they alone cannot maintain a prolonged chondrogenesis process. Electrical stimulation has been proposed as a therapeutic option to improve tissue engineering techniques focused on cartilage repair, since there is evidence that electric fields induce the differentiation of mesenchymal stem cells towards the chondrocytic lineage. Our previous research has demonstrated that hydrogel mixtures of hyaluronic acid and gelatin are able to induce the chondrogenic phenotype of mesenchymal stem cells in in vitro cultures without the need of specific growth factors. Accordingly, the aim of this study was to electrically stimulate mesenchymal stem cells encapsulated in the optimal hydrogel mixture to enhance the chondrogenic differentiation previously observed. Results evidenced that an electric field of 10 $\mathrm{mV} / \mathrm{cm}$ at $60 \mathrm{kHz}$, applied for $30 \mathrm{~min}$ four times per day during 21 days, promotes cell proliferation and chondrogenic differentiation. The proliferation rate increased in stimulated hydrogels after 14 days of stimulation, while the expressions of SOX-9 and aggrecan were higher in stimulated hydrogels after 21 days of stimulation. These findings allow to conclude that electric stimulation is a very promising tool to enhance articular cartilage tissue engineering outcomes in therapies that combine hydrogels and mesenchymal stem cells.

\footnotetext{
${ }^{6}$ The results presented in this chapter are in preparation to be published as a scientific article: J.J. Vaca-González, S. Clara-Trujillo, M. Guillot-Ferriols, J. RódenasRochina, M.J. Sanchis Sánchez, J.L. Gómez Ribelles, D.A. Garzón-Alvarado and G. Gallego Ferrer, "Coupled Capacitive Electric Fields Over Hyaluronic Acid - Gelatin Hydrogels Enhance Chondrogenic Differentiation". (In preparation).
} 


\subsection{Introduction}

Articular cartilage acts as a low-friction and wear-resistant surface in the load-bearing synovial joints [310]. Given its damping function, the cartilaginous tissue requires a specialized molecular structure to resist mechanical loads. Accordingly, the chondrocyte, the unique cellular type within cartilage, synthetize and maintain the molecular structure of the extracellular matrix (ECM) of the cartilage, such as collagens and proteoglycans (PGs) [1], [5]. These molecules provide greater stiffness, elasticity and resistance to osmotic and cyclic compression to the cartilaginous tissue [1], [311], [312]. The cartilage has limited selfhealing properties when is affected by traumatic injuries because it is an aneural tissue with low chondrocyte density [46], [280]. In this context, novel therapeutic methods have been implemented to recover the cartilage such as autologous mesenchymal stem cells (MSCs) implantation and intra-articular injections of MSCs [1], [46], [278], [280]-[282]. Considering that MSCs are multipotent cells capable to differentiate into several cells types such as cardiac muscle cells, neural cells, adipocytes, osteoblasts and chondrocytes [313], the aforementioned techniques are based on obtaining patient MSCs, expand them through in vitro cultures, and re-implanted them into the injured tissue [282], [314]. The autologous implantation has had midterm effects in cartilage repair, and the treated zone are primarily filled with fibrocartilage tissue in several cases [315]. Regarding the intraarticular injections, it has been observed that MSCs encapsulated in hyaluronic acid (HA) solutions and injected in osteoarthritic knees have improved the treatment outcomes and the rate of cartilage regeneration [316]. MSCs have also shown their chondrogenic capacity to treat cartilage in the presence of growth factors and three-dimensional (3D) environments [317]. In fact, 3D structures provide ideal environment to the MSCs to proliferate, migrate and differentiate into functional tissuespecific cells. Furthermore, 3D constructs enhance the diffusion of nutrients and bring a mechanical support for the cells in the first stages of regeneration [318]. Implement a biomimetic material with molecular properties similar to those of articular cartilage is challenge nowadays; in fact, different 3D constructs have been implemented to assess the cellular dynamics of MSCs and promote chondrogenesis such as 
alginate beads [319], [320], agarose hydrogels [321]-[323], collagen fiber [65], [66], micro-particles made of different polymers [63], [324], [325], and hyaluronic acid hydrogels [89], [326]-[328].

Previous studies have indicated that injectable HA - gelatin hydrogels (HA-GEL) maintain chondrocytes morphology and enhance gene expression of collagen type II and aggrecan [93], [329]. It has been also demonstrated that HA is biocompatible and biodegradable substrata that enhances chondrogenesis process [310]. For instance, Pfeifer et al. evidenced that human MSCs embedded in HA-GEL hydrogels synthetized cartilage molecules such as collagen type II, GAGs, TGFbeta and melanoma inhibitory activity protein (MIA) [92]. Similarly, some studies have shown that chondrogenesis is improved in the absence of growth factors such as TGF- $\beta 3$, as was the case with the mixture of 70\% HA - 30\% GEL reported by Moulisová et al., [85], the collagen - HA scaffolds developed by Murphy et al., [330], and the decellularized cartilage matrices investigated by Burnsed et al., [331]. Other feature to highlight from the HA-GEL hydrogels is the ability to precisely control physiochemical and mechanical properties such as shear modulus, stiffness, water permeability and swelling and crosslinking degree [85], [332]-[334].

Despite of this, it was evidenced that the use of 3D culture environments alone cannot maintain a prolonged chondrogenesis [318]. For this reason, cartilage tissue engineering has established technologies such as the application of biophysical stimuli in order to enhance flow of nutrients, cell attachment, molecular synthesis production and a prolonged chondrogenesis [335]. In this context, it has been reported that MSCs cultured either in monolayer or 3D constructs respond to a biophysical stimulus such as electric fields (EFs). For instance, in vitro studies have shown that EFs applied over MSCs cultured in monolayer change cell morphology and cell alignment [336], [337], improve cell migration [338], [339], and increase cell proliferation [340]. Regarding 3D cultures, it has evidenced that micromass MSCs experienced an increase in collagen type II, aggrecan and SOX-9 after an stimulation with EFs [341]-[343]. 
Taking the above mentioned, we hypothesize that electric field stimulation may improve the positive results observed with MSCs in HA-GEL hydrogels. Since, to the best of our knowledge, there are not reported studies addressing such issue, the aim of this study was to stimulate mesenchymal stem cells encapsulated into injectable hydrogels in order to enhance the chondrogenic differentiation. To accomplish this, a capacitively coupled system was designed and implemented to generate and homogeneously distribute EFs of 10 $\mathrm{mV} / \mathrm{cm}$ at $60 \mathrm{kHz}$ (sine wave-form). The hydrogels were stimulated using a stimulation scheme that consisted in applying EFs for $30 \mathrm{~min}$ four times per day during 21 days of culture. The mechanical properties of the hydrogels were measured by rheology, while the dielectric properties of the hybrid were measured by dielectric and impedance spectrometry. Cell proliferation was assessed by colorimetry, while gene expressions of collagen type I and II, SOX-9 and aggrecan were measured through immunofluorescence and RT-qPCR analyses. Results evidenced that electrical stimulation increased cell proliferation and enhanced the synthesis of chondrogenic markers such as SOX-9 and aggrecan. In this study, the combination of 3D structures and EFs not only create a novel methodology to stimulate chondrogenesis in the absence of growth factors, but also may be used improve the therapeutic approaches focused on the treatment of chronic pathologies of articular cartilage. The methodologies used to stimulate the regeneration of cartilage are increasingly approaching the development of technologies that meet the requirements of successful cartilage healing and make the concept of cartilage tissue engineering extrapolated to a purely clinical setting.

\subsection{Materials and methods}

\subsection{1}

\section{Materials}

For the hydrogel synthesis the following reagents were employed: Hyaluronic acid sodium salt from Streptococcus equi, gelatin strength 300 type A, 2-( $N$-morpholino) ethanesulfonic acid (MES), tyramine hydrochloride (HCl-Tyramine), N-Hydroxysuccinimide (NHS), dialysis tubing of 12.400 molecular weight cutoff (MWCO), sodium 
chloride $(\mathrm{NaCl})$, hydrochloric acid $(\mathrm{HCL})$, sodium hydroxide $(\mathrm{NaOH})$, hydrogen peroxide solution $\left(\mathrm{H}_{2} \mathrm{O}_{2}\right)$, peroxidase from horseradish (HRP). All reagents were purchased from Sigma-Aldrich. Dialysis Tubing of 3.500 MWCO was acquired from Spectrum labs and carbodiimide hydrochloride (EDC) was obtained from Iris Biotech GMBH.

For the biological experiments DMEM (high glucose, GlutaMAX Supplement), Fetal Bovine Serum (FBS), phosphate buffered saline (PBS), antibiotics (penicillin/streptomycin) and trypan blue stain were obtained from Gibco. All the other reagents used in experiments were CellTiter 96 AQueous One Solution Cell Proliferation Assay (MTS) from Promega, recombinant human fibroblast growth factor basis (FGF-2) from Eurobio, saccharose from Panreac Química, Tween-20, DAPI dilactate, Triton X-100 and bovine serum albumin (BSA) from Sigma-Aldrich, QIAzol lysis reagent from Qiagen, chloroform from Fluka Analytical, absolute ethanol for molecular biology (100\%) from Fisher Bioreagents, isopropanol from Scharlab, OCT from Tissue-Tek, aggrecan primary antibody (BC-3) from Novus Biologicals, anti-SOX9 primary antibody from Abcam, secondaries antibodies for aggrecan (Alexa Fluor 555 goat anti-mouse), SOX-9 (Alexa Fluor 488 goat antirabbit), and phalloidin (Alexa flour 488) from Invitrogen, Maxima First Strand cDNA Synthesis Kit for RT-qPCR, with dsDNase and PowerUp ${ }^{\mathrm{TM}} \mathrm{SYBR}^{\mathrm{TM}}$ Green Master Mix from ThermoFisher Scientific.

The medium used to dissolve the polymers was the Calcium-free Krebs Ringer buffer (CF-KRB). This buffer was prepared in miliQ water with $115 \mathrm{mM} \mathrm{NaCl}, 5 \mathrm{mM}$ potassium chloride $(\mathrm{KCl}), 1 \mathrm{mM}$ potassium dihydrogen phosphate $\left(\mathrm{KH}_{2} \mathrm{PO}_{4}\right)$, and $25 \mathrm{mM}$ 4-(2-hydroxyethyl) piperazine-1-ethanesulphonic acid (HEPES). Other buffer used in this study was the Tris-Buffered Saline (TBS 10X) with $\mathrm{pH}$ of 7.6. This buffer was prepared with Tris $(24 \mathrm{gr})$ and $\mathrm{NaCl}(24 \mathrm{gr})$ in $1 \mathrm{~L}$ of distilled water. Reagent for buffers preparation were obtained from ThermoFisher Scientific. 


\subsubsection{Synthesis of HA-GEL hydrogels}

Considering the protocols described by [85], [344] and [345], the hydrogel mixture was obtained by enzymatically cross-linking tyramine conjugates of hyaluronic acid (HA-tyr) and gelatin (GEL-tyr), previously synthesized.

HA-tyr was obtained after two chemical modifications. First, pristine HA molecular weight was reduced from 1.2 MDa to $350 \mathrm{kDa}$ through acid degradation of a solution of $1 \% \mathrm{w} / \mathrm{v} \mathrm{HA}$ in deionized water. Then, the solution was adjusted at $\mathrm{pH} 0.5$ with $\mathrm{HCl}(20 \mathrm{M})$ and stirred at 200 rpm for $24 \mathrm{~h}$ at $37{ }^{\circ} \mathrm{C}$ for HA degradation. Then, $\mathrm{pH}$ was adjusted at 7 by adding a $5 \mathrm{M}$ solution of $\mathrm{NaOH}$. The obtained product was dialyzed for 3 days using a dialysis tubing of $3.500 \mathrm{MWCO}$, against deionized water that was changed three times per day. Finally, HA of low molecular weight was lyophilized in a LyoQuest freeze dryer (Telstar Life Science Solutions, Japan). In order to verify the molecular weight of the HA obtained, a measurement by Gel Permeation Chromatography (GPC), using a Waters Breeze system (Waters corporation, MA, USA), was performed according to the protocol described by [346]. Afterwards, the low molecular weight HA was grafted with tyramine. HA solution $(0.5 \%$ in deionized water) was dissolved in presence of $\mathrm{NaCl}(150 \mathrm{mM})$, MES $(0.276 \mathrm{mM})$ and $\mathrm{NaOH}$ $(5 \mathrm{M})$ at $\mathrm{pH} 5.75$. To guaranty a correct dissolution of the HA, the mixture was stirred at $500 \mathrm{rpm}$ for $2 \mathrm{~h}$ at room temperature. Then, $\mathrm{HCl}-$ Tyramine (2:1 Tyr/COOH molar ratio) was added and stirred during 20 min at room temperature, and the $\mathrm{pH}$ was adjusted to 5.75. Then, EDC (1:1 EDC/COOH molar ratio) and NHS (1:10 NHS/EDC molar ratio) were added and stirred for $24 \mathrm{~h}$ at $37^{\circ} \mathrm{C}$. After reaction, the dissolution was dialyzed in a tube of $3.500 \mathrm{MWCO}$ for 2 days against $\mathrm{NaCl}(150$ $\mathrm{mM}$ ) for $24 \mathrm{~h}$ and against deionized water for another $24 \mathrm{~h}$, with three changes of dialysis solution every day. Finally, the low molecular weight HA tyramine (HA-Tyr) grafted was lyophilized.

Gelatin solution ( $2 \%$ w/v in deionized water) was dissolved with MES $(50 \mathrm{mM})$ for $30 \mathrm{~min}$ at $60{ }^{\circ} \mathrm{C}$. Then, $\mathrm{HCl}$-Tyramine $(2: 1 \mathrm{Tyr} / \mathrm{COOH}$ molar ratio) was added and stirred during $20 \mathrm{~min}$ at room temperature. 
Then, the $\mathrm{pH}$ of the dissolution was adjusted at 6 and NHS was added and stirred at room temperature for $30 \mathrm{~min}$. Next, ECD (2:1 $\mathrm{EDC} / \mathrm{COOH}$ molar ratio) was added and the mixture was stirred for $24 \mathrm{~h}$ at $37^{\circ} \mathrm{C}$. Once the reaction time ended, the dissolution was dialyzed for 2 days using a dialysis tubing of 12.400 MWCO. The dialysis was done against deionized water, which was changed three times per day. Finally, the tyramine conjugate of gelatin (GEL-Tyr) was lyophilized for further use.

\subsubsection{Tyramine substitution degree in HA and GEL}

The amount of phenol groups grafted in the HA and GEL were measured by spectrophotometry. First, $0.1 \%$ w/w solutions of HA-Tyr and GEL-Tyr in deionized water were prepared. Absorbance of this solutions was measured at $275 \mathrm{~nm}$ with a Cary $60 \mathrm{UV}$-Vis spectrophotometer (Agilent Technologies, Santa Clara, CA, USA). The substitution degree for HA-Tyr and GEL-Tyr was obtained by calculating the number of moles of tyramine per $\mathrm{mL}$ of dissolution. Calibration curve used included tyramine patterns at the following concentrations prepared in deionized water: $0.0125,0.025,0.05,0.1$ and $0.125 \mathrm{mg} / \mathrm{ml}$. Then, the number of moles per each carboxyl group $(\mathrm{COOH})$ of the HA and gelatin was obtained in order to calculate the percentage of tyramine molecules per each $\mathrm{COOH}$ group. Regarding the $\mathrm{COOH}$ of pristine gelatin, the data was provided by the supplier and it is $80 \mathrm{mmol}$ of $\mathrm{COOH}$ per $100 \mathrm{~g}$ of gelatin. Concerning the $\mathrm{COOH}$ of the HA, we used the molecular weight of each monomeric unit $\left(\mathrm{C}_{14} \mathrm{H}_{21} \mathrm{NO}_{11}\right)$ and the mg of $\mathrm{HA}$ used in the measurements to calculate the moles of $\mathrm{COOH}$ per mg of pristine $\mathrm{HA}$.

\subsubsection{Hydrogels preparation}

Hydrogels $2 \%(\mathrm{w} / \mathrm{v})$ in a proportion of $70 \%$ HA $(2 \% \mathrm{w} / \mathrm{v})$ and $30 \%$ gelatin $(2 \% \mathrm{w} / \mathrm{v})$ were prepared according to the protocol described by [85], [344]. HA was dissolved $5 \mathrm{~h}$ before at $4{ }^{\circ} \mathrm{C}$, while gelatin was dissolved for $30 \mathrm{~min}$ at $37^{\circ} \mathrm{C}$. A mixture containing $80 \%(\mathrm{v} / \mathrm{v})$ of $2 \mathrm{w} / \mathrm{v}$ $\%$ of HA-GEL and $10 \%(\mathrm{v} / \mathrm{v})$ of HRP $(12.5 \mathrm{U} / \mathrm{mL})$ was prepared. Then, the HA-GEL-HRP solution was cross-linked with $10 \%(\mathrm{v} / \mathrm{v})$ of $\mathrm{H}_{2} \mathrm{O}_{2}$ 
(20 mM). The hybrid was incubated at $37{ }^{\circ} \mathrm{C}$ and $5 \% \mathrm{CO}_{2}$ for $20 \mathrm{~min}$ to ensure hydrogel cross-linking.

\subsubsection{Rheological measurements of HA-GEL hydrogel}

Three different measurements were performed in order to mechanically characterize the HA-GEL hydrogel. First, an oscillatory time sweep for $20 \mathrm{~min}$ to register the gelation dynamics of the hydrogel was performed. The strain and frequency were selected at $1 \%$ and $1 \mathrm{~Hz}$, respectively. Second, a dynamic strain sweep to calculate the range of strain amplitudes was carried out. Amplitudes ranging between $0.01 \%$ and $15 \%$ and a frequency of $1 \mathrm{~Hz}$ to measure the dynamic shear modulus as a function of strain were used. Finally, a dynamic frequency sweep test to determine the dependence of the dynamic shear modulus and loss factor on the frequency was done. Frequencies between 0.1 and $10 \mathrm{~Hz}$ and a strain of $1 \%$ were selected. The hydrogel temperature was controlled and maintained by a Peltier device at $37^{\circ} \mathrm{C}$. The hydrogel dynamic was measured using a gap around $1100 \mu \mathrm{m}$ between the plates. During the experiment, the solvent loss was reduced using a solvent trap geometry of parallel plates (made of nonporous stainless steel, diameter $=20 \mathrm{~mm}$ ). Measurements were carried out by cross-linking HA ( $2 \mathrm{w} / \mathrm{v} \%)$ and gelatin ( $2 \mathrm{w} / \mathrm{v} \%)$ either in DMEM or CF-KRB with $1 \% \mathrm{P} / \mathrm{S}$ at $37{ }^{\circ} \mathrm{C}$ in order to determine which medium has similar mechanical properties to those of cartilage tissue. The protocol described for cross-linking process of hydrogels is showed in section 7.3.4. The rheological parameters were measured using a straincontrolled rheometer (Discovery HR-2, TA Instruments, New Castle, DE USA).

\subsubsection{Dielectric constants of HA-GEL hydrogels and culture media}

Dielectric experiments were performed to measure the conductivity and permittivity of the hydrogel mixture and the culture media. A volume of $350 \mu \mathrm{L}$ either of hydrogel mixture $(2 \% \mathrm{w} / \mathrm{v})$ or culture media was placed in a liquid parallel plate sample cell (BDS1308). The hydrogels were gelified in situ over the electrode adding HA, gelatin, enzyme 
(HRP) and peroxide $\left(\mathrm{H}_{2} \mathrm{O}_{2}\right)$, as previously described. The electrode gap was adjusted by silica spacers. The measurements were carried out a room temperature and a frequency window from $1.1 \times 10^{0}$ to $1 \times 10^{7} \mathrm{~Hz}$ using a Novocontrol BDS system comprising a frequency response analyzer (Solartron Schlumberger FRA 1260) and a broad-band dielectric converter with an active sample head. The measurement error was shown to be less than $\pm 3 \%$.

\subsubsection{Estimation of EFs}

A computational simulation was implemented to estimate the EF in the capacitively coupling system and within the hydrogel. The coupled capacitive system is composed of two parallel stainless-steel electrodes located at the top and bottom of a 48 culture well-plates (Chapter 5 Figure 5-2). The EF generated between the electrodes is perpendicular to the hydrogels and it is influenced the potential differential applied between the electrodes $(100 \mathrm{~V}$ at $60 \mathrm{kHz})$ and the dielectric constants of the substrates (Table 7-1).

Table 7-1: Dielectric properties and measurements used in the simulation.

\begin{tabular}{llc}
\hline Component & \multicolumn{1}{c}{ Parameter } & $\begin{array}{c}\text { Value used in } \\
\text { simulation }\end{array}$ \\
\hline \multirow{5}{*}{ Stainless-steel } & Electrode separation & $20[\mathrm{~mm}]$ \\
& Electrode radius & $50[\mathrm{~mm}]$ \\
& Relative permittivity & $5[\mathrm{~mm}]$ \\
& Electric conductivity & $1.73913[\mathrm{MS} / \mathrm{m}]$ \\
& Length and height & \\
\multirow{4}{*}{ Well plate } & Thickness & $1[\mathrm{~mm}]$ \\
& Relative permittivity & 3.5 \\
& Electric conductivity & $6.9[\mathrm{nS} / \mathrm{m}]$ \\
Air & Relative permittivity & 1 \\
& Electric conductivity & $0[\mathrm{~S} / \mathrm{m}]$ \\
\hline
\end{tabular}

A single well-plate from a 48 well-plate was modelled as an axisymmetric configuration and the electrodes were big enough at the 
top and bottom of the single well to ensure a homogeneous and isotropic distribution of the EF during the simulation. The computational simulation was implemented by a finite element analysis using a software of electromagnetic simulations (COMSOL Multiphysics, Comsol Inc. Los Angeles, USA).

\subsubsection{Mesenchymal stem cell isolation and cell culture}

4-months-old pig leg obtained from the Veterinary Medicine Faculty of Universitat de València was used in this study to extract the MSCs. Recommendations from the ethics committee were followed for this experimental procedure. First, the skin and all surrounded muscles were carefully detached until the femur was completely exposed. Then, the proximal epiphysis of the femur was removed, and the bone marrow was extracted from the cancellous portion of the femur. The bone marrow was resuspended in $40 \mathrm{~mL}$ of DMEM, homogenized with a syringe and centrifugated at $1500 \mathrm{rpm}$ for $5 \mathrm{~min}$. This process was performed until the fat phase from the cell suspension was completely removed. Then, cell suspension was transferred into $10 \mathrm{~mL}$ of DMEM, homogenized and filtered using a membrane filter of $40 \mu \mathrm{m}$. Cell yield and viability were determined by counting in hemocytometer with trypan blue and $6 \%$ of acetic acid, respectively. 25 million of cells were cultured in T-75 culture flasks in DMEM supplemented with 10\% FBS and $1 \%$ antibiotics (penicillin/streptomycin). Cell cultures remained in atmospheric conditions of $37{ }^{\circ} \mathrm{C}$ and $5 \% \mathrm{CO}_{2}$, and culture media was changed every two days until a confluence of $80 \%$ was reached. Before cell encapsulation into the hydrogels, the MSCs were expanded until passage 3-4 in DMEM supplemented with 10\% FBS, $1 \%$ of antibiotics, and FGF-2 (50 ng/mL).

Hydrogels in a proportion of $70 \% \mathrm{HA}(2 \% \mathrm{w} / \mathrm{v})$ and $30 \%$ gelatin ( $2 \%$ $\mathrm{w} / \mathrm{v}$ ) were prepared as previously described in section 7.3.4. The lyophilized powders of their tyramine conjugates were dissolved in CFKRB for cell cultures. Once HA, gelatin and HRP were mixed, the solution was filtered through a $0.22 \mu \mathrm{m}$ syringe filter for sterilization. A required amount of MSCs $\left(1 \times 10^{6}\right.$ cells $\left./ \mathrm{mL}\right)$ from passage 4 to 5 were added to the HA-GEL-HRP solution [85]. Then, a drop of $45 \mu \mathrm{L}$ 
of the HA-GEL cell suspension was cross-linked with $5 \mu \mathrm{L}$ of $20 \mathrm{mM}$ $\mathrm{H}_{2} \mathrm{O}_{2}$ on each well of a 48 culture well-plate and left in an incubator at $37^{\circ} \mathrm{C}$ and $5 \% \mathrm{CO}_{2}$ for 20 min to ensure hydrogel cross-linking. Finally, a growth medium (GM) containing DMEM supplemented with $10 \%$ FBS and $1 \%$ of antibiotics was added. Cell culture were followed for 21 days, and cell culture medium was changed every 2 days.

\subsubsection{Electrical stimulation assay}

Electrical stimulation was performed using a coupled system that deliver homogeneous EFs [178]. The electrodes were placed in teflon supports to eliminate any contact with the incubator surface. The positive and negative terminals of the electrodes were connected to an electronic circuit (oscillator) that generated the voltage and frequency required to create the EF. The oscillator was energized with a dual source (Lendher - HY3003D-3, Shenzhen, China) and signal verification before and after electrical stimulation was monitored using an oscilloscope (Keysight - DSO1052B, Santa Rosa, CA, USA). Stimulated cultures were placed between electrodes, while control cultures were incubated in the same way, except that electrodes were not connected to the oscillator. Hydrogels were exposed to an EF of 10 $\mathrm{mV} / \mathrm{cm}$ at $60 \mathrm{kHz}$ sine wave-form during 21 days of culture. According to a previous report the EF was delivered at first day of culture using an exposure time of $30 \mathrm{~min}$ delivered four times per day according to following the scheme: $30 \mathrm{~min}$ of stimulation corresponds to $5.5 \mathrm{~h}$ without stimulation [178].

\subsubsection{Morphology and cell proliferation assay}

Morphology was assessed by microscopic examination after DAPI/actin fluorescent staining of the MSCs encapsulated in the hydrogels. First, all samples were washed with PBS, fixed in $4 \%$ of formaldehyde for $20 \mathrm{~min}$ at room temperature, and washed twice with PBS to remove fixing solution. Then, hydrogels were submerged overnight into $30 \% \mathrm{w} / \mathrm{v}$ saccharose in miliQ water, embedded in OCT and stored at $-80^{\circ} \mathrm{C}$. For DAPI/actin staining, the cryopreserved hydrogels were segmented at $30 \mu \mathrm{m}$ in a cryostat (Leica CM $1860 \mathrm{UV}$ ), 
placed over SuperFrost slides and stored overnight at room temperature. The slices were washed twice with PBS 1X. Then, the samples were washed with Triton $100 \mathrm{X}$ at $0.1 \% \mathrm{v} / \mathrm{v}$ in PBS $1 \mathrm{X}$ for $10 \mathrm{~min}$. Thereafter, the blocking solution was removed and two washes with PBS $1 \mathrm{X}$ were done. After, DAPI at 1:500 and phalloidin at 1:100 diluted in PBS 1X were added to the samples and incubated during $1 \mathrm{~h}$ at room temperature. Finally, the samples were washed twice with PBS $1 \mathrm{X}$ and stored at $4{ }^{\circ} \mathrm{C}$ for further examination under the fluorescence microscope (Nikon Eclipse 80i, Melville, NY USA).

Proliferation was assayed on days 2, 7, 14 and 21 using MTS assay. First, culture media was removed and the hydrogels (five replicates) were washed with PBS. Then, samples were transferred into new 48 culture well-plate, and a solution containing culture media without phenol red and MTS reagent (ratio 5:1) was added. Cell-cultured samples were incubated at $37^{\circ} \mathrm{C}$ and $5 \% \mathrm{CO}_{2}$ for $2 \mathrm{~h}$ in dark. Thereafter, the absorbance of $100 \mu \mathrm{L}$ of supernatant was measured at 490 and 690 $\mathrm{nm}$ with a VICTOR ${ }^{3}$ multilabel plate reader (Perkin Elmer, Waltham, MA). Absorbance at $490 \mathrm{~nm}$ is proportional to the number of viable cells in each sample, whereas the absorbance at $690 \mathrm{~nm}$ is used to subtract the potential background signal of small pieces of hydrogels inside each well. Additionally, cell morphology was assessed using phase contrast images on days 2, 7, 14 and 21 using an inverted microscope (Nikon Eclipse TS100, Melville, NY USA).

\subsubsection{Assessment of chondrogenic markers}

Hydrogels at days 14 and 21 were immunostained for aggrecan and SOX-9, respectively. First, hydrogel slices were obtained following the protocol described in section 7.3.10. For immunoassaying, the slides were washed twice with TBS for 4 min, blocked and permeabilized with $0.1 \% \mathrm{v} / \mathrm{v}$ Tween-20 and $2 \% \mathrm{BSA}$ in TBS during $15 \mathrm{~min}$ at room temperature. After, aggrecan at 1:500 and SOX-9 at 1:250 primary antibodies were diluted in blocking solution and incubated by separately overnight at $4^{\circ} \mathrm{C}$. Samples were washed four times with $0.1 \%$ v/v Tween-20 in TBS for 3 min and aggrecan at 1:300 and SOX-9 at 1:350 secondary antibodies were diluted in blocking solution and 
incubated by separately at $37^{\circ} \mathrm{C}$ during $1 \mathrm{~h}$ in dark. Finally, slides were washed four times with $0.1 \% \mathrm{v} / \mathrm{v}$ Tween-20 in TBS for $3 \mathrm{~min}$ and stained with DAPI (1:500 in PBS $1 \mathrm{X})$ to reveal the nucleus. Images from immunofluorescence were taken using a microscope with integrated camera (Nikon Eclipse 80i, Melville, NY USA).).

A quantification of aggrecan and SOX-9 expression was carried out using the samples stained previously. The total amount of expressed molecules was analyzed using 20X-magnification images $(n=9)$. Aggrecan and SOX-9 positive cells were recognized and counted using image J software (NIH Image software, Bethesda, MD USA).

\subsubsection{Gene expression assay}

Total RNA isolation was carried out for samples at days 14 and 21. Two hydrogels of the same group were mixed and disintegrated by pipetting adding $700 \mu \mathrm{L}$ of QIAzol lysis reagent. Then, $140 \mu \mathrm{L}$ of chloroform were added to the mixture and centrifugated at $13000 \mathrm{rpm}$ for $10 \mathrm{~min}$ at $4^{\circ} \mathrm{C}$. The liquid phase was carefully removed and transferred to nuclease-free tube. After, $140 \mu \mathrm{L}$ of chloroform were added to the RNA and centrifugated at $13000 \mathrm{rpm}$ for $10 \mathrm{~min}$ at $4^{\circ} \mathrm{C}$. Thereafter, the upper aqueous phase was carefully removed and transferred to new nucleasefree tube and a proportion 1:1 of isopropanol was added to the RNA. The sample was stored at $-80^{\circ} \mathrm{C}$ for $30 \mathrm{~min}$, and then centrifugated at $14.000 \mathrm{rpm}$ for another $30 \mathrm{~min}$. The isopropanol was removed and 1 $\mathrm{mL}$ of ethanol for molecular biology (100\%) was added to wash the RNA. The sample was centrifugated at $140.000 \mathrm{rpm}$ for $1 \mathrm{~min}$ and the ethanol was removed from the sample. Finally, the RNA was resuspended in $30 \mu \mathrm{L}$ of Nuclease-Free water and RNA quality was measured at $260 \mathrm{~nm}$ in a UV spectrophotometer Q3000 (Quawell, San Jose, CA, USA).

For reverse transcription of the isolated RNA, mixtures containing 12 $\mu \mathrm{L}$ of RNA, $1 \mu \mathrm{L}$ of dsDNsa Buffer (10X) and $1 \mu \mathrm{L}$ dsDNsa were heated at $37{ }^{\circ} \mathrm{C}$ for $2 \mathrm{~min}$. Then, $4 \mu \mathrm{L}$ of Reaction Mix (5X) and $2 \mu \mathrm{L}$ of Maxima Enzyme Mix were added to the mixtures and heated during 
$10 \mathrm{~min}$ at $25^{\circ} \mathrm{C}$, followed by $15 \mathrm{~min}$ at $50^{\circ} \mathrm{C}$, and finally for $5 \mathrm{~min}$ at $85^{\circ} \mathrm{C}$ in a MJ Mini thermal cycler (Bio-Rad, Hercules, CA, USA).

Expression was assessed by real time PCR using primers for aggrecan, collagen types I and II, SOX-9 and glyceraldehyde 3-phosphate dehydrogenase (GAPDH) (Table 7-2). The gene expression analysis was done in a 7500 Fats Real-Time PCR System (Applied Biosystems, Waltham, MA, USA)

Table 7-2: Forward $(F)$ and Reverse $(R)$ primers for pig targets.

\begin{tabular}{ccc}
\hline Target & \multicolumn{1}{c}{ Primers } & Ref \\
\hline \multirow{2}{*}{ Agg } & F: 5'-TGACAACTTCTTCGCCACTG-3' & \\
& R: 5'-TGATCTCGTACCGGTCCTTC-3' \\
Col IA1 & F: 5'-AAGACATCCCACCAGTCACC-3' \\
& R: 5'-CAGTTCTTGATTTCGTCGCA-3' & [347] \\
Col 2A1 & F: 5'-TGAAAAAGGTGCTCCTGGAC-3' \\
& R: 5'-CCTTCTCATCGAATCCTCCA-3' \\
SOX-9 & F: 5'-AGCTCACCAGACCTTGAGGA-3' \\
& R: 5'-CTTGGAATGTGGGTTCGAGT-3' \\
GAPDH & F: 5'-TGGTGAAGGTCGGAGTGAAC-3' \\
& R: 5'-TGTAGTGGAGGTCAATGAAGG-3' \\
\hline
\end{tabular}

\subsubsection{Statistical analysis}

For the statistical studies, Statgraphics software was used. ShapiroWilks test was used to verify normality of data. Then, one-way ANOVA with Tukey post-test was applied. In addition, Kruskal-Wallis nonparametric test or unpaired two-tailed t-test were performed where applicable.

\subsection{Results}

\subsubsection{HA molecular weight}

The calibration curve of the PEO patterns used to calculate the molecular weights of HA-HMW, HA-LMW and HA-Tyr is shown in Figure 7-1A. The results indicate that higher molecular weights have a higher and narrower peak (Figure 7-1B). The molecular weight of the 
HA-HMW, HA-LMW and HA-Tyr is shown in Figure 7-1C. It is possible to observe a decrease in HA molecular weight after acid degradation process. The HA-HMW showed a molecular weight of 1.187.238 Da, while the HA-LMW evidenced a molecular weight of 272.920. After grafting process with tyramine, HA-Tyr molecular weight was $346.276 \mathrm{Da}$.

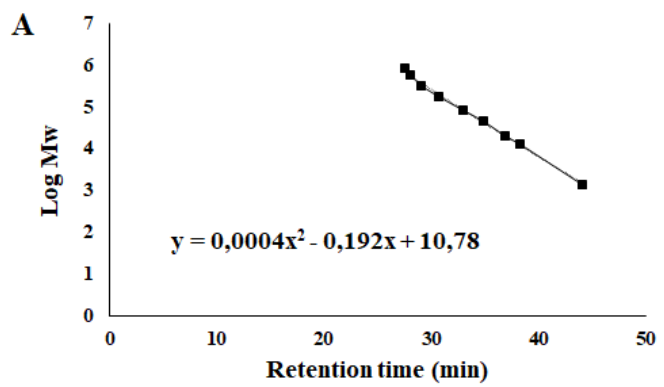

C

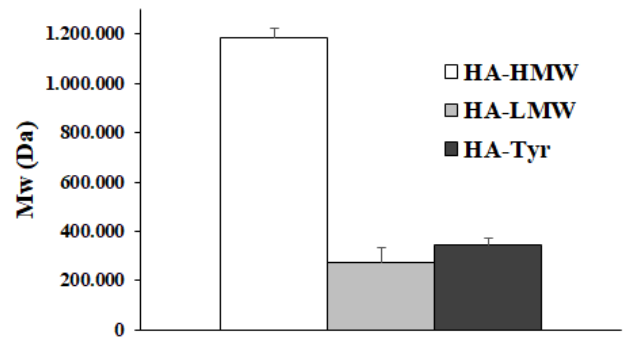

B
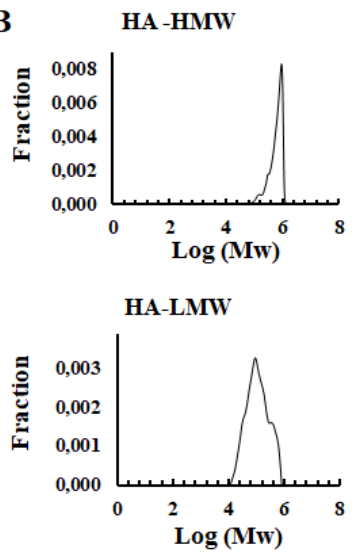

HA-Tyr

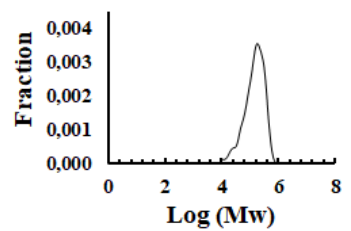

Figure 7-1: Molecular weight of $\mathrm{HA}$.

A) Calibration curve obtained from the PEO standards of known molecular weight. B) Molecular weight distribution curve of HAHMW, HA-LMW and HA-Tyr measured by GPC. C) Molecular weight of HA-HMW, HA-LMW and HA-Tyr. Each curve corresponds to the average of three different samples.

\subsubsection{Tyramine substitution degree in HA and GEL}

The substitution degree of tyramine in both HA and gelatin was measured by spectrophotometry (Table 7-3). The substitution degree for HA was $3 E-4 \mathrm{Mol} \mathrm{Tyr} / \mathrm{mg}$, indicating that $7 \%$ of HA carboxyl groups were substituted by phenol groups after tyramine grafting. Regarding the gelatin, results showed a substitution degree of $4.5 E-$ 
$4 \mathrm{Mol} \mathrm{Tyr} / \mathrm{mg}$ for gelatin, indicating a substitution of $37 \%$ of gelatin carboxyl groups. The degree of substitution indicates that the greater the degree of substitution of the tyramine groups in the polymer, the greater the degree of cross-linking of the hydrogel formed.

Table 7-3: Graft tyramine concentration values and substitution degree in the synthesized products.

\begin{tabular}{ccc}
\hline & HA-Tyr & GEL-Tyr \\
\hline Absorbance (a.u) & 0.07 & 0.11 \\
[Tyr] grafted (mg/mL) & 0.03 & 0.04 \\
[Tyr] grafted (mol/mg) & $3 \mathrm{E}-04$ & $4,5 \mathrm{E}-04$ \\
COOH (mol/mg) & $4.0 \mathrm{E}-03$ & $1.20 \mathrm{E}-03$ \\
Substitution degree (\%) & 7 & 37 \\
\hline
\end{tabular}

\subsubsection{Mechanical properties of the hydrogels}

The rheological properties were measured over polymers that were dissolved in two different mediums: CF-KRB and DMEM, respectively. The variation of the storage modulus $\left(G^{\prime}\right)$ as a function of reaction time, and the complex storage modulus $\left(\left|G^{*}\right|\right)$ as a function of strain are shown in Figure 7-2. Regarding the $G^{\prime}$, both media showed a similar stabilization time; however, the polymers dissolved in CF-KRB evidenced a higher $G^{\prime}(1141 \mathrm{~Pa})$ in comparison with those dissolved in DMEM ( $\left.G^{\prime}=213 P a\right)$ (Figure 7-2A). A similar behavior was observed in the $\left|G^{*}\right|$, which was higher in hybrids that were dissolved in CF-RKB, and lower in hydrogels dissolved in DMEM (Figure 7-2B).

The frequency-dependent of $\left(G^{\prime}\right)$ and loss modulus $\left(G^{\prime \prime}\right)$ is shown in Figure 7-3. Results indicate that polymers, dissolved and cross-linked in presence of CF-KRB, have a higher elastic contribution in comparison with the hybrids cross-linked using DMEM as solvent (Figure 7-3A). It means that hydrogels with higher $\mathrm{G}^{\prime}$ are less viscous and dissipate less energy. This premise can be corroborated with the values of the $\mathrm{G}^{\prime \prime}$, which are lower than $\mathrm{G}^{\prime}$ (Figure 7-3B). 
A

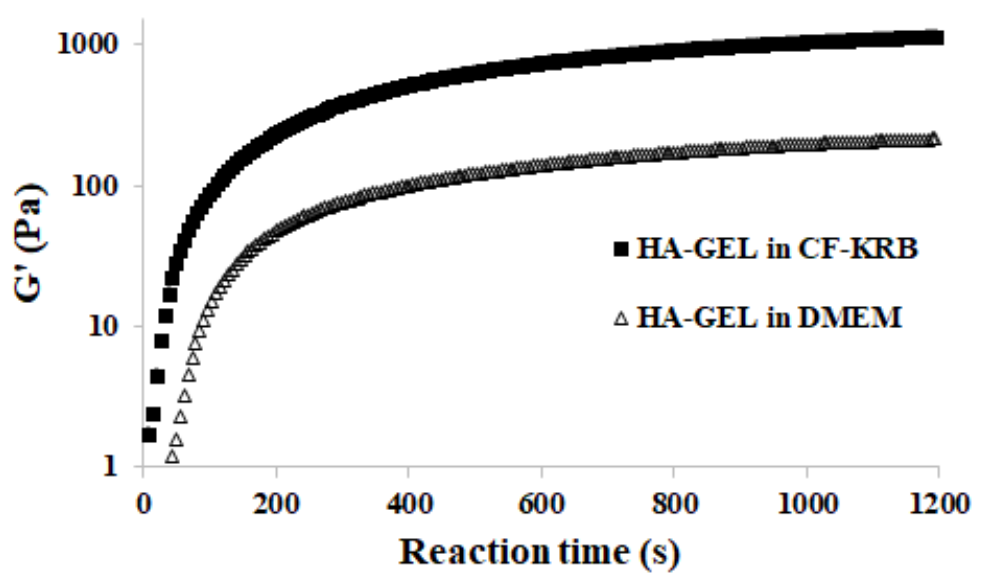

B

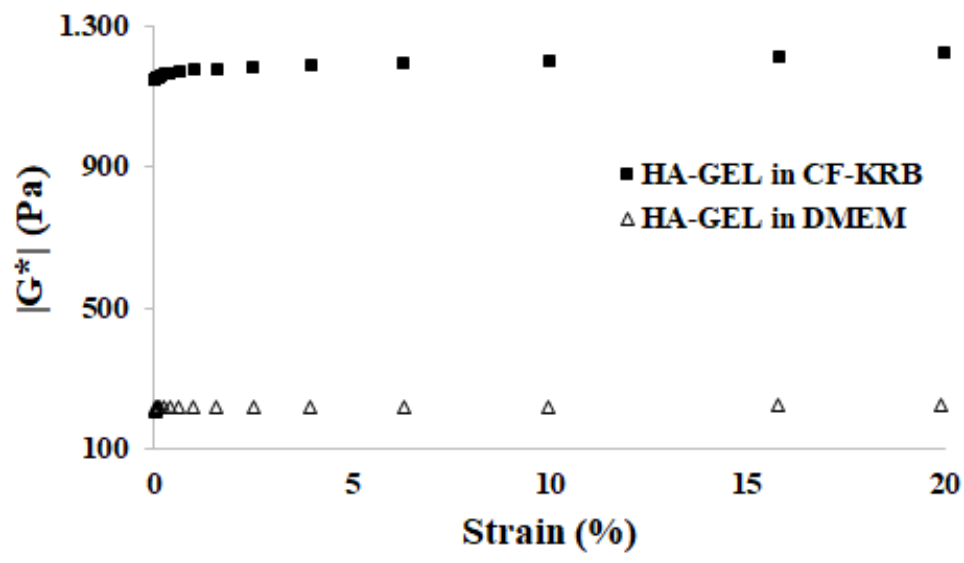

Figure 7-2: Gelation dynamics and strain amplitudes of hydrogels.

A) Oscillatory time sweep to register the crosslinking kinetics of hydrogels. Storage modulus as a function of reaction time. B)

Dynamic strain sweep to calculate the range of strain amplitudes.

Complex modulus magnitude $\left(\left|G^{*}\right|\right)$ of crosslinked hydrogels as a function of the strain. Each curve corresponds to the average of three different samples. 
A

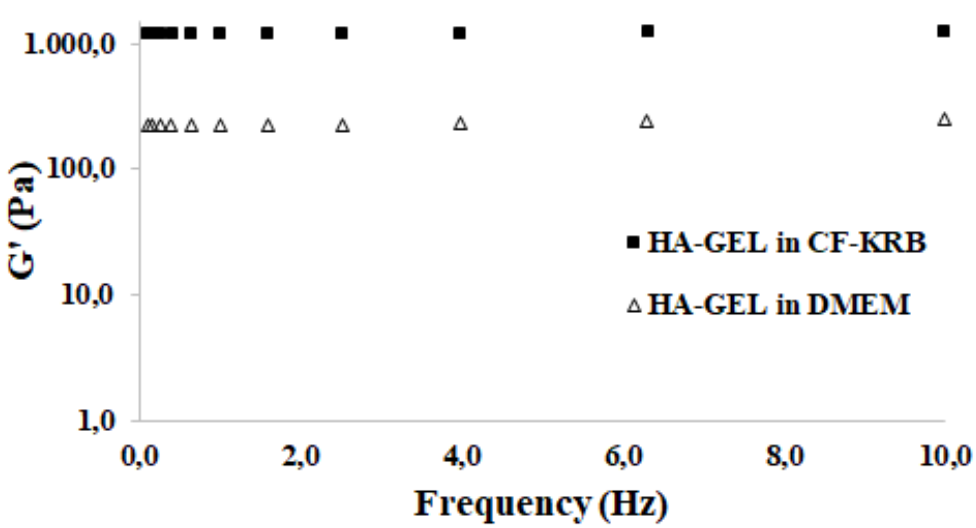

B

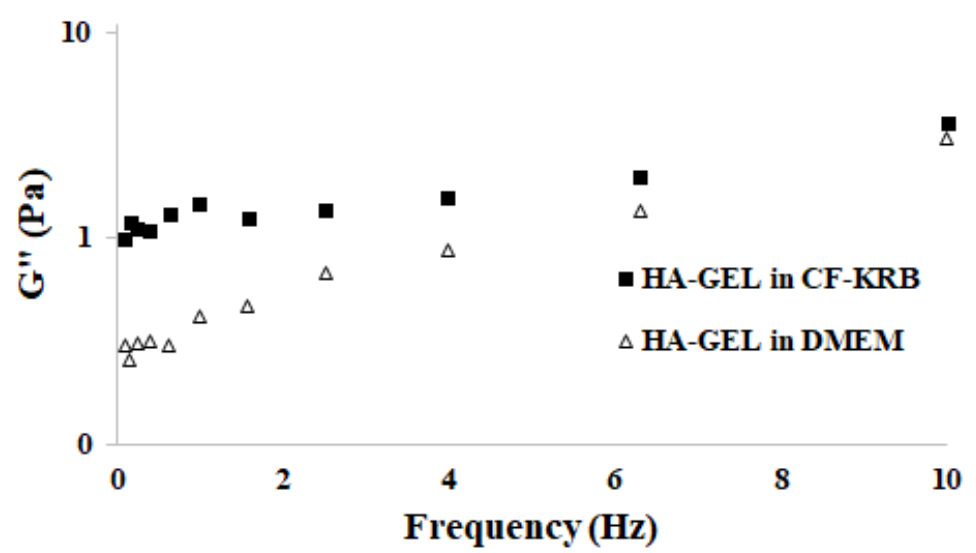

Figure 7-3: Dynamic frequency sweep test to determine the dependence of the dynamic shear modulus and loss factor on the frequency.

A) Storage modulus $\left(G^{\prime}\right)$ B) Loss modulus $\left(G^{\prime \prime}\right)$. Each curve corresponds to the average of three different samples.

\subsection{4}

\section{Electrical properties of the hydrogel}

The permittivity $\left(\varepsilon^{\prime}\right)$ and conductivity $(\sigma)$ of the hydrogels and the DMEM are shown in Figure 7-4. Regarding the $\varepsilon^{\prime}$, which is the ability of a medium to store an EF, it was observed that the DMEM presented 
a higher $\varepsilon^{\prime}=2.67 E+04$ in comparison with the hydrogel $\left(\varepsilon^{\prime}=\right.$ $8.03 E+03)$ (Figure 7-4A). It means that the DMEM yield less electric flux than the hydrogels; therefore, the EF generated by the capacitively system will be higher within the hydrogels.
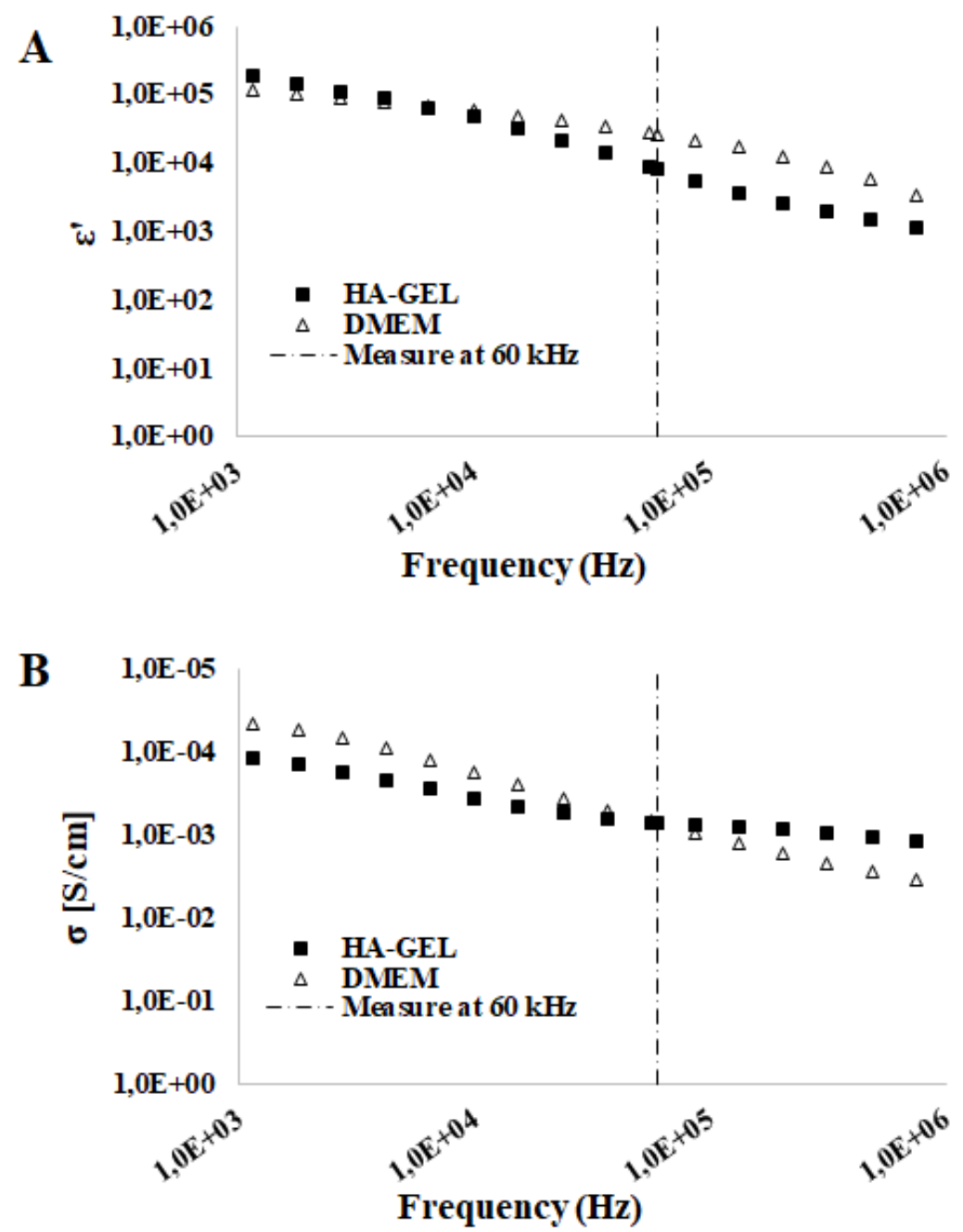

Figure 7-4: Dielectric properties of the hydrogels and culture medium.

A) Permittivity $\left(\varepsilon^{\prime}\right)$ of hydrogels and culture medium. B) Conductivity $(\sigma)$ of hydrogels and culture medium. Each curve corresponds to the average of three different samples. 
Concerning the $\sigma$, it was observed that both the hydrogels and the DMEM presented similar $\sigma$ values (Figure 7-4B). This result indicates that both the polymers and the medium have the same ability to let electric current pass through it.

\subsubsection{Distribution of EFs}

An axisymmetric computational simulation was carried out in order to calculate the EFs in the capacitively coupled system (Figure 7-5).
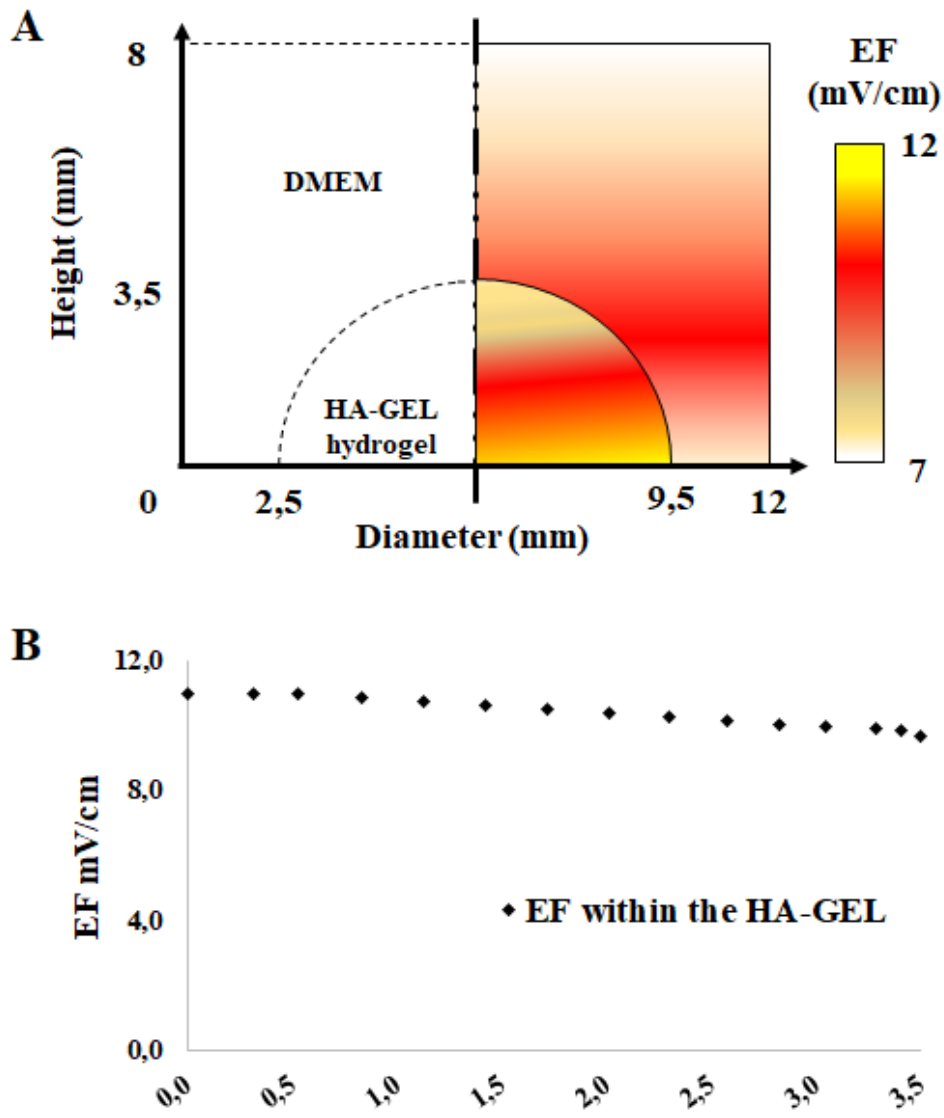

Height mm

Figure 7-5: Distribution of the EFs within the cell culture.

A) Distribution of the EFs within the hydrogel and the DMEM. B)

Values of the EFs within the hydrogel. 
The EF distribution in both the hydrogel and the DMEM is shown in Figure 7-5A. Considering that the cells are immersed within the hydrogel, it is relevant to know the EF intensities within the whole surface of the hybrid. For this reason, in Figure 7-5B it is possible to observe that the EFs within the hydrogels are between 9 and $12 \mathrm{mV} / \mathrm{cm}$.

\subsubsection{Morphology and cell proliferation}

Considering that chondrocyte differentiation is usually observed from day 14 of culture, especially at day 21 , a fluorescence assay with DAPI/actin was performed at these days in order to stain cytoskeleton and identify cell morphology. Results indicated that at days 14 and 21 the cells keep their rounded morphology, except some cells that acquired an elongated shape, eventually indicating that chondrogenesis is favored in the cells encapsulated in the hydrogels (Figure 7-6).
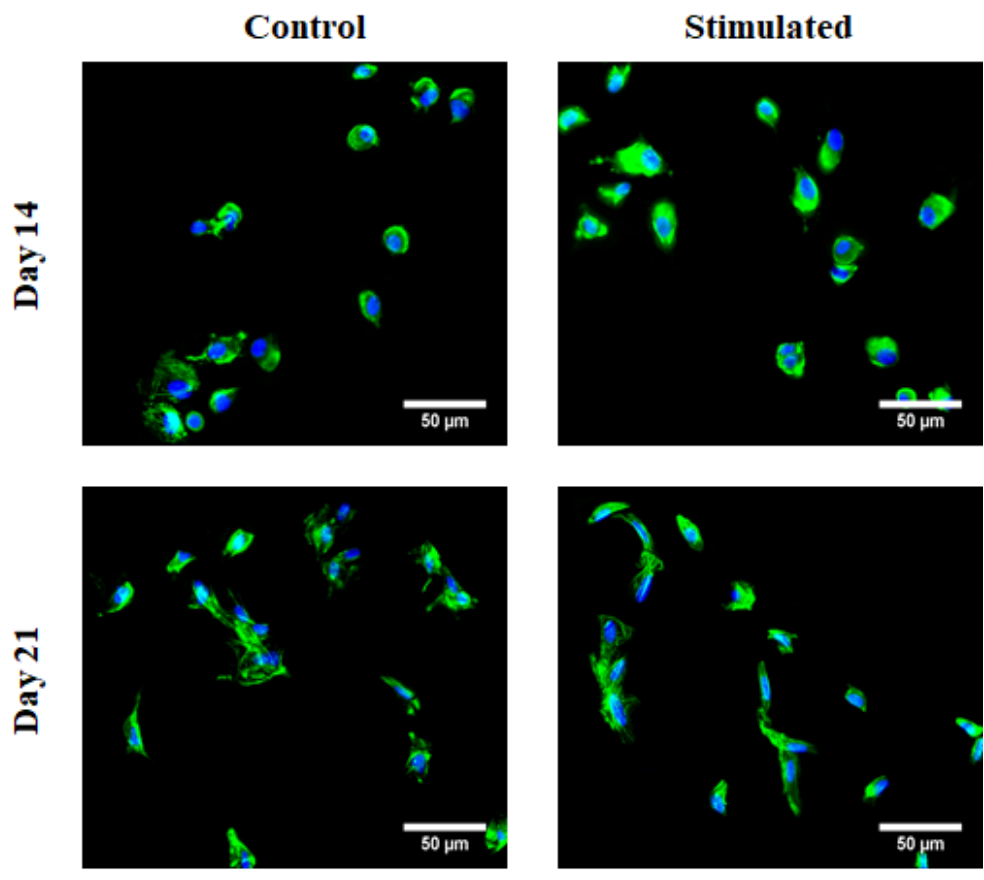

DAPI/actin

Figure 7-6: DAPI/actin staining to control and stimulated hydrogels at days 14 and 21 of culture. Scale bar $=50 \mu \mathrm{m}$. 
Morphology of both controls and stimulated hydrogels was monitored during the whole stimulation period by photos using an inverted microscope. Photos evidenced that at days 2 and 7 both controls and stimulated cultures have a rounded cell morphology (Figure 7-7).
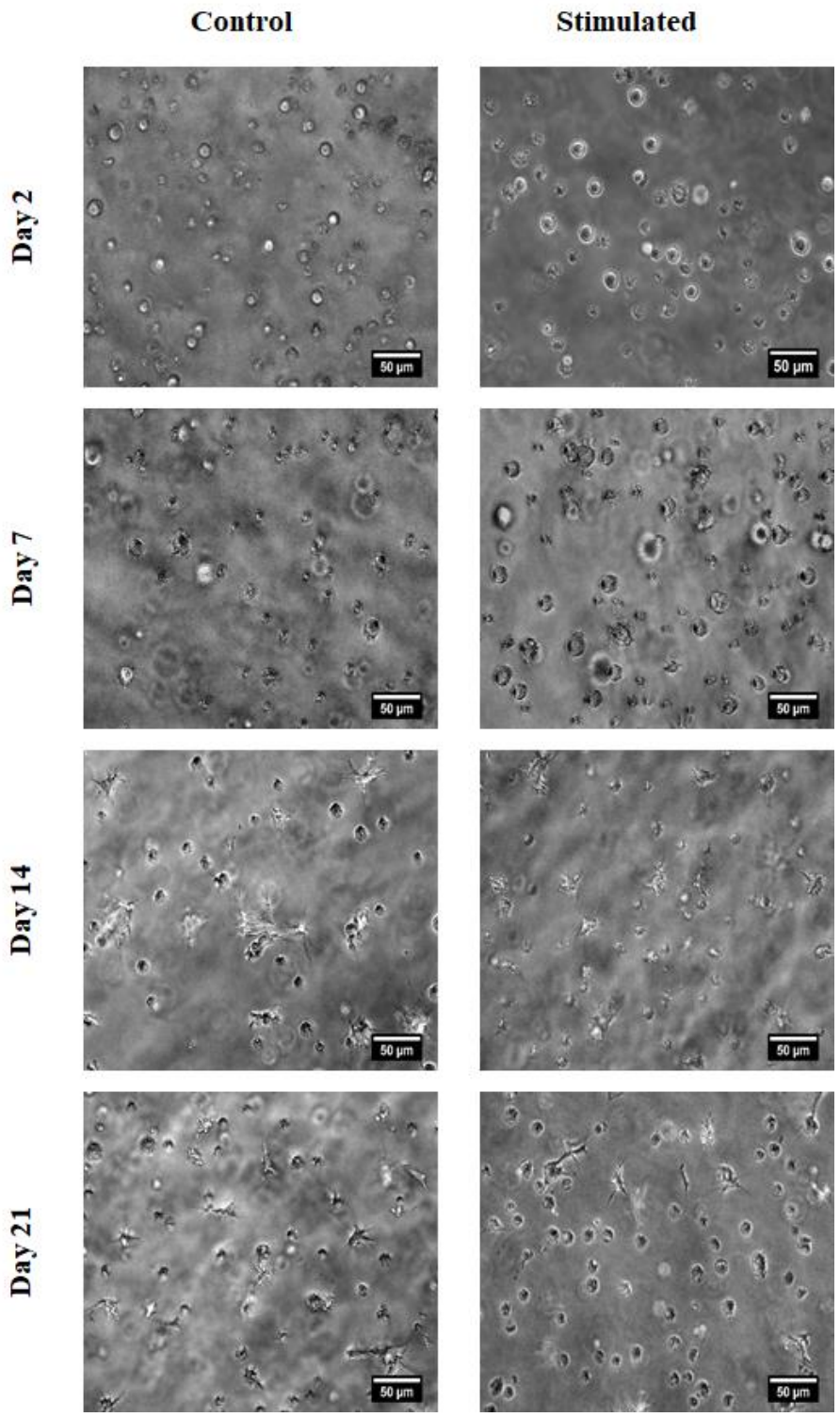

Figure 7-7: Phase contrast images of control and stimulated hydrogels at days 2, 7, 14 and 21 of culture. Scale bar $=50 \mu \mathrm{m}$. 
Regarding cell proliferation, the controls and stimulated cultures showed an exponential growth during the 21 days of culture (Figure 7-8). At days 2 and 7 of culture the controls evidenced a higher proliferation rate in comparison with the stimulated hydrogels. On the contrary, at day 14 the cell proliferation was higher in the stimulated hybrids in comparison with controls. No significant differences were found between controls and stimulated hydrogels at day 21 .

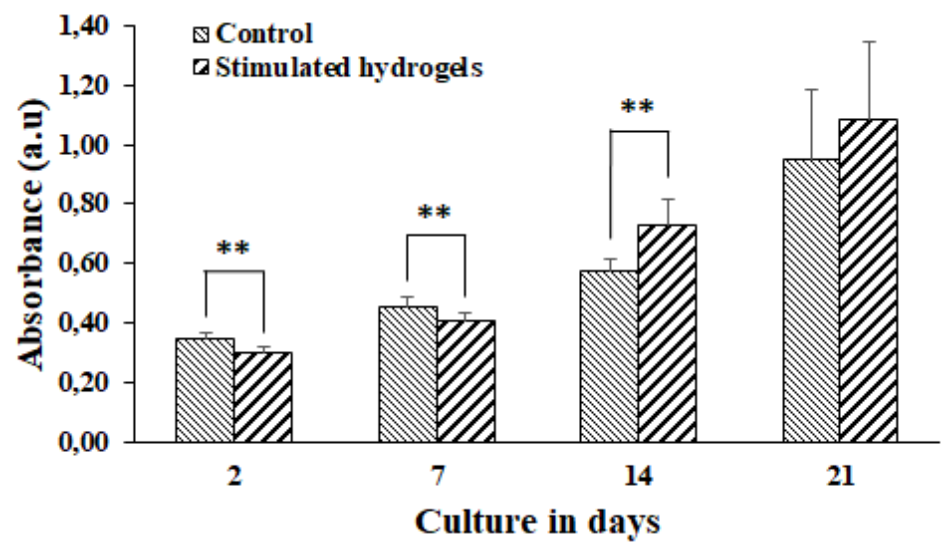

Figure 7-8: Cell proliferation of controls and stimulated hydrogels.

Statistically significant differences were found at days 2, 7 and 14 . $\left(n=5, p<0.05^{* *}\right)$. The results from absorbance measurement are expressed as arbitrary units (aqua).

\subsubsection{Chondrogenic differentiation}

Chondrogenic differentiation was assessed by immunofluorescence at days 14 and 21. Results indicated that the MSCs cultured in the hydrogels and in presence of GM, expressed both SOX-9 and aggrecan at days 14 and 21 (Figure 7-9 and Figure 7-10). Accordingly, the immunofluorescence images allowed to identify the zones in control cultures where there was no expression of aggrecan after 21 days of culture (Figure 7-10 yellow arrows). Although a lack of aggrecan expression in stimulated cultures also was evidenced, this was not as evident as in the control groups. Concerning SOX-9, the expression in both controls and stimulated hydrogels was similar in the immunofluorescence images. 

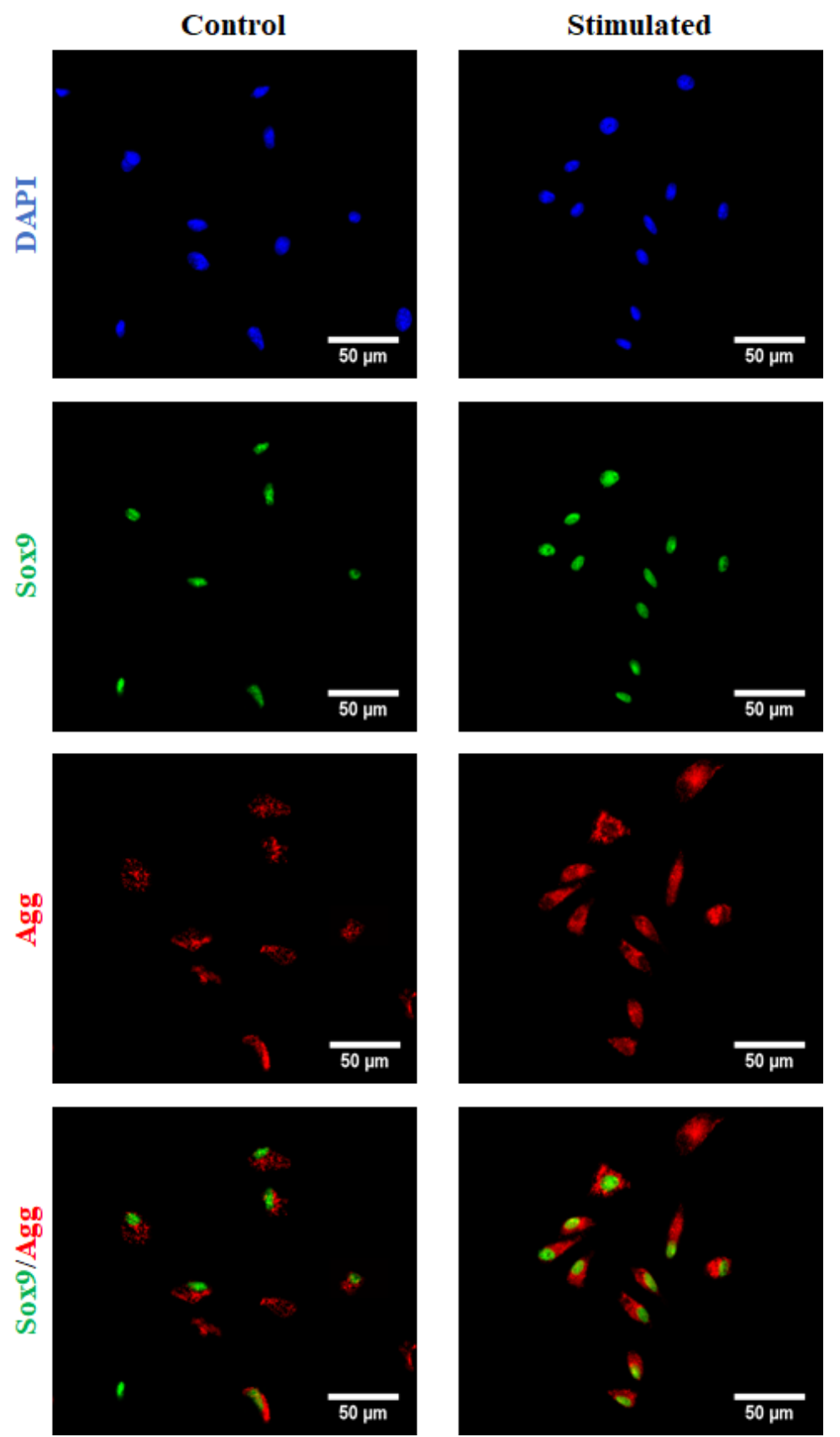

Figure 7-9: Immunofluorescence images for SOX-9 and Aggrecan of MSCs cultured in HA-GEL hydrogels for 14 days. Scale bar $=50$ $\mu \mathrm{m}$. 
Chapter 7. Electric fields on HA - gelatin hydrogels
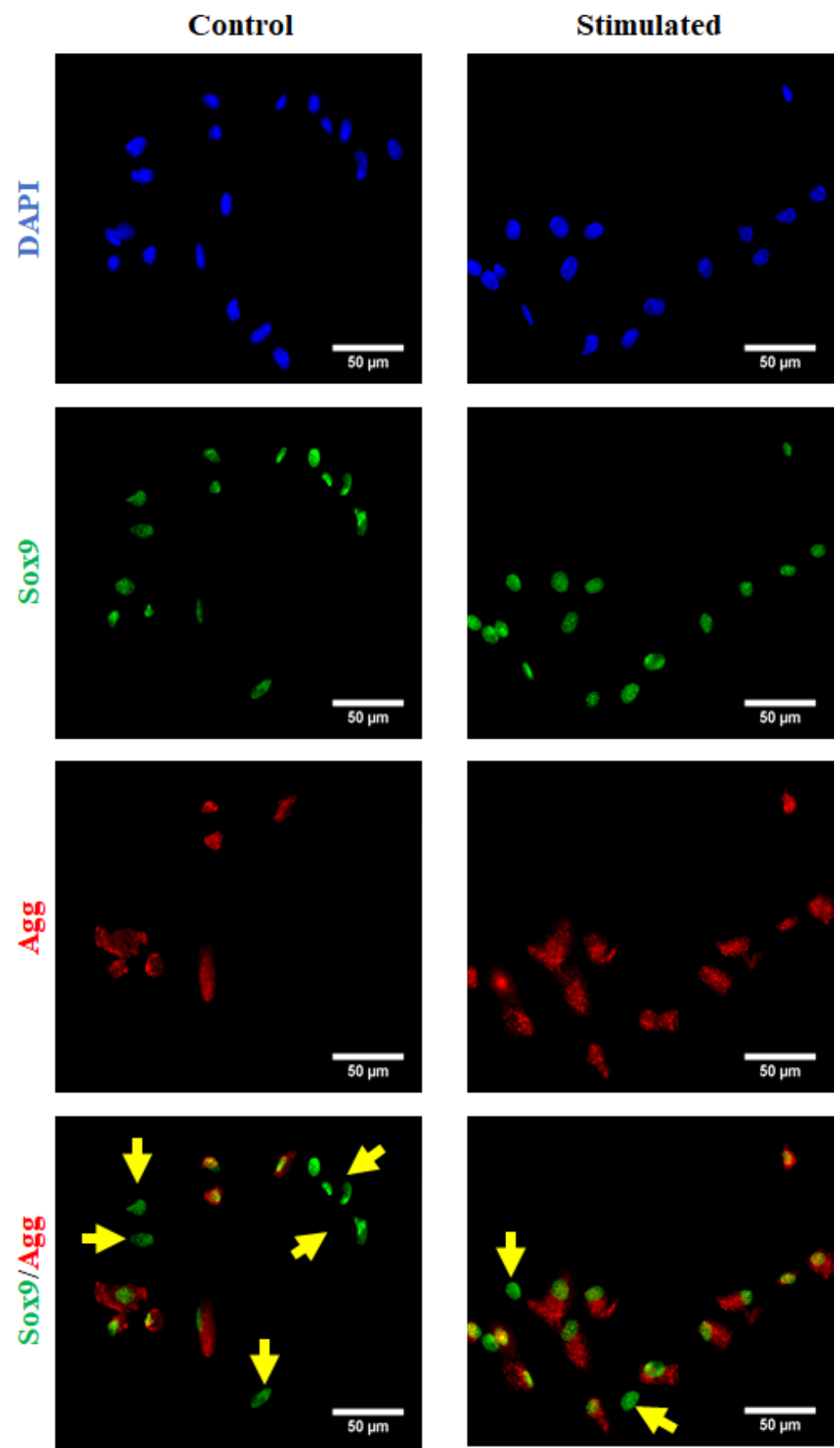

Figure 7-10: Immunofluorescence images for SOX-9 and Aggrecan of MSCs cultured in HA-GEL hydrogels for 21 days. Scale bar $=50$ 

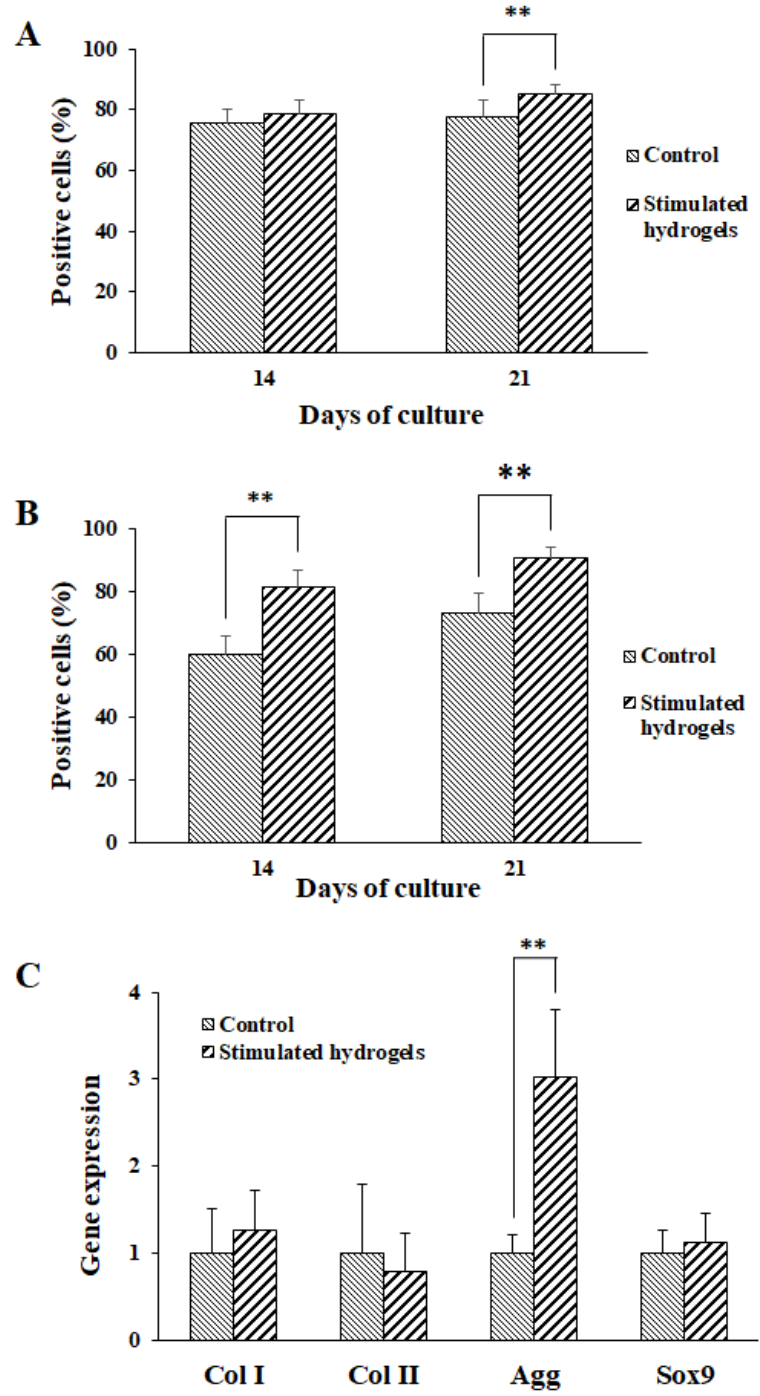

Figure 7-11: Quantification of chondrogenic markers of MSCs encapsulated in the HA-GEL hydrogels.

A) Percentage of positive cells for SOX-9 at days 14 and 21 of culture. Statistically significant differences were found at day 21 ( $\left.p<0.05^{* *}\right)$.

$B)$ Percentage of positive cells for aggrecan at days 14 and 21 of culture. Statistically significant differences were found at days 14 and $21(p<0.05 * *)$. Relative collagen type I and II, aggrecan and SOX-9 gene expression from qPCR. Statistically significant differences were found for aggrecan at day $21(p<0.05 * *)$. 
Moreover, it was observed that both controls and stimulated cultures expressed both molecules. To observe the percentage of cells that were expressing SOX-9 and aggrecan in the immunofluorescence images, a quantification of these chondrogenic markers was performed. Results evidenced that SOX-9 synthesis was higher at day 21 in stimulated cultures compared with controls (Figure 7-11A), while aggrecan expression increased in hydrogels stimulated at days 14 and 21 compared with controls (Figure 7-11B).

The gene expression of controls and stimulated hydrogels is shown in Figure 7-11C. The results showed that there were not significant differences in the expression of collagen type I and II, and SOX-9 between controls and stimulated cultures; however, there was an increase in the expression of aggrecan in hydrogels that were electrically stimulated for 21 days.

\subsection{Discussion}

Different hydrogels and biophysical stimuli have been used to induce the chondrogenic differentiation in order to improve the regenerative properties of MSCs used for cartilage lesions repair [62], [63], [71], [238]. Accordingly, this study id the first attempt to combine HA-GEL injectable hydrogels and electrical stimulation to enhance chondrogenic differentiation of bone marrow derived MSCs in absence of growth factors. The design, characterization and production of hydrogels was focused on develop biomaterials with well-stablished stiffness and dielectric properties for induce chondrogenic differentiation. On the one hand, this study allowed to identify that the stiffness of the hydrogels vary depending on the medium used to dissolve the polymers. It was noticed that polymers dissolved in CF-RKB had higher $G^{\prime}$ in comparison with those dissolved in DMEM. Although there is no evidence about the effect that the DMEM have over the polymers, it has been described that polymers stiffness depend on the cross-linking degree [85]. In this context, we hypothesize that the chemical composition of the DMEM modifies the cross-linking chains of the hydrogels. Therefore, the use a growth medium as solvent leads to hydrogels with a poor reticulated mesh, a less mechanical modulus and unstable during culture. According to these results, the cross-linking 
degree of hydrogels is improved when CF-RKB is used as solvent. Thus, polymers dissolved in this buffer present a higher shear modulus. On the other hand, the dielectric properties of the cell culture medium and the hydrogel were characterized in order to appropriately establish the electrical stimulation conditions. This is the first attempt to calculate the dielectric properties of the HA-GEL hydrogels. Even though a study measured the dielectric constants of a $\mathrm{HA}$ - collagen mixture, the results showed lower $\varepsilon^{\prime}=34.15$ and $\sigma=(1.0 \mathrm{E}-7)$, compared with the $\varepsilon^{\prime}=8.03 \mathrm{E}+03$ and $\sigma=7.10 E-2$ found in this study [348]. This discrepancy may be explained by the concentration of the polymers used to make the hydrogels. The aforementioned study used $0.5 \%(\mathrm{w} / \mathrm{v})$ of HA and $0.5 \%(\mathrm{w} / \mathrm{w})$ of collagen, while the concentration used in this work was $2 \%(\mathrm{w} / \mathrm{v})$ for both HA and gelatin. In this context, it is possible to conclude that higher concentrations of polymer increase the $\varepsilon^{\prime}$ of the hydrogel and in turn enhance the polarization of the molecules to override the internal EF when an external EF is applied. Based on the $\sigma$ found in this work, it is possible to conclude that hydrogels with higher concentration of polymers have low electric resistance, which leads to efficient current flow. In fact, the $\sigma$ of hydrogels depends on the cross-linking degree, as the greater the formation of hydrogen bonds, the greater the density of charge carriers [348]. The mechanical and dielectric properties of the designed hydrogels not only bring novel information about the physicomechanical characteristics of the hybrids, but also allow to understand the MSCs behavior when the scaffolds are being stimulating with EFs.

The HA-GEL injectable hydrogels demonstrated to have great potential as a three-dimensional cell culture scaffolds, as the hybrids stimulated the chondrogenic differentiation in absence of growth factors. This finding is consistent with the results obtained by Moulisová et al., in which they evidenced that mesenchymal stem cells, encapsulated in several ratios of HA-GEL and without growth factors, secretes chondrogenic markers such as aggrecan, SOX-9 and collagen Type II [85]. To our knowledge, few reports in literature have evidenced the use of the mixture HA-GEL hydrogels as a biomaterial for chondrogenesis enhancement. However, it has been demonstrated that similar analogs based either on gelatin or GAGs have been used as matrix supports to 
induce chondrogenesis differentiation. Concerning the gelatin, it has been shown that gelatin hydrogels upregulate the gene expression of SOX-9, collagen type II and GAGs in presence of growth factors [88], [322], [349]. Nevertheless, it was demonstrated that MSCs seeded into gelatin hydrogels acquire a fibroblast-like morphology during culture [88]; therefore, the synthesis of chondrogenic markers is lower in pure gelatin hydrogels in comparison with hybrids that contain hyaluronic acid [85]. Accordingly, in this study a combination between HA and gelatin was chosen in order to provide a hydrated environment with high resistance to shear loading, and with anchoring points to favor cell adhesion. The fibroblast-like morphology acquired by the cells is due to the gelatin content into de hydrogel. It has been observed that pure gelatin hydrogels have more cell adhesive functional groups; therefore, cells have more anchor points and can be easily dispersed within the matrix [322]. In addition, gelatin increases pore size of the hydrogel, which provides more space for cellular elongation, spreading and migration [334]. Regarding the HA hydrogels, it has been demonstrated that HA is a natural polymer that enhances chondrogenic lineage of MScs in presence of growth factors such as TGF- $\beta 3$ [89], [90], [350], which is not adequate for wound healing because during the inflammatory stages it seems to counteract the healing process [351]. Moreover, pure HA hydrogels are materials with very poor cell adhesion properties and low capacity to promote cell proliferation [85]. One of the advantages to use HA in hydrogels is because this polysaccharide and its membrane receptor, CD44, promotes chondrogenesis [264], [352]. Overall, it has been showed that HA mixture with fibrillar proteins may increase the chondrogenic markers expression in basal conditions, due to the fibers provide adhesion properties and polysaccharides bring better mechanical properties [353]. According to this, the hybrids performed in this study are a combination of helical amino acids chains that form native arginineglycine-aspartic acid (RGD) adhesion sites, and consequent chains of disaccharide that provide greater stiffness properties.

Concerning the stimulation method, there are two schemes to apply the EFs to in vitro cultures. The first scheme uses electrodes which are in direct contact with the biological material, while the second scheme 
consists of an indirect coupling system that uses external parallel electrodes. Given that the electrodes that are in contact with culture have insufficient biocompatibility and alter physicochemical features of the cell culture medium [175], it was decided to use an external capacitive system to stimulate the scaffolds. Although the chondrogenic differentiation has been enhanced by the application of EFs using either direct [340], [342] or indirect stimulation [341], [343], the use of external parallel electrodes was implemented in this study because there is evidence that this stimulation method increases cell population and molecular synthesis of different type of cells [175]. Moreover, this noninvasive capacitive coupling system is similar to medical stimulation devices used to treat osteoarthritic knees [177]. In this context, we propose a high-performance electrical device that may be used in the medical industry to enhance the noninvasive therapies focused on the treatment of hyaline cartilage.

Experimental analyzes showed that MSCs behavior is stimulated by EFs of $10 \mathrm{mV} / \mathrm{cm}$ applied for $30 \mathrm{~min}$ each four h per day. A decrease in cell proliferation was observed after 2 and 7 days of stimulation, while an increase cell population was evidenced after 14 days of stimulation compared with controls. This finding contrasts with previous reports, which have not noticed that MSCs cultured in micromass and collagen scaffolds do not proliferate after 7 days of electrical stimulation [341], [343], [354]. However, it has been evidenced that short exposure times of electrical stimulation increased the proliferation rate of MSCs growth in monolayer in presence of chondrogenic medium [340]. In terms of molecular synthesis of MSCs, different chondrogenic markers were used in this study as indicators for chondrogenic differentiation. It was observed that stimulated hydrogels experienced an increase in SOX-9 and aggrecan expression after 14 and 21 days of stimulation. Some literature reports have evidenced that micro-mass cultures of MSCs stimulated with $20 \mathrm{mV} / \mathrm{cm}$ at 1 and 60 $\mathrm{kHz}$ experienced an increase in SOX-9, aggrecan and collagen type II; however, the increase in gene expression was obtained under chondrogenic conditions using TGF- $\beta 3$ [341], [343]. A recent study showed that MSCs encapsulated in bovine collagen-based scaffolds and stimulated with direct coupled EFs for seven days had weaker response regarding the expression of cartilage matrix proteins [354]. These 
results suggest that the stimulated HA-GEL injectable hydrogel here presented is a very good alternative to induce chondrogenic differentiation not only in the absence of exogenous factors, but also in the presence of a biophysical stimulus. This finding evidenced the creation of a novel biocompatible material that can be used not only to treat cartilage injures, but also improve current alternative therapies without causing any undesirable local or systemic effects in the injured zone.

\subsection{Concluding remarks}

The purpose of this study was to carry out a novel framework to enhance the chondrogenic differentiation of MSCs encapsulated into injectable hydrogels and stimulated with external EFs without the need of growth factors. Results showed that EFs affect the proliferation rate and stimulate the synthesis of chondrogenic markers such as SOX-9 and aggrecan. Therefore, the electrical stimulation might be used to improve the cell dynamics of MSCs during in vitro cultures and to enhance therapies such as the autologous implantation of scaffolds in injured cartilaginous tissue. 



\section{CHAPTER 8}

\section{Conclusions and perspectives}



Hyaline cartilage has been extensively studied from different fields such as medicine, physiotherapy and engineering in order to find an alternative therapeutic approach to treat cartilage pathologies. Given the complex morphophysiological structure of cartilaginous tissue, current techniques such as physiotherapeutic treatments, surgical interventions, and biocompatible materials to replace cartilage defects are not definitive therapies to restore cartilage tissue. Nowadays, cartilage tissue engineering has focused on the combination of regenerative medicine techniques with the application of biophysical stimuli to improve the dynamic of the molecular structure of cartilage. Electrical stimulation has been used as external biophysical stimuli to increase chondrocyte proliferation rate and stimulate matrix proteins synthesis. Promising results have been obtained with the application of this kind of stimulation in terms of ECM production enhancement and chondrocyte morphophysiology modulation; however, a definitive treatment either to restore cartilage completely or to replace native cartilage with a biomimetic material with similar biochemical and biomechanical features has not been achieved yet.

The effect of EFs on chondrocytes has been assessed using several methodologies to apply the electric fields, where the most used parameters are the EF intensity, the stimulation times and the period that the biological sample need to be under stimulation. On the other hand, the EFs can be applied in vitro or in vivo using either direct contact electrodes or external capacitively coupled electrodes. However, there are discrepancies in the reported results because parameters such as the stimulation scheme, homogeneity of the EFs and calculation of the EFs are not well documented. Moreover, the methodological differences observed make difficult the comparison and extrapolation of the results obtained with chondrocytes in three most studied scenarios: monolayer cultures, explants and 3D constructs.

Taking this into consideration, this study gives insight in the molecular and morphological features of hyaline cartilage and their cellular behavior when are electrically stimulated. The information obtained from this study allowed the estimation of dielectric constants of the cell culture medium and hydrogels. This characterization was useful to 
analyze and determine computationally the EFs that are stimulating the cells. In addition, the measurement parameters allowed the design and building of the capacitively coupled system that distribute homogeneously the EFs over the entire surface culture.

Initially, the study presented here evaluated the effect of different electrical stimulation schemes using the designed device on chondrocyte monolayer cultures. The results evidenced that cellular behavior was differentially impacted depending on EF intensity and stimulation times per day. In this sense, depending on stimulation scheme we observed in some cases increased proliferation rate, inhibition of the chondrocytes' proliferative capacity or stimulation of GAGs synthesis. These findings are relevant, since if the objective is to increase the cell population, an EF of $4 \mathrm{mV} / \mathrm{cm}$ should be applied during a period of $30 \mathrm{~min}$. But if it is desired to stimulate the synthesis of GAGs, an EF of $8 \mathrm{mV} / \mathrm{cm}$ should be applied during $5 \mathrm{~h}$. Therefore, an adequate electrical stimulation could be carried out to optimize the cellular behavior of chondrocytes during in vitro cultures. Although the results from this study present some limitations, as the chondrocytes cultured in monolayer dedifferentiate into fibroblast-like cells and synthetize non-cartilaginous proteins, this experimental approach served as a proof of concept and allowed the identification of the impact of EFs over chondrocytes. In fact, this finding encourages the development of more detailed experimental studies that consider the application of EFs on environments where the chondrocytes maintain its ideal morphology and express the characteristics molecules that compose the ECM of the hyaline cartilage.

Taking the above mentioned into account, a second experimental approach consisted in the evaluation of the EFs over chondrocytes in a more physiological scenario, therefore the stimulus was applied to ex vivo chondroepiphysis explants. Once again, the results showed that depending on the stimulation scheme used, the morphology of the growth plate chondrocytes and their influence over the epiphyseal plate zones might vary. For instance, EFs of $3.5 \mathrm{mV} / \mathrm{cm}$ applied during $1 \mathrm{~h}$ maintain stable both the chondrocyte morphology and the width growth plate zones after 15 days of stimulation. On the contrary, EFs of 3.5 
$\mathrm{mV} / \mathrm{cm}$ applied during 30 min stimulate chondrocyte hypertrophy and in turn increase the thickness of the Ht-z after 30 days of stimulation. The dynamics of chondrocytes that are in the PH-z can be also be altered by applying EFs of $7 \mathrm{mV} / \mathrm{cm}$ for $1 \mathrm{~h}$, as the columnar arrangement of pre-hypertrophic cells increase, and the growth plate is constituted just by chondrocytes that have increased their volume. Even though a more detailed molecular analysis should be carried out in order to evidence how the biochemical regulation of main may be affected, the information obtained from these experimental procedures opens a new investigative approach that may further explore the mechanisms associated to the observed effects. In fact, it is necessary to analyze the effects of EF stimulation in terms of the biochemical regulation of molecules that control the growth plate function such as PTHrP, Ihh, VEGF, FGFs, BMPs, GAGs and collagens, since a detailed molecular analysis was not assessed in this study.

Finally, and considering that stem cells are been used for cartilage repair, several groups have studied the use of EF to improve chondrogenic differentiation of this cells. In fact, to the best of our knowledge, this work presents the first experience using external EFs stimulation for cultures of MSCs encapsulated into HA-GEL hydrogels without growth factors. Our results showed that this biophysical stimulus has a high-potential to enhance the chondrogenic differentiation of MSCs. The results indicated that EFs of $10 \mathrm{mV} / \mathrm{cm}$ applied for $30 \mathrm{~min}$ four times per day have influence over the proliferation and chondrogenic differentiation. It was possible to observe that the electrical stimulation stimulates aggrecan and sox9 synthesis. Although a stimulation on collagen type II was not evidenced after stimulation, this finding indicates that EFs are a valuable tool to stimulate the production proteoglycans and transcription factors that trigger the signaling pathway characteristic of a native cartilage. Accordingly, the electrical stimulation of MSCs embedded into injectable hydrogels lead to the recognition of some knowledge gaps that need to be accomplished in order to enhance this kind of experimental procedures, and those aspects must be the focus of future works. These include a more detailed analysis quantifying the extracellular protein production in the hydrogel such as total collagen 
and proteoglycans. This kind of analysis will provide useful information in order to create a biomimetic structure that may be applied in early stages of the therapeutic process in cartilage injured zones.

In conclusion, electrical stimulation allows to control the cellular dynamics of chondrocytes and mesenchymal cells either cultured in vitro or within the tissue. This control is achieved using the appropriate input parameters, such as electric field strength, stimulation time per day and culture period. An appropriate combination of these factors can provide desired results such as increased cell population, cellular arrangement, morphological changes of chondrocytes and stimulation of the characteristic molecules of cartilage tissue. In fact, the present work exemplifies the need of characterization of the effect of a specific electrical stimulation with different type of cells, tissues and scaffolds, since although in all scenarios a cellular effect was observed, results vary according to the cell type and its environment. This characterization will allow the use of electrical stimulation as an additional tool to optimize time, resources and money in the therapies that are currently used to recover the hyaline cartilage. 


\section{Bibliography}

[1] A. Bhosale and J. Richardson, "Articular cartilage: structure, injuries and review of management," Br. Med. Bull., vol. 87, no. 1, pp. 77-95, 2008.

[2] J. Vaca-González, M. Gutiérrez, and D. Garzón-Alvarado, "Cartílago articular: estructura, patologías y campos eléctricos como alternativa terapéutica. Revisión de conceptos actuales," Rev. Colomb. Ortop. y Traumatol., vol. 31, no. 4, pp. 202-210, 2017.

[3] T. Ahmed and M. Hincke, "Strategies for articular cartilage lesion repair and functional restoration," Tissue Eng Part B Rev, vol. 16, no. 3, pp. 305-329, 2010.

[4] J. Becerra, J. Andrades, E. Guerado, P. Zamora-Navas, J. LopezPuertas, and A. Reddi, "Articular cartilage: structure and regeneration," Tissue Eng Part B Rev, vol. 16, no. 6, pp. 617$627,2010$.

[5] E. Mackie, L. Tatarczuch, and M. Mirams, "The skeleton: a multi-functional complex organ: the growth plate chondrocyte and endochondral ossification," J Endocrinol, vol. 211, no. 2, pp. 109-121, 2011.

[6] C. O'Conor, N. Case, and F. Guilak, "Mechanical regulation of chondrogenesis," Stem Cell Res Ther, vol. 4, no. 4, p. 61, 2013.

[7] F. Burdan et al., "Morphology and physiology of the epiphyseal growth plate," Folia Histochem Cytobiol, vol. 47, no. 1, pp. 516, 2009.

[8] G. Karsenty, H. Kronenberg, and C. Settembre, "Genetic control of bone formation," Annu Rev Cell Dev Biol, vol. 25, pp. 629648, 2009.

[9] M. Quintero, J. Monfort, and D. Mitrovic, Osteoartrosis: Biología, fosopatología, clínica y tratamiento, Primera. España: Médica Panamericana, 2009.

[10] A. Sophia Fox, A. Bedi, and S. Rodeo, "The basic science of articular cartilage: structure, composition, and function," Sports Health, vol. 1, no. 6, pp. 461-468, 2009.

[11] M. Goldring, "Chondrogenesis, chondrocyte differentiation, and articular cartilage metabolism in health and osteoarthritis," Ther. Adv. Musculoskelet. Dis., vol. 4, no. 4, pp. 269-285, Aug. 2012.

[12] B. Boyce, M. Zuscik, and L. Xing, "Biology of Bone and 
Cartilage," in Genetics of Bone Biology and Skeletal Disease, R. V Thakker, M. P. Whyte, J. A. Eisman, and T. B. T.-G. of B. B. and S. D. (Second E. Igarashi, Eds. Academic Press, 2018, pp. 173-195.

[13] J. Martin and J. Buckwalter, "Articular Cartilage Biology," in Sports Injuries, M. N. Doral, Ed. Berlin, Heidelberg: Springer Berlin Heidelberg, 2012, pp. 685-692.

[14] K. Athanasiou, E. Darling, and J. C. Hu, "Articular Cartilage Tissue Engineering," Synth. Lect. Tissue Eng., vol. 1, no. 1, pp. 1-182, 2009.

[15] A. Ham and D. Cormack, "Bone," in Ham's histology, vol. ninth ed., Philadelphia, USA (1987): Lippincott, 2004.

[16] R. Ballock and R. O'Keefe, "The Biology of the Growth Plate," J. Bone Jt. Surg., vol. 85, no. 4, pp. 715-726, 2003.

[17] G. Kandzierski, Ł. Matuszewski, and A. Wójcik, "Shape of growth plate of proximal femur in children and its significance in the aetiology of slipped capital femoral epiphysis," Int. Orthop., vol. 36, no. 12, pp. 2513-2520, Dec. 2012.

[18] K. Johnson et al., "A Stem Cell-Based Approach to Cartilage Repair," Science (80-. )., vol. 336, no. 6082, pp. 717-721, May 2012.

[19] F. Chen, A. Thomas, J. Hecht, M. Goldring, and J. Lawler, "Cartilage oligomeric matrix protein/thrombospondin 5 supports chondrocyte attachment through interaction with integrins," J. Biol. Chem., vol. 280, no. 38, pp. 32655-32661, Sep. 2005.

[20] F. Chen, K. Rousche, and R. Tuan, "Technology Insight: adult stem cells in cartilage regeneration and tissue engineering," Nat. Clin. Pract. Rheumatol., vol. 2, no. 7, p. 373, Jul. 2006.

[21] H. Kronenberg, "Developmental regulation of the growth plate," Nature, vol. 423, no. 6937, pp. 332-336, 2003.

[22] D. Garzón-Alvarado, M. Roa, and A. Ramírez, "Factores que influyen en el crecimiento endocondral: experimentos y modelos," Rev. Cuba. Ortop. y Traumatol., vol. 22, no. 1, pp. 110, 2008.

[23] H. Akiyama, M. Chaboissier, J. Martin, A. Schedl, and B. de Crombrugghe, "The transcription factor Sox9 has essential roles in successive steps of the chondrocyte differentiation pathway and is required for expression of Sox 5 and Sox6," Genes Dev., vol. 16, no. 21, pp. 2813-2828, Nov. 2002.

[24] S. Stricker, R. Fundele, A. Vortkamp, and S. Mundlos, "Role of Runx Genes in Chondrocyte Differentiation," Dev. Biol., vol. 
245, no. 1, pp. 95-108, 2002.

[25] B. St-Jacques, M. Hammerschmidt, and A. P. McMahon, "Indian hedgehog signaling regulates proliferation and differentiation of chondrocytes and is essential for bone formation," Genes Dev, vol. 13, no. 16, pp. 2072-2086, 1999.

[26] H. Kronenberg, "PTHrP and skeletal development," Ann N Y Acad Sci, vol. 1068, no. 1, pp. 1-13, 2006.

[27] S. Provot and E. Schipani, "Molecular mechanisms of endochondral bone development," Biochem Biophys Res Commun, vol. 328, no. 3, pp. 658-665, 2005.

[28] J. Brouwers, C. van Donkelaar, B. Sengers, and R. Huiskes, "Can the growth factors PTHrP, Ihh and VEGF, together regulate the development of a long bone?," J Biomech, vol. 39, no. 15, pp. 2774-2782, 2006.

[29] Y. Yang, Y. Tan, R. Wong, A. Wenden, L. Zhang, and A. Rabie, "The role of vascular endothelial growth factor in ossification," Int J Oral Sci, vol. 4, no. 2, pp. 64-68, 2012.

[30] C. Maes and G. Carmeliet, "Vascular and Nonvascular Roles of VEGF in Bone Development," in VEGF in Development, New York, NY: Springer New York, 2008, pp. 79-90.

[31] A. Rabie and U. Hagg, "Factors regulating mandibular condylar growth," Am J Orthod Dentofac. Orthop, vol. 122, no. 4, pp. 401-409, 2002.

[32] A. Carreira, F. Lojudice, E. Halcsik, R. Navarro, M. Sogayar, and J. Granjeiro, "Bone Morphogenetic Proteins: Facts, Challenges, and Future Perspectives," J. Dent. Res., vol. 93, no. 4, pp. 335-345, Jan. 2014.

[33] N. Zhou et al., "BMP2 induces chondrogenic differentiation, osteogenic differentiation and endochondral ossification in stem cells," Cell Tissue Res., vol. 366, no. 1, pp. 101-111, 2016.

[34] N. Cohen, R. Foster, and V. Mow, "Composition and Dynamics of Articular Cartilage: Structure, Function, and Maintaining Healthy State," J. Orthop. Sport. Phys. Ther., vol. 28, no. 4, pp. 203-215, Oct. 1998.

[35] S. Poveda-Reyes, "Protein-based injectable hydrogels towards the regeneration of articular cartilage," Universitat Politècnica de València, 2016.

[36] U. Meyer and J. Handschel, "Influence of Biomechanical Loads," in Fundamentals of Tissue Engineering and Regenerative Medicine, 2009, pp. 705-717.

[37] A. Williamson, A. Chen, K. Masuda, E. Thonar, and R. Sah, "Tensile mechanical properties of bovine articular cartilage: 
Variations with growth and relationships to collagen network components," J. Orthop. Res., vol. 21, no. 5, pp. 872-880, Jan. 2006.

[38] B. Elder and K. Athanasiou, "Hydrostatic pressure in articular cartilage tissue engineering: from chondrocytes to tissue regeneration," Tissue Eng Part B Rev, vol. 15, no. 1, pp. 43-53, 2009.

[39] L. Setton, H. Tohyama, and V. Mow, "Swelling and Curling Behaviors of Articular Cartilage," J. Biomech. Eng., vol. 120, no. 3, pp. 355-361, Jun. 1998.

[40] A. Maroudas and P. Bullough, "Permeability of Articular Cartilage," Nature, vol. 219, pp. 1260-1261, Sep. 1968.

[41] Q. Meng et al., "The effect of collagen fibril orientation on the biphasic mechanics of articular cartilage," J. Mech. Behav. Biomed. Mater., vol. 65, pp. 439-453, 2017.

[42] B. Lawless, H. Sadeghi, D. Temple, H. Dhaliwal, D. Espino, and D. Hukins, "Viscoelasticity of articular cartilage: Analysing the effect of induced stress and the restraint of bone in a dynamic environment," J. Mech. Behav. Biomed. Mater., vol. 75, pp. 293-301, Nov. 2017.

[43] L. Li and W. Herzog, "The role of viscoelasticity of collagen fibers in articular cartilage: Theory and numerical formulation," Biorheology, vol. 41, no. 3-4, pp. 181-194, 2004.

[44] N. Parra and D. Garzón-Alvarado, "Phenomenology work setting for articular cartilage damage," Rev Cuba. Ortop Traumatol, vol. 23, no. 2, pp. 1-28, 2009.

[45] R. Lories and F. Luyten, "The bone-cartilage unit in osteoarthritis," Nat Rev Rheumatol, vol. 7, no. 1, pp. 43-49, 2011.

[46] J. Temenoff and A. Mikos, "Review: tissue engineering for regeneration of articular cartilage," Biomaterials, vol. 21, no. 5, pp. 431-440, 2000.

[47] R. Stemberger and K. Kerschan-Schindl, "Osteoarthritis: physical medicine and rehabilitation-nonpharmacological management," Wiener Medizinische Wochenschrift, vol. 163, no. 9-10, pp. 228-235, 2013.

[48] B. Goldring and R. Goldring, "Articular cartilage and subchondral bone in the pathogenesis of osteoarthritis," Ann. N. Y. Acad. Sci., vol. 1192, no. 1, pp. 230-237, Apr. 2010.

[49] M. Mueller and R. Tuan, "Anabolic/Catabolic Balance in Pathogenesis of Osteoarthritis: Identifying Molecular Targets," $P M \& R$, vol. 3, no. 6, pp. 3-11, 2011. 
[50] S. Seed, K. Dunican, and A. Lynch, "Osteoarthritis: a review of treatment options," Geriatrics, vol. 64, no. 10, pp. 20-29, 2009.

[51] B. Diekman and F. Guilak, "Stem cell-based therapies for osteoarthritis: challenges and opportunities," Curr Opin Rheumatol, vol. 25, no. 1, pp. 119-126, 2013.

[52] L. Kuszel, T. Trzeciak, M. Richter, and M. Czarny-Ratajczak, "Osteoarthritis and telomere shortening," J Appl Genet, vol. 56, no. 2, pp. 169-176, 2014.

[53] E. Eriksen and J. Ringe, "Bone marrow lesions: a universal bone response to injury?," Rheumatol. Int., vol. 32, no. 3, pp. 575$584,2012$.

[54] J. Guevara, "Analysis of the mechanical environment within the growth plate during bone development: physiological and pathological implications," Pontificia Universidad Javeriana, Bogotá - Colombia, 2015.

[55] L. Bonafe et al., "Nosology and classification of genetic skeletal disorders: 2015 revision," Am. J. Med. Genet. Part A, vol. 167, no. 12, pp. 2869-2892, Sep. 2015.

[56] M. Coutinho, L. Lacerda, and S. Alves, "Glycosaminoglycan Storage Disorders: A Review," Biochem. Res. Int., pp. 1-16, 2012.

[57] T. McAlindon et al., "OARSI guidelines for the non-surgical management of knee osteoarthritis," Osteoarthr. Cartil., vol. 22, no. 3, pp. 363-388, 2014.

[58] C. Vacanti and J. Vacanti, "Engineering Cartilage and Other Structural Tissues: Principles of Bone and Cartilage Reconstruction," in Principles of Tissue Engineering, R. L. L. Vacanti, Ed. Boston: Academic Press, 2014, pp. 1267-1274.

[59] H. Vasiliadis, J. Wasiak, and G. Salanti, "Autologous chondrocyte implantation for the treatment of cartilage lesions of the knee: a systematic review of randomized studies," Knee Surgery, Sport. Traumatol. Arthrosc., vol. 18, no. 12, pp. 1645$1655,2010$.

[60] M. Hronik-Tupaj and D. Kaplan, "A review of the responses of two- and three-dimensional engineered tissues to electric fields," Tissue Eng Part B Rev, vol. 18, no. 3, pp. 167-180, 2012.

[61] J. Zhu and R. Marchant, "Design properties of hydrogel tissueengineering scaffolds," Expert Rev. Med. Devices, vol. 8, no. 5, pp. 607-626, Sep. 2011.

[62] I. El-Sherbiny and M. Yacoub, "Hydrogel scaffolds for tissue engineering: Progress and challenges," Glob. Cardiol. Sci. 
Pract., no. 3, pp. 316-342, Nov. 2013.

[63] S. Van Vlierberghe, P. Dubruel, and E. Schacht, "BiopolymerBased Hydrogels As Scaffolds for Tissue Engineering Applications: A Review," Biomacromolecules, vol. 12, no. 5, pp. 1387-1408, May 2011.

[64] U. Nöth et al., "Chondrogenic differentiation of human mesenchymal stem cells in collagen type I hydrogels," $J$. Biomed. Mater. Res. Part A, vol. 83A, no. 3, pp. 626-635, May 2007.

[65] A. Yokoyama, I. Sekiya, K. Miyazaki, S. Ichinose, Y. Hata, and T. Muneta, "In vitro cartilage formation of composites of synovium-derived mesenchymal stem cells with collagen gel," Cell Tissue Res., vol. 322, no. 2, pp. 289-298, 2005.

[66] K. Masuoka et al., "Tissue Engineering of Articular Cartilage With Autologous Cultured Adipose Tissue-Derived Stromal Cells Using Atelocollagen Honeycomb-Shaped Scaffold With a Membrane Sealing in Rabbits," J. Biomed. Mater. Res. B. Appl. Biomater., vol. 79B, no. 1, pp. 25-34, 2006.

[67] Y. Li, H. Jia, Q. Cheng, F. Pan, and Z. Jiang, "Sodium alginategelatin polyelectrolyte complex membranes with both high water vapor permeance and high permselectivity," J. Memb. Sci., vol. 375, no. 1, pp. 304-312, 2011.

[68] S. Yang, P. Chen, Y. Chen, and F. Lin, "An In-vitro Study on Regeneration of Human Nucleus Pulposus by Using Gelatin/Chondroitin-6-Sulfate/Hyaluronan Tri-copolymer Scaffold," Artif. Organs, vol. 29, no. 10, pp. 806-814, Sep. 2005.

[69] C. Schanté, G. Zuber, C. Herlin, and T. Vandamme, "Chemical modifications of hyaluronic acid for the synthesis of derivatives for a broad range of biomedical applications," Carbohydr. Polym., vol. 85, no. 3, pp. 469-489, 2011.

[70] F. Lee, J. Chung, and M. Kurisawa, "An injectable enzymatically crosslinked hyaluronic acid-tyramine hydrogel system with independent tuning of mechanical strength and gelation rate," Soft Matter, vol. 4, no. 4, pp. 880-887, 2008.

[71] Y. Li, J. Rodrigues, and H. Tomás, "Injectable and biodegradable hydrogels: gelation, biodegradation and biomedical applications," Chem. Soc. Rev., vol. 41, no. 6, pp. 2193-2221, 2012.

[72] M. Collins and C. Birkinshaw, "Comparison of the effectiveness of four different crosslinking agents with hyaluronic acid hydrogel films for tissue-culture applications," J. Appl. Polym. 
Sci., vol. 104, no. 5, pp. 3183-3191, Mar. 2007.

[73] K. Mahmood, M. Lubowa, A. Fazilah, H. Kamilah, B. Abd Razak, and S. Sulaiman, "Review of Fish Gelatin Extraction, Properties and Packaging Applications," Food Sci. Qual. Manag., vol. 56, pp. 47-59, Nov. 2016.

[74] R. Schrieber and H. Gareis, From Collagen to Gelatine. 2007.

[75] M. da Silva, F. Bode, I. Grillo, and C. Dreiss, "Exploring the Kinetics of Gelation and Final Architecture of Enzymatically Cross-Linked Chitosan/Gelatin Gels," Biomacromolecules, vol. 16, no. 4, pp. 1401-1409, Apr. 2015.

[76] M. Wang, Y. Xia, and S. Wang, "Experimental study on repair of articular cartilage defects with homograft of marrow mesenchymal stem cells seeded onto poly-L-lactic acid/gelatin," Chinese J. reparative Reconstr. Surg., vol. 21, no. 7, pp. 753758, Jul. 2007.

[77] X. Wu et al., "Preparation of aligned porous gelatin scaffolds by unidirectional freeze-drying method," Acta Biomater., vol. 6, no. 3, pp. 1167-1177, 2010.

[78] A. Darr and A. Calabro, "Synthesis and characterization of tyramine-based hyaluronan hydrogels," J. Mater. Sci. Mater. Med., vol. 20, no. 1, pp. 33-44, 2009.

[79] L. Wang, C. Du, J. Chung, and M. Kurisawa, "Enzymatically cross-linked gelatin-phenol hydrogels with a broader stiffness range for osteogenic differentiation of human mesenchymal stem cells," Acta Biomater., vol. 8, no. 5, pp. 1826-1837, 2012.

[80] B. Balakrishnan, M. Mohanty, P. Umashankar, and A. Jayakrishnan, "Evaluation of an in situ forming hydrogel wound dressing based on oxidized alginate and gelatin," Biomaterials, vol. 26, no. 32, pp. 6335-6342, 2005.

[81] O. Ishida, Y. Tanaka, I. Morimoto, M. Takigawa, and S. Eto, "Chondrocytes Are Regulated by Cellular Adhesion Through CD44 and Hyaluronic Acid Pathway," J. Bone Miner. Res., vol. 12, no. 10, pp. 1657-1663, Dec. 2009.

[82] G. Mogoşanu and A. Grumezescu, "Natural and synthetic polymers for wounds and burns dressing," Int. J. Pharm., vol. 463, no. 2, pp. 127-136, 2014.

[83] E. Ruoslahti and M. Pierschbacher, "Arg-Gly-Asp: A versatile cell recognition signal," Cell, vol. 44, no. 4, pp. 517-518, 1986.

[84] B. Sarker et al., "Evaluation of Fibroblasts Adhesion and Proliferation on Alginate-Gelatin Crosslinked Hydrogel," PLoS One, vol. 9, no. 9, pp. 1-12, Sep. 2014.

[85] V. Moulisová, S. Poveda-Reyes, E. Sanmartín-Masiá, L. 
Quintanilla-Sierra, M. Salmerón-Sánchez, and G. Gallego Ferrer, "Hybrid Protein-Glycosaminoglycan Hydrogels Promote Chondrogenic Stem Cell Differentiation," ACS Omega, vol. 2, no. 11, pp. 7609-7620, 2017.

[86] B. Johnstone, T. Hering, A. Caplan, V. Goldberg, and J. Yoo, "In VitroChondrogenesis of Bone Marrow-Derived Mesenchymal Progenitor Cells," Exp. Cell Res., vol. 238, no. 1, pp. 265-272, 1998.

[87] K. Pelttari, E. Steck, and W. Richter, "The use of mesenchymal stem cells for chondrogenesis," Injury, vol. 39, no. 1, pp. 58-65, 2008.

[88] A. Salamon et al., "Gelatin-Based Hydrogels Promote Chondrogenic Differentiation of Human Adipose TissueDerived Mesenchymal Stem Cells In Vitro," Materials (Basel)., vol. 7, no. 2, pp. 1342-1359, Feb. 2014.

[89] C. Chung and J. Burdick, "Influence of Three-Dimensional Hyaluronic Acid Microenvironments on Mesenchymal Stem Cell Chondrogenesis," Tissue Eng. Part A, vol. 15, no. 2, pp. 243-254, 2009.

[90] J. Dvořáková et al., "Chondrogenic differentiation of mesenchymal stem cells in a hydrogel system based on an enzymatically crosslinked tyramine derivative of hyaluronan," J. Biomed. Mater. Res. Part A, vol. 102, no. 10, pp. 3523-3530, Nov. 2013.

[91] P. Angele et al., "Characterization of esterified hyaluronangelatin polymer composites suitable for chondrogenic differentiation of mesenchymal stem cells," J. Biomed. Mater. Res. A, vol. 91, no. 2, pp. 416-427, Nov. 2009.

[92] G. C. Pfeifer et al., "Higher Ratios of Hyaluronic Acid Enhance Chondrogenic Differentiation of Human MSCs in a Hyaluronic Acid-Gelatin Composite Scaffold," Materials, vol. 9, no. 381. pp. 1-15, 2016.

[93] Y. Chen, W. Su, S. Yang, A. Gefen, and F. Lin, "In situ forming hydrogels composed of oxidized high molecular weight hyaluronic acid and gelatin for nucleus pulposus regeneration," Acta Biomater., vol. 9, no. 2, pp. 5181-5193, 2013.

[94] L. Scheuer and S. Black, "The Lower Limb," in The Juvenile Skeleton, San Diego, USA.: Elsevier Science., 2004, pp. 341408.

[95] G. Karsenty, "The complexities of skeletal biology," Nature, vol. 423, no. 6937, pp. 316-318, 2003.

[96] B. Carlson, "Limb development," in Human Embryology and 
Developmental Biology, Elsevier/Saunders, 2013, pp. 193-215.

[97] H. MacLean and H. Kronenberg, "Localization of Indian hedgehog and PTH/PTHrP receptor expression in relation to chondrocyte proliferation during mouse bone development," Dev Growth Differ, vol. 47, no. 2, pp. 59-63, 2005.

[98] J. Mao and H. Nah, "Growth and development: hereditary and mechanical modulations," Am J Orthod Dentofac. Orthop, vol. 125, no. 6, pp. 676-689, 2004.

[99] F. Forriol and F. Shapiro, "Bone development: interaction of molecular components and biophysical forces," Clin Orthop Relat Res, no. 432, pp. 14-33, 2005.

[100] N. Tanaka et al., "Cyclic Mechanical Strain Regulates the PTHrP Expression in Cultured Chondrocytes via Activation of the Ca2+ Channel," J. Dent. Res., vol. 84, no. 1, pp. 64-68, Jan. 2005.

[101] J. Huang, L. Ballou, and K. Hasty, "Cyclic equibiaxial tensile strain induces both anabolic and catabolic responses in articular chondrocytes," Gene, vol. 404, no. 1, pp. 101-109, 2007.

[102] R. Beckmann et al., "Mechanical Forces Induce Changes in VEGF and VEGFR-1/sFlt-1 Expression in Human Chondrocytes," Int. J. Mol. Sci., vol. 15, no. 9, pp. 1545615474, Sep. 2014.

[103] C. Narváez-Tovar and D. Garzón-Alvarado, "Computational modeling of the mechanical modulation of the growth plate by sustained loading," Theor. Biol. Med. Model., vol. 9, no. 41, pp. $1-10,2012$.

[104] D. Garzon-Alvarado, "Can the size of the epiphysis determine the number of secondary ossification centers? A mathematical approach," Comput Methods Biomech Biomed Engin, vol. 14, no. 9, pp. 819-826, 2011.

[105] A. Fasano, M. Herrero, J. Lopez, and E. Medina, "On the dynamics of the growth plate in primary ossification," $J$ Theor Biol, vol. 265, no. 4, pp. 543-553, 2010.

[106] I. Stokes, "Mechanical effects on skeletal growth," $J$ Musculoskelet Neuronal Interact, vol. 2, no. 3, pp. 277-280, 2002.

[107] H. Gray, Anatomy of the Human Body, vol. 20. Philadelphia: Lea \& Febiger, 2000.

[108] D. Carter, T. Orr, D. Fyhrie, and D. Schurman, "Influences of mechanical stress on prenatal and postnatal skeletal development," Clin Orthop Relat Res, vol. 219, no. 1, pp. 237$250,1987$. 
[109] B. van der Eerden, M. Karperien, E. Gevers, C. Lowik, and J. Wit, "Expression of Indian hedgehog, parathyroid hormonerelated protein, and their receptors in the postnatal growth plate of the rat: evidence for a locally acting growth restraining feedback loop after birth," J Bone Min. Res, vol. 15, no. 6, pp. 1045-1055, 2000.

[110] M. Chau et al., "Organization of the Indian hedgehog parathyroid hormone-related protein system in the postnatal growth plate," J. Mol. Endocrinol., vol. 47, no. 1, pp. 99-107, Aug. 2011.

[111] S. Connolly, D. Jaramillo, J. Hong, and F. Shapiro, "Skeletal Development in Fetal Pig Specimens: MR Imaging of Femur with Histologic Comparison," Radiology, vol. 233, no. 2, pp. 505-514, Nov. 2004.

[112] H. Cole, M. Yuasa, G. Hawley, J. Cates, J. Nyman, and J. Schoenecker, "Differential development of the distal and proximal femoral epiphysis and physis in mice," Bone, vol. 52, no. 1, pp. 337-346, 2013.

[113] S. Stevens, G. Beaupre, and D. Carter, "Computer model of endochondral growth and ossification in long bones: biological and mechanobiological influences," J Orthop Res, vol. 17, no. 5, pp. 646-653, 1999.

[114] T. Guo, K. Wang, and M. Tho, "The Influence of Some Biomechanical Factors on Endochondral Ossification on Long Bone," in 6th World Congress of Biomechanics In Conjunction with 14th International Conference on Biomedical Engineering (ICBME) and 5th Asia Pacific Conference on Biomechanics (APBiomech), C. T. Lim and J. C. H. Goh, Eds. Berlin, Heidelberg: Springer Berlin Heidelberg, 2010, pp. 844-847.

[115] S. Shefelbine, C. Tardieu, and D. Carter, "Development of the femoral bicondylar angle in hominid bipedalism," Bone, vol. 30, no. 5, pp. 765-770, 2002.

[116] M. Giorgi, A. Carriero, S. Shefelbine, and N. Nowlan, "Mechanobiological simulations of prenatal joint morphogenesis," J Biomech, vol. 47, no. 5, pp. 989-995, 2014.

[117] D. Garzón-gAlvarado, J. García-Aznar, and M. Doblaré, "A reaction-diffusion model for long bones growth," Biomech Model Mechanobiol, vol. 8, no. 5, pp. 381-395, 2008.

[118] C. van Donkelaar and R. Huiskes, "The PTHrP-Ihh feedback loop in the embryonic growth plate allows PTHrP to control hypertrophy and Ihh to regulate proliferation," Biomech Model Mechanobiol, vol. 6, no. 1-2, pp. 55-62, 2007. 
[119] O. López-Vaca and D. Garzón-Alvarado, "Spongiosa Primary Development: A Biochemical Hypothesis by Turing Patterns Formations," Comput. Math. Methods Med., pp. 1-14, 2012.

[120] D. Garzon-Alvarado, L. Peinado Cortes, and R. Cardenas Sandoval, "A mathematical model of epiphyseal development: hypothesis of growth pattern of the secondary ossification centre," Comput Methods Biomech Biomed Engin, vol. 14, no. 1, pp. 23-32, 2011.

[121] D. Garzon-Alvarado, L. Peinado Cortes, and R. Cardenas Sandoval, "A mathematical model of epiphyseal development: hypothesis on the cartilage canals growth," Comput Methods Biomech Biomed Engin, vol. 13, no. 6, pp. 765-772, 2010.

[122] O. Schatz, E. Golenser, and N. Ben-Arie, "Clearing and photography of whole mount X-gal stained mouse embryos," Biotechniques, vol. 39, no. 5, pp. 650-656, 2005.

[123] B. Redfern, L. David Wise, and S. Spence, "An alternative Alcian Blue dye variant for the evaluation of fetal cartilage," Birth Defects Res. Part B Dev. Reprod. Toxicol., vol. 80, no. 3, pp. 171-176, 2007.

[124] E. Koyama et al., "A distinct cohort of progenitor cells participates in synovial joint and articular cartilage formation during mouse limb skeletogenesis," Dev Biol, vol. 316, no. 1, pp. 62-73, 2008.

[125] L. Peinado Cortés, D. Garzón Alvarado, and R. Cárdenas Sandoval, "Modelado matemático del proceso de formación de los canales de cartílago en la epífisis," Rev. Cuba. Investig. Biomédicas, vol. 28, no. 3, pp. 51-72, 2009.

[126] N. Nowlan, P. Murphy, and P. Prendergast, "A dynamic pattern of mechanical stimulation promotes ossification in avian embryonic long bones," J Biomech, vol. 41, no. 2, pp. 249-258, 2008.

[127] J. Vaal, A. van Soest, and B. Hopkins, "Spontaneous kicking behavior in infants: age-related effects of unilateral weighting," Dev Psychobiol, vol. 36, no. 2, pp. 111-122, 2000.

[128] D. Kelly and P. Prendergast, "Prediction of the Optimal Mechanical Properties for a Scaffold Used in Osteochondral Defect Repair," Tissue Eng., vol. 12, no. 9, pp. 2509-2519, 2006.

[129] D. Garzon-Alvarado, J. Garcia-Aznar, and M. Doblare, "Appearance and location of secondary ossification centres may be explained by a reaction-diffusion mechanism," Comput Biol Med, vol. 39, no. 6, pp. 554-561, 2009. 
[130] J. Murray, "Spatial Pattern Formation with Reaction Diffusion Systems," in Mathematical Biology II: Spatial Models and Biomedical Applications, New York, NY: Springer New York, 2003, pp. 71-140.

[131] V. Nanjundiah, "Alan Turing and "The Chemical Basis of Morphogenesis," in Morphogenesis and Pattern Formation in Biological Systems: Experiments and Models, T. Sekimura, S. Noji, N. Ueno, and P. K. Maini, Eds. Tokyo: Springer Japan, 2003, pp. 33-44.

[132] E. Karimian, A. Chagin, and L. Savendahl, "Genetic regulation of the growth plate," Front Endocrinol, vol. 2, no. 113, pp. 110, 2011.

[133] B. Lanske, M. Amling, L. Neff, J. Guiducci, R. Baron, and H. Kronenberg, "Ablation of the PTHrP gene or the PTH/PTHrP receptor gene leads to distinct abnormalities in bone development," J. Clin. Invest., vol. 104, no. 4, pp. 399-407, 1999.

[134] N. Amizuka, H. Warshawsky, J. Henderson, D. Goltzman, and A. Karaplis, "Parathyroid hormone-related peptide-depleted mice show abnormal epiphyseal cartilage development and altered endochondral bone formation," J. Cell Biol., vol. 126, no. 6, pp. 1611-1623, 1994.

[135] A. Karaplis et al., "Lethal skeletal dysplasia from targeted disruption of the parathyroid hormone-related peptide gene," Genes Dev, vol. 8, no. 3, pp. 277-289, 1994.

[136] B. Gao et al., "A mutation in Ihh that causes digit abnormalities alters its signalling capacity and range," Nature, vol. 458, no. 7242, pp. 1196-1200, 2009.

[137] C. Van Donkelaar, "Effects of Temporal changes in PTHrP and Ihh Expressions on Bone Growth, Studied by a Theoretical Model," in Novel Aspects of PTHrP Physiopathology, vol. 1, C. Luparello, Ed. New York: Nova Science Publishers, 2007, pp. $115-141$.

[138] C. Lee, S. Grad, M. Wimmer, and M. Alini, "The Influence of Mechanical Stimuli on Articular Cartilage Tissue Engineering," in Topics in Tissue Engineering, vol. 2, E. N. A. \& R. L. Reis, Ed. 2006, pp. 1-32.

[139] Z. Hochberg, "Clinical physiology and pathology of the growth plate," Best Pract. Res. Clin. Endocrinol. Metab., vol. 16, no. 3, pp. 399-419, 2002.

[140] J. Stoltz, N. de Isla, C. Huselstein, D. Bensoussan, S. Muller, and V. Decot, "Mechanobiology and cartilage engineering: the 
underlying pathophysiological phenomena," Biorheology, vol. 43, no. 3-4, pp. 171-180, 2006.

[141] H. Yao, Y. Wu, and X. Lu, "Cartilage Mechanobiology," in Mechanobiology Handbook, editor Nagatomi J, Ed. CRC Press, 2011, pp. 229-252.

[142] J. Bleuel, F. Zaucke, G. Brüggemann, and A. Niehoff, "Effects of Cyclic Tensile Strain on Chondrocyte Metabolism: A Systematic Review," PLoS One, vol. 10, no. 3, pp. 2-25, Mar. 2015.

[143] H. Heegaard, S. Beaupré, and R. Carter, "Mechanically modulated cartilage growth may regulate joint surface morphogenesis," J. Orthop. Res., vol. 17, no. 4, pp. 509-517, Feb. 2005.

[144] L. Gray, M. Pizzanelli, J. Grodzinsky, and C. Lee, "Mechanical and physicochemical determinants of the chondrocyte biosynthetic response," J. Orthop. Res., vol. 6, no. 6, pp. 777792, Jul. 2018.

[145] F. Guilak, A. Ratcliffe, and C. Mow Van, "Chondrocyte deformation and local tissue strain in articular cartilage: A confocal microscopy study," J. Orthop. Res., vol. 13, no. 3, pp. 410-421, Jul. 2018.

[146] J. Martel-Pelletier, C. Boileau, J. Pelletier, and P. Roughley, "Cartilage in normal and osteoarthritis conditions," Best Pr. Res Clin Rheumatol, vol. 22, no. 2, pp. 351-384, 2008.

[147] P. Chao, R. Roy, R. Mauck, W. Liu, W. Valhmu, and C. Hung, "Chondrocyte translocation response to direct current electric fields," J Biomech Eng, vol. 122, no. 3, pp. 261-267, 2000.

[148] M. Esposito et al., "Differentiation of human umbilical cordderived mesenchymal stem cells, WJ-MSCs, into chondrogenic cells in the presence of pulsed electromagnetic fields," In Vivo (Brooklyn)., vol. 27, no. 4, pp. 495-500, 2013.

[149] S. Mayer-Wagner et al., "Effects of low frequency electromagnetic fields on the chondrogenic differentiation of human mesenchymal stem cells," Bioelectromagnetics, vol. 32, no. 4, pp. 283-290, 2011.

[150] D. Ciombor, G. Lester, R. Aaron, P. Neame, and B. Caterson, "Low frequency EMF regulates chondrocyte differentiation and expression of matrix proteins," J. Orthop. Res., vol. 20, no. 1, pp. 40-50, 2002.

[151] A. Fioravanti, F. Nerucci, G. Collodel, R. Markoll, and R. Marcolongo, "Biochemical and morphological study of human articular chondrocytes cultivated in the presence of pulsed signal 
therapy," Ann Rheum Dis, vol. 61, no. 11, pp. 1032-1033, 2002.

[152] D. Ciombor, R. Aaron, S. Wang, and B. Simon, "Modification of osteoarthritis by pulsed electromagnetic field--a morphological study," Osteoarthr. Cartil., vol. 11, no. 6, pp. 455-462, 2003.

[153] M. Jahns et al., "The effect of pulsed electromagnetic fields on chondrocyte morphology," Med Biol Eng Comput, vol. 45, no. 10, pp. 917-925, 2007.

[154] K. Zhang, J. Guo, Z. Ge, and J. Zhang, "Nanosecond Pulsed Electric Fields (nsPEFs) Regulate Phenotypes of Chondrocytes through Wnt/ $\beta$-catenin Signaling Pathway," Sci. Rep., vol. 4, no. 5836, pp. 1-8, 2014.

[155] P. Armstrong, C. Brighton, and A. Star, "Capacitively coupled electrical stimulation of bovine growth plate chondrocytes grown in pellet form," J Orthop Res, vol. 6, no. 2, pp. 265-271, 1988.

[156] C. Brighton, L. Jensen, S. Pollack, B. Tolin, and C. Clark, "Proliferative and synthetic response of bovine growth plate chondrocytes to various capacitively coupled electrical fields," J Orthop Res, vol. 7, no. 5, pp. 759-765, 1989.

[157] C. Brighton and P. Townsend, "Increased cAMP production after short-term capacitively coupled stimulation in bovine growth plate chondrocytes," J Orthop Res, vol. 6, no. 4, pp. 552$558,1988$.

[158] C. Brighton, A. Unger, and J. Stambough, "In vitro growth of bovine articular cartilage chondrocytes in various capacitively coupled electrical fields," J Orthop Res, vol. 2, no. 1, pp. 15-22, 1984.

[159] S. Nakasuji, Y. Morita, K. Tanaka, T. Tanaka, and E. Nakamachi, "Effect of pulse electric field stimulation on chondrocytes," in Asian Pacific Conference for Materials and Mechanics, 2009, pp. 1-4.

[160] M. De Mattei et al., "Effects of pulsed electromagnetic fields on human articular chondrocyte proliferation," Connect Tissue Res, vol. 42, no. 4, pp. 269-279, 2001.

[161] C. Brighton, W. Wang, and C. Clark, "Up-regulation of matrix in bovine articular cartilage explants by electric fields," Biochem Biophys Res Commun, vol. 342, no. 2, pp. 556-561, 2006.

[162] C. Brighton, W. Wang, and C. Clark, "The effect of electrical fields on gene and protein expression in human osteoarthritic cartilage explants," J Bone Jt. Surg Am, vol. 90, no. 4, pp. 833- 
848, 2008.

[163] M. De Mattei et al., "Effects of electromagnetic fields on proteoglycan metabolism of bovine articular cartilage explants," Connect Tissue Res, vol. 44, no. 3-4, pp. 154-159, 2003.

[164] L. Macginitie, Y. Gluzband, and A. Grodzinsky, "Electric field stimulation can increase protein synthesis in articular cartilage explants," J. Orthop. Res., vol. 12, no. 2, pp. 151-160, 1994.

[165] A. Ongaro et al., "Chondroprotective effects of pulsed electromagnetic fields on human cartilage explants," Bioelectromagnetics, vol. 32, no. 7, pp. 543-551, 2011.

[166] F. Hilz et al., "Influence of extremely low frequency, low energy electromagnetic fields and combined mechanical stimulation on chondrocytes in 3-D constructs for cartilage tissue engineering," Bioelectromagnetics, vol. 35, no. 2, pp. 116-128, 2014.

[167] W. Wang, Z. Wang, G. Zhang, C. Clark, and C. Brighton, "Upregulation of chondrocyte matrix genes and products by electric fields," Clin Orthop Relat Res, vol. 427, pp. 163-173, 2004.

[168] P. Sadoghi, A. Leithner, R. Dorotka, and P. Vavken, "Effect of pulsed electromagnetic fields on the bioactivity of human osteoarthritic chondrocytes," Orthopedics, vol. 36, no. 3, pp. 360-365, 2013.

[169] B. Cecen et al., "Effects Of Electromagnetic Fields On Chondrocytes Cells Of Human Seeded Onto 3d Collagen-Plla Scaffolds And Chondro-Gide Membrane," Sak. Univ J Sci, vol. 16, no. 3, pp. 213-220, 2012.

[170] M. De Mattei et al., "Effects of physical stimulation with electromagnetic field and insulin growth factor-I treatment on proteoglycan synthesis of bovine articular cartilage," Osteoarthr. Cartil., vol. 12, no. 10, pp. 793-800, 2004.

[171] B. Schmidt-Rohlfing, J. Silny, S. Woodruff, and K. Gavenis, "Effects of pulsed and sinusoid electromagnetic fields on human chondrocytes cultivated in a collagen matrix," Rheumatol Int, vol. 28, no. 10, pp. 971-977, 2008.

[172] V. Nicolin et al., "In vitro exposure of human chondrocytes to pulsed electromagnetic fields," Eur J Histochem, vol. 51, no. 3, pp. 203-212, 2007.

[173] N. Szasz, H. Hung, S. Sen, and A. Grodzinsky, "Electric field regulation of chondrocyte biosynthesis in agarose gel constructs," in 49th Annual Meeting of the Orthopaedic Research Society, 2003, p. 1.

[174] O. Akanji, D. Lee, and D. Bader, "The effects of direct current stimulation on isolated chondrocytes seeded in 3D agarose 
constructs," Biorheology, vol. 45, no. 3-4, pp. 229-243, 2008.

[175] R. Balint, N. Cassidy, and S. Cartmell, "Electrical stimulation: a novel tool for tissue engineering," Tissue Eng Part B Rev, vol. 19, no. 1, pp. 48-57, 2013.

[176] B. Baker, J. Spadaro, A. Marino, and R. Becker, "Electrical stimulation of articular cartilage regeneration," Ann N Y Acad Sci, vol. 238, no. 1, pp. 491-499, 1974.

[177] J. Farr, M. Mont, D. Garland, J. Caldwell, and T. Zizic, "Pulsed electrical stimulation in patients with osteoarthritis of the knee: follow up in 288 patients who had failed non-operative therapy," Surg Technol Int, vol. 15, pp. 227-233, 2006.

[178] J. Vaca-González, J. Guevara, J. Vega, and D. GarzónAlvarado, "An In Vitro Chondrocyte Electrical Stimulation Framework: A Methodology to Calculate Electric Fields and Modulate Proliferation, Cell Death and Glycosaminoglycan Synthesis," Cell. Mol. Bioeng., vol. 9, no. 1, pp. 116-126, 2016.

[179] G. Rodan, L. Bourret, and L. Norton, "DNA synthesis in cartilage cells is stimulated by oscillating electric fields," Science (80-. )., vol. 199, no. 4329, pp. 690-692, 1978.

[180] M. Forgon, V. Vámhidy, and L. Kellényi, "Bone growth accelerated by stimulation of the epiphyseal plate with electric current," Arch. Orthop. Trauma. Surg., vol. 104, no. 2, pp. 121124, 1985.

[181] T. Brighton, B. Pfeffer, and R. Pollack, "In vivo growth plate stimulation in various capacitively coupled electrical fields," $J$. Orthop. Res., vol. 1, no. 1, pp. 42-49, Jul. 1983.

[182] O. Sato and M. Akai, "Effect of direct-current stimulation on the growth plate," Arch. Orthop. Trauma Surg., vol. 109, no. 1, pp. 9-13, 1989.

[183] C. Brighton, G. Pfeffer, and S. Pollack, "In vivo growth plate stimulation in various capacitively coupled electrical fields," $J$. Orthop. Res., vol. 1, no. 1, pp. 42-49, 1983.

[184] M. Fini et al., "Pulsed electromagnetic fields reduce knee osteoarthritic lesion progression in the aged Dunkin Hartley guinea pig," J Orthop Res, vol. 23, no. 4, pp. 899-908, 2005.

[185] M. Fini, G. Giavaresi, A. Carpi, A. Nicolini, S. Setti, and R. Giardino, "Effects of pulsed electromagnetic fields on articular hyaline cartilage: review of experimental and clinical studies," Biomed Pharmacother, vol. 59, no. 7, pp. 388-394, 2005.

[186] H. Nisbet et al., "Effects of electromagnetic field (1.8/0.9 GHz) exposure on growth plate in growing rats," Res. Vet. Sci., vol. 104, pp. 24-29, 2016. 
[187] A. Arsen'ev, M. Dudin, and V. Mikhailov, "Influence of pulsed magnetic field on the bone growth plates in rabbits," Biophysics $(O x f)$., vol. 55, no. 4, pp. 619-622, 2010.

[188] D. Trock, A. Bollet, and R. Markoll, "The effect of pulsed electromagnetic fields in the treatment of osteoarthritis of the knee and cervical spine. Report of randomized, double blind, placebo controlled trials," J Rheumatol, vol. 21, no. 10, pp. 1903-1911, 1994.

[189] D. Trock, A. Bollet, R. Dyer Jr., L. Fielding, W. Miner, and R. Markoll, "A double-blind trial of the clinical effects of pulsed electromagnetic fields in osteoarthritis," J Rheumatol, vol. 20, no. 3, pp. 456-460, 1993.

[190] N. Pipitone and D. Scott, "Magnetic pulse treatment for knee osteoarthritis: a randomised, double-blind, placebo-controlled study," Curr Med Res Opin, vol. 17, no. 3, pp. 190-196, 2001.

[191] P. Nicolakis, J. Kollmitzer, R. Crevenna, C. Bittner, C. Erdogmus, and J. Nicolakis, "Pulsed magnetic field therapy for osteoarthritis of the knee--a double-blind sham-controlled trial," Wien Klin Wochenschr, vol. 114, no. 15-16, pp. 678-684, 2002.

[192] A. Sakai, K. Suzuki, T. Nakamura, T. Norimura, and T. Tsuchiya, "Effects of pulsing electromagnetic fields on cultured cartilage cells," Int Orthop, vol. 15, no. 4, pp. 341-346, 1991.

[193] W. Iannacone, D. Pienkowski, S. Pollack, and C. Brighton, "Pulsing electromagnetic field stimulation of the in vitro growth plate," J. Orthop. Res., vol. 6, no. 2, pp. 239-247, 1988.

[194] K. Sauerland, R. Raiss, and J. Steinmeyer, "Proteoglycan metabolism and viability of articular cartilage explants as modulated by the frequency of intermittent loading," Osteoarthr. Cartil., vol. 11, no. 5, pp. 343-350, 2003.

[195] P. Torzilli et al., "Characterization of cartilage metabolic response to static and dynamic stress using a mechanical explant test system," J Biomech, vol. 30, no. 1, pp. 1-9, 1997.

[196] I. Villemure and I. Stokes, "Growth plate mechanics and mechanobiology. A survey of present understanding," $J$ Biomech, vol. 42, no. 12, pp. 1793-1803, 2009.

[197] I. Stokes, K. Clark, C. Farnum, and D. Aronsson, “Alterations in the growth plate associated with growth modulation by sustained compression or distraction," Bone, vol. 41, no. 2, pp. 197-205, 2007.

[198] A. Niehoff, U. Kersting, F. Zaucke, M. Morlock, and G. Bruggemann, "Adaptation of mechanical, morphological, and biochemical properties of the rat growth plate to dose-dependent 
voluntary exercise," Bone, vol. 35, no. 4, pp. 899-908, 2004.

[199] S. Apte and J. Kenwright, "Physeal distraction and cell proliferation in the growth plate," J Bone Jt. Surg Br, vol. 76, no. 5, pp. 837-843, 1994.

[200] B. Valteau, G. Grimard, I. Londono, F. Moldovan, and I. Villemure, "In vivo dynamic bone growth modulation is less detrimental but as effective as static growth modulation," Bone, vol. 49, no. 5, pp. 996-1004, 2011.

[201] N. Ohashi, A. Robling, D. Burr, and C. Turner, "The Effects of Dynamic Axial Loading on the Rat Growth Plate," J. Bone Miner. Res., vol. 17, no. 2, pp. 284-292, 2002.

[202] J. Ryan, E. Eisner, G. DuRaine, Z. You, and A. Reddi, "Mechanical compression of articular cartilage induces chondrocyte proliferation and inhibits proteoglycan synthesis by activation of the ERK pathway: implications for tissue engineering and regenerative medicine," J Tissue Eng Regen Med, vol. 3, no. 2, pp. 107-116, 2009.

[203] S. Amini, D. Veilleux, and I. Villemure, "Tissue and cellular morphological changes in growth plate explants under compression," J Biomech, vol. 43, no. 13, pp. 2582-2588, 2010.

[204] K. Sergerie, S. Parent, P. Beauchemin, I. Londono, F. Moldovan, and I. Villemure, "Growth plate explants respond differently to in vitro static and dynamic loadings," J Orthop Res, vol. 29, no. 4, pp. 473-480, 2011.

[205] M. Cancel, G. Grimard, D. Thuillard-Crisinel, F. Moldovan, and I. Villemure, "Effects of in vivo static compressive loading on aggrecan and type II and X collagens in the rat growth plate extracellular matrix," Bone, vol. 44, no. 2, pp. 306-315, 2009.

[206] T. Pufe et al., "Mechanical overload induces VEGF in cartilage discs via hypoxia-inducible factor," Am J Pathol, vol. 164, no. 1, pp. 185-192, 2004.

[207] E. Saadat, H. Lan, S. Majumdar, D. Rempel, and K. King, "Long-term cyclical in vivo loading increases cartilage proteoglycan content in a spatially specific manner: an infrared microspectroscopic imaging and polarized light microscopy study," Arthritis Res Ther, vol. 8, no. 5, pp. 1-8, 2006.

[208] E. Pickvance, T. Oegema Jr., and R. Thompson Jr., "Immunolocalization of selected cytokines and proteases in canine articular cartilage after transarticular loading," J Orthop Res, vol. 11, no. 3, pp. 313-323, 1993.

[209] R. Barrett-Jolley, R. Lewis, R. Fallman, and A. Mobasheri, "The Emerging Chondrocyte Channelome," Front. Physiol., vol. 1, 
no. 135 , pp. 1-11, Oct. 2010.

[210] C. Huselstein et al., "No TitleMechanobiology, chondrocyte and cartilage," Biomed. Mater. Eng., vol. 18, no. 4-5, pp. 213-220, 2008.

[211] A. DeLise, L. Fischer, and R. Tuan, "Cellular interactions and signaling in cartilage development," Osteoarthr. Cartil., vol. 8, no. 5, pp. 309-334, 2000.

[212] L. Ding et al., "Mechanical impact induces cartilage degradation via mitogen activated protein kinases," Osteoarthr. Cartil., vol. 18, no. 11, pp. 1509-1517, 2010.

[213] S. Han, W. Wouters, A. Clark, and W. Herzog, "Mechanically induced calcium signaling in chondrocytes in situ," J. Orthop. Res., vol. 30, no. 3, pp. 475-481, Aug. 2011.

[214] R. Madden, S. Han, and W. Herzog, "The effect of compressive loading magnitude on in situ chondrocyte calcium signaling," Biomech Model Mechanobiol, vol. 14, no. 1, pp. 135-142, 2015.

[215] P. Perera et al., "Mechanical signals control SOX-9, VEGF, and c-Myc expression and cell proliferation during inflammation via integrin-linked kinase, B-Raf, and ERK1/2-dependent signaling in articular chondrocytes," Arthritis Res Ther, vol. 12, no. 106, pp. 1-9, 2010.

[216] I. Raizman, J. De Croos, R. Pilliar, and R. Kandel, "Calcium regulates cyclic compression-induced early changes in chondrocytes during in vitro cartilage tissue formation," Cell Calcium, vol. 48, no. 4, pp. 232-242, 2010.

[217] L. Ramage, G. Nuki, and D. Salter, "Signalling cascades in mechanotransduction: cell-matrix interactions and mechanical loading," Scand J Med Sci Sport., vol. 19, no. 4, pp. 457-469, 2009.

[218] D. Lee, T. Noguchi, S. Frean, P. Lees, and D. Bader, "The influence of mechanical loading on isolated chondrocytes seeded in agarose constructs," Biorheology, vol. 37, no. 1-2, pp. 149-161, 2000.

[219] M. Wong, M. Siegrist, and X. Cao, "Cyclic compression of articular cartilage explants is associated with progressive consolidation and altered expression pattern of extracellular matrix proteins," Matrix Biol, vol. 18, no. 4, pp. 391-399, 1999.

[220] J. Steinmeyer and S. Knue, "The proteoglycan metabolism of mature bovine articular cartilage explants superimposed to continuously applied cyclic mechanical loading," Biochem Biophys Res Commun, vol. 240, no. 1, pp. 216-221, 1997.

[221] M. Buschmann, Y. Gluzband, A. Grodzinsky, and E. Hunziker, 
"Mechanical compression modulates matrix biosynthesis in chondrocyte/agarose culture," J Cell Sci, vol. 108, no. 4, pp. 1497-1508, 1995.

[222] N. Farnsworth, L. Antunez, and S. Bryant, "Dynamic compressive loading differentially regulates chondrocyte anabolic and catabolic activity with age," Biotechnol Bioeng, vol. 110, no. 7, pp. 2046-2057, 2013.

[223] N. Nowlan, J. Sharpe, K. Roddy, P. Prendergast, and P. Murphy, "Mechanobiology of embryonic skeletal development: Insights from animal models," Birth Defects Res C Embryo Today, vol. 90, no. 3, pp. 203-213, 2010.

[224] R. Sah, Y. Kim, J. Doong, A. Grodzinsky, A. Plaas, and J. Sandy, "Biosynthetic response of cartilage explants to dynamic compression," J Orthop Res, vol. 7, no. 5, pp. 619-636, 1989.

[225] M. Buschmann, Y. Kim, M. Wong, E. Frank, E. Hunziker, and A. Grodzinsky, "Stimulation of aggrecan synthesis in cartilage explants by cyclic loading is localized to regions of high interstitial fluid flow," Arch Biochem Biophys, vol. 366, no. 1, pp. 1-7, 1999.

[226] L. Bian et al., "Dynamic mechanical loading enhances functional properties of tissue-engineered cartilage using mature canine chondrocytes," Tissue Eng Part A, vol. 16, no. 5, pp. 1781-1790, 2010.

[227] O. Demarteau et al., "Dynamic compression of cartilage constructs engineered from expanded human articular chondrocytes," Biochem Biophys Res Commun, vol. 310, no. 2, pp. 580-588, 2003.

[228] C. Hunter, S. Imler, P. Malaviya, R. Nerem, and M. Levenston, "Mechanical compression alters gene expression and extracellular matrix synthesis by chondrocytes cultured in collagen I gels," Biomaterials, vol. 23, no. 4, pp. 1249-1259, 2002.

[229] C. Hung, R. Mauck, C. Wang, E. Lima, and G. Ateshian, "A paradigm for functional tissue engineering of articular cartilage via applied physiologic deformational loading," Ann Biomed Eng, vol. 32, no. 1, pp. 35-49, 2004.

[230] C. Hunter, J. Mouw, and M. Levenston, "Dynamic compression of chondrocyte-seeded fibrin gels: effects on matrix accumulation and mechanical stiffness," Osteoarthr. Cartil., vol. 12, no. 2, pp. 117-130, 2004.

[231] J. Kisiday, M. Jin, M. DiMicco, B. Kurz, and A. Grodzinsky, "Effects of dynamic compressive loading on chondrocyte 
biosynthesis in self-assembling peptide scaffolds," J. Biomech., vol. 37, no. 5, pp. 595-604, 2004.

[232] P. Wang and W. Tsai, "Modulation of the proliferation and matrix synthesis of chondrocytes by dynamic compression on genipin-crosslinked chitosan/collagen scaffolds," J. Biomater. Sci. Polym. Ed., vol. 24, no. 5, pp. 507-519, Apr. 2013.

[233] E. Lima et al., "The Effect of Applied Compressive Loading on Tissue-Engineered Cartilage Constructs Cultured with TGF\&\#223;3," in 2006 International Conference of the IEEE Engineering in Medicine and Biology Society, 2006, pp. 779782.

[234] E. Lima et al., "The Beneficial Effect of Delayed Compressive Loading on Tissue-Engineered Cartilage Constructs Cultured with TGF- $\beta 3$," Osteoarthritis Cartilage, vol. 15, no. 9, pp. 1025-1033, Sep. 2007.

[235] R. Mauck, S. Seyhan, G. Ateshian, and C. Hung, "Influence of seeding density and dynamic deformational loading on the developing structure/function relationships of chondrocyteseeded agarose hydrogels," Ann Biomed Eng, vol. 30, no. 8, pp. 1046-1056, 2002.

[236] S. Nebelung et al., "Simultaneous anabolic and catabolic responses of human chondrocytes seeded in collagen hydrogels to long-term continuous dynamic compression," Ann. Anat. Anat. Anzeiger, vol. 194, no. 4, pp. 351-358, 2012.

[237] S. Nebelung et al., "No TitlContinuous cyclic compressive loading modulates biological and mechanical properties of collagen hydrogels seeded with human chondrocytes e," Biorheology, vol. 48, no. 5-6, pp. 247-261, 2011.

[238] G. Nicodemus and S. Bryant, "Mechanical loading regimes affect the anabolic and catabolic activities by chondrocytes encapsulated in PEG hydrogels," Osteoarthr. Cartil., vol. 18, no. 1 , pp. 126-137, 2010.

[239] M. Ueki et al., "The Effect of Mechanical Loading on the Metabolism of Growth Plate Chondrocytes," Ann. Biomed. Eng., vol. 36, no. 5, pp. 793-800, 2008.

[240] K. Honda et al., "The effects of high magnitude cyclic tensile load on cartilage matrix metabolism in cultured chondrocytes," Eur. J. Cell Biol., vol. 79, no. 9, pp. 601-609, 2000.

[241] B. Weightman and G. Kempson, "Load carriage," in Adult Articular Cartilage, F. MAR, Ed. London, England, 1979, pp. 293-341.

[242] J. Hu and K. Athanasiou, "The effects of intermittent hydrostatic 
pressure on self-assembled articular cartilage constructs," Tissue Eng, vol. 12, no. 5, pp. 1337-1344, 2006.

[243] J. Suh et al., "Intermittent sub-ambient interstitial hydrostatic pressure as a potential mechanical stimulator for chondrocyte metabolism," Osteoarthr. Cartil., vol. 7, no. 1, pp. 71-80, 1999.

[244] J. Parkkinen et al., "Influence of short-term hydrostatic pressure on organization of stress fibers in cultured chondrocytes," $J$ Orthop Res, vol. 13, no. 4, pp. 495-502, 1995.

[245] M. Lammi et al., "Expression of reduced amounts of structurally altered aggrecan in articular cartilage chondrocytes exposed to high hydrostatic pressure," Biochem J, vol. 304, no. 3, pp. 723730, 1994.

[246] S. Carver and C. Heath, "Increasing extracellular matrix production in regenerating cartilage with intermittent physiological pressure," Biotechnol Bioeng, vol. 62, no. 2, pp. 166-174, 1999.

[247] S. Carver and C. Heath, "Semi-continuous perfusion system for delivering intermittent physiological pressure to regenerating cartilage," Tissue Eng, vol. 5, no. 1, pp. 1-11, 1999.

[248] J. Parkkinen, J. Ikonen, M. Lammi, J. Laakkonen, M. Tammi, and H. Helminen, "Effects of cyclic hydrostatic pressure on proteoglycan synthesis in cultured chondrocytes and articular cartilage explants," Arch Biochem Biophys, vol. 300, no. 1, pp. 458-465, 1993.

[249] D. Duggan, M. Bittner, Y. Chen, P. Meltzer, and J. Trent, "Expression profiling using cDNA microarrays," Nat Genet, vol. 21, no. 1, pp. 10-14, 1999.

[250] G. Ramsay, "DNA chips: state-of-the art," Nat Biotechnol, vol. 16, no. 1, pp. 40-44, 1998.

[251] C. Yeh et al., "Shear stress modulates macrophage-induced urokinase plasminogen activator expression in human chondrocytes," Arthritis Res Ther, vol. 15, no. 2, pp. 1-13, 2013.

[252] R. Smith et al., "Time-dependent effects of intermittent hydrostatic pressure on articular chondrocyte type II collagen and aggrecan mRNA expression," J Rehabil Res Dev, vol. 37, no. 2, pp. 153-161, 2000.

[253] R. Smith et al., "In vitro stimulation of articular chondrocyte mRNA and extracellular matrix synthesis by hydrostatic pressure," J Orthop Res, vol. 14, no. 1, pp. 53-60, 1996.

[254] T. Ikenoue et al., "Mechanoregulation of human articular chondrocyte aggrecan and type II collagen expression by intermittent hydrostatic pressure in vitro," J Orthop Res, vol. 21, 
no. 1, pp. 110-116, 2003.

[255] M. Mohtai et al., "Expression of interleukin-6 in osteoarthritic chondrocytes and effects of fluid-induced shear on this expression in normal human chondrocytes in vitro," J Orthop Res, vol. 14, no. 1, pp. 67-73, 1996.

[256] J. Lee, J. Fitzgerald, M. Dimicco, and A. Grodzinsky, "Mechanical injury of cartilage explants causes specific timedependent changes in chondrocyte gene expression," Arthritis Rheum, vol. 52, no. 8, pp. 2386-2395, 2005.

[257] S. Waldman, C. Spiteri, M. Grynpas, R. Pilliar, and R. Kandel, "Long-term intermittent shear deformation improves the quality of cartilaginous tissue formed in vitro," J Orthop Res, vol. 21, no. 4, pp. 590-596, 2003.

[258] B. Bilgen, D. Chu, R. Stefani, and R. Aaron, "Design of a biaxial mechanical loading bioreactor for tissue engineering," $J$ Vis Exp, vol. 1, no. 74, pp. 1-10, 2013.

[259] Y. Gao et al., "The ECM-Cell Interaction of Cartilage Extracellular Matrix on Chondrocytes," Biomed Res. Int., pp. 18, 2014.

[260] J. Xu, W. Wang, C. Clark, and C. Brighton, "Signal transduction in electrically stimulated articular chondrocytes involves translocation of extracellular calcium through voltage-gated channels," Osteoarthr. Cartil., vol. 17, no. 3, pp. 397-405, 2009.

[261] X. Li and J. Kolega, "Effects of direct current electric fields on cell migration and actin filament distribution in bovine vascular endothelial cells," J Vasc Res, vol. 39, no. 5, pp. 391-404, 2002.

[262] R. Loeser, "Integrins and chondrocyte-matrix interactions in articular cartilage," Matrix Biol., vol. 39, pp. 11-16, Oct. 2014.

[263] S. Kim, J. Turnbull, and S. Guimond, "Extracellular matrix and cell signalling: the dynamic cooperation of integrin, proteoglycan and growth factor receptor," J. Endocrinol., vol. 209, no. 2, pp. 139-151, May 2011.

[264] W. Knudson and R. Loeser, "CD44 and integrin matrix receptors participate in cartilage homeostasis," Cell. Mol. Life Sci., vol. 59, no. 1, pp. 36-44, 2002.

[265] S. Kurtis et al., "Mechanisms of chondrocyte adhesion to cartilage: role of $\beta 1$-integrins, CD44, and annexin V," J. Orthop. Res., vol. 19, no. 6, pp. 1122-1130, Jan. 2006.

[266] W. Knudson, G. Chow, and C. Knudson, "CD44-mediated uptake and degradation of hyaluronan," Matrix Biol., vol. 21, no. 1, pp. 15-23, 2002.

[267] K. von der Mark and J. Mollenhauer, "Annexin V interactions 
with collagen," Cell. Mol. Life Sci. C., vol. 53, no. 6, pp. 539545, 1997.

[268] M. Mycielska and M. Djamgoz, "Cellular mechanisms of directcurrent electric field effects: galvanotaxis and metastatic disease," J Cell Sci, vol. 117, no. 9, pp. 1631-1639, 2004.

[269] M. Moncada, M. Saldarriaga, A. Bravo, and C. Pinedo, "Medición de Impedancia Eléctrica en Tejido Biológico Revisión," Rev. Tecno Lógicas, vol. 1, no. 25, pp. 51-76, 2010.

[270] J. Szafranski, A. Grodzinsky, E. Burger, V. Gaschen, H. Hung, and E. Hunziker, "Chondrocyte mechanotransduction: effects of compression on deformation of intracellular organelles and relevance to cellular biosynthesis," Osteoarthr. Cartil., vol. 12, no. 12, pp. 937-946, 2004.

[271] H. Khayyeri, S. Checa, M. Tägil, F. O'Brien, and P. Prendergast, "Tissue differentiation in an in vivo bioreactor: in silico investigations of scaffold stiffness," J. Mater. Sci. Mater. Med., vol. 21, no. 8, pp. 2331-2336, 2010.

[272] M. Hossain, D. Bergstrom, and X. Chen, "Modelling and simulation of the chondrocyte cell growth, glucose consumption and lactate production within a porous tissue scaffold inside a perfusion bioreactor," Biotechnol. Reports, vol. 5, pp. 55-62, Mar. 2015.

[273] F. Guilak, "Compression-induced changes in the shape and volume of the chondrocyte nucleus," J. Biomech., vol. 28, no. 12, pp. 1529-1541, 1995.

[274] G. Sengers, C. Van Donkelaar, J. Oomens, and T. Baaijens, "Computational Study of Culture Conditions and Nutrient Supply in Cartilage Tissue Engineering," Biotechnol. Prog., vol. 21, no. 4, pp. 1252-1261, Sep. 2008.

[275] B. Cole and M. Malek, Articular Cartilage Lesions: A Practical Guide to Assessment and Treatment, First. New York: Springer, 2004.

[276] D. Rojas and B. Bejarano, "Los usuarios con osteoartrosis de rodilla, UNISALUD, Colombia: una mirada desde la epidemiología crítica," Med. Soc., vol. 5, no. 3, pp. 203-214, 2010.

[277] W. Curl, J. Krome, E. Gordon, J. Rushing, B. Smith, and G. Poehling, "Cartilage injuries: a review of 31,516 knee arthroscopies," Arthroscopy, vol. 13, no. 4, pp. 456-460, 1997.

[278] Y. Yen, B. Cascio, L. O’brien, S. Stalzer, P. Millett, and J. Steadman, "Treatment of Osteoarthritis of the Knee with Microfracture and Rehabilitation," Med. Sci. Sport. Exerc., vol. 
40, no. 2, pp. 200-205, 2008.

[279] I. Sánchez et al., Manual SERMEF de rehabilitación y medicina física, 1st ed. 2006.

[280] S. Redman, S. Oldfield, and C. Archer, "Current strategies for articular cartilage repair," Eur Cell Mater, vol. 9, pp. 23-32, 2005.

[281] U. Meyer, "The History of Tissue Engineering and Regenerative Medicine in Perspective," in Fundamentals of Tissue Engineering and Regenerative Medicine, U. Meyer, J. Handschel, H. P. Wiesmann, and T. Meyer, Eds. Springer Berlin Heidelberg, 2009, pp. 5-12.

[282] H. Breinan, T. Minas, H. Hsu, S. Nehrer, S. Shortkroff, and M. Spector, "Autologous chondrocyte implantation in a canine model: change in composition of reparative tissue with time," $J$ Orthop Res, vol. 19, no. 3, pp. 482-492, 2001.

[283] M. Randall, Y. Shao, L. Wang, and T. Ballock, "Activation of Wnt Planar cell polarity (PCP) signaling promotes growth plate column formation in vitro," J. Orthop. Res., vol. 30, no. 12, pp. 1906-1914, Jun. 2012.

[284] K. Brodkin, A. Garcia, and M. Levenston, "Chondrocyte phenotypes on different extracellular matrix monolayers," Biomaterials, vol. 25, no. 28, pp. 5929-5938, 2004.

[285] E. Mandl, S. Van Der Veen, J. Verhaar, and G. Van Osch, "Multiplication of Human Chondrocytes with Low Seeding Densities Accelerates Cell Yield without Losing Redifferentiation Capacity," Tissue Eng., vol. 10, no. 1-2, pp. 109-118, Jan. 2004.

[286] G. Schulze-Tanzil, "Activation and dedifferentiation of chondrocytes: Implications in cartilage injury and repair," Ann. Anat. - Anat. Anzeiger, vol. 191, no. 4, pp. 325-338, 2009.

[287] R. Coiller, Structures and properties of transmission lines, 3rd ed. The institution of Engineering and Technology, 2007.

[288] Z. Lukacs, "Mucopolysaccharides," in Laboratory Guide to the Methods in Biochemical Genetics, 1st ed., N. Blau., Ed. Heidelberg: Springer, 2008, pp. 287-325.

[289] M. Zhao, H. Bai, E. Wang, J. Forrester, and C. McCaig, "Electrical stimulation directly induces pre-angiogenic responses in vascular endothelial cells by signaling through VEGF receptors," J. Cell Sci., vol. 117, no. 3, pp. 397-405, Jan. 2004.

[290] J. Sun and H. Tan, "Alginate-Based Biomaterials for Regenerative Medicine Applications," Materials (Basel)., vol. 
6, no. 4, pp. 1285-1309, Apr. 2013.

[291] T. Selmi et al., "Autologous chondrocyte implantation in a novel alginate-agarose hydrogel," J. Bone Joint Surg. Br., vol. 90-B, no. 5, pp. 597-604, May 2008.

[292] K. Lee and D. Mooney, "Alginate: properties and biomedical applications," Prog. Polym. Sci., vol. 37, no. 1, pp. 106-126, Jan. 2012.

[293] G. Filardo, E. Kon, A. Roffi, A. Di Martino, and M. Marcacci, "Scaffold-Based Repair for Cartilage Healing: A Systematic Review and Technical Note," Arthrosc. J. Arthrosc. Relat. Surg., vol. 29, no. 1, pp. 174-186, 2013.

[294] E. Mackie, Y. Ahmed, L. Tatarczuch, K. Chen, and M. Mirams, "Endochondral ossification: how cartilage is converted into bone in the developing skeleton," Int J Biochem Cell Biol, vol. 40, no. 1, pp. 46-62, 2008.

[295] M. Moncayo, "Estudio del entorno mecánico en el desarrollo de huesos largos: enfoque computacional y experimental," Universidad Nacional de Colombia, 2014.

[296] Y. Li, M. Ahrens, A. Wu, J. Liu, and A. Dudley, "Calcium/calmodulin-dependent protein kinase II activity regulates the proliferative potential of growth plate chondrocytes," Development, vol. 138, no. 2, pp. 359-370, 2011.

[297] L. Lieben and G. Carmeliet, "The involvement of TRP channels in bone homeostasis," Front. Endocrinol. (Lausanne)., vol. 3, no. 99, pp. 1-9, 2012.

[298] C. Matta, R. Zákány, and A. Mobasheri, "Voltage-Dependent Calcium Channels in Chondrocytes: Roles in Health and Disease," Curr. Rheumatol. Rep., vol. 17, no. 43, pp. 1-11, 2015.

[299] P. Armstrong and C. Brighton, "Failure of the rabbit tibial growth plate to respond to the long-term application of a capacitively-coupled electrical field," J Orthop Res, vol. 4, no. 4, pp. 446-451, 1986.

[300] C. Gabriel, "Compilation of the Dielectric Properties of Body Tissues at RF and Microwave Frequencies.," Environ. Heal., vol. Report No., no. June, p. 21, 1996.

[301] C. Gabriel, S. Gabriel, and E. Corthout, "The dielectric properties of biological tissues: I. Literature survey.," Phys. Med. Biol., vol. 41, no. 11, pp. 2231-49, 1996.

[302] J. J. Vaca-González, J. M. Guevara, J. F. Vega, and D. A. Garzón-Alvarado, "An In Vitro Chondrocyte Electrical 
Stimulation Framework: A Methodology to Calculate Electric Fields and Modulate Proliferation, Cell Death and Glycosaminoglycan Synthesis," Cell. Mol. Bioeng., vol. 9, no. 1, pp. 116-126, 2016.

[303] J. Guevara, H. Castro-Aabril, L. Barrera, and D. GarzónAlvarado, "A quantitative and qualitative growth plate description - a simple framework for chondrocytes columnar arrangement evaluation," J. Mech. Med. Biol., vol. 16, no. 2, pp. $1-15,2016$.

[304] J. M. Guevara, H. A. Castro-Abril, L. A. Barrera, and D. A. Garzón-Alvarado, “A quantitative and qualitative growth plate description - a simple framework for chondrocytes columnar arrangement evaluation," J. Mech. Med. Biol., vol. 16, no. 4, pp. $1-15,2016$.

[305] I. Stokes, P. Mente, J. Iatridis, C. Farnum, and D. Aronsson, "Enlargement of growth plate chondrocytes modulated by sustained mechanical loading," J. Bone Jt. Surg. - Ser. A, vol. 84, no. 10, pp. 1842-1848, 2002.

[306] T. Taghian, D. Narmoneva, and A. Kogan, "Modulation of cell function by electric field: a high-resolution analysis," J. R. Soc. Interface, vol. 12, no. 107, pp. 1-11, 2015.

[307] S. Sundelacruz, M. Levin, and D. Kaplan, "Role of membrane potential in the regulation of cell proliferation and differentiation," Stem Cell Rev. Reports, vol. 5, no. 3, pp. 231246, 2009.

[308] R. van Amerongen and R. Nusse, "Towards an integrated view of Wnt signaling in development," Development, vol. 136, no. 19, pp. 3205-3214, 2009.

[309] C. Lu et al., "Wnt-mediated reciprocal regulation between cartilage and bone development during endochondral ossification," Bone, vol. 53, no. 2, pp. 566-574, 2013.

[310] I. Kim, R. Mauck, and J. Burdick, "Hydrogel design for cartilage tissue engineering: A case study with hyaluronic acid," Biomaterials, vol. 32, no. 34, pp. 8771-8782, 2011.

[311] D. Eyre, "Collagen of articular cartilage. - PubMed - NCBI," Arthritis Res. Ther., vol. 4, no. 1, pp. 30-35, 2002.

[312] C. Knudson and W. Knudson, "Cartilage proteoglycans," Semin. Cell Dev. Biol., vol. 12, no. 2, pp. 69-78, 2001.

[313] J. O'Sullivan, S. D’Arcy, F. Barry, J. Murphy, and C. Coleman, "Mesenchymal chondroprogenitor cell origin and therapeutic potential," Stem Cell Res. Ther., vol. 2, no. 1, pp. 1-7, 2011.

[314] E. George, "Intra-articular hyaluronan treatment for 
osteoarthritis," Ann. Rheum. Dis., vol. 57, no. 11, pp. 637-640, Nov. 1998.

[315] A. Goldberg, K. Mitchell, J. Soans, L. Kim, and R. Zaidi, “The use of mesenchymal stem cells for cartilage repair and regeneration: A systematic review," J. Orthop. Surg. Res., vol. 12, no. 1, pp. 1-30, 2017.

[316] K. Wong, K. Lee, B. Tai, P. Law, E. Lee, and J. Hui, "Injectable cultured bone marrow-derived mesenchymal stem cells in varus knees with cartilage defects undergoing high tibial osteotomy: A prospective, randomized controlled clinical trial with 2 years' follow-up," Arthrosc. - J. Arthrosc. Relat. Surg., vol. 29, no. 12, pp. 2020-2028, 2013.

[317] F. Djouad and R. Tuan, "Mesenchymal Stem Cells: New Insights Into Tissue Engineering and Regenerative Medicine," in Fundamentals of Tissue Engineering and Regenerative Medicine, Springer, 2009, pp. 177-195.

[318] J. Puetzer, J. Petitte, and E. Loboa, "Comparative Review of Growth Factors for Induction of Three-Dimensional In Vitro Chondrogenesis in Human Mesenchymal Stem Cells Isolated from Bone Marrow and Adipose Tissue," Tissue Eng. Part B Rev., vol. 16, no. 4, pp. 435-444, 2010.

[319] Y. Park, M. Sugimoto, A. Watrin, M. Chiquet, and E. Hunziker, "BMP-2 induces the expression of chondrocyte-specific genes in bovine synovium-derived progenitor cells cultured in threedimensional alginate hydrogel," Osteoarthr. Cartil., vol. 13, no. 6, pp. 527-536, 2005.

[320] H. Ma, S. Hung, S. Lin, Y. Chen, and W. Lo, "Chondrogenesis of human mesenchymal stem cells encapsulated in alginate beads," J. Biomed. Mater. Res., vol. 64A, no. 2, pp. 273-281, 2003.

[321] C. Charles-Huang, P. Reuben, G. D'Ippolito, P. Schiller, and H. Cheung, "Chondrogenesis of human bone marrow-derived mesenchymal stem cells in agarose culture," Anat. Rec., vol. 278A, no. 1, pp. 428-436, 2004.

[322] H. Awad, M. Wickham, H. Leddy, J. Gimble, and F. Guilak, "Chondrogenic differentiation of adipose-derived adult stem cells in agarose, alginate, and gelatin scaffolds," Biomaterials, vol. 25, no. 16, pp. 3211-3222, 2004.

[323] R. Mauck, X. Yuan, and R. Tuan, "Chondrogenic differentiation and functional maturation of bovine mesenchymal stem cells in long-term agarose culture," Osteoarthr. Cartil., vol. 14, no. 2, pp. 179-189, 2006. 
[324] J. Zwingmann, A. Mehlhorn, N. Südkamp, B. Stark, M. Dauner, and H. Schmal, "Chondrogenic Differentiation of Human Articular Chondrocytes Differs in Biodegradable PGA/PLA Scaffolds," Tissue Eng., vol. 13, no. 9, pp. 2335-2343, 2007.

[325] L. Freed, J. Marquis, A. Nohria, J. Emmanual, A. Mikos, and R. Langer, "Neocartilage formation in vitro and in vivo using cells cultured on synthetic biodegradable polymers," J. Biomed. Mater. Res., vol. 27, no. 1, pp. 11-23, 1993.

[326] J. Aigner et al., "Cartilage tissue engineering with novel nonwoven structured biomaterial based on hyaluronic acid benzyl ester," J. Biomed. Mater. Res., vol. 42, no. 2, pp. 172$181,1998$.

[327] K. Na et al., "Synergistic effect of TGF $\beta-3$ on chondrogenic differentiation of rabbit chondrocytes in thermo-reversible hydrogel constructs blended with hyaluronic acid by in vivo test," J. Biotechnol., vol. 128, no. 2, pp. 412-422, 2007.

[328] L. Solchaga, J. Temenoff, J. Gao, A. Mikos, A. Caplan, and V. Goldberg, "Repair of osteochondral defects with hyaluronanand polyester-based scaffolds," Osteoarthr. Cartil., vol. 13, no. 4, pp. 297-309, 2005.

[329] P. Levett, F. Melchels, K. Schrobback, D. Hutmacher, J. Malda, and T. Klein, "A biomimetic extracellular matrix for cartilage tissue engineering centered on photocurable gelatin, hyaluronic acid and chondroitin sulfate," Acta Biomater., vol. 10, no. 1, pp. 214-223, 2014.

[330] C. Murphy, A. Matsiko, M. Haugh, J. Gleeson, and F. O'Brien, "Mesenchymal stem cell fate is regulated by the composition and mechanical properties of collagen-glycosaminoglycan scaffolds," J. Mech. Behav. Biomed. Mater., vol. 11, pp. 53-62, 2012.

[331] O. Burnsed, Z. Schwartz, K. Marchand, S. Hyzy, R. OlivaresNavarrete, and B. Boyan, "Hydrogels derived from cartilage matrices promote induction of human mesenchymal stem cell chondrogenic differentiation," Acta Biomater., vol. 43, no. 1, pp. 139-149, 2016.

[332] X. Shu, Y. Liu, F. Palumbo, and G. Prestwich, "Disulfidecrosslinked hyaluronan-gelatin hydrogel films: a covalent mimic of the extracellular matrix for in vitro cell growth," Biomaterials, vol. 24, no. 21, pp. 3825-3834, 2003.

[333] J. Vanderhooft, M. Alcoutlabi, J. Magda, and G. Prestwich, "Rheological Properties of Cross-Linked Hyaluronan-Gelatin Hydrogels for Tissue Engineering," Macromol. Biosci., vol. 9, 
no. 1, pp. 20-28, Dec. 2008.

[334] G. Camci-Unal, D. Cuttica, N. Annabi, D. Demarchi, and A. Khademhosseini, "Synthesis and Characterization of Hybrid Hyaluronic Acid-Gelatin Hydrogels," Biomacromolecules, vol. 14, no. 4, pp. 1085-1092, Feb. 2013.

[335] N. Hwang, M. Kim, S. Sampattavanich, J. Baek, Z. Zhang, and J. Elisseeff, "Effects of Three-Dimensional Culture and Growth Factors on the Chondrogenic Differentiation of Murine Embryonic Stem Cells," Stem Cells, vol. 24, no. 2, pp. 284-291, Aug. 2005.

[336] N. Tandon et al., "Alignment and elongation of human adiposederived stem cells in response to direct-current electrical stimulation," in 2009 Annual International Conference of the IEEE Engineering in Medicine and Biology Society, 2009, pp. 6517-6521.

[337] M. Hronik-Tupaj, W. Rice, M. Cronin-Golomb, D. Kaplan, and I. Georgakoudi, "Osteoblastic differentiation and stress response of human mesenchymal stem cells exposed to alternating current electric fields," Biomed. Eng. Online, vol. 10, no. 1, p. 9, 2011.

[338] Z. Zhao et al., "Directed migration of human bone marrow mesenchymal stem cells in a physiological direct current electric field," Eur. Cells Mater., vol. 22, pp. 344-358, 2011.

[339] T. Banks, P. Luckman, J. Frith, and J. Cooper-White, "Effects of electric fields on human mesenchymal stem cell behaviour and morphology using a novel multichannel device," Integr. Biol., vol. 7, no. 6, pp. 693-712, 2015.

[340] M. Hernández-Bule, C. Paíno, M. Trillo, and A. Úbeda, "Electric stimulation at $448 \mathrm{kHz}$ promotes proliferation of human mesenchymal stem cells," Cell. Physiol. Biochem., vol. 34, no. 5, pp. 1741-1755, 2014.

[341] M. Mardani, S. Roshankhah, B. Hashemibeni, M. Salahshoor, E. Naghsh, and E. Esfandiari, "Induction of chondrogenic differentiation of human adipose-derived stem cells by low frequency electric field," Adv. Biomed. Res., vol. 5, no. 97, pp. $1-7,2016$.

[342] H. Kwon, G. Lee, and H. Chun, "Electrical stimulation drives chondrogenesis of mesenchymal stem cells in the absence of exogenous growth factors," Sci. Rep., vol. 6, no. 39302, pp. 113, 2016.

[343] E. Esfandiari et al., "The effect of high frequency electric field on enhancement of chondrogenesis in human adipose-derived 
stem cells," Iran J Basic Med Sci, vol. 4, no. 3, pp. 571-576, 2014.

[344] S. Poveda-Reyes, V. Moulisova, E. Sanmartín-Masiá, L. Quintanilla-Sierra, M. Salmerón-Sánchez, and G. Gallego Ferrer, "Gelatin - Hyaluronic Acid Hydrogels with Tuned Stiffness to Counterbalance Cellular Forces and Promote Cell Differentiation Gelatin - Hyaluronic Acid Hydrogels with Tuned Stiffness to Counterbalance Cellular Forces and Promote Cell Differentiation," Macromol. Biosci., vol. 16, no. 9, pp. 1311-1324, 2016.

[345] E. Sanmartín-Masiá, S. Poveda-Reyes, and G. Gallego Ferrer, "Extracellular matrix-inspired gelatin/hyaluronic acid injectable hydrogels," Int. J. Polym. Mater. Polym. Biomater., vol. 66, no. 6, pp. 280-288, Apr. 2017.

[346] P. Canto, "Hidrogeles biodegradables para la regeneración de cartílago articular," Universitat Politècnica de València, 2014.

[347] J. Casado et al., "Comparative phenotypic and molecular characterization of porcine mesenchymal stem cells from different sources for translational studies in a large animal model," Vet. Immunol. Immunopathol., vol. 147, no. 1, pp. 104112, 2012.

[348] K. Pietrucha and E. Marzec, "Dielectric properties of the collagen-glycosaminoglycans scaffolds in the temperature range of thermal decomposition," Biophys. Chem., vol. 118, no. 1, pp. 51-56, 2005.

[349] T. Ogawa, T. Akazawa, and Y. Tabata, "In Vitro Proliferation and Chondrogenic Differentiation of Rat Bone Marrow Stem Cells Cultured with Gelatin Hydrogel Microspheres for TGF- $\beta 1$ Release," J. Biomater. Sci. Polym. Ed., vol. 21, no. 5, pp. 609621, Mar. 2010.

[350] S. Ansari et al., "Alginate/hyaluronic acid hydrogel delivery system characteristics regulate the differentiation of periodontal ligament stem cells toward chondrogenic lineage," J. Mater. Sci. Mater. Med., vol. 28, no. 10, p. 162, 2017.

[351] D. Ligi, L. Croce, G. Mosti, J. Raffetto, and F. Mannello, "Chronic Venous Insufficiency: Transforming Growth Factor- $\beta$ Isoforms and Soluble Endoglin Concentration in Different States of Wound Healing," Int. J. Mol. Sci., vol. 18, no. 10, pp. 1-11, Oct. 2017.

[352] W. Knudson and C. B Knudson, "An update on hyaluronan and CD44 in cartilage," Curr. Opin. Orthop., vol. 15, no. 5, pp. 369375, Oct. 2004. 
[353] T. Snyder, K. Madhavan, M. Intrator, R. Dregalla, and D. Park, "A fibrin/hyaluronic acid hydrogel for the delivery of mesenchymal stem cells and potential for articular cartilage repair," J. Biol. Eng., vol. 8, no. 1, p. 10, 2014.

[354] B. Hiemer et al., "Effect of electric stimulation on human chondrocytes and mesenchymal stem cells under normoxia and hypoxia," Mol. Med. Rep., vol. 18, no. 2, pp. 2133-2141, Aug. 2018. 


\section{Appendices}

Appendix A: Value of the parameters used in the simulations herein described.

\begin{tabular}{|c|c|c|c|}
\hline Parameter & Value & Equation & Description \\
\hline$S_{\mathrm{PTHrP}}$ & From 0.9 to 1.1 & $\begin{array}{c}12,13,14, \\
15,20,21 \\
\text { and } 23\end{array}$ & $\begin{array}{c}\text { Concentration of } \\
\text { PTHrP }\end{array}$ \\
\hline$S_{\text {Ihh }}$ & From 0.8 to 1 & $\begin{array}{c}14,15,20 \\
\text { and } 22\end{array}$ & Concentration of Ihh \\
\hline$S_{V E G F}$ & From 0.01 to 0.1 & 19 and 23 & $\begin{array}{c}\text { Concentration of } \\
\text { VEGF }\end{array}$ \\
\hline$D_{\text {PTHrP }}$ & 1 & 14 & $\begin{array}{c}\text { Diffusion constant for } \\
\text { PTHrP }\end{array}$ \\
\hline$D_{\text {Ihh }}$ & 10 & 15 & $\begin{array}{l}\text { Diffusion constant for } \\
\text { Ihh }\end{array}$ \\
\hline$D_{V E G F}$ & 0.1 & 19 & $\begin{array}{l}\text { Diffusion constant for } \\
\text { VEGF }\end{array}$ \\
\hline$\gamma$ & 114 & 12 and 13 & $\begin{array}{l}\text { Rate value for the } \\
\text { reaction term }\end{array}$ \\
\hline$C_{p}$ & 1 & 12 and 13 & $\begin{array}{l}\text { Concentration of } \\
\text { proliferative } \\
\text { chondrocytes }\end{array}$ \\
\hline$\alpha_{1}$ & 0.1 & 12 & \\
\hline$\alpha_{2}$ & 0.9 & 13 & Constant source terms \\
\hline$r$ & 10 & 19 & $\begin{array}{l}\text { Exponential growth } \\
\text { rate of VEGF }\end{array}$ \\
\hline$C_{H}$ & 1 & 19 & $\begin{array}{l}\text { Concentration of } \\
\text { hypertrophic } \\
\text { chondrocytes }\end{array}$ \\
\hline$\chi_{V E G F}$ & 0.0001 & 19 & $\begin{array}{l}\text { Constant liberation of } \\
\text { VEGF by the } \\
\text { hypertrophic } \\
\text { chondrocytes }\end{array}$ \\
\hline
\end{tabular}




\begin{tabular}{|c|c|c|c|}
\hline$P_{V E G F}$ & 0.2 & 19 & $\begin{array}{l}\text { Quantity of VEGF } \\
\text { molecule widespread } \\
\text { in the domain }\end{array}$ \\
\hline$\alpha_{P T H r P}^{i}$ & 0.020 & 20 and 21 & $\begin{array}{l}\text { Constants that } \\
\text { indicate the influence }\end{array}$ \\
\hline$\beta_{i h h}^{i}$ & 0.020 & 20 & $\begin{array}{l}\text { of the concentration } \\
\text { of each molecule on } \\
\text { the isometric growth }\end{array}$ \\
\hline$\alpha_{\nabla P T H r P}^{i}$ & 0 & 20 and 21 & $\begin{array}{l}\text { Constants which } \\
\text { determine how much } \\
\text { the gradient of the }\end{array}$ \\
\hline$\beta_{\nabla i h h}^{i}$ & 0 & 20 & $\begin{array}{l}\text { concentration of each } \\
\text { molecule influences } \\
\text { on the directional } \\
\text { growth }\end{array}$ \\
\hline$d_{i}$ & 0 & 20 and 21 & $\begin{array}{l}\text { Constant related with } \\
\text { tissue elongation }\end{array}$ \\
\hline$\varphi_{H}^{D}$ & 10 & 21 & $\begin{array}{l}\text { Growth constants in } \\
\text { the dynamic state }\end{array}$ \\
\hline$S_{I h h}^{T h r}$ & 0.93 & 22 & $\begin{array}{c}\text { Threshold value of } \\
\text { the concentration of } \\
\text { Ihh in the } \\
\text { perichondrium }\end{array}$ \\
\hline$S_{P T H r P}^{T h r}$ & 0.4 & 23 & $\begin{array}{l}\text { Threshold value of } \\
\text { the concentration of } \\
\text { PTHrP in the } \\
\text { hypertrophic zone of } \\
\text { the growth plate }\end{array}$ \\
\hline$\mu_{x, y}$ & $\begin{array}{c}\text { For } \mathrm{x}=3 \\
\text { For } \mathrm{y}=15\end{array}$ & 23 & Growth constant \\
\hline
\end{tabular}


Appendix B: Endochondral ossification process for all cases studied in the computational model.
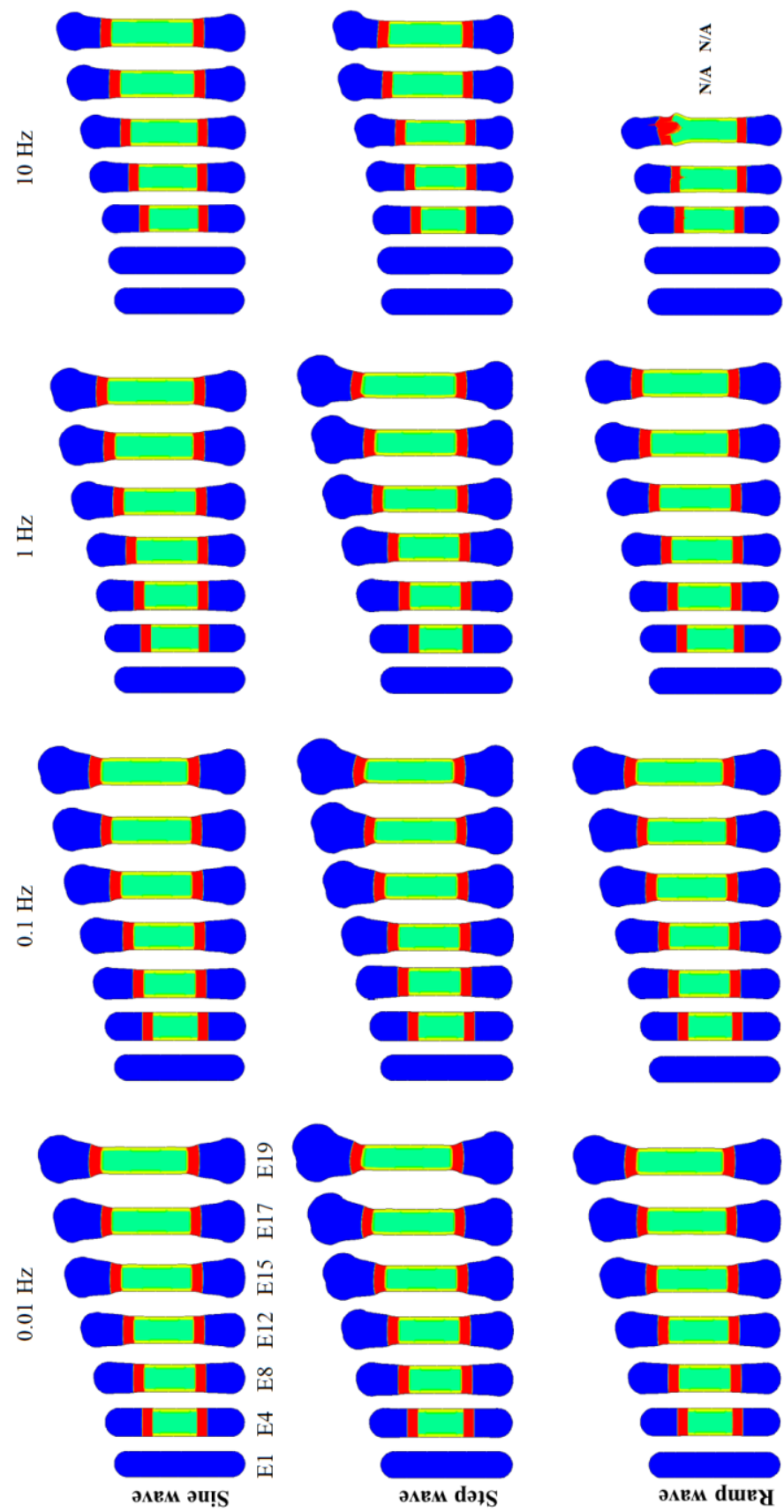


\section{Appendix C: Variation of the length and the thickness of the POC and distal and proximal epiphysis when the mechanical load was applied with different frequencies and signals.}

\begin{tabular}{|c|c|c|c|c|c|c|c|c|c|c|}
\hline \multirow{6}{*}{$\begin{array}{l}\text { Length of } \\
\text { the femur } \\
\qquad(\mathrm{mm})\end{array}$} & & \multicolumn{3}{|c|}{$\begin{array}{c}15 \text { days of } \\
\text { embryonic stage }\end{array}$} & \multicolumn{3}{|c|}{$\begin{array}{c}17 \text { days of } \\
\text { embryonic stage }\end{array}$} & \multicolumn{3}{|c|}{$\begin{array}{l}19 \text { days of embryonic } \\
\text { stage }\end{array}$} \\
\hline & $\begin{array}{l}\text { Frequency } \\
(\mathrm{Hz})\end{array}$ & Sine & Step & Ramp & Sine & Step & Ramp & Sine & Step & Ramp \\
\hline & 0.01 & 1.41 & 1.51 & 1.42 & 1.50 & 1.64 & 1.51 & 1.60 & 1.79 & 1.61 \\
\hline & 0.1 & 1.42 & 1.52 & 1.42 & 1.50 & 1.64 & 1.51 & 1.60 & 1.76 & 1.61 \\
\hline & 1 & 1.37 & 1.50 & 1.36 & 1.43 & 1.60 & 1.43 & 1.51 & 1.46 & 1.51 \\
\hline & 10 & 1.31 & 1.33 & 1.34 & 1.36 & 1.4 & N/A & 1.42 & 1.47 & N/A \\
\hline $\begin{array}{l}\text { Length of } \\
\text { the femur } \\
\text { without } \\
\text { load } \\
(\mathrm{mm})\end{array}$ & ---- & & 1.26 & & & 1.30 & & & 1.34 & \\
\hline \multirow{4}{*}{$\begin{array}{l}\text { Thickness } \\
\text { of the } \\
\text { distal } \\
\text { epiphysis } \\
\text { (mm) }\end{array}$} & 0.01 & 0.30 & 0.35 & 0.31 & 0.32 & 0.39 & 0.32 & 0.35 & 0.43 & 0.35 \\
\hline & 0.1 & 0.30 & 0.35 & 0.31 & 0.32 & 0.38 & 0.32 & 0.35 & 0.43 & 0.36 \\
\hline & 1 & 0.29 & 0.33 & 0.29 & 0.30 & 0.36 & 0.30 & 0.32 & 0.41 & 0.32 \\
\hline & 10 & 0.27 & 0.28 & 0.26 & 0.28 & 0.29 & N/A & 0.28 & 0.31 & N/A \\
\hline $\begin{array}{c}\text { Thickness } \\
\text { of the } \\
\text { distal } \\
\text { epiphysis } \\
\text { without } \\
\text { load } \\
(\mathrm{mm})\end{array}$ & ---- & & 0.23 & & & 0.24 & & & 0.24 & \\
\hline \multirow{4}{*}{$\begin{array}{l}\text { Thickness } \\
\text { of the } \\
\text { proximal } \\
\text { epiphysis } \\
(\mathrm{mm})\end{array}$} & 0.01 & 0.32 & 0.38 & 0.32 & 0.34 & 0.42 & 0.34 & 0.37 & 0.47 & 0.38 \\
\hline & 0.1 & 0.32 & 0.37 & 0.32 & 0.34 & 0.41 & 0.34 & 0.37 & 0.46 & 0.35 \\
\hline & 1 & 0.30 & 0.34 & 0.28 & 0.31 & 0.38 & 0.31 & 0.34 & 0.40 & 0.33 \\
\hline & 10 & 0.26 & 0.28 & 0.26 & 0.28 & 0.30 & N/A & 0.30 & 0.32 & N/A \\
\hline $\begin{array}{l}\text { Thickness } \\
\text { of the } \\
\text { proximal } \\
\text { epiphysis } \\
\text { without } \\
\text { load } \\
(\mathrm{mm})\end{array}$ & ---- & & 0.23 & & & 0.24 & & & 0.24 & \\
\hline
\end{tabular}


Appendix C

\begin{tabular}{|c|c|c|c|c|c|c|c|c|c|c|}
\hline \multirow{4}{*}{$\begin{array}{c}\text { Thickness } \\
\text { of the } \\
\text { POC } \\
(\mathrm{mm})\end{array}$} & 0.01 & 0.20 & 0.20 & 0.20 & 0.20 & 0.20 & 0.20 & 0.20 & 0.20 & 0.20 \\
\hline & 0.1 & 0.20 & 0.20 & 0.20 & 0.20 & 0.20 & 0.20 & 0.20 & 0.20 & 0.20 \\
\hline & 1 & 0.20 & 0.20 & 0.20 & 0.20 & 0.20 & 0.20 & 0.20 & 0.20 & 0.20 \\
\hline & 10 & 0.20 & 0.20 & 0.20 & 0.20 & 0.20 & N/A & 0.20 & 0.20 & N/A \\
\hline $\begin{array}{c}\text { Thickness } \\
\text { of the } \\
\text { POC } \\
\text { without } \\
\text { load } \\
(\mathrm{mm})\end{array}$ & ---- & & 0.2 & & & 0.2 & & & 0.2 & \\
\hline
\end{tabular}




\section{Appendix D: Molecular diffusion during bone growth.}

Ihh diffusion within the bone for all cases studied in the computational model.
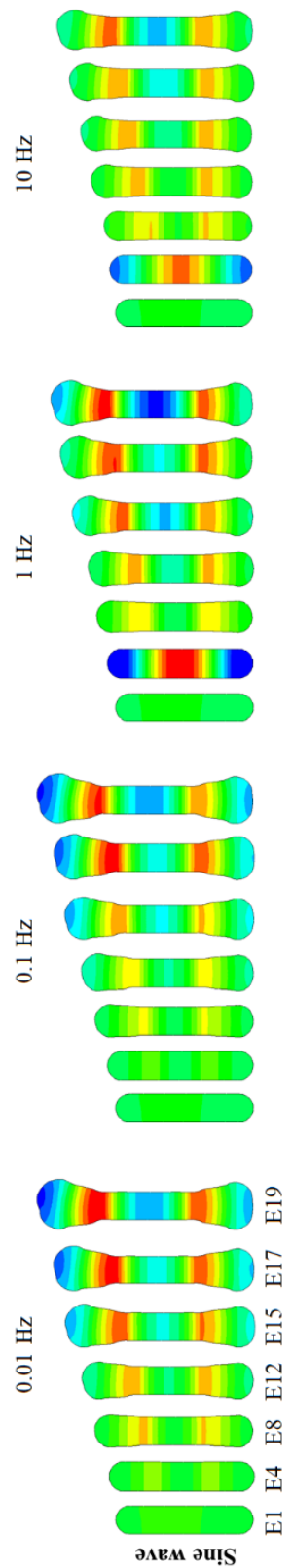
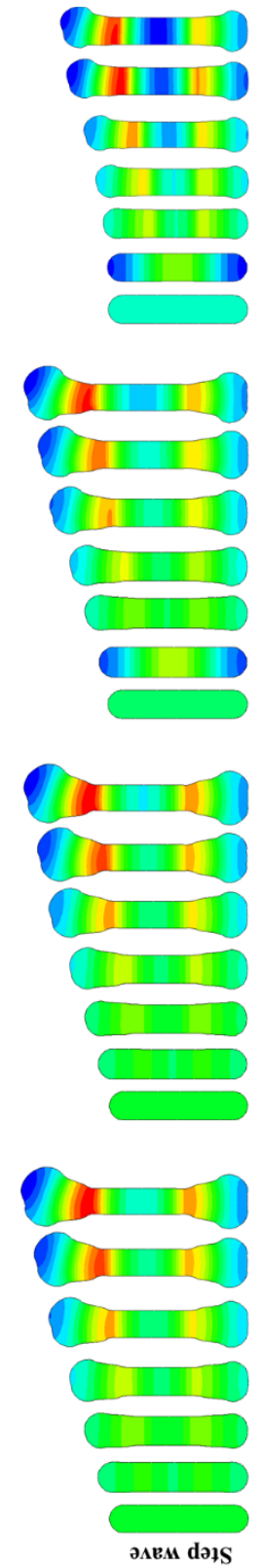
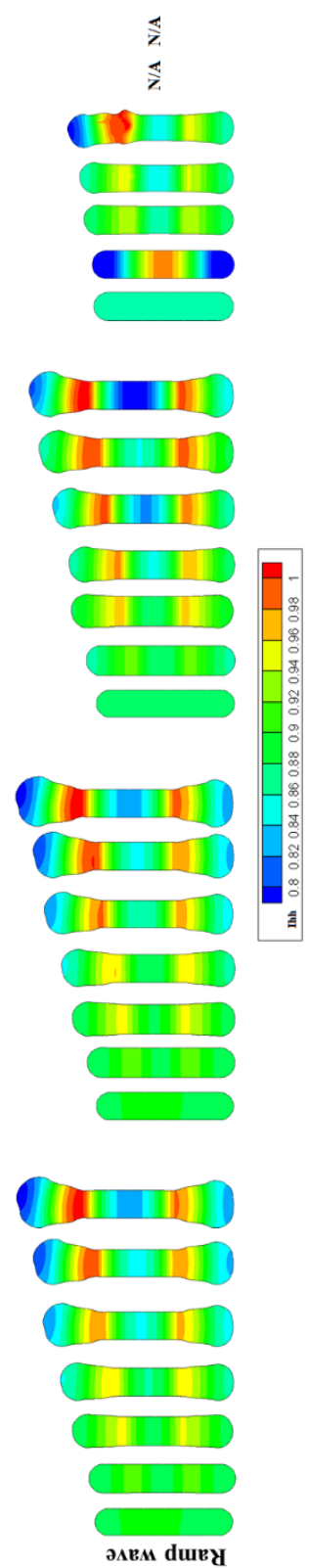
PTHrP diffusion within the bone for all cases studied in the computational model.
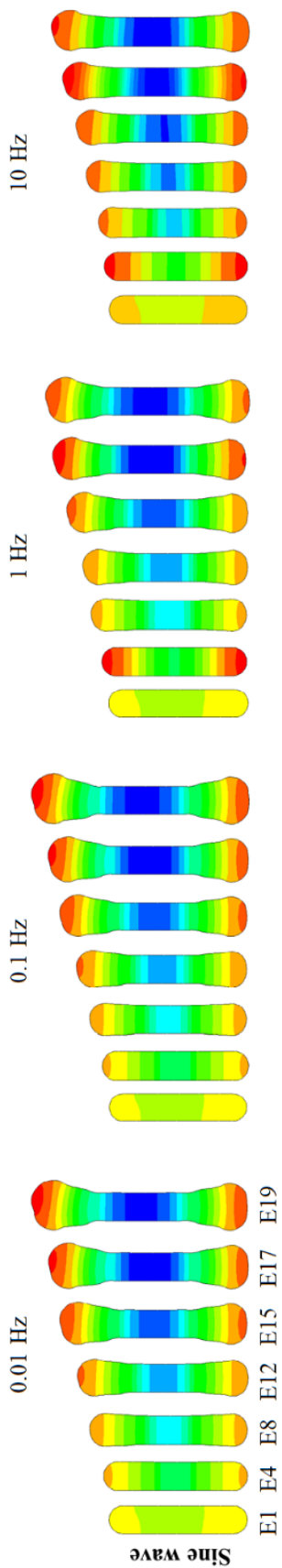
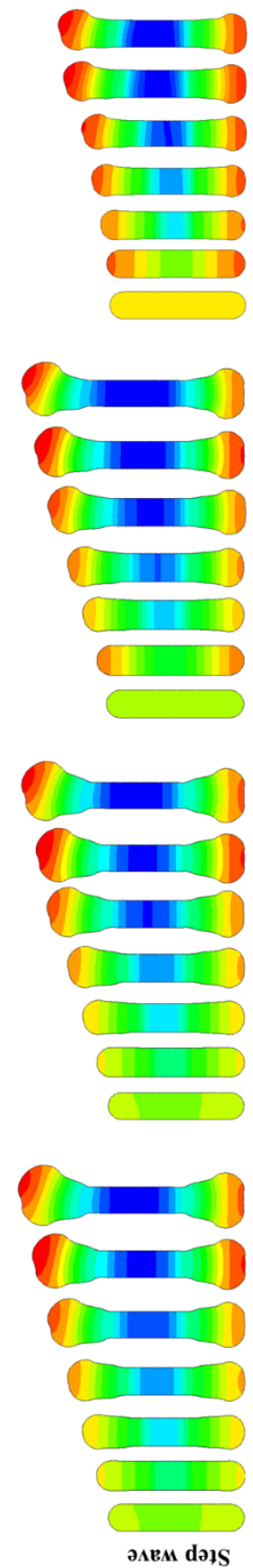
VEGF diffusion within the bone for all cases studied in the computational model.
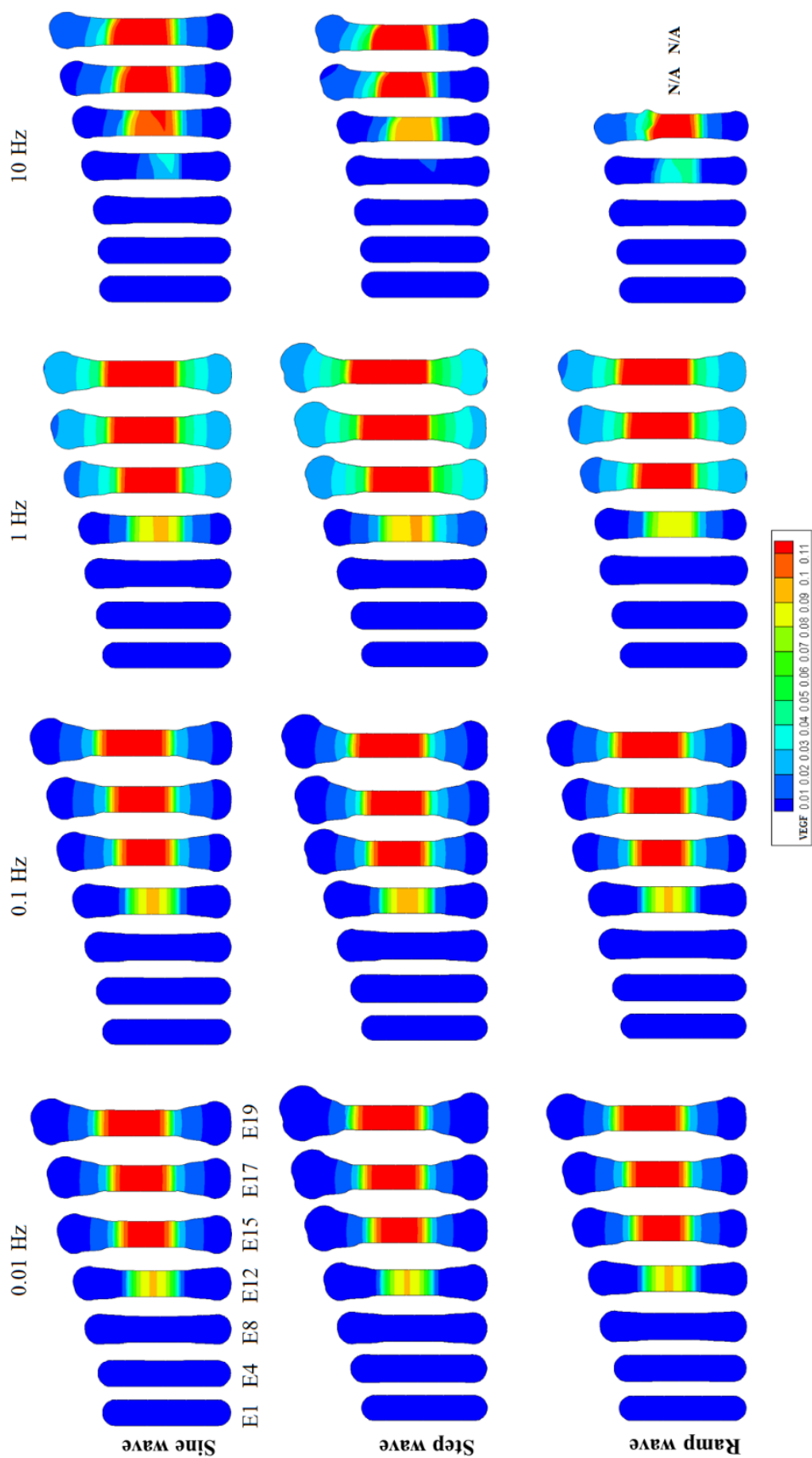
Appendix E: Bone morphology and Ihh, PTHrP and VEGF diffusion for simulations performed with high magnitude mechanical loading (1, 10 and $100 \mathrm{MPa})$.

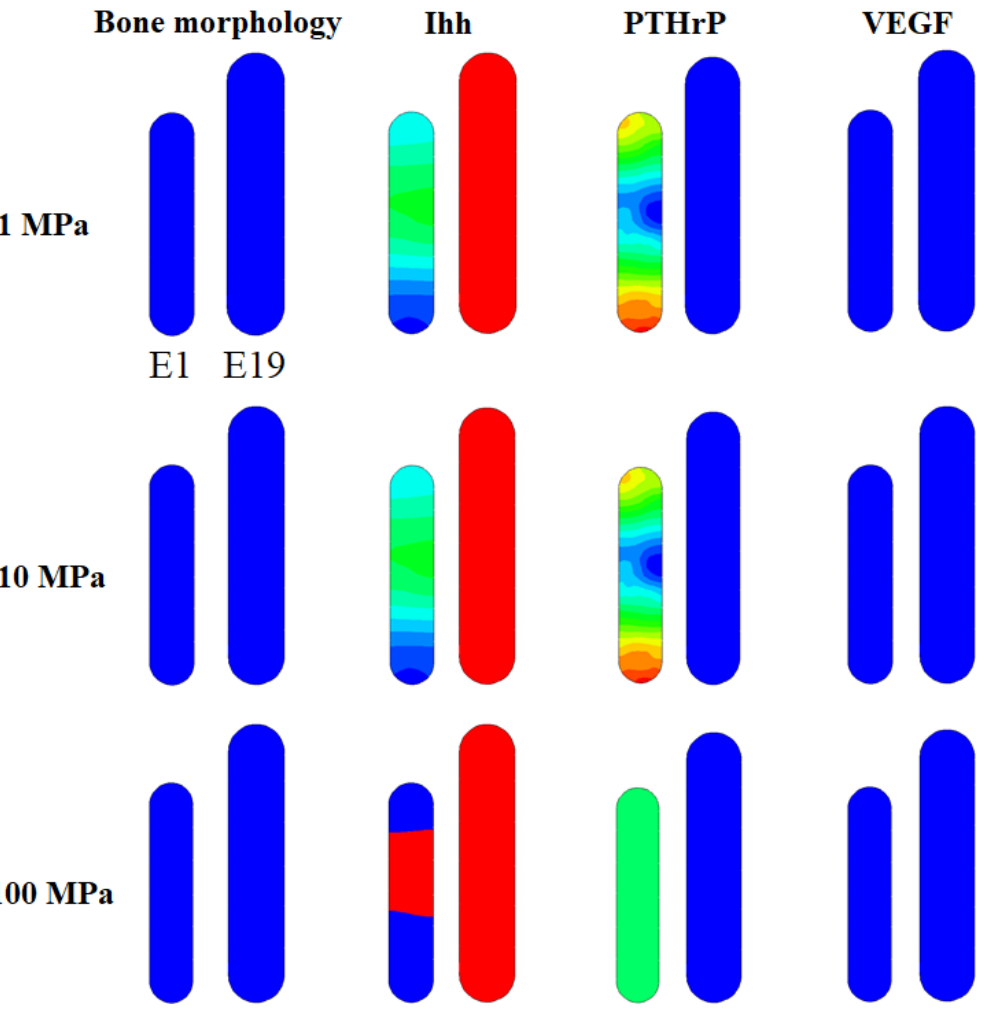




\section{Appendix F: Representative scheme of the EFs in the capacitively} coupling system generated by a voltage $50 \mathrm{Vpp}$ at $60 \mathrm{kHz}$ sine wave-

\section{form.}

A) EFs in an empty capacitive system and with bone explants and culture medium. B) Measurement points of the EFs within and outside the well-plate. C) On the left, the EF in the empty capacitive system is illustrated; on the right, the EF distribution in the capacitor with the explant bone and culture medium is shown.

$\mathbf{A}$

Freq / $60 \mathrm{kHz:} \mathrm{EF} \mathrm{in} \mathrm{an}$
empty well-plate $(\mathrm{mV} / \mathrm{cm})$

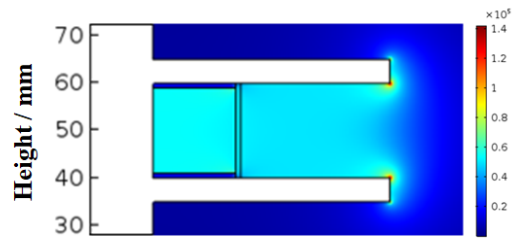

B

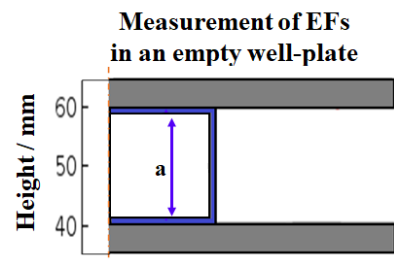

C

C Distribution of the EF in an

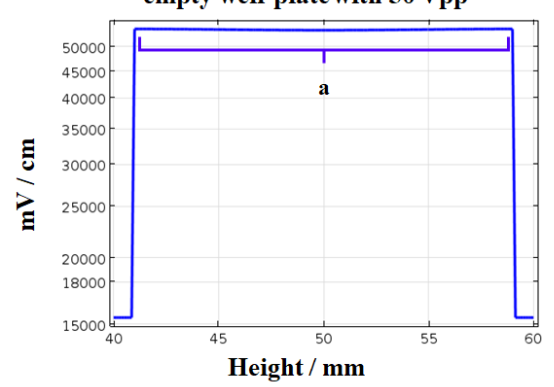

Freq / $60 \mathrm{kHz}$ : EF in a well-plate with bone explant and culture medium $(\mathrm{mV} / \mathrm{cm})$

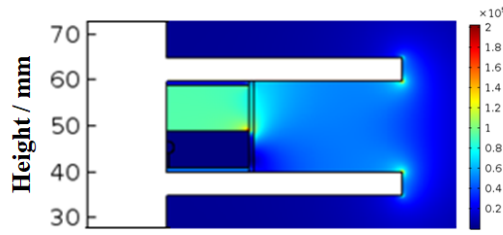

Measurement of EFs in well-plate with explants and culture medium
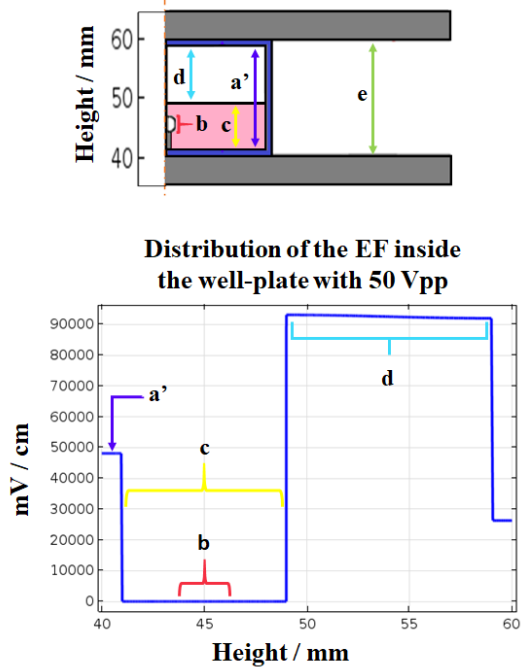
Appendix G: EF intensities within the capacitive coupled system applying a voltage $50 \mathrm{Vpp}$ at $60 \mathrm{kHz}$ sine wave-form.

A) EF within the chondroepiphysis. B) The EF in the culture medium.

C) The EF in the air gap between the culture medium and the top of the well-plate. D) The EF outside the well-plate between the electrodes.

A Distribution of the EF inside the chondroepiphysis with $50 \mathrm{Vpp}$

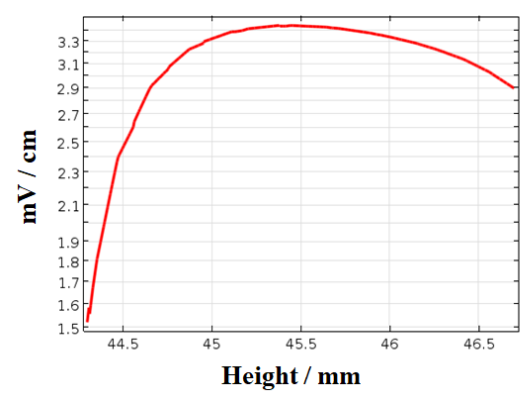

Distribution of the EF in the air
inside the well-plate with $50 \mathrm{Vpp}$

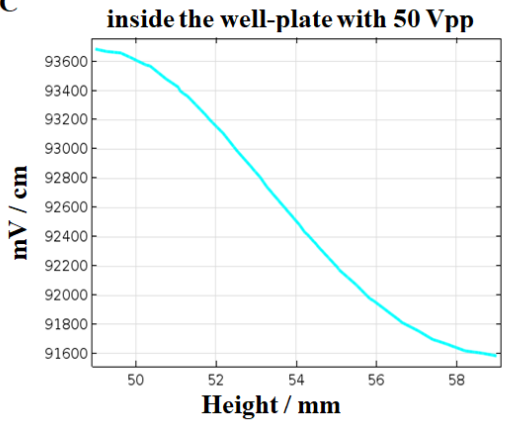

Distribution of the EF in the
culture medium with $50 \mathrm{Vpp}$

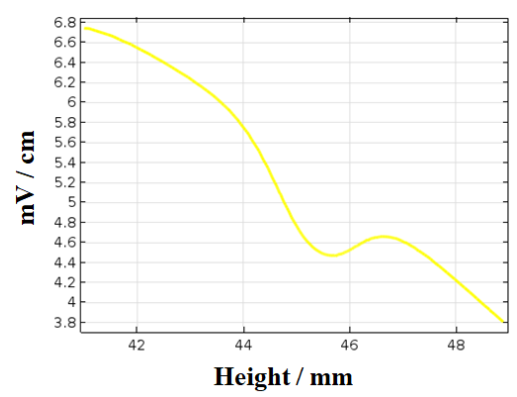

Distribution of the EF outside
the well-plate with 50 Vpp 
Appendix H: Measurement of growth plate lengths and thickness of $\boldsymbol{P}-\mathrm{z}, \mathrm{PH}-\mathrm{z}$ and $\mathrm{Ht}-\mathrm{z}$.

A) Length of proximal humerus growth plates. B) Thickness of proximal humerus epiphyseal plate zones. Statistically significant differences were found between controls and stimulated explants in term of length and thickness after a stimulation with EFs of 3.5 and

$7.5 \mathrm{mV} / \mathrm{cm}(p<0,05)$, except those marked with " $n s$ " (not significant).

$\mathbf{A}$

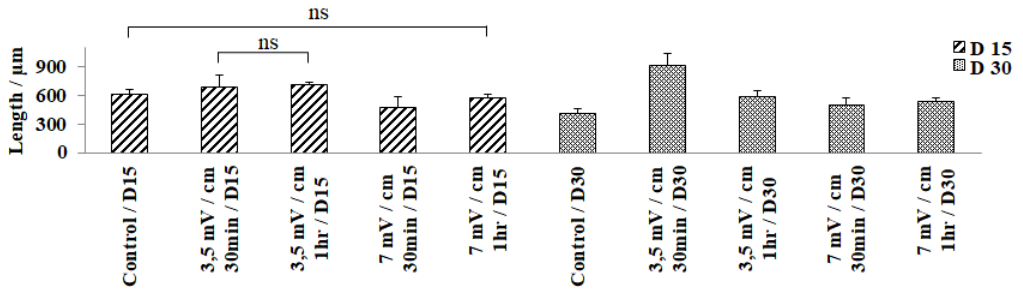

$\mathbf{B}$

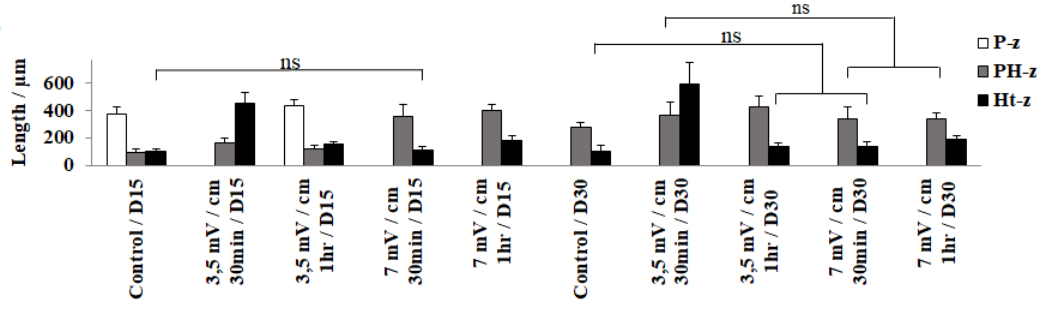


Appendix I: Measurement of $C_{i}$ and $C_{c}$ in growth plate zones of humerus explants.

A) Graphic bars of $\boldsymbol{C}_{\boldsymbol{i}}$ within growth plate zones of humerus. B) Graphic bars of $\boldsymbol{C}_{\boldsymbol{c}}$ within growth plate zones of humerus. Statistically significant differences were found between controls and stimulated explants after a stimulation with EFs of 3.5 and $7.5 \mathrm{mV} / \mathrm{cm}(p<$ $\left.0,05^{* *}\right)$, except those marked with " $n s$ " (not significant).

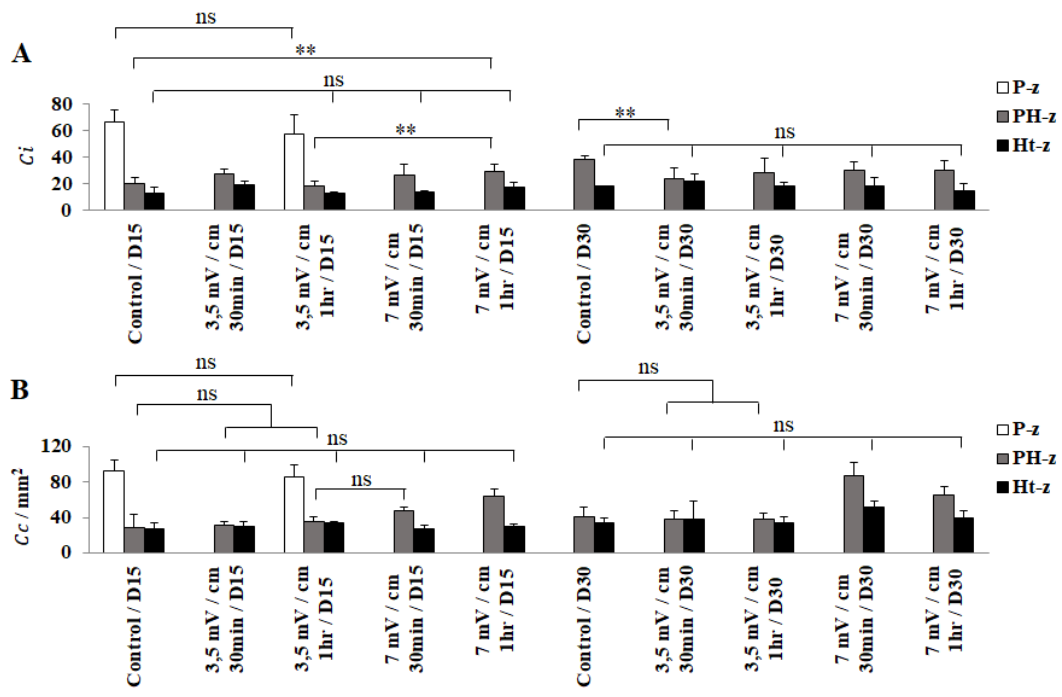


Appendix J: Measurement of $C_{D}$ and inclination angle $\alpha$ of columns in growth plate zones of humerus explants.

A) Graphic bars of $\boldsymbol{C}_{\boldsymbol{D}}$ within growth plate zones of humerus. B) Graphic bars of $\alpha$ within growth plate zones of humerus. Statistically significant differences were found between controls and stimulated explants after a stimulation with EFs of 3.5 and $7.5 \mathrm{mV} / \mathrm{cm}(p<$ $\left.0,05^{* *}\right)$, except those marked with "ns" (not significant).

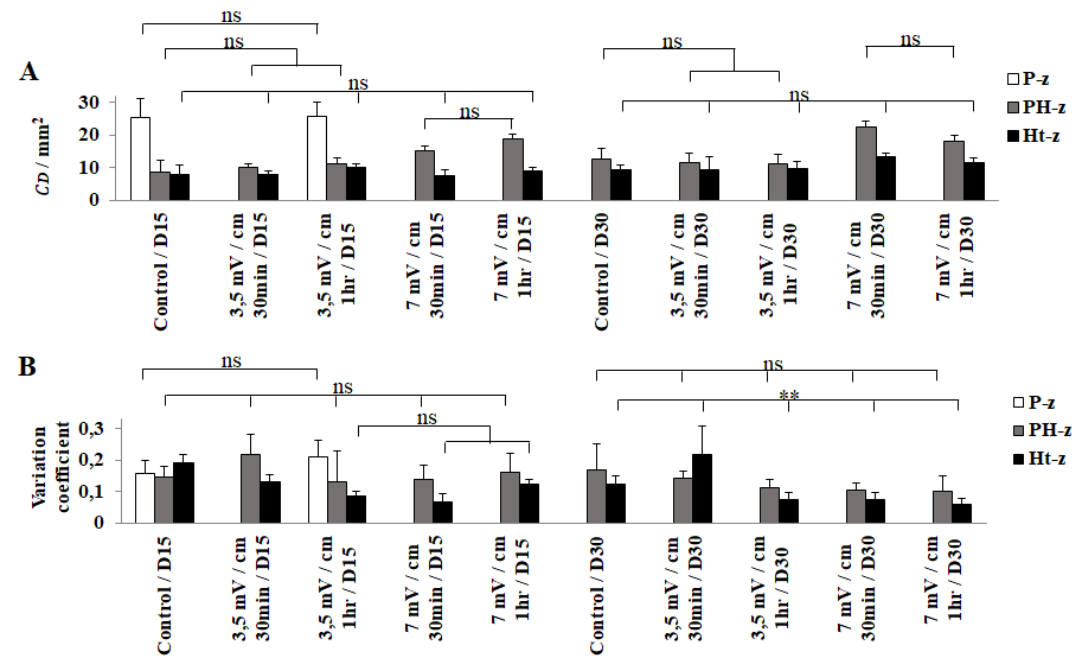

